\title{
IMMUNE MEDIATORS OF MURINE LYME ARTHRITIS
}

\author{
A Dissertation \\ Presented to \\ The Faculty of the Graduate School \\ At the University of Missouri \\ In Partial Fulfillment \\ of the Requirements for the Degree
}

Doctor of Philosophy

By

Anna Maria Cunningham

Dr. Charles R. Brown, Dissertation Advisor

May 2014 
The undersigned, appointed by the dean of the Graduate School, have examined the dissertation entitled

\section{IMMUNE MEDIATORS OF MURINE LYME ARTHRITIS}

Presented by Anna M. Cunningham,

A candidate for the degree of doctor of philosophy

And hereby certify that, in their opinion, it is worthy of acceptance

Professor Charles R. Brown

Professor Mark A. Daniels

Professor Daniel E. Hassett

Professor Kevin L. Fritsche

Professor Susan C. McKarns 


\section{DEDICATION}

I dedicate this dissertation to my family: My parents, Forrest (Mike) and Paula Ritzman whom taught me the value of hard work both verbally and visually by serving as living examples; my sister Maggie whose sass kept me smiling through everything; and my husband Cody whose belief in me I will never understand.

Mom and dad, I have never known more hardworking individuals as you two. Your support through my academic and personal struggles has always been unfaltering and I have never once doubted that I have an amazing support system.

Maggie, thank you for being my best friend and most trusted confidant. Whenever life gets tough, I turn to you for your wisdom and your advice. No one can calm me down from a "freak out" better than you. Thank you for helping me to calm down- one of the most important lessons I have learned in this life.

Cody, thank you for choosing me to spend your spectacular life with. I know you hate clichés, but you make life worth living. Your intelligence, sense of humor, incredible athleticism, and calm demeanor make every day an adventure. Thank you for putting up with me.

Mom, Dad, Maggie, and Cody: thank you for believing in me when I didn't believe in myself. Thank you for picking me up when I fell down. Thank you for encouraging me when the future seemed bleak. Thank you for teaching me how to be a good person and to never sacrifice kindness or compassion in an effort to get ahead. You are all my heart and I love you more than anything. 


\section{ACKNOWLEDGMENTS}

I would like to acknowledge my incredible advisor Dr. Charles R. Brown. As a student, I was difficult at times due to my inability to decide a future direction and my inability to settle on a project. However, Dr. Brown allowed me to make mistakes and learn from them and also allowed me to explore all of my options. He supported and encouraged me through my entire graduate school career and helped me grow both academically and personally. Although research was difficult, Dr. Brown kept it light with a wonderful sense of humor, for which I am grateful. I could not have chosen a better advisor.

I would like to acknowledge my committee, Drs. Daniels, Hassett, Fritsche, and McKarns, who supported me and encouraged me along the way.

I would also like to acknowledge the faculty of Missouri Southern State University, especially Drs. James Jackson and Melvyn Mosher. I received an outstanding education at MSSU and was wholeheartedly supported and encouraged by my wonderful advisors. I would not be where I am today without their help and guidance and I am eternally grateful for their support and friendship.

Finally, I would like to acknowledge my family. Graduate school required the majority of my focus and led to my absence or distraction during many important events. For their understanding and continuing support and love, I am eternally grateful. I love you.

To everyone that supported me and encouraged me along the way: Thank you. This Ph. D. is just as much yours as mine. 


\section{TABLE OF CONTENTS}

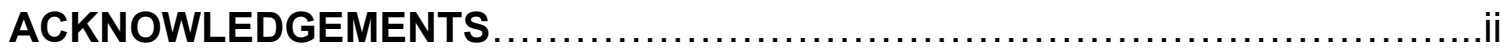

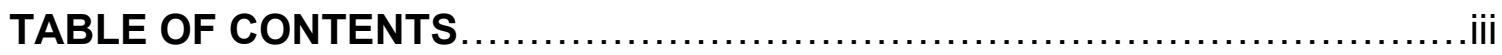

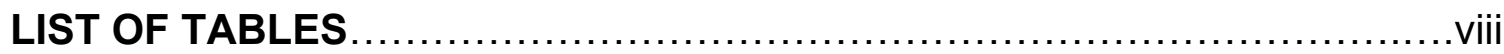

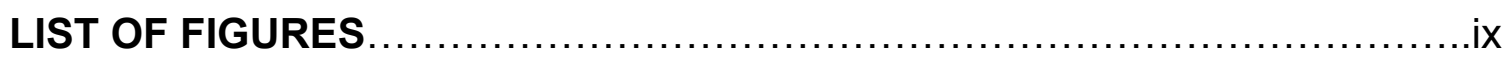

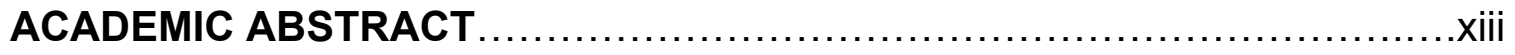

IMMUNE MEDIATORS OF MURINE LYME ARTHRITIS

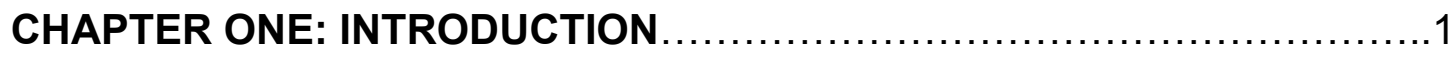

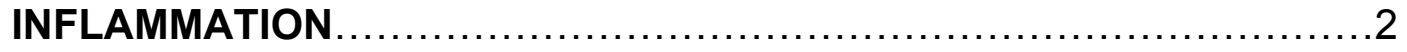

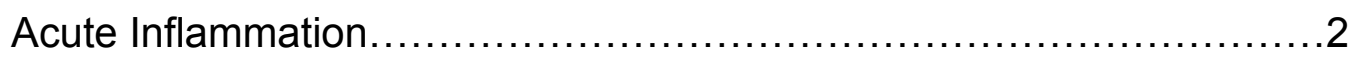

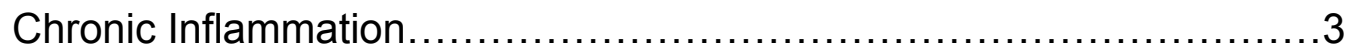

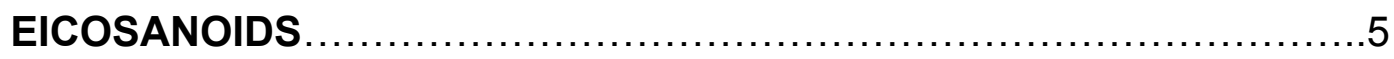

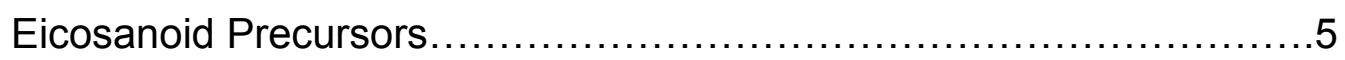

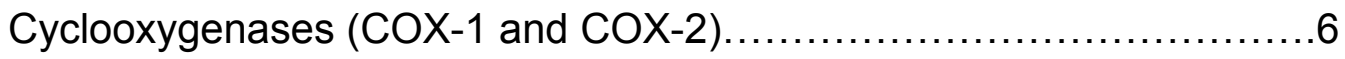

Lipoxygenases $(5-$ LOX and 12/15-LOX) .............................

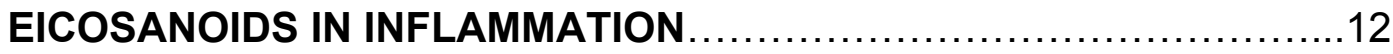

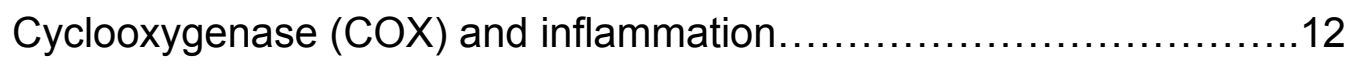

Lipoxygenase $($ LOX) and inflammation............................ 18

LYME ARTHRITIS AS A MODEL OF INFLAMMATION $\ldots \ldots \ldots \ldots \ldots \ldots \ldots 21$

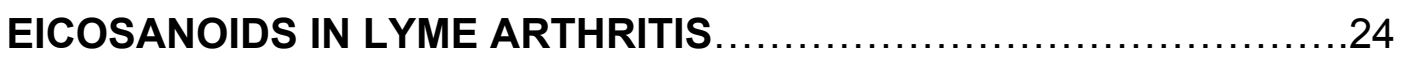

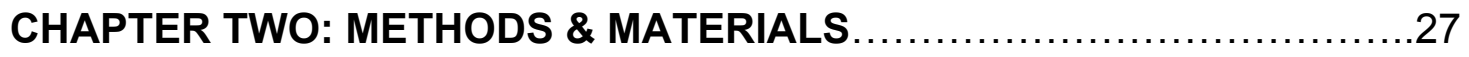

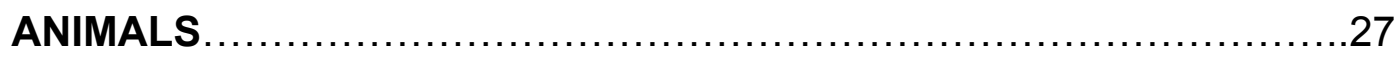

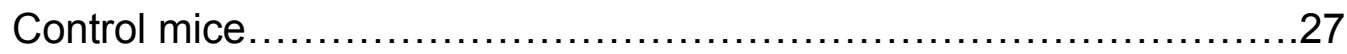


$\mathrm{KC}^{-/-}$mice.

KRN, NOD, and K/BxN mice.......................................27

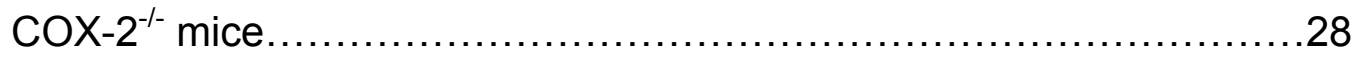

Housing and experimental conditions ...............................28

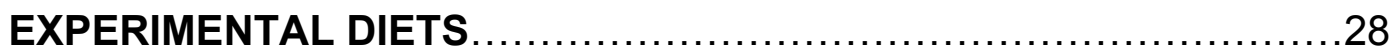

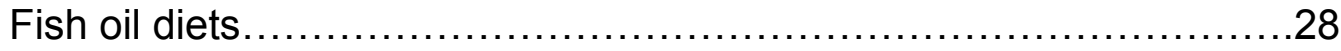

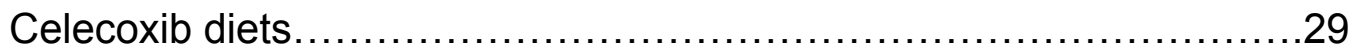

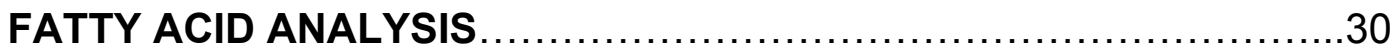

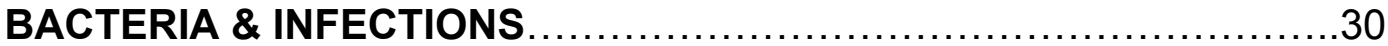

ASSESSMENT OF JOINT SWELLING .................................31

ASSESSMENT OF TISSUE PATHOLOGY \& IMMUNOCHEMISTRY ......32 EXTRACTION OF DNA FROM TISSUES USING TRIZOL ...............33 QUANTIFICATION OF BORRELIA BURGDORFERI IN TISSUES.........34 QUANTIFICATION OF TISSUE CYTOKINE \& CHEMOKINE

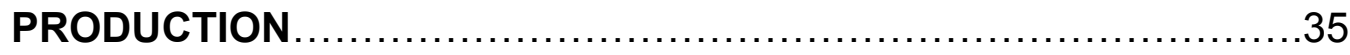
EXTRACTION OF TISSUE EICOSANOIDS ..............................36 LC, MS, \& EICOSANOID QUANTIFICATION ..........................37

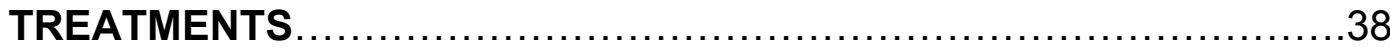

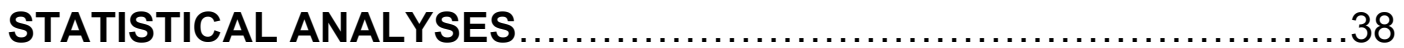

CHAPTER THREE: KC (CXCL1) MEDIATES THE DEVELOPMENT OF

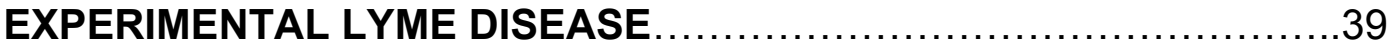

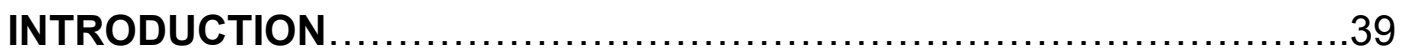

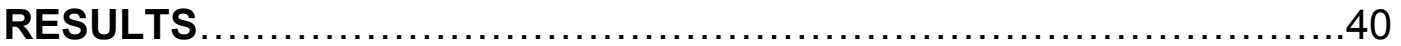


KC mediates edema resulting from infection with Borrelia burgdorferi.40

$\mathrm{KC}$ mediates Lyme arthritis severity.

Absence of $\mathrm{KC}$ leads to reduced neutrophil and macrophage joint infiltration .44

Absence of KC does not affect the ability of spirochetes to be cleared from joints of $B b$-infected mice. 46

Joint cytokine and chemokine expression is unaffected by the absence of $\mathrm{KC}$. .48

KC mediates Lyme carditis severity. .50

Neutrophil and macrophage infiltration into heart tissue is altered in the absence of KC. .51

$\mathrm{Bb}$ clearance from heart tissue is intact in the absence of $\mathrm{KC}$ .54

Heart cytokine and chemokine production s unaffected by the absence of $\mathrm{KC}$ .54

DISCUSSION. .58

CHAPTER FOUR: THE EFFECT OF CYCLOOXYGENASE-2 DEFICIENCY ON THE CYTOKINE/CHEMOKINE/LIPID MILIEU DURING LYME ARTHRITIS 63 INTRODUCTION 63

RESULTS. .64

Inhibition of COX-2 results in attenuated ankle joint swelling, but has little effect on arthritis severity...... 64 Inhibition of COX-2 affects the production of numerous eicosanoids...67 
COX-2 metabolites are reduced in mice fed Celecoxib-containing chow.

Lipoxygenase (LOX) metabolites are differentially affected by COX-2 inhibition. .70

Supplementation of $\mathrm{COX}_{-} 2^{--}$mice with $\mathrm{PGJ}_{2}$ does not restore the ability to resolve arthritis. .73

Supplementation of $\mathrm{COX}_{-2} 2^{--}$mice with $\mathrm{PGE}_{2}$ does not restore the ability to resolve arthritis .76

Cytokine and chemokine production is reduced in $\mathrm{COX}-2^{-/-}$mice. .79

DISCUSSION .83

CHAPTER FIVE: THE EFFECT OF DIETARY FISH OIL SUBSTITUTION ON

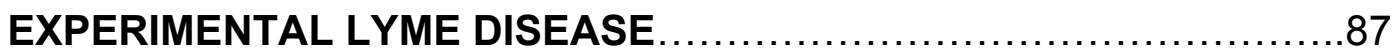

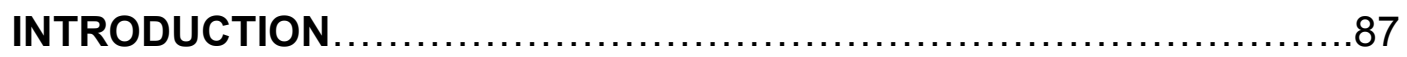

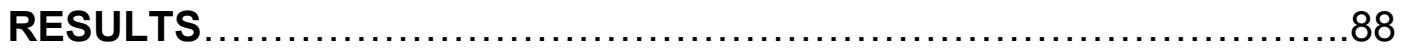

Dietary fish oil substitution alters the serum and liver fatty acid profile. 88 Dietary fish oil substitution alters global eicosanoid production.........91 Mono-hydroxylated fatty acid metabolites are produced primarily nonenzymatically irrespective of diet..............................96

Dietary fish oil substitution alters COX metabolite production..........101

Dietary fish oil substitution does not affect Lyme arthritis severity, joint immune cell composition, or joint $B b$ loads. 107

Dietary fish oil substitution does not affect Lyme carditis severity, heart immune cell composition, or cardiac $B b$ loads. 110 
DISCUSSION.

\section{CHAPTER SIX: DYNAMIC EICOSANOID PRODUCTION DURING} AUTOANTIBODY-DRIVE K/BXN SERUM-TRANSFER ARTHRITIS....118 INTRODUCTION ..................................................... 118

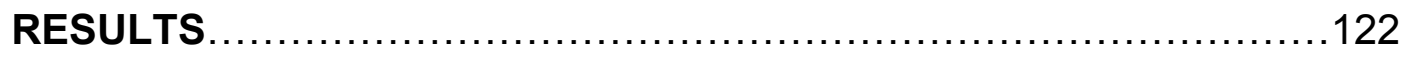

Passage of $\mathrm{K} / \mathrm{BxN}$ serum to $\mathrm{C} 3 \mathrm{H} / \mathrm{HeJ}$ mice results in severe polyarthritis................................................... 122

Eicosanoid enzymatic pathways are activated during KSTA..........124 Dynamic regulation of Cytochrome P450 metabolites during KSTA...128 Expression of non-enzymatically generated eicosanoids during KSTA .........................................................

Cyclooxygenase metabolite production during KSTA .................134

Lipoxygenase production during KSTA ...........................138

KSTA has a higher pro-inflammatory index than Lyme arthritis........140

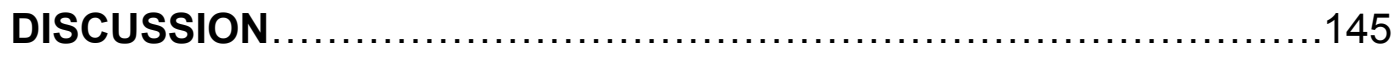

CHAPTER SEVEN: CONCLUSIONS ......................................150

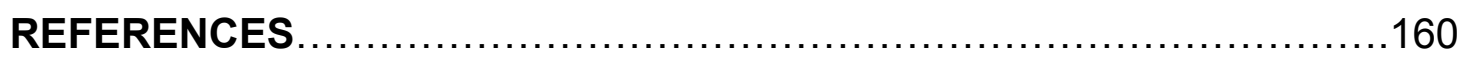

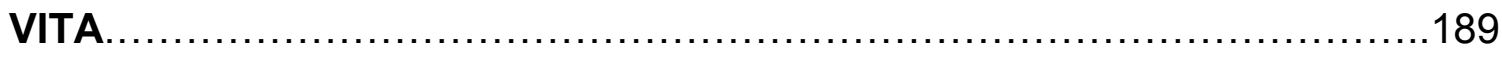




\section{LIST OF TABLES}

\section{CHAPTER FIVE}

5.1. Dietary fish oil substitution alters the serum and liver fatty acid profile......90

5.2. Dietary fish oil substitution results in a global shift in eicosanoid

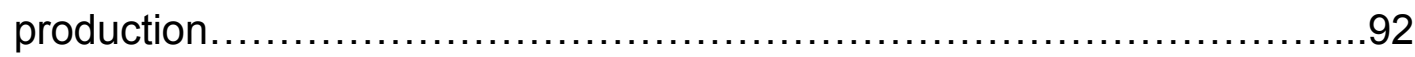

5.3. EPA- and DHA-derived MHM are increased in fish oil-fed mice.............100 


\section{LIST OF FIGURES}

\section{CHAPTER ONE}

1.1. The major metabolic pathways of eicosanoid generation.................

1.2. Cyclooxygenase enzymes metabolize arachidonic acid into

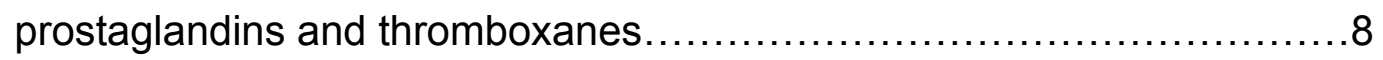

1.3. Lipoxygenase enzymes metabolize AA into leukotrienes.................10

1.4. COX-2 has a bimodal expression pattern during an inflammatory

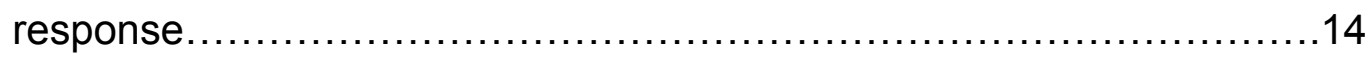

\section{CHAPTER THREE}

3.1. $\mathrm{KC}^{-/-}$mice have reduced joint edema in response to infection with Borrelia burgdorferi. .42

3.2. $\mathrm{KC}^{-/-}$mice exhibit attenuated arthritis severity and altered joint cell infiltrate.

3.3. $\mathrm{KC}^{-/-}$mice have significantly reduced neutrophil and macrophage infiltration into infected joint tissue. .45

3.4. $\mathrm{KC}^{-/}$mice are able to clear $\mathrm{Bb}$ infection as well as WT mice. 47

3.5. $\mathrm{KC}^{-/-}$mice are able to effectively produce cytokines and chemokines to WT levels .49

3.6. $\mathrm{KC}^{-/-}$mice have attenuated Lyme carditis and altered immune cell infiltration into $B b$-infected heart tissue. 52

3.7. $\mathrm{KC}^{-/-}$mice have significantly reduced numbers of neutrophils and macrophages in $B b$-infected heart tissue 
3.8. $\mathrm{KC}^{-/-}$mice are not defective in their ability to clear $\mathrm{Bb}$ infection from cardiac tissue .55

3.9. Cytokine and chemokine levels in heart tissue of $\mathrm{KC}$ mice are unaltered.

\section{CHAPTER FOUR}

4.1. Progression of arthritis in Celecoxib-fed mice............................66

4.2. Global eicosanoid production in Celecoxib-fed mice.....................68

4.3. Production of cyclooxygenase-derived metabolites in the ankle joints of Celecoxib-fed mice.

71

4.4. Production of lipoxygenase metabolites in the ankle joints of Celecoxibfed mice. .72

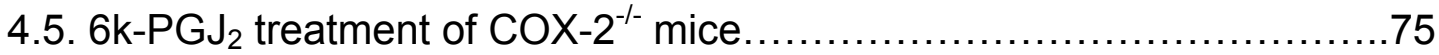

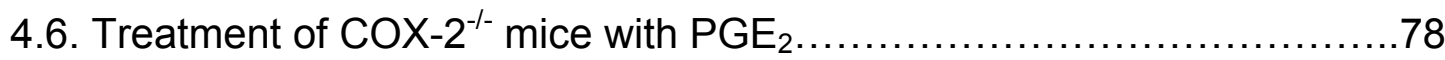

4.7. Treatment of COX $-2^{-/-}$mice with the EP2 agonist Butaprost.............80

4.8. Cytokine and chemokine production in the ankle joints of Celecoxib-fed mice.

4.9. Cytokine and chemokine production in $\mathrm{COX}-2^{-/-}$mice 84

\section{CHAPTER FIVE}

5.1. Eicosanoids regulate all phases of Lyme arthritis 89

5.2. Dietary fish oil substitution results in a global shift in eicosanoid generation

5.3. Dietary fish oil substitution results in increased levels of EPA- and DHAderived mono-hydroxylated metabolites (MHM). 97 
5.4. The majority of mono-hydroxylated metabolites produced in fish oil-fed mice are generated non-enzymatically.....

5.5. Cyclooxygenase-derived AA metabolites are reduced in fish oil diet-fed mice. 103

5.6. Dietary fish oil substitution results in the preferential use of EPA and DHA substrates for metabolism via cyclooxygenase-2

5.7. Dietary fish oil substitution results in a redirection of COX-2-mediated AA

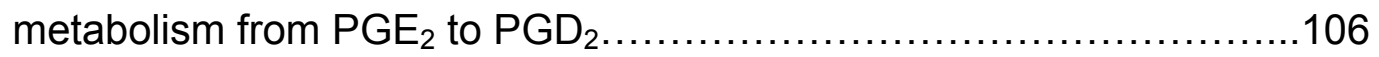

5.8. Dietary fish oil does not alter the progression of Lyme arthritis..........108

5.9. Dietary fish oil substitution does not alter progression of Lyme carditis 111

\section{CHAPTER SIX}

6.1. Severe arthritis develops in response to passage of $\mathrm{K} / \mathrm{BxN}$ serum to $\mathrm{C} 3 \mathrm{H} / \mathrm{HeJ}$ mice 123

6.2. Joint eicosanoids are dynamically affected by passage of $\mathrm{K} / \mathrm{BxN}$ serum. 125

6.3. Eicosanoid enzymatic pathways engaged during KSTA 127

6.4. Cytochrome P450-derived hydroxyeicosatetraenoic acids (HETEs) expression in joints of arthritic mice.

6.5. Cytochrome P450-derived epoxyeicosatetraenoic acids (EETs) are produced in the joints of arthritic mice. .131

6.6. Non-enzymatic eicosanoid production in joints of arthritic mice. 133

6.7. Cyclooxygenase (COX)-derived metabolite production in the joints.....135 
6.8. 5-Lipoxygenase (5-LOX)-derived metabolite production in the joints of arthritic mice............................................................ 139

6.9. 12/15-lipoxygenase (12/15-LOX)-derived metabolite production in the

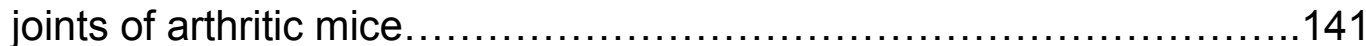

6.10. Inflammatory indices of mice induced to have either KSTA or Lyme

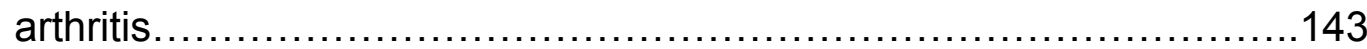




\section{ACADEMIC ABSTRACT}

Lyme disease is the most common vector-borne disease in both the United States and Europe; however, its pathogenesis is incompletely understood. The studies described in this thesis aid in the elucidation of mechanisms regulating murine Lyme arthritis and may suggest mechanisms by which human Lyme disease is mediated. (1) We found that the chemokine KC is responsible for neutrophil recruitment and subsequent development of Lyme arthritis and carditis. This was the first study to describe an immunological regulatory mechanism mediating disease susceptibility to murine Lyme disease, as resistant mice (B6) produce less $\mathrm{KC}$ than susceptible $(\mathrm{C} 3 \mathrm{H} / \mathrm{HeJ})$ mice and do not develop disease. (2) We found that metabolites produced via the COX-2 pathway are important for functional resolution and that resolution is likely mediated via the $\mathrm{PGE}_{2} / \mathrm{EP} 2$ axis. (3) We found that although dietary fish oil substitution leads to a

global shift in eicosanoid production (from AA/LA-derived eicosanoids to EPA/DHA-derived eicosanoids) and promotes anti-inflammatory prostaglandin production, disease severity is not altered. (4) We found that eicosanoid production throughout the course of autoantibody-drive K/BxN serum-transfer arthritis differs significantly from that seen during Lyme arthritis and that patterns of eicosanoid expression reflect the severity and kinetics of each type of arthritis. These studies aid in understanding the immunological mechanisms regulating the occurrence and severity of murine Lyme arthritis. 


\section{CHAPTER ONE}

\section{INTRODUCTION}

"...for I do believe that everyone should know the beautiful deeds of which his or her tissues are capable (1)." -Guido Majno, The Healing Hand

\section{INFLAMMATION}

\section{A. Acute Inflammation}

Classically, inflammation is defined as a controlled process by which immune mediators are recruited to a site of infection or tissue injury $(1,2)$. Stimulation of pattern recognition receptors (PRR), such as toll-like receptors (TLR) and nucleotide-binding oligomerization domain (NOD)-like receptors (NLR), by insult or injury leads to the activation of endothelial cells and the subsequent release of mediators that recruit leukocytes and vasoactive molecules that dilate blood vessels $(3,4)$. Leukocytes (mainly neutrophils) exit dilated blood vessels and are drawn to the site of infection or injury by chemokines (e.g.: $\mathrm{KC}$ ) and bioactive lipids (e.g.: leukotriene $\mathrm{B}_{4}, \mathrm{LTB}_{4}$ ) produced by the activated endothelium. In an effort to clear infection, neutrophils release an array of bioactive molecules [including reactive oxygen species (ROS), reactive nitrogen species (RNS), and cytokines] and phagocytose any microbial invaders.

Neutrophils also produce chemokines that recruit monocytes and macrophages $(5,6)$ to further clear infectious agents as well as remove any dead 
cells (including neutrophils that have undergone apoptosis). These macrophages and monocytes also promote tissue remodeling via the production of anti-inflammatory, pro-resolving cytokines, such as transforming growth factor beta (TGF- $\beta$ ), and lipids such as lipoxins (e.g.: $\mathrm{LXA}_{4}$ ), resolvins (e.g.: RvE 1 ), and protectins (e.g.: PD1) (7).

\section{B. Chronic Inflammation}

Inflammation that does not lead to the production of molecules that function to induce resolution can become chronic and harmful. Chronic inflammation leads to the continuous production of pro-inflammatory molecules, recruitment of leukocytes, release of pro-inflammatory granules, and the inability of tissues to heal and have their functions properly restored. The majority of studies chronicling classical pathways of inflammation have been conducted using models of infection (8). This is, for the most part, due to the relatively straightforward process of inflammation during an infection in which a trigger (pathogen) stimulates inflammation, which leads to the clearance of the trigger.

Once the trigger has been eliminated in these models, inflammation is no longer provoked and tissue remodeling can occur. However, inflammation in the pathological sense (chronic inflammation) is less understood due to the fact that often times no "trigger" has been identified.

Using infectious or transient models of inflammation, however, antiinflammatory/pro-resolving molecules have been identified that play active roles in halting inflammation and promoting tissue restoration and remodeling (e.g.: 
lipoxins, resolvins, and protectins) $(7,9-11)$. The discovery of these molecules and their anti-inflammatory/pro-resolving functions has led to an increased understanding of the end processes of inflammatory responses. Whereas once it was believed that the cessation of inflammation (resolution) was due to the elimination of a "trigger," now it is understood that resolution is an active process that not only requires elimination of a "trigger" (if one exists), but also production of molecules that inhibit pro-inflammatory pathways as well as directly promoting tissue healing.

In light of this information, it is now understood that chronic inflammation is due to a combination of dysregulated pro-inflammatory and pro-resolution machinery. Thus, a paradigm shift in treatment modalities for chronic inflammatory disease has occurred. Whereas, historically, anti-inflammatory agents such as anti-tumor necrosis factor (anti-TNF) have been recommended for treatment of chronic inflammatory disease (e.g.: rheumatoid arthritis), newer modalities focus on inducing pro-resolving pathways. The importance of this paradigm shift is the benefits that arise from not inhibiting normal, regulated inflammation. While chronic inflammation is detrimental, regulated inflammation is a very important means of restoring homeostasis to tissues that have encountered insult or injury. Side effects of anti-inflammatory agents, such as anti-TNF, include increased risk of infection. Treatments that target pro-resolving pathways would enable normal, regulated inflammation to take place, but would put a halt to dysregulated inflammatory responses that have become chronic and are causing more harm than good to the patient. 
Although increased understanding of pro-resolving pathways has identified a new target for the investigation of chronic inflammatory disease, these pathways are still relatively poorly understood due to the following: 1) many pro-resolving pathways are dependent on functional pro-inflammatory pathways (7); 2) many molecules have both pro-inflammatory and pro-resolving functions which are concentration, time, or even tissue-dependent (12); and 3) enzymatic pathways which produce pro-resolution metabolites also produce proinflammatory metabolites (13). Therefore, an increased understanding of these pathways will yield important information regarding therapeutic targets for the treatment of chronic inflammatory disease.

\section{EICOSANOIDS}

\section{A. Eicosanoid Precursors}

Eicosanoids are small lipids that contain 20 carbons and are derived from the omega-6 ( $\omega-6)$ fatty acids arachidonic acid (AA) and linoleic acid (LA) and the $\omega-3$ fatty acids eicosapentaenoic acid (EPA) and docosahexaenoic acid (DHA). AA, LA, EPA, and DHA are dietary fatty acids stored in the phospholipid bilayer of the plasma and nuclear membranes $(14,15)$. AA and LA are $20-25$-fold more prevalent than EPA and DHA in the cell membranes of humans consuming a "Western diet," as they are found in large amounts in soy, corn, beef, turkey, and pork (16-18). EPA and DHA, however, are found in large quantities in fish and are typically more abundant in the cell membranes of humans consuming a 
"Mediterranean diet." Regardless of diet, however, the $\omega-6$ fatty acids compose the majority of fatty acids in human cell membranes.

Upon stimulation (such as insult or injury), AA, LA, EPA, and DHA are released from cell membranes via the activation of phospholipases, mainly cytosolic phospholipase $A_{2}\left(c P L A_{2}\right)$. Once released from the phospholipid bilayer, AA, LA, EPA, and DHA can be metabolized by three main enzymatic pathways: the cyclooxygenase pathway (COX-1 and COX-2), the lipoxygenase pathway (5-LOX, 12-LOX, and 15-LOX), and the cytochrome p450 pathway (CYP) (fig. 1.1).

\section{B. Cyclooxygenases (COX-1 and COX-2)}

The cyclooxygenase (COX) enzymes [also called prostaglandin endoperoxide $\mathrm{H}$ synthases (PGHS)] are responsible for the production of prostaglandins (PG) and thromboxanes $(T X)(19,20)$ (fig. 1.2). There are two isoforms of COX, COX-1 and COX-2 (PGHS-1 and PGHS-2, respectively) (20). These isoforms both catalyze the same two reactions: the cyclooxygenation (bisoxygenation) of $A A$ into prostaglandin $G_{2}\left(P_{G}\right)$ via addition of two molecules of $\mathrm{O}_{2}$ and the subsequent peroxidation of $\mathrm{PGG}_{2}$ to prostaglandin endoperoxide $\mathrm{H} 2$ $\left(\mathrm{PGH}_{2}\right)$ via reduction of two electrons (20). The cyclooxygenase reaction takes place in a hydrophobic channel in the enzyme's core while the peroxidase reaction takes place at a heme-containing active site at the protein's surface (20). 


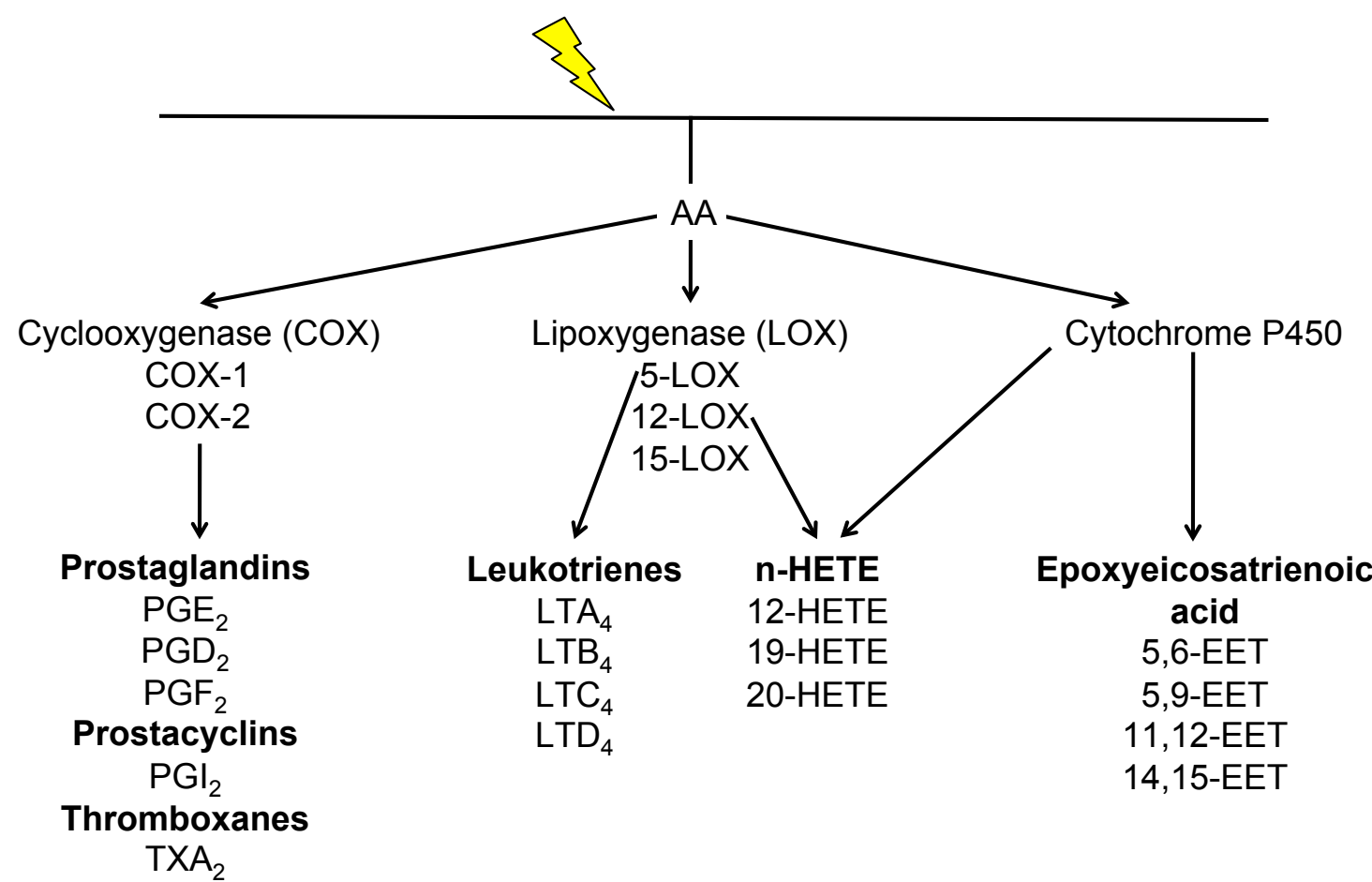

Fig. 1.1. The Major Metabolic Pathways of Eicosanoid Generation (21). Arachidonic acid (AA) (as well as LA, EPA, and DHA) can be metabolized by the cyclooxygenase (COX) pathways to generate prostaglandins (PG) and thromboxanes (TX), the lipoxygenase (LOX) pathways to generate leukotrienes (LT) and hydroxyeicosatetraenoic acids (HETE), or the cytochrome P450 (CYP) pathway to generate the epoxyeicosatrienoic acids (EET). (Adapted from Panigrahy, D. Cancer Metastasis Rev. 2010) (21). 


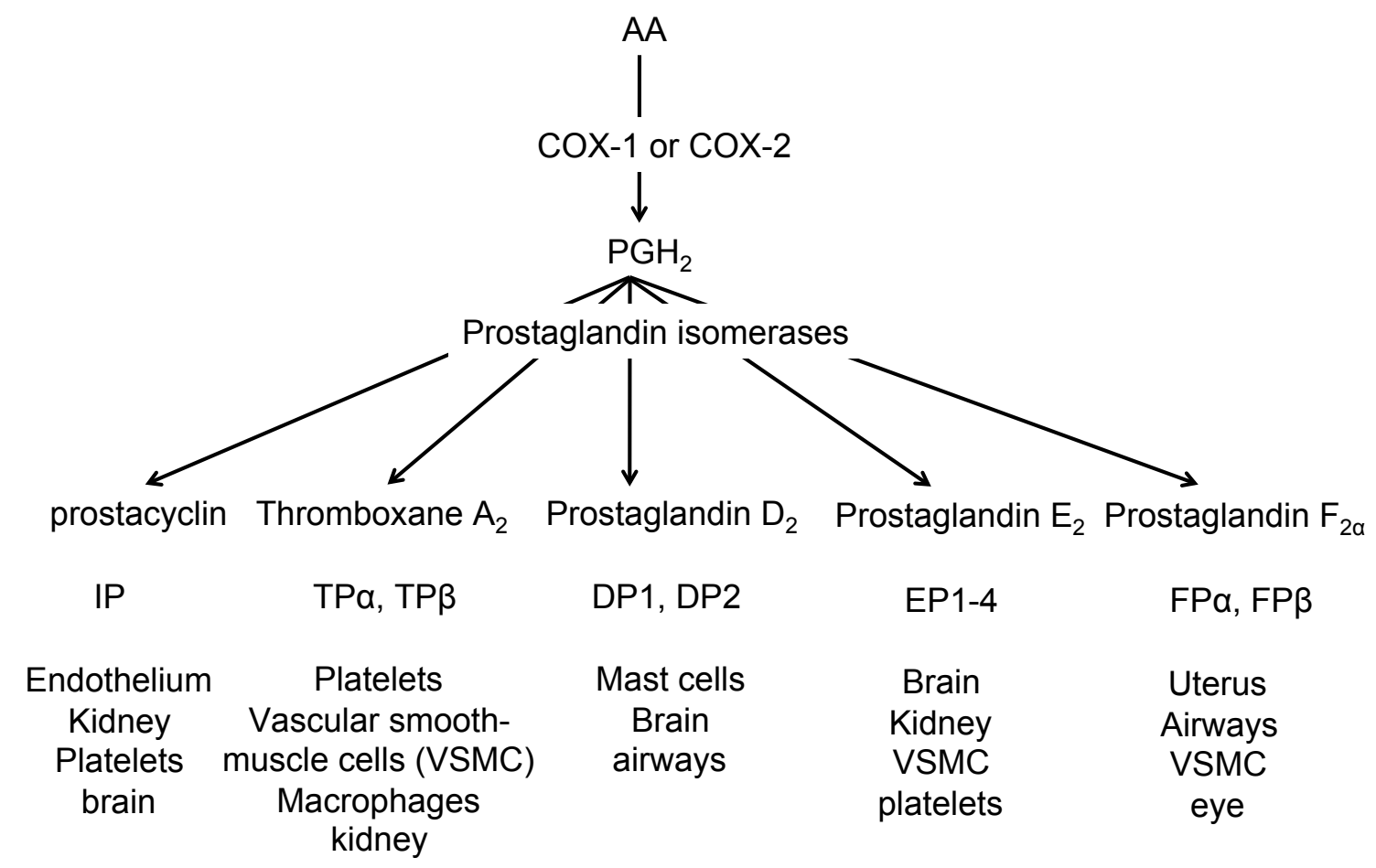

Fig. 1.2. Cyclooxygenase enzymes metabolize arachidonic acid into prostaglandins and thromboxanes (22). Cyclooxygenase coordinates the conversion of $A A$ into $P G G_{2}$ and $P G H_{2}$, which is further metabolized via specific synthases into prostaglandins and thromboxanes. The function of these molecules depends on the tissue localization and expression of cognate receptors. [Adapted from Fries, S. Hematology Am Soc Hematol Educ Program. 2005 (22)] 
Despite the fact that the function of COX-1 and COX-2 is enzymatically identical, the two enzymes are differentially regulated both spatially and temporally. While COX-1 localizes to the nuclear envelope, COX-2 is found in the smooth endoplasmic reticulum $(23,24)$. Furthermore, while the expression of COX-1 is constitutive in most tissues, COX-2 is typically stimulation-dependent (25-28). Thus, early prostaglandin synthesis is typically derived from COX-1 (29).

\section{Lipoxygenases (5-LOX and 12/15-LOX)}

The lipoxygenases are responsible for the production of leukotrienes (LT), hydroxyeicosatetraenoic acids (HETE), and for precursors of the lipoxins (LX), resolvins (Rv), and protectins (PD) (fig. 1.3). There are numerous forms of lipoxygenase, including 5-LOX, 12-LOX, and 15-LOX, the numerical value referring to the carbon atom of arachidonic acid which bears the hydroperoxy group upon oxygenation (30). In human cells, 12- and 15-LOX are separate enzymes; however, the homolog to these enzymes in murine cells is one enzyme, 12/15-LOX. 12- and 15-LOX are cytosolic $(31,32)$ while 5-LOX is found in both the nucleus and cytoplasm (33) and while 12/15-LOX is expressed in many immune and non-immune cells (34), 5-LOX expression is mostly confined to cells of myeloid lineage $(14,35)$. 


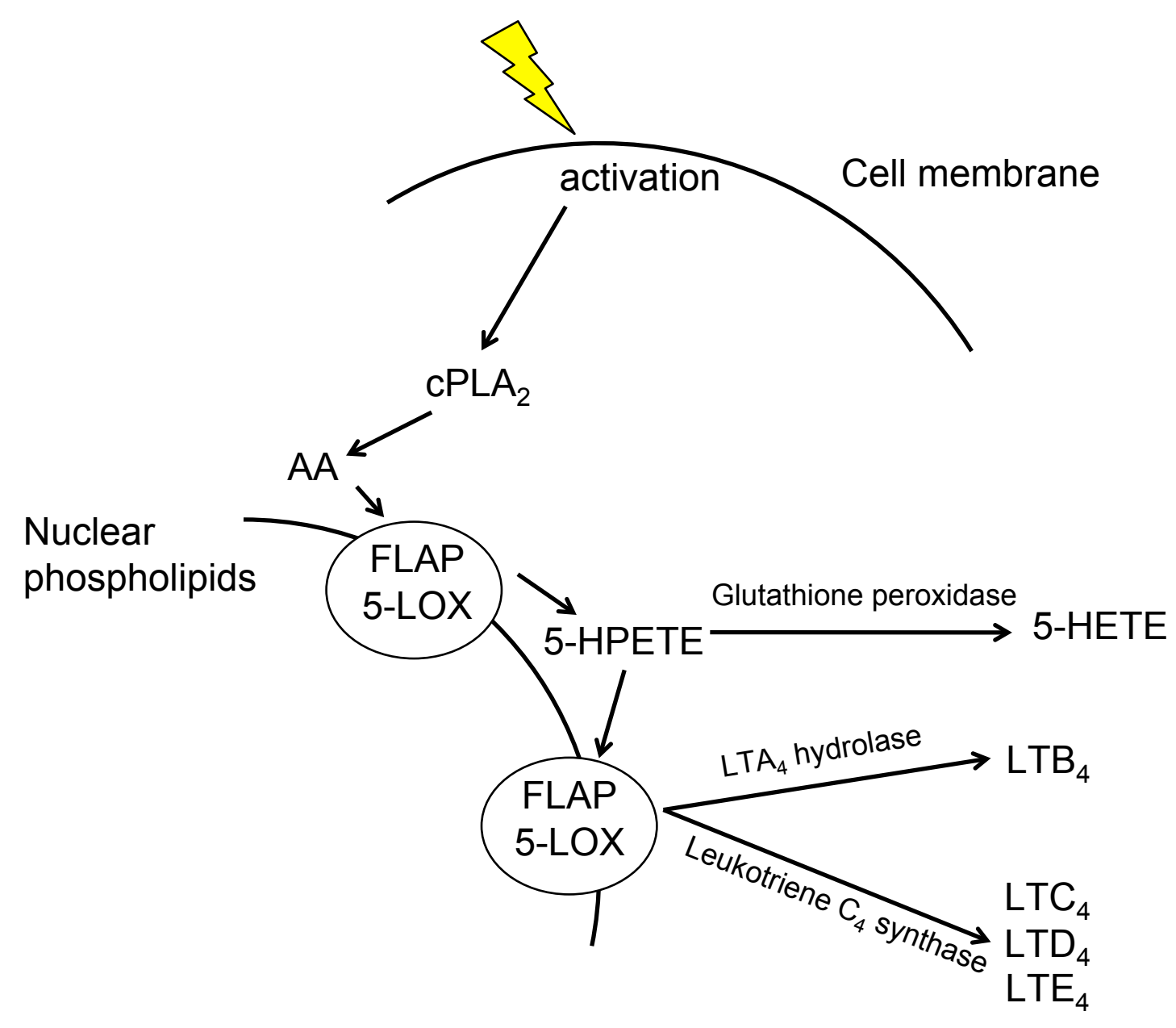

Fig. 1.3. Lipoxygenase enzymes metabolize AA into leukotrienes (36). 5lipoxygenase oxygenates AA into 5-HPETE, which is further converted into $\mathrm{LTA}_{4}$. $\mathrm{LTA}_{4}$ can be converted into a variety of LTs, including $\mathrm{LTB}_{4}, \mathrm{LTC}_{4}, \mathrm{LTD}_{4}$, and LTE$_{4 .}$ [Figure adapted from Steinhilber, D. Front Pharmacol. 2010. (36)]. 
In contrast to COX-2, 5-LOX activity does not require upregulation. Rather, 5-LOX activity requires the translocation of 5-LOX to the nuclear membrane and its association with five-lipoxygenase activating protein (FLAP). This process is stimulated by intracellular $\mathrm{Ca}^{2+}$ flux and activation of $\mathrm{CPLA}_{2}$ (33). Upon activation, 5-LOX oxygenates AA into 5-hydroperoxy-6,8,11,14eicosatetraenoic acid (5-HPETE) which is further converted into leukotriene $A_{4}$ $\left(\mathrm{LTA}_{4}\right)$ via the elimination of the hydroxyl moiety from the hydroperoxy group (37). LTA 4 can be further converted by $\mathrm{LTA}_{4}$ hydrolase $\left(\mathrm{LTA} \mathrm{A}_{4} \mathrm{H}\right)$ into leukotriene $\mathrm{B}_{4}\left(\mathrm{LTB}_{4}\right)$ (38-40). Alternatively, a glutathione transferase ( $\mathrm{LTC}_{4}$ synthase) can convert $\mathrm{LTA}_{4}$ into $\mathrm{LTC}_{4}(41)$. Further removal of a glutamic acid moiety via the action of a $\gamma$-glutamyl transpeptidase will yield $\mathrm{LTD}_{4}$ (42), which can be converted to $\mathrm{LTE}_{4}$ by the removal of glycine via a dipeptidase reaction (43). Collectively, $\mathrm{LTC}_{4}, \mathrm{LTD}_{4}$, and $\mathrm{LTE}_{4}$ are referred to as the cysteinyl leukotrienes (cysLT).

Many metabolites require the cooperation of multiple LOX enzymes for their production. The production of lipoxins (e.g.: LXA $)$, for example, requires both the 12/15-LOX and 5-LOX pathways and can occur via two mechanisms: 1) A 5-LOX-expressing myeloid cell can produce LTA $_{4}$ which is metabolized into $\mathrm{LXA}_{4}$ by a 12/15-LOX-expressing cell (e.g.: platelet) (44) or 2 ) a 12/15-LOX expressing cell can produce 15-HETE which is metabolized into $\mathrm{LXA}_{4}$ by 5 -LOX (45). These processes, wherein once cell produces a substrate that is metabolized by another cell, are examples of transcellular biosynthesis. 


\section{EICOSANOIDS IN INFLAMMATION}

Eicosanoids are known to play an important role in the regulation of homeostatic mechanisms in the gut (46), the kidney (47), the vasculature (48), and the cardiovascular and reproductive systems (49-51). Eicosanoids are also important mediators of both the innate and adaptive immune systems.

\section{A. Cyclooxygenase (COX) and inflammation}

Historically, the COX-1 isoform of COX has been associated with homeostatic regulation while the COX-2 isoform has been associated with inflammation. This is mostly due to the fact that COX-1 is constituitively expressed in most tissues $(25,28)$ while COX-2 expression, for the most part, is inducible (stimulation-dependent) $(26,27)$. Evidence that COX-2 is upregulated on immune cells during inflammation has further supported this view $(25,52,53)$. However, studies identifying COX-2 as important for the homeostatic regulation of the gut, brain, kidney, heart, and female reproductive system have somewhat challenged the categorization of COX-2 as "the inflammatory COX" $(28,54-56)$. Furthermore, data showing that COX-2-specific drugs (COXIBs) do not reduce the production of prostaglandins (PG) involved in inflammatory processes to the extent of dual COX-1/COX-2 inhibition [non-steroidal anti-inflammatory drugs (NSAIDs)] suggests that there exists at least a small role for COX-1 in the production of immune-modulating PGs (57-59).

The major PGs involved in inflammation are $\mathrm{PGD}_{2}$ and $\mathrm{PGE}_{2}$ and the net function of COX-2 expression is somewhat dependent on a balance of the 
production of these two metabolites. During an inflammatory response (e.g.: carrageenan-induced pleurisy), there are typically two peaks of COX-2 expression (fig. 1.4) (60). The first peak, which occurs $\sim 2$ hours postcarrageenan injection, is correlated with the onset of inflammation, the influx of polymorphonuclear leukocytes (PMNs) and $\mathrm{PGE}_{2}$ production. The second peak, which occurs between 24 and 48 hours, correlates with the resolution of inflammation, mononuclear cell influx, and $\mathrm{PGD}_{2}$ production (60). COX-2 expression during the second peak is also much higher. Thus, COX-2 functions as an immuno-regulatory enzyme in that it is involved in both the induction and resolution of inflammation.

$\mathrm{PGD}_{2}$ is typically classified as an anti-inflammatory eicosanoid, although it has also been implicated to be pro-inflammatory in models of asthma and hypersensitivity. It has been shown that $\mathrm{PGD}_{2}$ decreases dendritic cell (DC) migration to the draining lymph nodes $(\mathrm{dLN})$, which diminishes T-cell responses and promotes a worse outcome for viral infections (61). This has proved to be a hazardous side effect of naturally increased $\mathrm{PGD}_{2}$ production in the elderly, as it leads to decreased ability to combat infection. However, $\mathrm{PGD}_{2}$ also induces memory $\mathrm{CD}^{+}{ }^{+}$-lymphocyte trafficking during inflammation and exacerbates bronchial asthma by promoting vasodilation and increasing vascular permeability $(62,63)$.

Two major receptors for $\mathrm{PGD}_{2}$ exist: 1) chemoattractant receptor homologous molecules expressed on Th2 cells (CRTH2); and 2) D prostanoid 


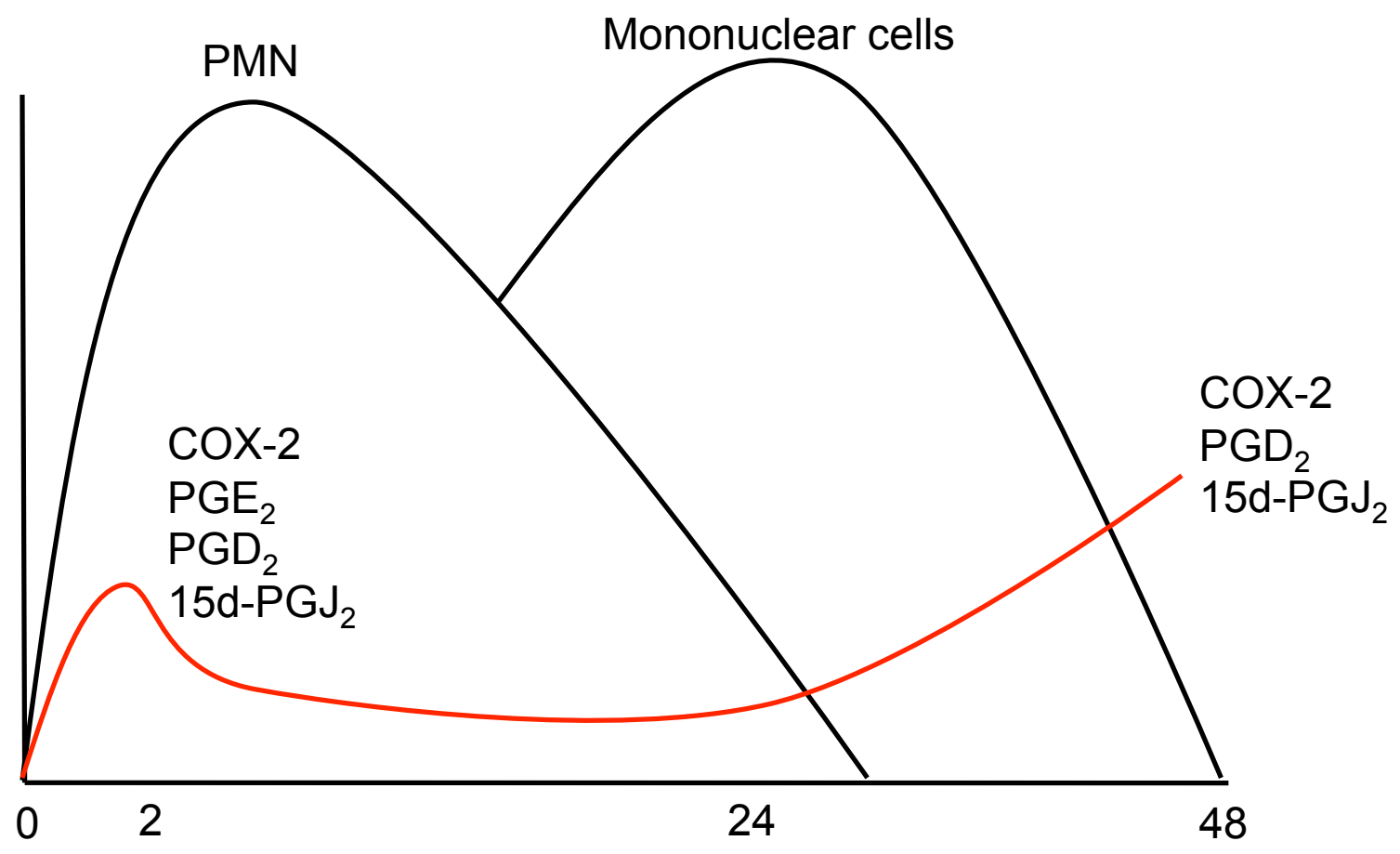

Days post-carrageenin injection

Fig. 1.4. COX-2 has a bimodal expression pattern during an inflammatory response (13). In a model of carrageenan-induced pleurisy, it was demonstrated that COX-2 expression occurs in two peaks. The first peak correlates with onset of inflammation, polymorphonuclear leukocyte infiltration, and $\mathrm{PGE}_{2}$ production, while the second peak correlates with the resolution of inflammation, mononuclear cell influx, and $\mathrm{PGD}_{2}$ production (60). [Adapted from Gilroy, D. \& Colville-Nash, P.R. J Mol Med. 2000 (13)]. 
receptor (DP). In a model of contact hypersensitivity (CHS) in mice, stimulation of the CRTH2 receptor promoted a hypersensitivity reaction while stimulation of the DP receptor had inhibitory effects on CHS (64). Thus, it appears that the contrasting pro- and anti-inflammatory functions of $\mathrm{PGD}_{2}$ may be receptormediated, making the function of $\mathrm{PGD}_{2}$ temporally- and tissue-regulated depending on which tissue expresses which receptor and when.

$15 d-P G J_{2}$ is the dehydration product of $P G D_{2}$ and is classified as an antiinflammatory mediator because it binds to the nuclear peroxisome proliferatoractivated receptor gamma (PPARY). This results in the inhibition of NF-KB activation and a subsequent decrease in the production of NF-KB-regulated proinflammatory metabolites (cytokines, chemokines, and eicosanoids) (65-68). The majority of studies examining $15 d-P G J_{2}$ as a ligand of PPARy, however, have been investigated in vitro. Thus, its role as a natural, endogenous ligand of PPARy and potent anti-inflammatory molecule in vivo remains inconclusive.

The function of $P G E_{2}$, like $P G D_{2}$, is context dependent and appears to be reliant on 1) the quantity of $\mathrm{PGE}_{2}$ produced (concentration) and 2) receptor expression (temporal- and tissue-dependent). Large concentrations (in the high nanomolar to micromolar range) of $\mathrm{PGE}_{2}$ are immunosuppressive. This has been demonstrated in cancer and tumor models, as tumor cells overexpress COX-2 and produce large quantities of $\mathrm{PGE}_{2}$ (69).

$\mathrm{PGE}_{2}$ exhibits a wide range of immunosuppressive effects, including the suppression of natural killer (NK) cell cytotoxicity (70); the induction of FOXP3 in T-cells and stimulation of IL-10 production by dendritic cells (DCs), which 
promotes T-regulatory (T-reg) cell development and expansion (71); the inhibition of granulocyte function (granule release and phagocytosis) (72); and the inhibition of phagocytosis and killing by alveolar macrophages (73). The ability of $\mathrm{PGE}_{2}$ to suppress NK cell, DC, granulocyte, and macrophage function as well as promote a T-regulatory phenotype, inhibit apoptosis (74), and promote tumor cell proliferation (69), make it a powerful player in the tumor microenvironment. COX-2 overexpression and $\mathrm{PGE}_{2}$ production are so commonly correlated with a poor prognosis in cancer diagnoses that many studies suggest that COX-2 should be a prognostic marker for cancers such as melanoma (75). On a more positive note, however, studies using NSAIDs and COXIBs are proving to be successful preventatives for various forms of cancer (76-81). One study reported that regular use of aspirin was able to decrease the long-term risk of several types of cancer as well as decrease the risk for metastasis (76).

Outside of the tumor microenvironment, however, $\mathrm{PGE}_{2}$ can also function as an immunosuppressor indirectly. For example, $\mathrm{PGE}_{2}$ is believed to mediate the "switch" from the production of 5-LOX to 15-LOX enzymes in human peripheral blood neutrophils (82). This switch is required for the subsequent production of the pro-resolution molecule, lipoxin $\mathrm{A}_{4}\left(\mathrm{LXA}_{4}\right)$. In a model of murine collagen-induced arthritis (CIA), COX-2 inhibition resulted in defective resolution of arthritis. Treatment with $\mathrm{PGE}_{2}$ restored resolution due to its stimulation of the production of $\mathrm{LXA}_{4}(83)$.

$\mathrm{PGE}_{2}$ is also important for the suppression of inflammation in response to infection of the gastro-intestinal $(\mathrm{Gl})$ tract (84). In the case of $\mathrm{Gl}$ infection, 
commensal bacteria stimulate the production of $\mathrm{PGE}_{2}$ by inflammatory macrophages, which inhibits neutrophil activation. In the absence of inflammatory macrophages or when $\mathrm{PGE}_{2}$ is inhibited, $\mathrm{GI}$ infection results in severe neutrophil-mediated pathology. Thus, commensal bacteria limit inflammation resulting from infection via stimulation of $\mathrm{PGE}_{2}$ production by inflammatory macrophages (84).

$\mathrm{PGE}_{2}$ is also renowned for the role it plays as an immuno-activator. It is able to promote the influx of neutrophils and macrophages via stimulation of the production of the chemokines CXCL1 and MCP-1, respectively $(85,86)$. It is also able to stimulate the influx and degranulation of mast cells (87-89). Furthermore, it promotes a skewing of CD4+ T-helper (Th) cell differentiation toward that of Th2 and Th17 responses. It does this by inhibiting the production of Th1promoting IFNy, but not Th2-promoting IL-4 or IL-5 $(89,90)$. It also inhibits the production of IL-12 by DCs while promoting the production of IL-23, leading to the production of IL-17-producing Th17 cells, which are instigators of arthritis, multiple sclerosis, Crohn's disease, and irritable bowel syndrome (91).

In terms of inflammatory diseases such as arthritis, $\mathrm{PGE}_{2}$ is believed to be pro-inflammatory. Deletion of microsomal PGE synthase (mPGES), which catalyzes the conversion of $\mathrm{PGH}_{2}$ to $\mathrm{PGE}_{2}$, results in attenuated arthritis in the CIA model (92). The contribution of $\mathrm{PGE}_{2}$ to arthritic inflammation in this model is due to defective development of a humoral immune response and skewing of the IL-23/IL-17 axis $(92,93)$. $\mathrm{PGE}_{2}$ also contributes to atherosclerotic inflammation via stimulation of arterial thrombosis (94) and experimental Crohn's 
disease via skewing of the T-helper cell compartment toward a Th17 phenotype (95).

The vast array of functions that $\mathrm{PGE}_{2}$ exhibits is due to the fact that it is able to signal through four separate receptors: E prostanoid receptor 1 (EP1), EP2, EP3, and EP4. Furthermore, EP3 has three isoforms (96). Regulation of these receptors is often tissue-, time-, and stimulation-dependent throughout the body, making the effects of $\mathrm{PGE}_{2}$ extremely circumstantial and highly regulated.

\section{B. Lipoxygenase (LOX) and inflammation}

The function of lipoxygenase products is much less ambiguous than the function of prostaglandins. For the most part, leukotrienes such as $\mathrm{LTB}_{4}$ and $\mathrm{LTC}_{4}$ are pro-inflammatory, while lipoxins, resolvins, and protectins are antiinflammatory.

$\mathrm{LTB}_{4}$ exerts many actions on innate immune cells. Perhaps most noteworthy is the requirement for $\mathrm{LTB}_{4}$ for phagocytosis and for the enhanced expression of the myeloid differentiation primary response 88 (MyD88) protein, both of which serve primary functions in the innate immune response.

Cyclic adenosine monophosphate (cAMP) is a second messenger with a wide range of functions, including the inhibition of phagocytosis. Although the exact mechanism by which cAMP regulates phagocytosis is unknown, it is suggested that it mediates F-actin polymerization (97-99). $\mathrm{LTB}_{4}$ signaling through its receptor BLT-1 leads to a decrease in CAMP and enhances phagocytosis of pathogenic and non-pathogenic particles (e.g.: Klebsiella 
pneumoniae, Streptococcus pneumoniae, immunoglobulin-coated sheep red blood cells) (100-102). $\mathrm{LTB}_{4}$ and $\mathrm{PGE}_{2}$ have contrasting roles in the regulation of phagocytosis. $\mathrm{PGE}_{2}$ signals through its $\mathrm{EP} 2$ receptor to stimulate an increase in cAMP, which inhibits phagocytosis $(73,103)$. $\mathrm{LTD}_{4}$ is also able to augment phagocytosis, however, it does this in a cAMP-independent fashion (104).

$\mathrm{LTB}_{4}$ also functions to enhance the expression of MyD88, an adapter molecule that is required for the signaling pathway downstream of all toll-like receptors (TLR) except TLR3 (105). LTB 4 signaling through BLT-1 inhibits suppressor of cytokine signaling 1 (SOCS1). SOCS1 suppresses MyD88 protein expression, thus inhibition of SOCS1 by $\mathrm{LTB}_{4}$ enhances MyD88 expression. This results in enhanced downstream signaling via NF-kB (105). $\mathrm{LTB}_{4}$ is also a potent neutrophil chemoattractant and mediates the migration of gamma delta Tcells in response to diverse stimuli $(106,107)$.

Due to its enhancement of immune cells functions, $\mathrm{LTB}_{4}$ is an important component of the innate immune response. Several studies have reported that in its absence [or in the absence of its cognate receptor(s)], immune function is reduced and the ability to clear infection is dampened. For example, 5-LOX knockout $\left(5-\mathrm{LOX}^{--}\right)$mice have increased mortality resulting from infection with Klebsiella pneumoniae due to reduced alveolar macrophage phagocytic and bactericidal abilities (108).

However, due to the potency of $\mathrm{LTB}_{4}$ as an immunoactivator, it also has a well-recognized role in pathogenic inflammation. It is responsible for the inflammation that develops in the $\mathrm{K} / \mathrm{BxN}$ serum transfer model of rheumatoid 
arthritis by mediating the recruitment of neutrophils, enhancing the invasive behavior of fibroblast-like synoviocytes (FLS), and promoting pannus tissue formation and joint erosion $(106,109,110)$. Furthermore, the cysteinyl leukotrienes are highly involved in the pathogenic mechanisms involved in asthma $(111,112)$.

Although $\mathrm{LTB}_{4}$ functions, for the most part, as an immunoactivator, it also plays a protective role in the gastric mucosa. $\mathrm{LTB}_{4}$ has two receptors; $B L T-1$, which mediates its effects on neutrophil recruitment and phagocytosis; and BLT2, about which less is known. The stimulation of $\mathrm{LTB}_{4}$ through BLT-2 on colon cryptic cells protects against dextran sodium sulfate (DSS)-induced colitis. It is believed that $\mathrm{LTB}_{4} / \mathrm{BLT}-2$ exerts this effect by enhancing colon epithelial cell barrier function (113).

5-LOX is also important for the production of the anti-inflammatory/proresolving metabolites lipoxins (LX), resolvins (Rv), and protectins (PD). Although the substrate for LX production is AA, the substrate for Rv and PD is EPA and/or DHA. Lipoxin $\mathrm{A}_{4}\left(\mathrm{LXA}_{4}\right)$ has a wide range of anti-inflammatory functions. It inhibits vascular endothelial growth factor (VEGF)-stimulation of VEGF receptor phosphorylation, which results in decreased phosphorylation of phospholipase Cy, extracellular signal regulated kinase (ERK) 1 and 2, and protein kinase B (Akt), which results in the inhibition of pro-inflammatory cytokines such as IL-6, TNFa, IFNy, IL-8, and ICAM and the enhancement of the anti-inflammatory cytokine IL-10 (114). Lipoxin $A_{4}$ and protectin D1 (PD1) are both independently able to dampen airway inflammation and hyper-responsiveness associated with 
asthma $(115,116)$ and EPA-derived resolvin E1 (RvE1) dampens $\mathrm{LTB}_{4} / \mathrm{BLT}-1$ signals on leukocytes and inhibits the recruitment of PMN (117).

\section{LYME ARTHRITIS AS A MODEL OF INFLAMMATION}

The Centers for Disease Control and Prevention (CDC) classifies arthritis as a class of diseases and conditions that affects the joints and connective tissue (rheumatic disease) with over 100 types, the most common of which is osteoarthritis (118). Arthritis is characterized by pain, aching, stiffness, and swelling of the joints and is the most common cause of disability in the United States. According to a 2013 CDC report, approximately 50 million (1 in 5) US adults have doctor-diagnosed arthritis and this number is expected to jump to 67 million by 2030 (118).

The most commonly used animal models of arthritis are chronic or autoimmune in nature, such as collagen-induced arthritis, collagen antibodyinduced arthritis, and $\mathrm{K} / \mathrm{BxN}$ serum transfer arthritis. These models are beneficial for the study of mechanisms involved in the development and peak of arthritis, but leave a very important gap in the knowledge about a typical arthritic response: how does arthritic inflammation resolve under normal circumstances? These models are dependent on the development of autoantibodies and autoimmunity. In experimental models, the resolution of these arthritides coincides with the degradation of these autoantibodies [e.g.: anti-glucose-6phosphate (GPI) autoantibodies transferred into recipient mice in the $\mathrm{K} / \mathrm{BxN}$ serum transfer model]. However, the relevance of an immune response to 
autoantibodies is not all encompassing for the study of rheumatic disease. For example, it is hypothesized that inflammation that develops during rheumatoid arthritis is instigated by autoantibodies to "rheumatoid factor (RF)." However, RF is only about $70 \%$ specific to rheumatoid arthritis and a number of other autoantibodies have failed to be diagnostic as well (119). Furthermore, it is difficult to translate results from animal models in which arthritis is instigated by a specific origin to human rheumatic disease in which the causes are often multifaceted and unidentified.

The fact that the cause is unknown in most cases of arthritis has made developing successful therapeutics and treatments quite difficult. With the discovery of novel anti-inflammatory/pro-resolving molecules ( $L X, R v$, and PD) has come an ideological shift in the study of arthritis. Whereas, in the past, research focused on identifying key pro-inflammatory molecules that aided in the development of arthritis (e.g.: TNFa, IL-6, $\mathrm{PGE}_{2}, \mathrm{LTB}_{4}$ ), it is now recognized that strategically targeting molecules that aid in promoting the resolution of arthritis could be beneficial. This ideological shift, however, has led to the necessity of models that allow the study of all phases of an inflammatory response: the development, the peak, and the resolution.

Lyme disease is the most common vector-borne disease in the United States and Europe $(120,121)$. Dr. Alan Steere and colleagues first described the disease in 1977 in an effort to identify the cause of an outbreak of juvenile rheumatic disease in Lyme, Connecticut (122). A spirochete isolated specifically from Ixodes ticks was identified as the causative agent by Dr. Willy Burgdorfer in 
1982 and was so-named Borrelia burgdorferi $(B b)$ (123). Human Lyme disease results from the bite of an infected Ixodes scapularis tick. Early manifestations of disease include mild flu-like symptoms and may include a diagnostic bulls-eye shaped rash. At this stage, disease is treatable with antibiotics such as Doxycycline. However, if allowed to progress, Lyme disease can manifest in a severe polyarthritis localizing to the large joints and severe carditis $(121,124)$.

After years of effort trying to develop a laboratory animal model for Lyme disease, a murine model was established by Dr. Steven Barthold and his colleagues at Yale University (125). The mouse model of Lyme arthritis mimics the human disease very well in that inoculation of mice with $B b$ results in joint and cardiac inflammation (126). Furthermore, human disease symptoms often appear cyclic in that they are remitting and recurring, a disease phenotype that is also present in the mouse model (127). In this mouse model, it is suggested that this phenomenon could either be due to neutralized $B b$ regaining pathogenicity or spirochetal antigen deposits persisting near cartilage $(128,129)$.

The exact mechanisms that mediate the development of Lyme arthritis and carditis remain elusive. However, it was identified early on that a genetic component was involved in the regulation of experimental Lyme disease development. In response to infection with $B b, \mathrm{C} 3 \mathrm{H} / \mathrm{He}$, SWR, and $\mathrm{CB} .17$ (SCID) mice develop severe arthritis while C57BL/6, BALB/c, and SJL mice appear resistant to disease $(130,131)$. Similarly, C3H/He, but not C57BL/6, mice are susceptible to carditis (126). Notably, $B b$ can be detected in the hearts 
and joints of all strains, indicating that the difference between the strains is in their genetic susceptibility to disease, not infection.

Production of the chemokine KC signaling through the CXCR2 receptor and subsequent recruitment of neutrophils is required for the development of Lyme arthritis (132-134) and neutrophils are the predominant infiltrating cell during the peak of arthritic disease (125) while macrophages predominate during Lyme carditis (135). Bb-specific antibodies are thought to mediate the clearance and eventual resolution of Lyme arthritis as SCID mice develop chronic inflammation and the passage of immune serum from infected mice results in the resolution of arthritic inflammation in recipient mice $(136,137)$. The development

of anti-Bb antibodies is T-cell-independent, as resolution is independent of MHC II, CD4+ T-cells, and CD40 ligand (138, 139).

Not only does murine Lyme arthritis offer an excellent model for a relevant human disease, but it also serves as a beneficial model of arthritis in which the induction, peak, and resolution of the inflammatory response are evident. The transient nature of this model and the fact that it does not rely on a specific autoimmune compartment make it an excellent tool for the study of arthritis.

\section{EICOSANOIDS IN LYME ARTHRITIS}

As eicosanoids comprise a major component of the innate immune response, it may be expected that they are heavily involved in the processes involved in Lyme arthritis, and this is indeed the case. COX-2 is upregulated in the joints of mice infected with $B b$ and treatment of mice with the COX-2 specific 
inhibitor 3-(3,4 difluorphenyl)-4-[4-(methylsulfonyl)phenyl]-2-(5H)-furanone (MF Tricyclic) results in lessened arthritis severity (140). However, while mice deficient in the enzyme COX-2 $\left(\mathrm{COX}-2^{-1-}\right)$ have slightly attenuated arthritis severity, this inhibition results in a chronic arthritis, suggesting that COX-2 metabolites play a role in the resolution of Lyme arthritis (141). $5-\mathrm{LOX}^{-/-}$mice exhibited a similar chronic phenotype (142), perhaps due to defective $\mathrm{LXA}_{4}$ production, which requires this pathway.

C57BL/6 mice deficient in COX-1 have no discernible defect in the development or resolution of arthritis, but are defective in their ability to form germinal centers and produce IL-6 and IL-17. These defects correlated with a defect in antibody class switching and reduced $B b$-specific immunoglobulin $G$ (IgG) production. Furthermore, sera from these mice had reduced bactericidal activity (143).

Lipidomic analysis of eicosanoids produced throughout the course of Lyme arthritis reveals that pro-inflammatory prostaglandins and leukotrienes are produced early during disease, while the peak of pro-resolving molecule (e.g.: $15 d-P G J_{2}$ and PD1) production correlates with the peak of inflammation, likely promoting the process of resolution (144). Lipidomic analysis of COX-2 ${ }^{-/-}$mice suggests that deletion of this enzyme also affects the lipoxygenase pathway, as COX $-2^{-/-}$mice have reduced production of numerous LOX metabolites (144). This may suggest that the chronic arthritis that arises in COX-2-- mice may be due to defective LOX signaling and thus defective production of the pro-resolving metabolites produced via this pathway. While this information confirms a role for 
eicosanoid enzymatic pathways in the regulation of Lyme arthritis, the elucidation of the individual eicosanoids important for these processes remains to be completed.

Inflammation is a vital process required for the protection of tissues from insult or injury. The resolution of inflammation, however, is an important component of the inflammatory response. Inflammatory responses that are defective in the process of resolution become pathogenic (chronic inflammatory disease). With a defined induction, peak, and resolution phase of inflammation, Lyme arthritis is an excellent tool for the study of inflammation. Eicosanoids are small, potent lipids that play a large role in the modulation of both the innate and adaptive immune compartments and are major regulators of inflammatory Lyme arthritis. Using murine Lyme arthritis as a model for the elucidation of mechanisms by which eicosanoids regulate inflammation and immunity will provide valuable insights into key pathways that may play a role in the development of chronic inflammation. A more thorough understanding of these pathways will aid in the identification of potential therapeutic targets for the treatment of chronic inflammatory diseases such as severe arthritis. 


\section{CHAPTER TWO}

\section{METHODS \& MATERIALS}

\section{ANIMALS}

\section{A. Control Mice}

$\mathrm{C} 3 \mathrm{H} / \mathrm{HeJ}$ mice were obtained from The Jackson Laboratories (Bar Harbor, $\mathrm{ME})$.

\section{B. $\mathrm{KC}^{-/-}$Mice}

$\mathrm{C} 3 \mathrm{H} \mathrm{KC}-1-$ mice were generated by Charles $\mathrm{R}$. Brown using speed congenics. B6/129 KC heterozygote males (a generous gift from Sergio Lira, Mount Sinai School of Medicine) were backcrossed onto the $\mathrm{C} 3 \mathrm{H}$ background for 5 generations. $\mathrm{C} 3 \mathrm{H} \mathrm{KC}$ heterozygote males and females were then crossed to create $\mathrm{C} 3 \mathrm{H} \mathrm{KC}{ }^{-/-}$mice.

\section{KRN, NOD, and K/BxN Mice}

KRN mice were a kind gift from Diane Mathis at Harvard Medical School. NOD mice were obtained from The Jackson Laboratories. KRN males were bred to NOD females in order to generate K/BxN pups. Pups were monitored for the development of arthritis. Retro-orbital eye bleeds were performed on pups that developed arthritis. Eye bleeds were performed once a week for five weeks on alternating eyes. Mice were sacrificed on the $5^{\text {th }}$ week via cardiac puncture. Serum was collected and stored at $-20^{\circ} \mathrm{C}$ for future use. 


\section{D. $\operatorname{coX}_{-2}^{-{ }^{--}}$Mice}

$\mathrm{COX}_{-2^{--}}$mice were purchased from The Jackson Laboratories (B6; 129S-

Ptgs $2^{\operatorname{tm} 1 \mathrm{Jed} / \mathrm{J}}$ ). Mice were backcrossed for ten generations onto the $\mathrm{C} 3 \mathrm{H} / \mathrm{HeJ}$ background.

\section{Housing and Experimental Conditions}

Mice were housed in a specific pathogen-free, environmentally stable (12 hour dark/light cycle, $23^{\circ} \mathrm{C}, 40-50 \%$ relative humidity) facility. Unless otherwise mentioned, mice were fed commercial rodent diet (Purina Mills) \& given water ad libitum. Mice were allowed 5 day acclimation periods prior to use in experiments. Experimental mice were age and sex matched if possible. All studies were conducted in accordance with the Animal Care and Use Committee of the University of Missouri.

\section{EXPERIMENTAL DIETS}

\section{A. Fish Oil Diets}

Fish oil and control diets were constructed by Kevin L. Fritsche (University of Missouri-Columbia) (145). Experimental diets were based on the AIN-93G rodent diet $(146,147)$ and were constructed so that the nutrient-to-energy ratio was not altered compared to normal rodent diet when modified to contain $18 \%$ fat. The "control" diet was supplemented with soy oil (SO) and the "experimental" diet was supplemented with a mixture of Menhaden fish oil (FO) and SO in a 4:1 ratio. SO was added to the FO diet in order to ensure that essential fatty acid 
requirements were met. Both diets were identical except major dietary fat sources and contained 375-g/kg corn starch, $230-\mathrm{g} / \mathrm{kg}$ casein, $110-\mathrm{g} / \mathrm{kg}$ sucrose, 57-g/kg a-cellulose, 40.2-g/kg AIN-93G mineral mix, 11.5-g/kg AIN-93G vitamin mix, $3.4-\mathrm{g} / \mathrm{kg}$ L-cysteine, $2.9-\mathrm{g} / \mathrm{kg}$ choline bitartrate, and $180-\mathrm{g} / \mathrm{kg}$ dietary fat sources. Dietary fats included in the chows were as follows (expressed as percent of total fatty acids in either the SO or FO diet, respectively): 0.2 or $4.5 \%$ myristic acid (14:0); 10.9 or $17.6 \%$ palmitic acid (16:0), 5.0 or $3.5 \%$ stearic acid (18:0); 23.8 or $16.8 \%$ oleic acid (18:1), 52.8 or $12.7 \%$ linoleic acid [18:2(n-6)]; and 6.4 or $1.6 \%$ a-linolenic acid [18:3(n-3)]. The FO diet also included $15.9 \%$ eicosapentaenoic acid (EPA) [20:5(n-3)]; 2.9\% docosapentaenoic acid [22:5(n3)]; $12.0 \%$ docosahexaenoic acid (DHA) [22:6(n-3)]; $7.1 \%$ palmitoleic acid [16:1(n-7)]; 1.6\% cis-vaccenic acid [18:19n-7)]; $2.7 \%$ stearidonic acid [18:4(n-3)]; and $1.0 \%$ eicosatetraenoic acid $[20: 4(n-3)]$. Fat sources included $0.02 \% \mathrm{wt} / \mathrm{wt}$ tertiary butylhydroquinone in order to prevent auto-oxidation. Mice were fed experimental diets for two weeks prior to infection with $B b$.

\section{B. Celecoxib Diets}

Celecoxib, a selective COX-2 inhibitor, was obtained from LKT Laboratories (St. Paul, MN) and incorporated into a normal rodent chow (Purina PicoLab 5053; Purina Mills, St. Louis, MO) at a dose of 1000 parts per million (ppm) by Research Diets (New Brunswick, NJ) $(141,148)$. Control animals were fed normal rodent chow. Mice were fed experimental diets for two weeks prior to infection with $B b$. 


\section{FATTY ACID ANALYSIS}

Serum and liver fatty acid extraction and analysis was performed by Dr. Kevin L. Fritsche $(145,149,150)$. Total cellular lipids were extracted from the serum and the liver (150) and resuspended in chloroform-methanol $(2: 1, v / v)$. The sample was applied to High Performance Thin-Layer Chromatography (HPTLC) Silica Gel 60 plates (Merck) and developed in hexane-diethyl etheracetic acid $(85: 15: 12, v / v / v)$ for 30 minutes. An air gun was used to remove solvent and then HPTLC plates were sprayed with 2',7'-dichlorfluorescein (2.5mm/L methanol). A UV lamp was used to visualize the phospholipid spot and scrape it into a $30-\mathrm{mL}$ screw-capped tube with a Teflon-lined cap. $20-\mu \mathrm{g}$ of 17:0 fatty acid methyl esters (FAME) were added as an internal standard. Transmethylation of fatty acids was achieved by the addition of $1.0-\mathrm{mL}$ of $0.5 \mathrm{~mol} / \mathrm{L}$ sodium hydroxide for ten minutes followed by $2-\mathrm{mL}$ of chloroform for 10 minutes. FAME were extracted by adding $0.75-\mathrm{mL} \mathrm{H}_{2} \mathrm{O}$. Samples were filtered through sodium sulfate and analyzed by a Hewlett-Packard Model 5890 Gas Chromatograph equipped with a $30-\mathrm{m} \times 0.25-\mathrm{mm}$ inner diameter-fused silica capillary column (Supelco) (149). Comparing the relative retention times to that of commercial standards enabled identification of extracted FAME. Data are expressed as moles per 100 moles of the total identified FAME (149).

\section{BACTERIA \& INFECTIONS}

A virulent, low-passage isolate of the N40 strain of $B b$ was used for in vivo infections and in vitro stimulations. Aliquots of $\mathrm{Bb} \mathrm{N} 40$ were stored at $-80^{\circ} \mathrm{C}$, 
thawed in a $37^{\circ} \mathrm{C}$ water bath via swirling, and cultured in $7.5-\mathrm{mL}$ BarbourStoenner-Kelly II (BSK) media (151) containing 6\% rabbit serum (Sigma-Aldrich) (cBSK). $B b$ were allowed to grow to log-phase for 5 days at $32^{\circ} \mathrm{C}$. The $B b$ culture was diluted 1:100 in BSK for enumeration using a Petroff-Hausser counting chamber and dark-field microscopy. For infection, $B b$ were diluted in BSK lacking rabbit serum to reach a concentration of $1 \times 10^{6} / \mathrm{mL}$. Both hind footpads of mice were inoculated with $50-\mu \mathrm{L}$ of the culture, resulting in a final infectious dose of $1 \times 10^{5} \mathrm{Bb} /$ mouse (or $5 \times 10^{4}$ per footpad). For stimulation of in vitro cultured cells, $B b$ centrifuged at $10,000 \times \mathrm{g}$ for 3 minutes (152) and resuspended in Dulbecco's Modified Eagle Medium (DMEM, Invitrogen).

\section{ASSESSMENT OF JOINT SWELLING}

Although some exceptions have been noted, ankle (tibiotarsal joint) swelling is typically a good indicator of the underlying immune response associated with Lyme arthritis (153). Metric calipers were used to measure swelling through the craniocaudal portion of the ankle joint. Ankle measurements were recorded on day 0 (prior to inoculation with $B b$ ) and once or twice weekly thereafter. Increases in ankle diameter were calculated by subtracting the day 0 measurement from subsequent measurements.

Mice transferred $\mathrm{K} / \mathrm{BxN}$ serum often developed joint swelling that was too severe to monitor via metric calipers. Therefore, clinical scores were also used in order to assess gross arthritis severity. Clinical scores were assigned as described by Monach, et al. (154) on a $0-3$ scale. A score of 0 indicated that 
the joint was non-arthritic. A score of 1 indicated either mild ankle/tendon sheath swelling or focal swelling of one or more digits. A score of 2 indicated medium swelling of the joint. This score indicated that foot swelling was leading to the shape of the foot being altered. Whereas in an un-swollen joint, the top of the foot (toes) is wider than the back of the foot (ankle), severe swelling would lead to the back of the foot being wider than the front of the foot. A score of 3 indicated maximum swelling, in which the entire foot was swollen.

\section{ASSESSMENT OF TISSUE PATHOLOGY \& IMMUNOHISTOCHEMISTRY}

Histological assessment of tissue inflammation was conducted by a boardcertified veterinary pathologist, Jennifer Hughes-Hanks. Hearts (sagittally bisected through both ventricles and atria) and joints were fixed in 10\% buffered zinc-formalin, mounted, and stained with hematoxylin and eosin (H\&E). Severity scores were on a 0 to 4 scale, with 0 representing no inflammation and 4 representing severe inflammation (involving $>50 \%$ of the sample). Inflammation type scores, representing the ratio of neutrophils to macrophages, were on a 0 to 4 scale and generated using the following criteria: $0=$ few neutrophils or macrophages; $1=<5 \%$ neutrophils, $>90 \%$ macrophages; $2=5-25 \%$ neutrophils, $>75 \%$ macrophages; $3=25-50 \%$ neutrophils, $50-75 \%$ macrophages; $4=>50 \%$ neutrophils, $<50 \%$ macrophages. Inflammation type scores and the criteria used to generate them are based on data suggesting which cells mediate the different phases of Lyme carditis and arthritis $(133,135)$ and are valuable for the 
histological assessment of the induction, peak, and resolution phases of an immune response.

\section{EXTRACTION OF DNA FROM TISSUES USING TRIZOL}

Tissues including heart, ear, ankle (tibiotarsal joint), and knee were excised from mice and snap-frozen in liquid nitrogen. In some instances, tissues were stored at $-80^{\circ} \mathrm{C}$ until DNA extraction. For DNA extraction, tissues were removed directly from liquid nitrogen into $2-\mathrm{mL}$ screw-top microcentrifuge tubes (Sarsedt) containing approximately $500-\mu \mathrm{L}$ Zirconia beads (Biospec Products) and 1-mL TRIzol (Invitrogen). Samples were minced using sterile scissors and then placed in a Mini Bead Beater (Biospec Products) and homogenized for 2 minutes, after which time they were allowed to cool to room temperature for approximately 5 minutes. $200-\mu \mathrm{L}$ Chloroform was added to the samples, the samples were vortexed for 10 seconds, and then allowed to incubate at room temperature for 10 minutes. Samples were then centrifuged at $12,000 \times \mathrm{g}$ for 30

minutes at $4^{\circ} \mathrm{C}$. Supernatant was removed and $500-\mu \mathrm{L}$ Back Extraction Buffer (4M guanidine thiocyanate, $50 \mathrm{mM}$ sodium citrate, q.s. to $100-\mathrm{mL}$ with $1 \mathrm{M}$ Tris $\mathrm{pH}$ 8.0) was added to the samples. The samples were then vortexed, allowed to incubate at room temperature for 15 minutes, and then centrifuged at $12,000 \times \mathrm{g}$ for 15 minutes at $4^{\circ} \mathrm{C}$. Samples were collected from centrifuge and kept on ice. Supernatant was removed to a $1.5 \mathrm{~mL}$ microcentrifuge tube and $400-\mu \mathrm{L}$ isopropanol was added. Samples were vortexed, incubated at room temperature for 5 minutes, and then centrifuged at $12,000 \times \mathrm{g}$ for 5 minutes at $4^{\circ} \mathrm{C}$. Supernatant was removed and the pellet was washed carefully with $500-\mu \mathrm{L} 75 \%$ 
ethanol. Ethanol was removed from the tube and the DNA pellet was dried overnight in a biosafety cabinet. DNA from the ears was dissolved in $100-\mu \mathrm{L}$ double distilled water $\left(\mathrm{ddH}_{2} \mathrm{O}\right)$, while DNA from all other tissues was dissolved in 200- $\mu \mathrm{L}$ double distilled water. DNA was quantified using a NanoSpec and diluted to a $50 \mathrm{ng} / \mathrm{mL}$ for use in PCR (132).

\section{QUANTIFICATION OF BORRELIA BURGDORFERI IN TISSUES}

Enumeration of $B b$ in tissues was achieved using $A B I$ Prism 7700 Sequence Detection System (Applied Biosystems) for quantitative multiplex realtime PCR. Murine DNA was detected using primers and a probe for the single copy mouse gene, nidogen (nido) [Forward: 5'-CCAGCC ACA GAA TAC CAT CC-3'; Reverse: 5'-GGA CAT ACT CTG CTG CCA TC-3' (155, 156); Internal oligonucleotide probe: 5'-VIC-AGC TCA GCC TGG CAG ACG CAA AGTTAMRA-3' (132)]. Bb DNA was detected using primers and a probe for bacterial flagellin (flag) [Forward: 5'-TCT TTT CTC TGG TGA GGG AGC T-3'; Reverse: 5'-TCC TTC CTG TTG AAC ACC CTC T-3'; Internal oligonucleotide probe: 5'FAM-AAA CTG C(TAMRA)TC AGG CTG CAC CGG TTC-3'] (157). PCR reactions consisted of $10 \mu \mathrm{L} \mathrm{ddH} 2 \mathrm{O}, 12.5-\mu \mathrm{L} 2 \mathrm{X}$ TaqMan Universal PCR MasterMix (Applied Biosystems), 0.25- $\mu \mathrm{L}$ forward primer (150 nM final concentration), $0.25-\mu \mathrm{L}$ reverse primer (150 nM final concentration), $0.25-\mu \mathrm{L}$ probe (100 nM final concentration), and 1.0- $\mu \mathrm{L}$ DNA [which is estimated to contain 1000 copies of nido (155)] for a final reaction volume of $25-\mu \mathrm{L}$, in accordance with the manufacturer's protocol. The reaction proceeded at $50^{\circ} \mathrm{C}$ 
for 2 minutes, $95^{\circ} \mathrm{C}$ for 10 minutes, and with 45 cycles of $95^{\circ} \mathrm{C}$ for 15 seconds and $60^{\circ} \mathrm{C}$ for 1 minute. DNA isolated from a pure culture of $B b$ was used as a positive flag control, DNA isolated from uninfected murine tissue was used as a positive nido control, and a reaction containing no DNA was used as a negative control. $B b$ DNA in each sample was quantified by comparing the $\Delta \Delta C_{T}$ values obtained to a standard curve consisting of known numbers of $B b$. Normalization of mouse DNA in each sample was conducted similarly by comparing the $\Delta \Delta \mathrm{C}_{\mathrm{T}}$ values obtained to a standard curve consisting of dilutions of murine DNA from the tissue in question (ear, ankle, knee, etc.) (132). Each sample was run in duplicate. Copies of flag were normalized to copies of nido from in the same tube and results are expressed as copies of flag per 1000 copies of mouse nido.

\section{QUANTIFICATION OF TISSUE CYTOKINE \& CHEMOKINE PRODUCTION}

Tissues including heart, ankle (tibiotarsal), and knee were excised from mice and snap-frozen in liquid nitrogen as described (132). In some instances, tissues were stored at $-80^{\circ} \mathrm{C}$ until protein isolation occurred. Tissues were removed from liquid nitrogen and wrapped in foil. Foil-wrapped samples were then dipped in liquid nitrogen and smashed with a hammer. Pulverized tissue was then transferred into 2-mL screw-top microcentrifuge tubes (Sarstedt) containing 1-mL Hank's Buffered Saline Solution (HBSS) and Protease Inhibitor Cocktail (1:1000; company) (HBSS + PIC). Samples were kept on ice at all

times. Samples were sonicated at $60 \%$ for 20 seconds and then centrifuged at $8000 \mathrm{rpm}$ for 10 minutes at $4^{\circ} \mathrm{C}$. Supernatant was removed from samples and 
filtered using a 3-mL syringe attached to a $45-\mu \mathrm{m}$ filter and collected into a fresh 2-mL screw-top microcentrifuge tube. Samples were q.s. to 1.5-mL with HBSS + PIC. Tissue protein was quantified using Pierce bicinchoninic acid (BCA) Protein Assay Reagent (Thermo Scientific). Tissue cytokines and chemokines were quantified via Enyzme Linked Immunosorbent Assay (ELISA). KC, LIX, MIP-2, and TNF- $\alpha$ were detected using R \& D DuoSet kits (R \& D Systems) and IL-6 and IL-1 $\beta$ were detected using BD OptEIA kits (BD Biosciences). Cytokine and chemokine quantities were normalized to total tissue protein and results are expressed as picograms cytokine or chemokine per milligram tissue protein.

\section{EXTRACTION OF TISSUE EICOSANOIDS (144)}

Ankle (tibiotarsal joint) tissue was excised from mice and snap-frozen in liquid nitrogen as described (144). In some instances, tissues were stored at $80^{\circ} \mathrm{C}$ until eicosanoid extraction occurred. Tissue was removed from liquid nitrogen and wrapped in foil. Foil-wrapped samples were dipped in liquid nitrogen and smashed with a hammer. Smashed samples were again dipped in liquid nitrogen, and frozen tissue was transferred to a $16 \times 100 \mathrm{~mm}$ glass test tube filled with 3-mL ice-cold $50 \%$ ethanol, $50-\mu \mathrm{L}$ eicosanoid internal standard (supplied by the lab of Dr. Edward A. Dennis, University of California-San Diego), and 10- $\mu \mathrm{L}$ antioxidant cocktail $(0.2 \mathrm{mg} / \mathrm{mL}$ butylated hydroxytoluene, 0.2 $\mathrm{mg} / \mathrm{mL}$ EDTA, $2 \mathrm{mg} / \mathrm{mL}$ triphenylphosphine, $2 \mathrm{mg} / \mathrm{mL}$ indomethacin in a solution of 2:1:1 methanol:ethanol:water) located on a tared scale in order to obtain tissue weight. Test tube was capped and samples were kept on ice at all times. 
Samples were vortexed, incubated at $-20^{\circ} \mathrm{C}$ for 72 hours, and centrifuged at 3500 rpm for 30 minutes. Clear, ethanolic supernatant was removed to a $12 \times 75 \mathrm{~mm}$ glass test tube and dried under nitrogen gas. Dried samples were stored at $20^{\circ} \mathrm{C}$ and shipped on dry ice to Darren S. Dumlao in Dr. Edward A Dennis' lab at the University of California-San Diego for eicosanoid quantification.

\section{LC, MS, \& EICOSANOID QUANTIFICATION}

Lipidomic analyses were performed by Dr. Darren S. Dumlao in the lab of Dr. Edward A. Dennis at the University of California-San Diego (145) using a mass spectrometry (MS)-based approach (158). Liquid chromatography (LC)grade solvents were from EMD Biosciences. Strata-X Solid-phase Extraction Columns (Phemononex) were used to extract eicosanoids from dried samples into $1.0-\mathrm{mL}$ methanol. $40-\mu \mathrm{L}$ of the sample was separated by LC using a Synergy C18 Reverse-phase HPLC Column (Phemononex). Eicosanoids were detected using a tandem quadrupole MS (ABI 4000 Q-TrapR, Analyst 1.5 software, Applied Biosystems) via scheduled multiple reaction monitoring (MRM) in negative ion-mode (145). Comparing the MRM signals and LC retention times to those of purified standards (Cayman Chemicals and Biomol) enabled identification of extracted eicosanoids. Quantity of eicosanoid was standardized to total tissue weight and results are expressed as picograms eicosanoid per milligram tissue. 


\section{TREATMENTS}

$15 d-P G J_{2}, P G_{2}$, and Butaprost were all purchased from Cayman Chemical (Ann Arbor, Ml). All treatments were made fresh prior to administration. $60-n g$ of $16 \mathrm{~d}-\mathrm{PGJ}_{2}$ in $100-\mu \mathrm{L}$ PBS was delivered subcutaneously to mice on days $3-14$ post-infection. $1.0-\mu \mathrm{g}$ of $\mathrm{PGE}_{2}$ in $50-\mu \mathrm{L}$ of PBS was delivered subcutaneously to mice daily throughout the course of a 35-day infection. 5-uM Butaprost in 100- $\mu$ L PBS was delivered intraperitoneally on days $5-7$ and $12-15$ post-infection. PBS served as a vehicle control in all studies.

\section{STATISTICAL ANALYSES}

Bacterial loads are expressed as individual data points with bars indicating median values. Cytokine and chemokine data are expressed as means \pm standard deviation. Statistical significance of group means was analyzed using the Student's $t$ test (ELISA \& EIA results) or the Mann-Whitney rank sum test (severity scores) with Prism 6 (GraphPad Software) $(134,145)$. Significance levels were set at an $\alpha$ of 0.05 . Eicosanoid values in the lipidomic studies done in collaboration with D. Dumlao and E. Dennis were filtered using a Grubb's test to remove outliers. Values in these studies represent means \pm standard error of the mean and significance was determined by 2-way ANOVA $(P<0.05)$. Significance for specific comparisons made in the collaborative studies was determined by ANOVA and the Dunnett's post hoc test (metabolite changes over time on specific diet) or by Bonferroni's post hoc multiple comparison test (metabolite changes between diets) (145). 


\section{CHAPTER THREE}

\section{KC (CXCL1) MEDIATES THE DEVELOPMENT OF EXPERIMENTAL LYME \\ DISEASE}

\section{INTRODUCTION}

The development of experimental Lyme disease is mediated by innate immunity $(159,160)$ suggesting that genetic factors determining susceptibility or resistance regulate this compartment. In 2003, Brown, et al. surveyed the production of a panel of chemokines and cytokines in genetically susceptible $(\mathrm{C} 3 \mathrm{H} / \mathrm{HeJ})$ and resistant $(\mathrm{C} 57 \mathrm{BL} / 6)$ mice and found that the chemokines KC (CXCL1) and monocyte chemoattractant protein-1 (MCP-1; CCL2) were upregulated in $\mathrm{C} 3 \mathrm{H} / \mathrm{HeJ}$ compared to C57BL/6 mice (132). Infection of mice deficient in CCR2 (the receptor for CCL2) had no affect on disease severity in $\mathrm{C} 3 \mathrm{H} / \mathrm{HeJ}$ or C57BL/6 mice (132). However, infection of $\mathrm{C} 3 \mathrm{H} / \mathrm{HeJ}$ mice deficient in CXCR2 (the receptor for KC) resulted in attenuated arthritis severity, while deficiency of CXCR2 on the C57BL/6 background had no effect on disease (132). These data suggested that the ability of mice to develop disease in response to $B b$ infection was mediated by genetic factors regulating the production of CXCR2 ligands. Signaling through CXCR2 mediates the recruitment of neutrophils and in its absence neutrophil extravasation into joint tissue was drastically reduced (132). This suggests that in susceptible mouse strains, the recruitment of neutrophils is required for disease. 
The results from Brown, et al. 2003 identified a role for CXCR2 in the recruitment of neutrophils and subsequent development of Lyme arthritis in response to infection with $B b$. Three ligands mediate their effects through CXCR2: KC (CXCL1); macrophage inflammatory protein 2 (MIP-2); and LIX (CXCL5). Although KC was expressed in higher quantities in susceptible mouse strains than resistant mouse strains, it was unclear whether this chemokine alone, signaling through CXCR2, mediated the development of arthritis. Furthermore, while the inhibition of neutrophil recruitment via deletion of CXCR2 defined a role for this process in Lyme arthritis development, the effect of inhibiting neutrophil recruitment on Lyme carditis had not been determined.

The study described herein identified a critical role for the chemokine KC in the recruitment of neutrophils to sites of infection and subsequent development of Lyme arthritis and carditis.

\section{RESULTS}

\section{A. KC mediates edema resulting from infection with Borrelia burgdorferi.}

Neutrophil recruitment is known to be required for a variety of arthritis models, including CIA (161), CAIA (162), $\operatorname{K/BxN}(109,163,164)$, adjuvantinduced arthritis (165), and streptococcal cell wall-induced arthritis (166). Neutrophils are also known to play a critical role in the development of human RA $(167,168)$. Brown, et al. reported that absence of the potent chemokine receptor CXCR2 resulted in reduced neutrophil recruitment to the joint and attenuated Lyme arthritis (132). As stated above, CXCR2 has three known 
ligands: KC; MIP-2; and LIX. KC was expressed in greater amounts in the joints of Lyme arthritis susceptible mice $(\mathrm{C} 3 \mathrm{H} / \mathrm{HeJ})$ compared to Lyme arthritis resistant mice (C57BL/6) (132), hinting that the KC/CXCR2 axis mediates the development of Lyme arthritis.

To investigate the role of KC in Lyme arthritis, we infected wild type $\mathrm{C} 3 \mathrm{H} / \mathrm{HeJ}(\mathrm{WT})$ and $\mathrm{C} 3 \mathrm{H} \mathrm{KC}^{-/-}$mice with $5 \times 10^{4}$ of a virulent, low-passage isolate of $B b \mathrm{~N} 40$. We used metric calipers to monitor ankle swelling, a typical symptom arising from infection with $B b$, for 21 days. WT mice exhibited a typical course of disease, with progressive joint swelling following infection leading to a peak at around 17 days post-infection (fig. 3.1). $\mathrm{KC}^{-/-}$mice, however, exhibited attenuated ankle swelling. Ankle edema was significantly reduced at days 14 and 17 post-infection compared to their WT counterparts. This indicated that KC (most likely signaling through CXCR2) mediates ankle swelling associated with Lyme arthritis.

\section{B. KC mediates Lyme arthritis severity.}

Although joint edema is typically indicative of the underlying inflammatory response associated with Lyme arthritis, some exceptions have been noted (153). Therefore, to more thoroughly evaluate joint inflammation, we used H \& E to stain joint sections taken on days 11 and 21 post-infection. We assessed arthritis severity on a $0-4$ scale, where 0 represented no inflammation and 4 represented severe inflammation. $\mathrm{KC}^{-/-}$mice exhibited significantly less arthritis severity on days 11 and 21 post-infection (fig. $3.2 \mathrm{~A}$ ), indicating that KC mediates the severity of inflammation resulting from infection with $B b$. 


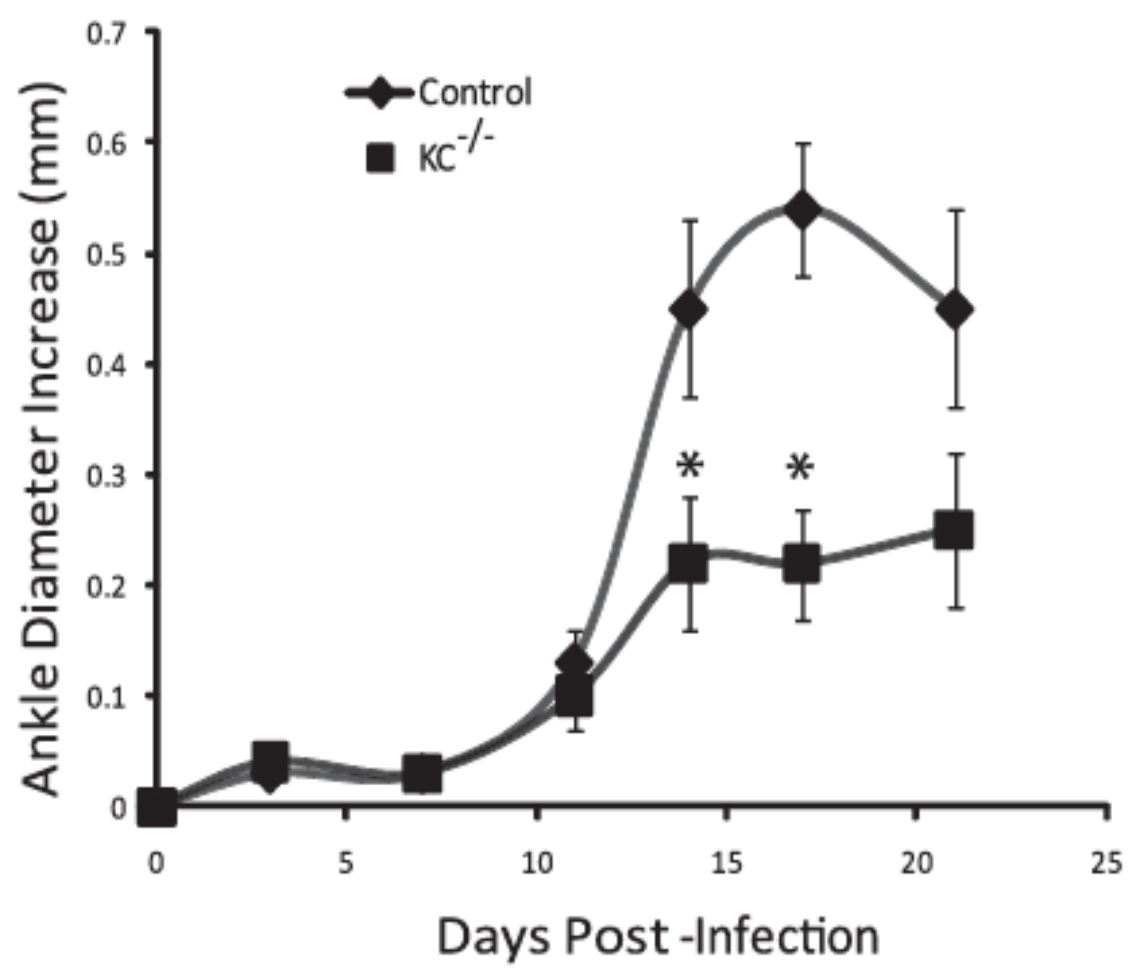

Fig. 3.1. $\mathrm{KC}^{-/-}$mice have reduced joint edema in response to infection with Borrelia burgdorferi (134). WT and $\mathrm{KC}^{--}$mice were infected with $5 \times 10^{4} \mathrm{Bb}$ via footpad inoculation. Disease was allowed to progress for 21 days. Throughout the course of infection, ankle swelling was monitored using metric calipers. Changes in ankle diameters were calculated by subtracting day 0 measurements from subsequent measurements. Data are representative of four separate experiments, 8 mice per group. Results are expressed as means \pm SEM and * indicates a significant difference from control at the same time point $(p<0.04)$. 
A

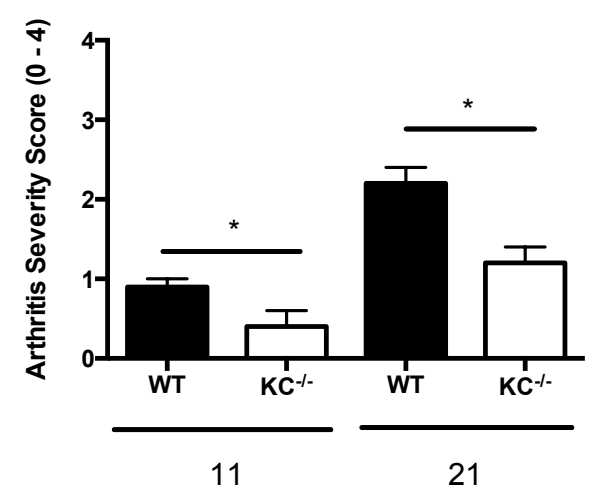

B

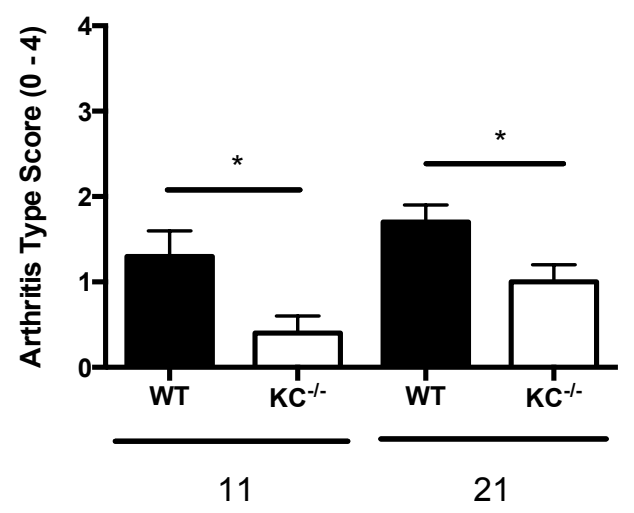

Fig. 3.2. $\mathrm{KC}^{-/-}$mice exhibit attenuated arthritis severity and altered joint cell infiltrate (134). Joint sections from mice at days 11 and 21 post-infection were stained with H\&E and arthritis severity (A) and type scores (B) were generated on a scale of $0-4$. Eight mice were in each group, data shown are means \pm SEM, and * indicates a significant difference from WT at the same time point ( $p$ $<0.05$ ). Assessment of histological sections and subsequent determination of arthritis severity scores and arthritis type scores was conducted by Jennifer Hughes-Hanks. 
Based upon the type of cells that accumulated during joint inflammation, we were able to generate arthritis type scores. These scores were based on the ratio of neutrophils to macrophages, as these are the two innate immune cells that predominate during Lyme arthritis (130). Arthritis type scores are on a scale of $0-4$, where 0 indicates few neutrophils or macrophages, 1 indicates a predominance of macrophages, and 4 indicates a predominance of neutrophils. It is believed that neutrophils mediate the early stages and development of arthritis and that macrophages predominate during the late stages and resolution of arthritis (130). Therefore, a higher type score (more neutrophils) suggests acute inflammation, while a lower type score (more macrophages or fewer immune cells in general) suggests low levels of inflammation and resolution of disease. $\mathrm{KC}^{-/-}$mice had significantly lower arthritis type scores, indicating that neutrophil infiltration was reduced in the joints of these mice (fig. 3.2 B). This data suggested that KC mediated neutrophil infiltration into the joints during Lyme arthritis.

\section{Absence of KC leads to reduced neutrophil and macrophage joint infiltration.}

To better assess the cell types present in the joints of WT and $\mathrm{KC}^{-/-}$during Lyme arthritis, we used immunohistochemical staining of joint sections to identify neutrophils and macrophages (RB6-8C5 and F4/80, respectively). Significantly fewer RB6-8C5-labelled neutrophils were present in the joints of $\mathrm{KC}^{-/-}$mice compared to WT counterparts at day 21 post-infection (fig. 3.3) confirming the 


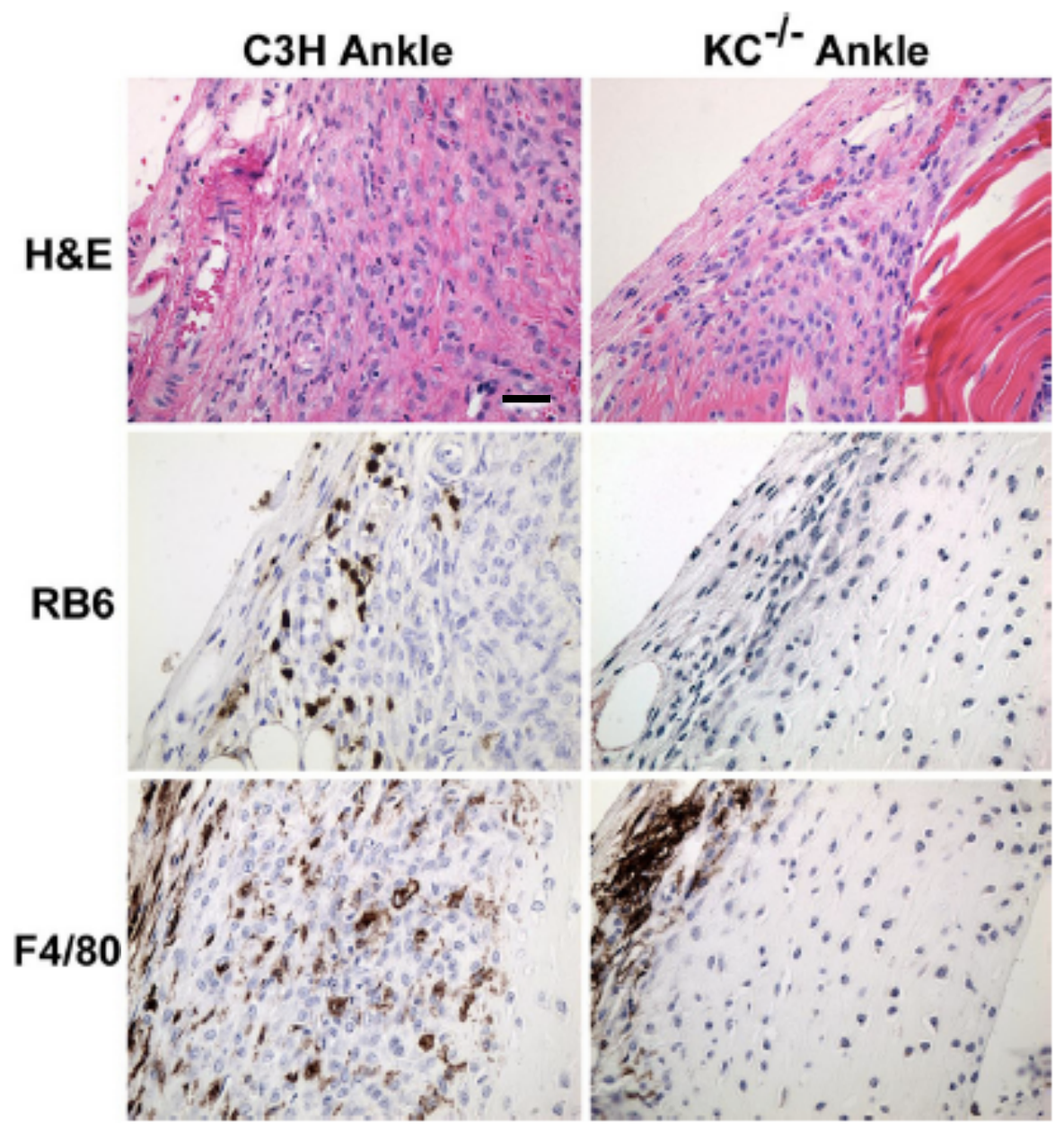

Fig. 3.3. $\mathrm{KC}^{-/-}$mice have significantly reduced neutrophil and macrophage infiltration into infected joint tissue (134). Joint sections of WT and $\mathrm{KC}^{-/-}$mice were stained with the neutrophil marker RB6-8C5 (RB6) or the macrophage marker F4/80. Representative sections from day 21 post-infection are displayed above. Magnification X100. Bar $=50 \mu \mathrm{m}$. Assessment of tissue pathology and joint cell infiltrate was conducted by Jennifer Hughes-Hanks. 
requirement for $\mathrm{KC}$ for the recruitment of neutrophils to $B b$-infected joints. Somewhat surprising was the finding that F4/80-labelled cells (presumably macrophages) were also greatly reduced in number in the joints of $\mathrm{KC}^{-/-}$mice (fig. 3.3). This data suggests that $\mathrm{KC}$ is important for the recruitment of macrophages to the joint; however, whether this is a direct effect of $\mathrm{KC}$ or whether the defect in neutrophil recruitment in the absence of KC leads to a defect in subsequent production of macrophage chemotactic factors and thus macrophage recruitment is unknown.

\section{Absence of KC does not affect the ability of spirochetes to be cleared from joints of $B b$-infected mice.}

Neutrophils are known to play a large role in the clearance of $B b$ from tissues (169-171). As neutrophils were drastically reduced in the joints of $\mathrm{KC}^{-/-}$ mice, we hypothesized that the quantity of $B b$ in joint tissue would be increased. We used quantitative Real-Time PCR to detect copies of the Bb flagellin B (flab) gene in DNA from joint tissue at days 10, 21, and 35 post-infection and normalized them to 1,000 copies of the murine gene Nidogen (nido). Although at days 10 and 35 post-infection the copies of $B b$ DNA in joints from $\mathrm{KC}^{-/-}$mice appeared to trend higher compared to Bb DNA in WT joints, these differences did not reach statistical significance (fig. 3.4 A). As $B b$ tends to migrate to the ears of infected mice (125), we also assessed $B b$ numbers in ear tissue and found no difference between the numbers of $B b$ DNA in $\mathrm{KC}^{-/-}$tissue when compared to WT tissue (fig. 3.4 B). This data implies that although $\mathrm{KC}^{-/-}$mice exhibited a defect in 
A

Ankles

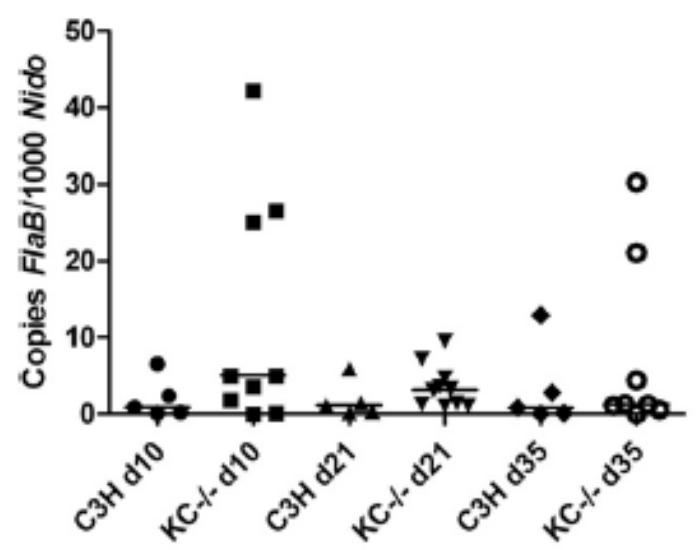

Ears

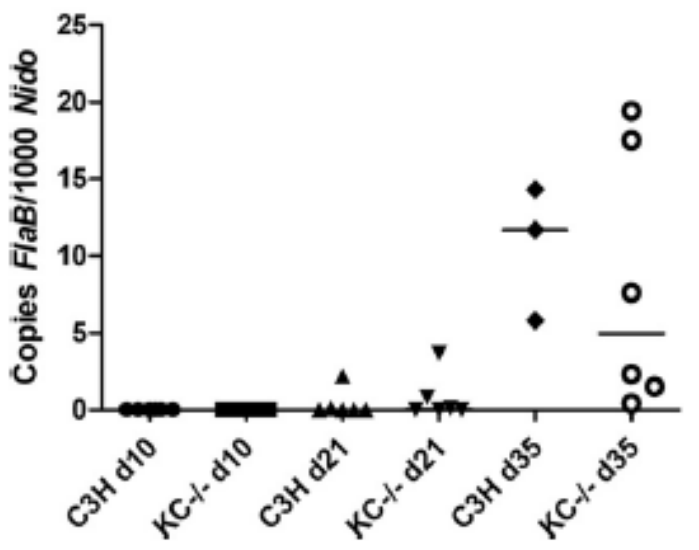

Fig. 3.4. $\mathrm{KC}^{-/-}$mice are able to clear $B b$ infection as well as WT mice (134). Joints and ears were removed from mice at days 10,21 , and 35 post-infection. Spirochete DNA was detected using quantitative Real Time PCR. Copies of the $B b$ flagellin gene $(F l a B)$ were normalized to 1000 copies of the mouse gene nidogen (Nido). Data are representative of two separate experiments and bars indicate median values. 
the recruitment of both neutrophils and macrophages to infected joints, they were not impaired in their ability to clear infection.

\section{E. Joint cytokine and chemokine expression is unaffected by the absence of KC.}

Besides contributing to the clearance of pathogens by phagocytosis, neutrophils also produce cytokines $(109,172-175)$, lipids $(109,176)$, and other immune factors that aid in the development of an immune response to infection $(173,177)$. In an effort to assess whether the drastic reduction in neutrophils and macrophages in the joints of $\mathrm{KC}^{--}$mice had an effect on the cytokine milieu, we extracted protein from the joints of infected mice at days 11 and 21 post-infection and measured the expression of three classical cytokines: IL-6; TNF- $\alpha$; and IL1ß. Despite the fact that $\mathrm{KC}^{-/-}$mice had far fewer neutrophils and macrophages in the joints at these time points, the expression of these cytokines was no different than in WT mice (fig. 3.5 A). These results indicated that although KC deficiency affected the joint immune cell milieu, it did not affect the ability of these

mice to produce relevant cytokines similar to WT levels. These results also suggest that either other cells present in the joints during infection (fibroblasts, Bcells, monocytes, non-F4/80-expressing macrophages, non-Gr-1-expressing polymorphonuclear cells (132), etc.) were producing the majority of these cytokines or that the limited number of neutrophils and macrophages present were able to produce quantities of these cytokines comparable to those 
A
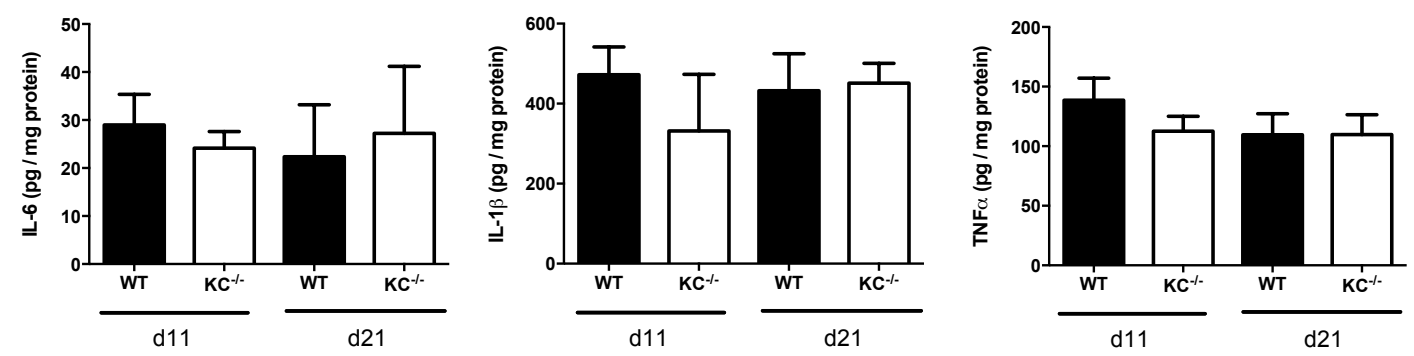

B
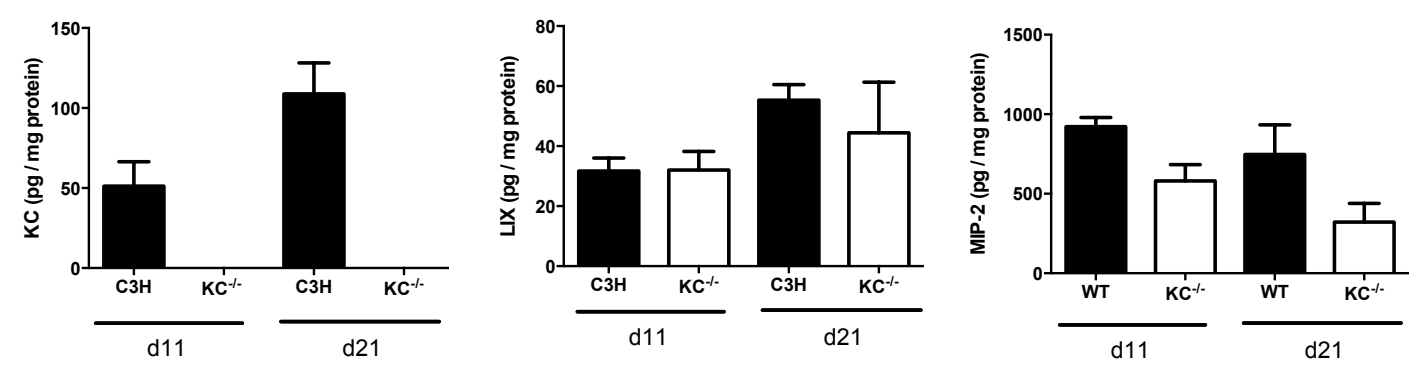

Fig. 3.5. $\mathrm{KC}^{-1-}$ mice are able to effectively produce cytokines and chemokines to WT levels (134). On days 11 and 21 post-infection, joint tissue was removed and protein was extracted. Levels of the cytokines IL-6, IL-1 $\beta$, and TNF- $\alpha(\mathbf{A})$ and the chemokines KC, LIX, and MIP-2 (B) were quantified using ELISA. No significant differences were noted between $\mathrm{KC}^{-1-}$ mice and WT mice at any time point. 
expressed in WT joints. LIX and MIP-2 are two other chemokines that bind CXCR2 and aid in the recruitment of neutrophils and macrophages to sites of infection. We were curious as to whether the expression of these two chemokines would be increased in order to compensate for the loss of KC signaling in the knockout mice. However, the expression of both LIX and MIP-2 were no different in the joints of $\mathrm{KC}^{-/-}$mice when compared to their expression in the joints of WT mice (fig. 3.5 B).

Overall, these data suggest that, even with drastically reduced neutrophil and macrophage recruitment to sites of infection, mice were able to mount a successful immune response. The fact that cytokine and chemokine expression was unchanged in the $\mathrm{KC}^{-/-}$mice may help to explain how these mice were able to clear infection despite the reduced numbers of macrophages and neutrophils in the joints.

\section{F. KC mediates Lyme carditis severity.}

Besides arthritis, carditis is a significant sequelae that results from $B b$ infection in humans that is recapitulated by experimental murine Lyme borreliosis (126). While the results from Brown, et al. and this study proved the KC/CXCR2 axis mediated the development of Lyme arthritis, mechanisms that modulate the development of murine Lyme carditis have not yet been elucidated. Therefore, we extracted and sagittally bisected the hearts of $B b$-infected mice at days 11 and 21 post-infection and assessed them histologically for carditis severity and 
carditis type. Carditis severity and type scores were assigned similarly to arthritis severity scores.

On day 11 post-infection, neither WT nor $\mathrm{KC}^{-/-}$had developed carditis, reflecting delayed dissemination of $B b$ to the heart $(125,178)$ (fig. 3.6 A). However, on day 21 post-infection, $\mathrm{KC}^{--}$mice had significantly reduced carditis severity (fig. 3.6 A) and type scores (fig. 3.6 B) compared to WT mice indicating that $\mathrm{KC}$ signaling plays a role in the development of murine Lyme carditis.

\section{G. Neutrophil and macrophage infiltration into heart tissue is altered in the absence of KC.}

It is suggested that macrophages mediate the development and severity of Lyme carditis, as they are the dominant cell type present in cardiac lesions $(126,135,179)$. In an effort to assess the effect of KC deficiency on immune cell infiltration into the heart, we used immunohistochemical staining of sagittally bisected heart sections from WT or $\mathrm{KC}^{-/-}$mice on days 11 and 21 post- $\mathrm{Bb}$ infection. We used F4/80 and RB6-8C5 to label macrophages and neutrophils, respectively. Reflecting the results obtained using carditis severity and type scores (fig. 3.6), at day 11 post-infection, few immune cells were present in the hearts of WT or $\mathrm{KC}^{-/-}$mice. By day 21 post-infection, WT hearts exhibited severe carditis that coincided with the presence of a large number of both macrophages and neutrophils (fig. 3.7). $\mathrm{KC}^{-/-}$mice, however, displayed almost an almost complete absence of neutrophils in cardiac tissue, accompanied by a drastic reduction in the number of macrophages compared to WT mice at this time point 


\section{A}

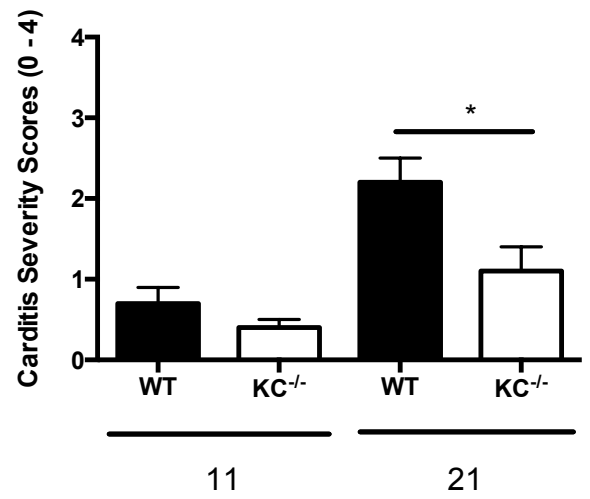

B

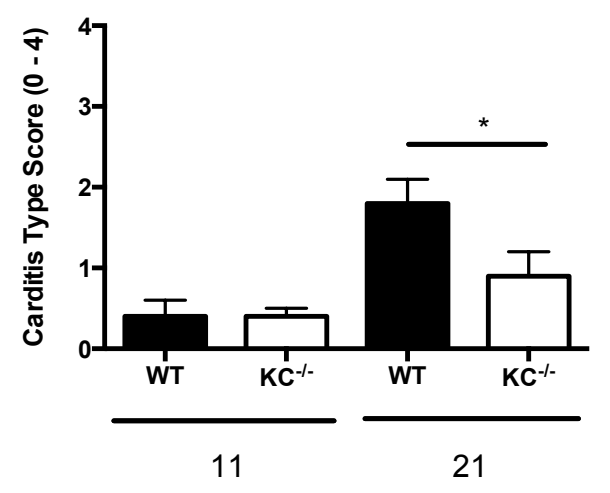

Fig. 3.6. $\mathrm{KC}^{-/-}$mice have attenuated Lyme carditis and altered immune cell infiltration into $B b$-infected heart tissue (134). Sagittally bisected heart sections from $8 \mathrm{WT}$ and $8 \mathrm{KC}^{-/-}$mice at days 11 and 21 post-infection were stained with H\&E and carditis severity (A) and type scores (B) were generated on a scale of $0-4$. Data shown are means \pm SEM, and * indicates a significant difference from WT at the same time point $(p<0.05)$. Assessment of histological sections and determination of carditis severity and type scores was performed by Jennifer Hughes-Hanks. 


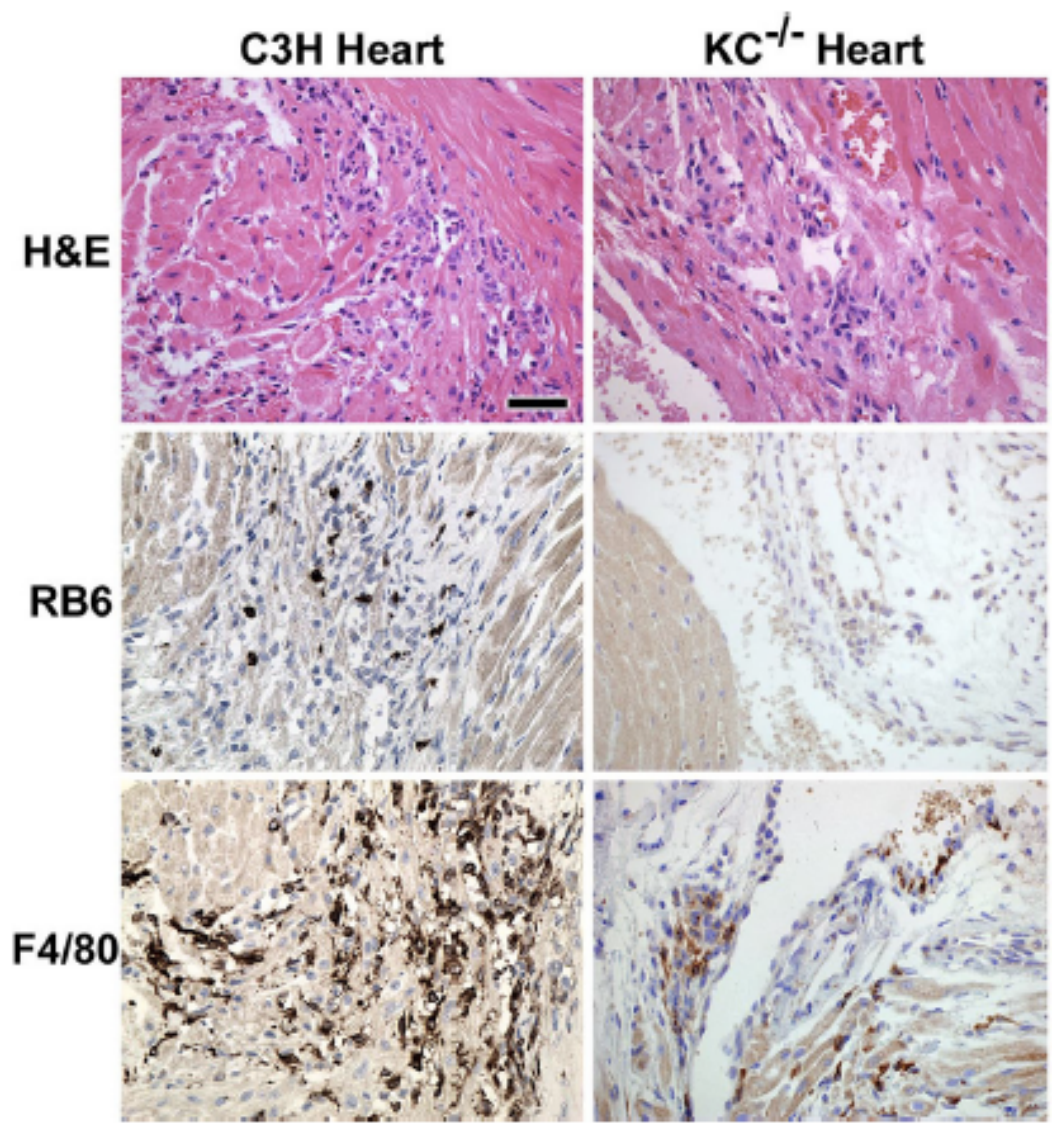

Fig. 3.7. $\mathrm{KC}^{-/-}$mice have significantly reduced numbers of neutrophils and macrophages in $B b$-infected heart tissue (134). Sagittally bisected heart sections were stained with the neutrophil marker RB6-8C5 (RB6) or the macrophage marker F4/80. Representative sections from day 21 post-infection are displayed above. Magnification X100. Bar $=50 \mu \mathrm{m}$. Determination of heart pathology and cell infiltrate was conducted by Jennifer Hughes-Hanks. 
(fig. 3.7). This data indicated that KC mediated the recruitment of neutrophils and macrophages to $B b$-infected cardiac tissue and was critical for the development of carditis.

\section{$\mathrm{H} . \mathrm{Bb}$ clearance from heart tissue is intact in the absence of $\mathrm{KC}$.}

Neutrophils and macrophages are the main phagocytic cells responsible for the clearance of $B b$ from infected tissues $(169-171,179,180)$. As the presence of these cells in the infected cardiac tissue of $\mathrm{KC}^{-/}$mice was greatly reduced (fig. 3.7), we hypothesized that the capacity to clear $B b$ from these tissues would be decreased. Therefore, we used quantitative Real-Time PCR to assess the presence of $B b$ in the hearts of WT and $\mathrm{KC}^{--}$mice and days 10,21 , and 35 post-infection. We found no differences in the quantity of $B b$ at any time point (fig. 3.8), indicating that although the numbers of neutrophils and macrophages in the hearts of $\mathrm{KC}^{-/-}$were reduced, this did not affect the ability of the mice to clear the infection.

\section{Heart cytokine and chemokine production is unaffected by the absence of} KC.

Because neutrophil and macrophage numbers were decreased in the hearts of $\mathrm{KC}^{-/-}$mice concurrent with reduced carditis severity, we were curious as to how the cytokine milieu in the hearts of these mice would be affected. Therefore, we extracted protein from the hearts of WT and $\mathrm{KC}^{-/-}$mice 11 and 21 


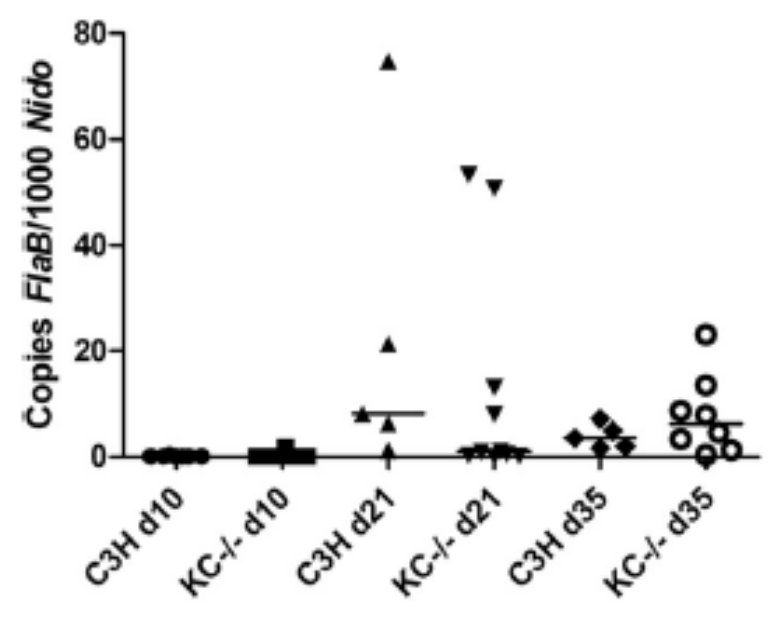

Fig. 3.8. $\mathrm{KC}^{-/-}$mice are not defective in their ability to clear $B b$ infection from cardiac tissue (134). Hearts were removed from mice at days 10, 21, and 35 post-infection. Spirochete DNA was detected in sagittally bisected heart tissue using quantitative Real Time PCR. Copies of the Bb flagellin gene (FlaB) were normalized to 1000 copies of the mouse gene nidogen (Nido). Data are representative of two separate experiments and bars indicate median values. 
days post- $B b$ infection and assessed the production of pertinent cytokines via ELISA.

TNF- $\alpha$, IL-1 $\beta$, and IL- 6 are all cytokines that are known to play important roles in the induction of inflammation. As neutrophil numbers, macrophage numbers, and cardiac inflammation were reduced in the $\mathrm{KC}^{-/-}$mice, we postulated that the production of these cytokines would be reduced, as well. However, the expression of TNF- $\alpha$, IL-1 $1 \beta$, and IL- 6 was no different in the hearts of $\mathrm{KC}^{-/-}$mice compared to WT mice (fig. $3.9 \mathrm{~A}$ ), and indicated that these cytokines were not affected by the absence of $\mathrm{KC}$ signaling and were perhaps produced by cells other than $\mathrm{F} 4 / 80^{+}$macrophages or $\mathrm{Gr}-1^{+}$neutrophils. Notably, IL-6 was produced in 10-fold higher quantities in heart tissue than joint tissue (fig. 3.5 A) suggesting that this cytokine is regulated differentially based on tissue microenvironment.

LIX and MIP-2, like KC, are CXCR2 ligands important for inducing the chemotaxis of neutrophils and macrophages to sites of infection. Because they share a common receptor and function similar to $\mathrm{KC}$, it was possible that LIX and MIP-2 would be increased in an effort to compensate for the loss of $\mathrm{KC}$ in the $\mathrm{KC}^{-}$ I- mice. However, the expression of these chemokines in the hearts of $\mathrm{Bb}$ infected mice at days 11 and 21 post-infection was unaffected by the loss of KC, as WT and $\mathrm{KC}^{-/-}$mice had equivalent levels of both LIX and MIP-2 at both time points (fig. 3.9 B). This data indicated that LIX and MIP-2 were not upregulated in an effort to compensate for the loss of $\mathrm{KC}$ signaling in the $\mathrm{KC}^{-/-}$mice, indicating that KC signaling through CXCR2 was critical for the recruitment of neutrophils 

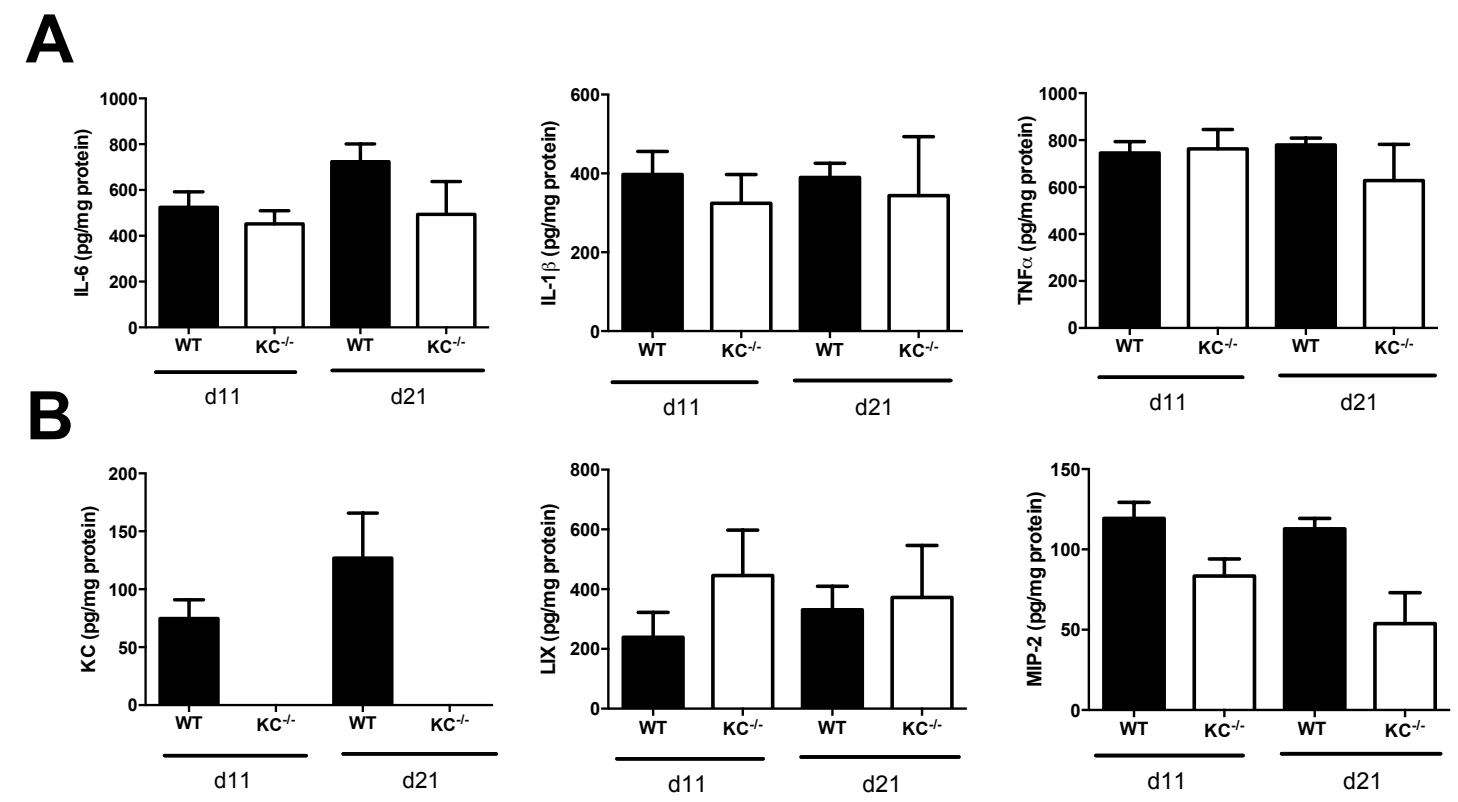

Fig. 3.9. Cytokine and chemokine levels in heart tissue of $\mathrm{KC}^{-/-}$mice are unaltered (134). Hearts were removed on days 11 and 21 post-infection and protein was extracted. Levels of the cytokines IL-6, IL-1 $\beta$, and TNF- $\alpha(\mathbf{A})$ and the chemokines KC, LIX, and MIP-2 (B) were quantified using ELISA. No significant differences were noted between $\mathrm{KC}^{-/-}$mice and WT mice at any time point. 
and macrophages to both joint and heart tissue during $B b$ infection. Interestingly, similar to what was seen with IL-6, it appears that differential tissue factors regulate the expression of LIX and MIP-2, as LIX was found in 10-fold higher quantities in the hearts of $B b$-infected mice, while MIP-2 was found in 10-fold higher quantities in joint tissue.

\section{DISCUSSION}

Neutrophils are known to play a significant role in the development of various models of arthritis $(109,161-166)$, as well as human Rheumatoid arthritis (RA) $(167,168)$, making neutrophil chemotactic factors attractive therapeutic targets (181-185). However, strategies targeting chemotactic factors in a therapeutic manner have had limited success, as the mechanisms that mediate the recruitment of neutrophils to sites of inflammation are still unclear.

Lyme arthritis is a valuable model for the study of various arthritides including RA as it allows for the study of multiple phases of an arthritic immune response (induction, peak, and resolution) whereas many autoimmune (chronic) models only allow the study of the induction and peak. This is valuable in terms of therapeutic design, as it allows for the study of resolution mechanisms that have the potential to be induced, rather than simply dampening inflammation, as most current therapeutics do.

However, the immunological mechanisms regulating the development and severity of experimental Lyme disease are incompletely understood. It was discovered early on that a genetic component is involved, as certain mouse 
strains were susceptible to inflammation resulting from infection with $B b$ [(C3H/He, SWR, CB.17 (SCID)] while other strains appeared resistant (C57BL/6, BALB/c, SJL) independent of their susceptibility to infection $(130,131)$. In 2003, Brown, et al. determined that the chemokines KC and MCP-1 were upregulated in genetically susceptible $\mathrm{C} 3 \mathrm{H} / \mathrm{HeJ}$ mice compared to resistant $\mathrm{C} 57 \mathrm{BL} / 6$ mice and that while the absence of CCR2, the receptor for MCP-1, had no effect on disease severity, the absence of CXCR2, the receptor for $\mathrm{KC}$, resulted in attenuated disease in susceptible $\mathrm{C} 3 \mathrm{H} / \mathrm{HeJ}$ mice. This was the first study to definitively determine a difference in immunological mechanisms regulating disease susceptibility and proved, for the first time, that Lyme arthritis was mediated by the recruitment of neutrophils. In the current study, we determined that the CXCR2 ligand KC was critical for the recruitment of neutrophils and in its absence the functionally similar CXCR2 ligands LIX and MIP-2 were unable to compensate for its loss. Thus, KC signaling through CXCR2 alone mediated the recruitment of neutrophils to infected tissues and subsequent development of experimental Lyme arthritis.

Surprisingly, we determined that the KC/CXCR2 axis not only regulated the development of experimental Lyme arthritis, but carditis as well. Neutrophils predominate during Lyme arthritis (130) while macrophages are the dominant cell type found in cardiac lesions $(126,135,179)$. As $K C$ is a potent neutrophil chemoattractant, we hypothesized that, in its absence, neutrophils would be reduced in arthritic joint tissue and thus disease severity would be reduced. However, carditis in the $\mathrm{KC}^{-/-}$mice was also significantly reduced and this 
reduction in severity was accompanied by an almost complete absence of neutrophils and a drastic reduction in macrophages in infected cardiac tissue. This information suggests that, although macrophages are the dominant cell type in cardiac tissue during Lyme carditis, neutrophils mediate the overall inflammatory response. The mechanisms by which neutrophils mediate carditis are still unclear.

Interestingly, although numbers of neutrophils and macrophages were drastically reduced in joint and heart tissue, the ability to clear $B b$ was unaffected in the absence of KC. In vitro, both neutrophils and macrophages are efficient at the phagocytosis and killing of $B b(179,186,187)$; however, the role of these cells in vivo is unclear. It has been shown that susceptibility to the development of Lyme arthritis or carditis is independent of susceptibility to infection, as both susceptible and resistant strains harbor similar amounts of $B b$ (130). The disparity between the development of disease and the ability to clear $B b$ infection remains unclear. However, it has been suggested that current technologies are outdated and that the qPCR primers used to detect the presence of $B b$ in infected tissues are unable to distinguish between live and dead spirochetes. If this is the case, it could be that although WT mice appear to have similar quantities of $B b$ in tissues compared to $\mathrm{KC}^{-/-}$mice, the DNA that is being detected is from killed $B b$, indicating that there is indeed a defect in the ability of $\mathrm{KC}^{-/}$to clear infection. However, this possibility is purely speculation.

Many of the hypotheses developed in an effort to explain the failure of current therapeutics targeting chemokines are centered on the fact that the 
chemokine system is highly redundant. In 2003, Brown et al. discovered that in the absence of CXCR2 signaling, neutrophil recruitment was diminished and Lyme arthritis severity was attenuated. Three chemokines, perhaps redundantly, signal through this receptor: LIX; MIP-2; and KC. As KC was expressed more highly in susceptible mouse strains when compared to resistant mouse strains and $\mathrm{KC}^{-/-}$mice exhibited similar defects in neutrophil recruitment and arthritis severity, it is highly likely that KC signaling through CXCR2 mediates these effects. Due to the possibly redundant nature of KC, LIX, and MIP-2, we hypothesized that the expression of LIX and MIP-2 might be increased in an effort to compensate for the loss of $\mathrm{KC}$ in $\mathrm{KC}^{-/-}$mice. However, this did not appear to be the case, as LIX and MIP-2 were expressed at similar levels in the $\mathrm{KC}^{-/-}$mice as WT mice. The fact that neutrophil recruitment was drastically reduced in the absence of KC suggests that, in the case of Lyme arthritis and carditis, LIX, MIP-2, and $\mathrm{KC}$ are not redundant in nature and that $\mathrm{KC}$ alone mediated the recruitment of neutrophils to sites of $B b$ infection via signaling through CXCR2.

Due to the fact that $\mathrm{KC}^{-/-}$mice exhibited fewer neutrophils and macrophages in infected hearts and joints, we hypothesized that the expression of cytokines would also be reduced in these mice. We therefore looked at the expression of three cytokines known to be important in developing immune responses, especially arthritis (188-191), and found no differences in the expression of IL- 6 , TNF- $\alpha$, or IL-1 $1 \beta$ at any time point. These data suggest that neutrophils and macrophages were not the primary producers of these proteins 
in joint and heart tissue and that these cytokines were not directly linked the development of Lyme arthritis or carditis. These data may also suggest that although $\mathrm{KC}$ is important for the recruitment of neutrophils, it does not affect the activation of circulating neutrophils. However, as these cytokines are important for the development of an immune response, the fact that their expression is unaltered may help to explain why these mice are able to effectively clear infection.

Prior to this study, the mechanisms regulating the development of Lyme arthritis and carditis were incompletely understood. In combination with results obtained by Brown, et al., it is now well understood that the development of both Lyme arthritis and carditis is driven by neutrophil recruitment mediated by the $\mathrm{KC} / \mathrm{CXCR} 2$ axis. 


\section{CHAPTER FOUR}

\section{THE EFFECT OF CYCLOOXYGENASE-2 DEFICIENCY ON THE CYTOKINE/CHEMOKINE/LIPID MILIEU DURING LYME ARTHRITIS}

\section{INTRODUCTION}

Cyclooxygenase-2 (COX-2) has long been associated with inflammation as its expression is inducible and it is upregulated in immune cells during inflammation $(25-27,52,53)$. In 2002, Anguita, et al. reported that COX-2 mRNA expression correlated with the degree of arthritis severity associated with $B b$ induced Lyme arthritis and that specific inhibition of this enzyme resulted in reduced disease severity (140). However, in a similar experiment, Blaho, et al. found that specific inhibition of COX-2 (using Celecoxib-containing chow) in $\mathrm{Bb}$ infected mice did not result in the alleviation of arthritis severity (141). At day 17 post-infection, the peak of disease severity, mice fed control chow and mice fed Celecoxib-containing chow had similar disease severity. Interestingly, on day 35 post-infection, a time point at which control chow-fed mice had resolved arthritis, the joints of mice fed Celecoxib-containing chow remained severely inflamed (141). Bb-specific antibody production ( $\lg M$ and $\lg G$ ) was intact in these mice and they were able to clear $B b$ from tissues as effectively as control chow-fed mice, suggesting that the defect in arthritis resolution was not due to an inability to mount an effective immune response and clear bacteria from tissue. Results were similar whether mice were fed the Celecoxib-containing chow throughout 
the entire course of infection or whether the chow was only fed to day 14 postinfection indicating that early COX-2 metabolite production played an important role in the development of resolution mechanisms. These results are consistent with the hypothesis presented by Drs. Charles N. Serhan and Bruce D. Levy suggesting that lipid mediators produced early during inflammation are responsible for programming resolution mechanisms $(7,82)$.

To determine the resolution mechanisms rendered defective by COX-2 inhibition, we conducted a similar time course study in which mice were fed either normal mouse chow or Celecoxib-containing chow for two weeks prior to infection with $B b$ and the production of eicosanoids, cytokines, and chemokines was assessed. Furthermore, we conducted three studies in which mice were treated with either prostaglandin $J_{2}\left(P G J_{2}\right), P G E_{2}$, or an agonist to the prostaglandin E receptor 2 (EP2) to determine whether defective production of these metabolites was responsible for the lack of resolution seen during COX-2 inhibition.

\section{RESULTS}

\section{A. Inhibition of COX-2 results in attenuated ankle joint swelling, but has little effect on arthritis severity.}

Mice ( $n=30$ per group) were fed a chow supplemented with 1000 ppm Celecoxib (equivalent to $\sim 2 \mathrm{mg} / \mathrm{kg}(141)$ ) or a normal chow diet (control mice) for two weeks prior to infection with $1 \times 10^{5} \mathrm{Bb}$. Tibiotarsal (ankle) joint swelling was monitored throughout the course of disease and on days $0,7,14,24$, and 62 
post-infection, mice were sacrificed and ankle joint tissues were collected for the analysis of arthritis severity, arthritis type scores, and tissue eicosanoid production. Celecoxib-fed mice had significantly reduced joint swelling on days 24, 34, 46, and 62 post-infection (fig. 4.1 A), indicating that metabolites produced via the COX-2 pathway were responsible for ankle joint swelling associated with Lyme arthritis. Typically, joint swelling is indicative of underlying tissue inflammation; however, this is not always the case during experimental Lyme arthritis (153). Although ankle swelling was reduced at most time points, arthritis severity was similar in Celecoxib-fed and control mice at all time points except for day 24 post-infection (fig. 4.1 B). By day 62 post-infection, both Celecoxib-fed and control mice had fully resolved arthritis.

Arthritis type scores in this experiment were highly variable and thus difficult to analyze; however, the peak of arthritis severity in both Celecoxib-fed and control mice (days 14 - 24 post-infection) was characterized by a dominating presence of neutrophils at days 7,14 , and 24 post-infection, while the beginning (day $0-7$ post-infection) and end (day 62 post-infection) of the immune response was characterized by an influx of macrophages (fig. 4.1 C). Celecoxibfed mice exhibited no significant difference in infiltrating immune cells compared to control mice, suggesting that COX-2 inhibition does not directly affect immune cell recruitment during Lyme arthritis. 
A

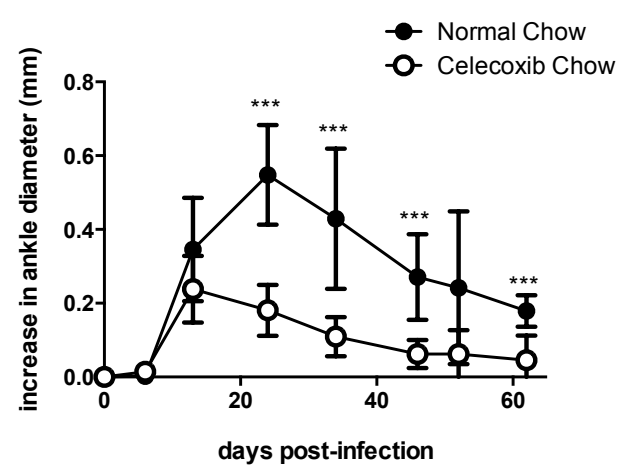

C

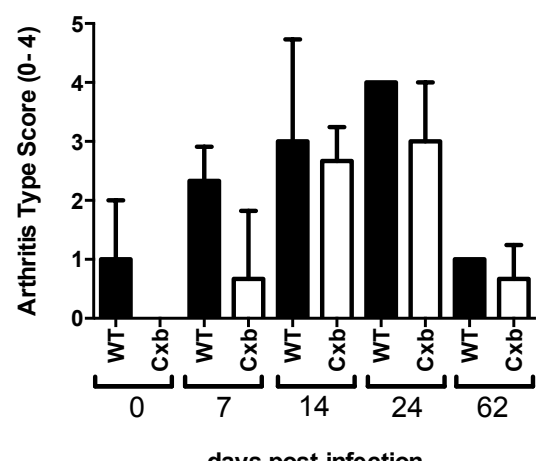

B

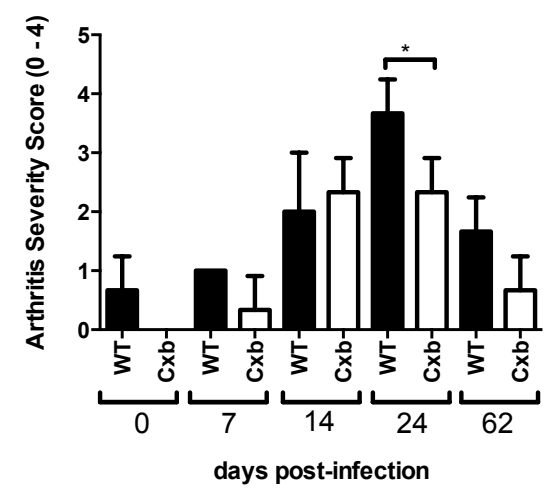

Fig. 4.1. Progression of arthritis in Celecoxib-fed mice. 30 mice were fed a chow supplemented with 1000 ppm Celecoxib and 30 mice were fed a normal chow diet as controls. Mice were infected with $1 \times 10^{5} \mathrm{Bb}$ and disease was allowed to progress for 62 days. Throughout the course of disease, tibiotarsal joint (ankle) swelling was monitored using calipers (A). On days $0,7,14,24$, and 62 post-infection, 6 control and 6 Celecoxib-fed mice were sacrificed. Arthritis severity (B) and type (C) was determined using histological samples as described in Methods \& Materials. Jennifer Hughes-Hanks performed histological assessment of pathology. ${ }^{* * *}=P<0.001$. 


\section{B. Inhibition of COX-2 affects the production of numerous eicosanoids.}

To determine the effects of COX-2 inhibition on global eicosanoid production, we isolated lipids from ankle joint tissue of control and Celecoxib-fed mice on days $0,7,14,24$, and 62 post-infection. Isolated lipid samples were sent to Dr. Darren S. Dumlao in Dr. Edward A. Dennis' lab at the University of California - San Diego for analysis via LC-MS/MS. Fold increases or decreases in metabolite expression were compared and differences are shown on a heat map (fig. 4.2). As expected, the expression of the majority of COX-derived metabolites were decreased in the Celecoxib-fed mice (e.g.: PGF ${ }_{1 \alpha}, P G_{2 \alpha}$, $\mathrm{PGE}_{2}, \mathrm{PGD}_{2}$ ). Largely, lipoxygenase (LOX) metabolites were unaffected, 15LOX metabolites exhibited mild reductions in expression (15-HETE), and 12-LOX

metabolites exhibited both increases (9-HODE) and decreases (12-HETE) in expression. Metabolites generated via the cytochrome P450 (CYP) pathway, including 18-HEPE, 17,18-EpETE, 9.10-EpOME, and 12,13-EpOME were generally increased in Celecoxib-fed mice. These results indicate that COX-2 inhibition dynamically affects the production of several eicosanoids and that these effects are not limited to the COX-2 pathway.

\section{COX-2 metabolites are reduced in mice fed Celecoxib-containing chow.}

The LC-MS/MS-generated heat map indicated reductions in the COX-2 metabolites $\mathrm{PGE}_{2}, 6 \mathrm{k}-\mathrm{PGF}_{1 \alpha}$, and 11-HETE upon COX-2 inhibition (fig. 4.2). To further analyze these reductions, we calculated the amounts of these eicosanoids in picograms per milligram of joint tissue (fig. 4.3). 


\section{$\begin{array}{llllll}\text { DAY } & 0 & 7 & 14 & 24 & 62\end{array}$}

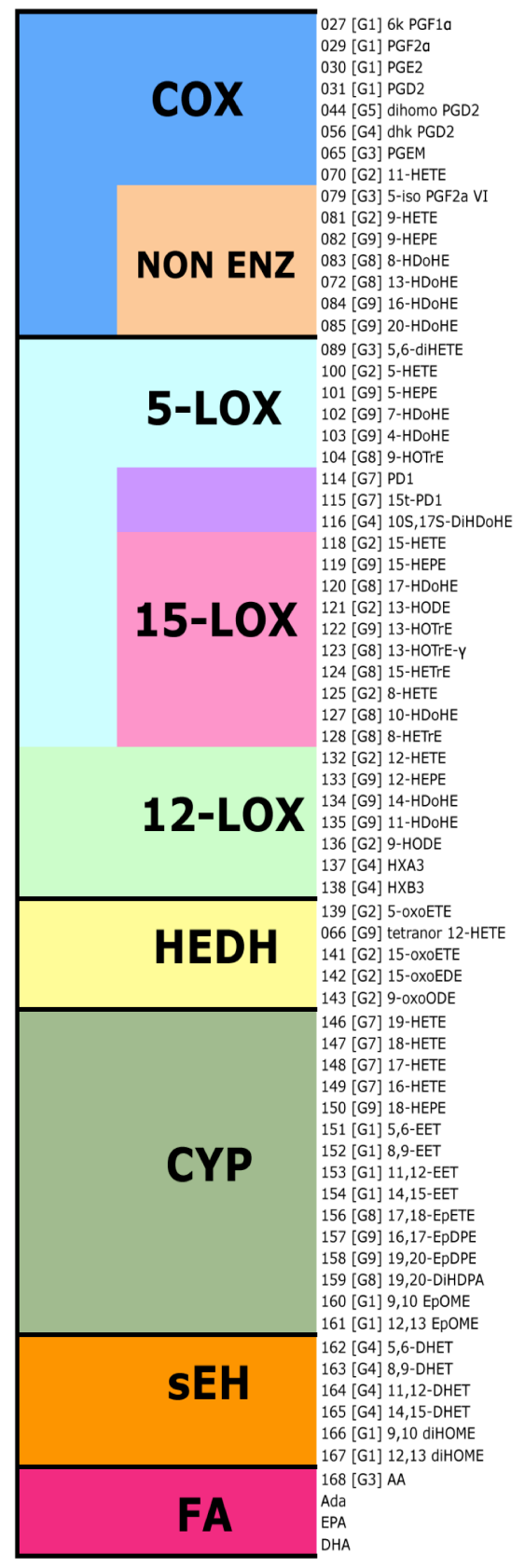
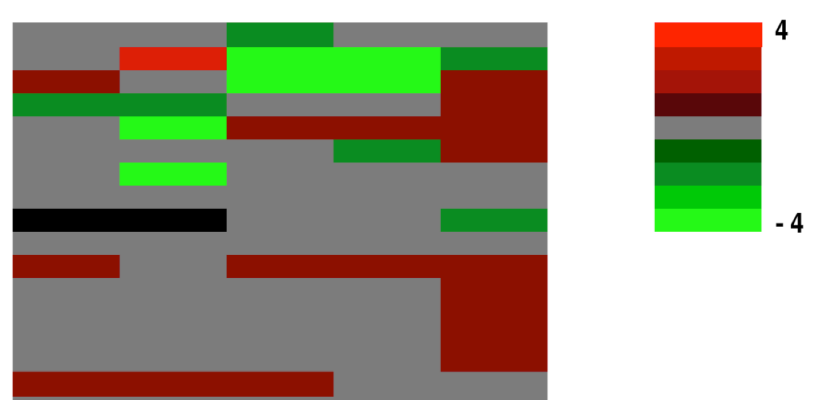

$-4$

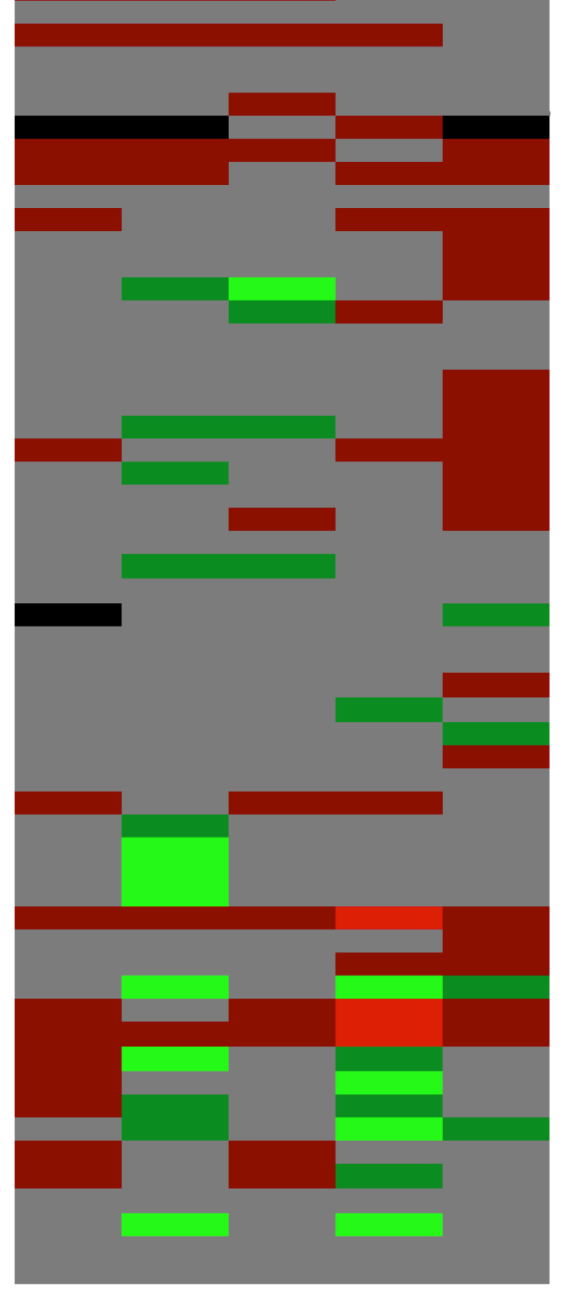


Fig. 4.2. Global eicosanoid production in Celecoxib-fed mice. Lipids were extracted from ankle joint tissue of mice fed either a normal chow diet (control) or a diet supplemented with Celecoxib on days $0,7,14,24$, and 62 post-infection with $\mathrm{Bb}$. Eicosanoids were quantified via LC-MS/MS. Values shown represent fold change in metabolite production in the Celecoxib-fed mice compared to control mice at the same time point. A red color indicates a fold increase in metabolite production and a green color indicates a fold decrease in metabolite production. (LC-MS/MS analysis and construction of heat map conducted by Dr. Darren S. Dumlao, UCSD.) 
Although the heat map indicated that $\mathrm{PGE}_{2}$ was reduced four-fold in the Celecoxib-fed mice on days 14 and 24 post-infection (fig. 4.2), this reduction did not reach statistical significance (fig. 4.3 A). However, the reduced $\mathrm{PGE}_{2}$ expression at these time points may explain the reduced swelling in the Celecoxib-fed mice, as $\mathrm{PGE}_{2}$ is a known mediator of swelling due to its effects on vascular permeability and edema formation (192). $6 k-P G F_{1 \alpha}$ is a stable breakdown product of the COX-1 metabolite $\mathrm{PGI}_{2}$, which serves as a potent vasodilator (193). Production of this metabolite was reduced two-fold (fig. 4.2), but once again this reduction did not reach statistical significance (fig. 4.3 B). 11-HETE is implicated to be anti-inflammatory and was significantly reduced at day 7 post-infection in the Celecoxib-fed mice compared to control mice (fig. 4.2 C). The reduction in this metabolite may suggest a mechanism by which COX-2 inhibition prolongs the inflammatory state of arthritic mice (141).

\section{Lipoxygenase (LOX) metabolites are differentially affected by COX-2 inhibition.}

COX-2 inhibition had differential effects on metabolites production via the three lipoxygenase pathways. 5-HETE, a metabolite produced via the 5-LOX pathway, was unaffected by COX-2 inhibition (fig. 4.4 A). However, 15-HETE, produced via the 15-LOX pathway, and 12-HETE and $\mathrm{HXB}_{3}$, produced via the 12-LOX pathway, were all significantly reduced on day 7 post-infection (fig. 4.4 B - D). 
A

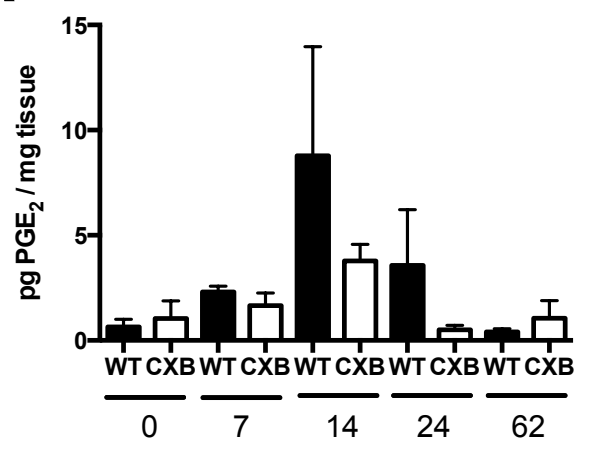

C

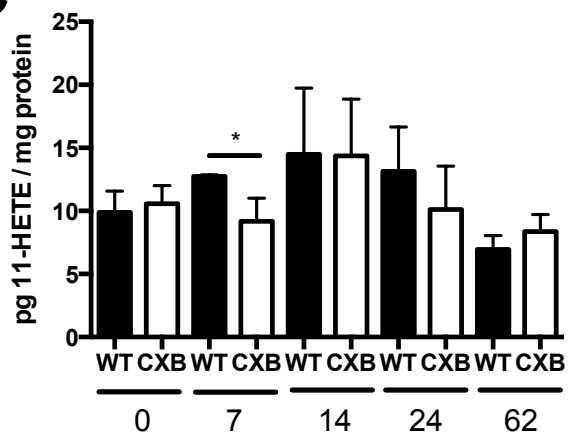

B

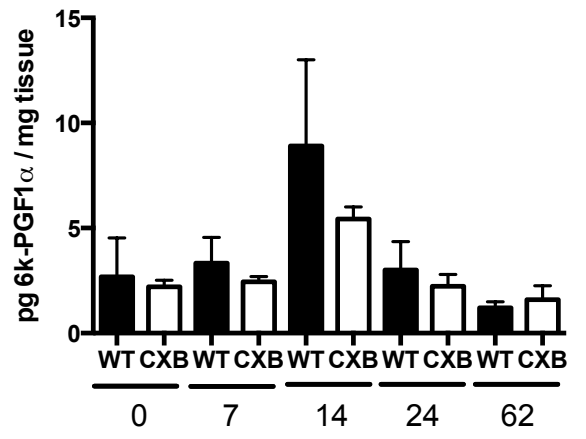

Fig. 4.3. Production of cyclooxygenase-derived metabolites in the ankle joints of Celecoxib-fed mice. Lipids were extracted from the ankle joints of control and Celecoxib-fed mice on days $0,7,14,24$, and 62 post-infection with Bb. Quantities of $\mathrm{PGE}_{2}(\mathbf{A}), 6 \mathrm{k}-\mathrm{PGF}_{1 \alpha}(\mathbf{B})$, and 11-HETE (C) were detected using LC-MS/MS. $\quad{ }^{*}=P<0.05 . \quad$ (LC-MS/MS performed my Dr. Darren $S$. Dumlao.) 
A

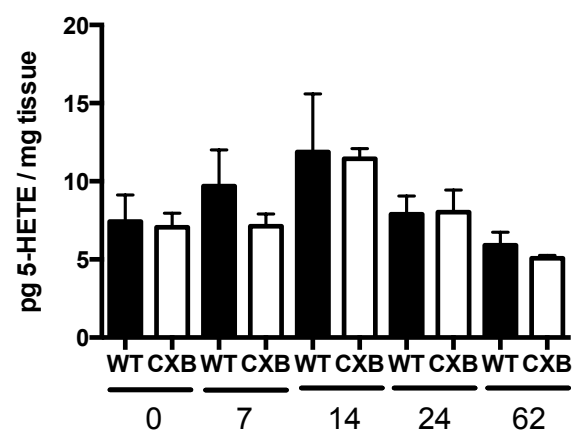

C

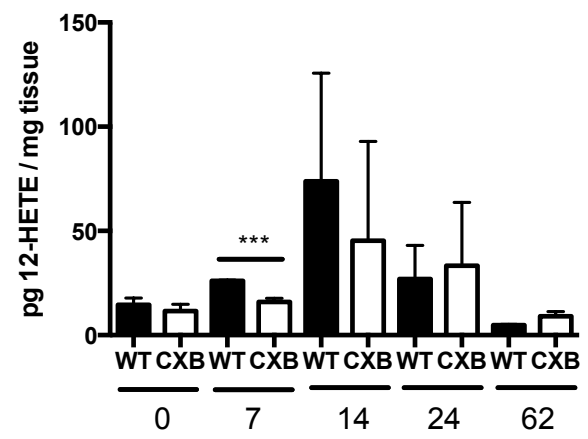

B

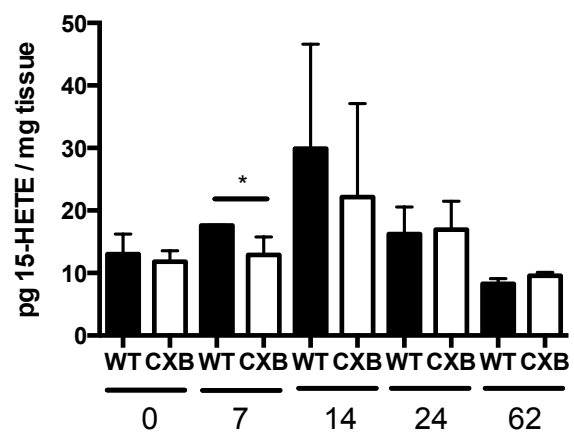

D

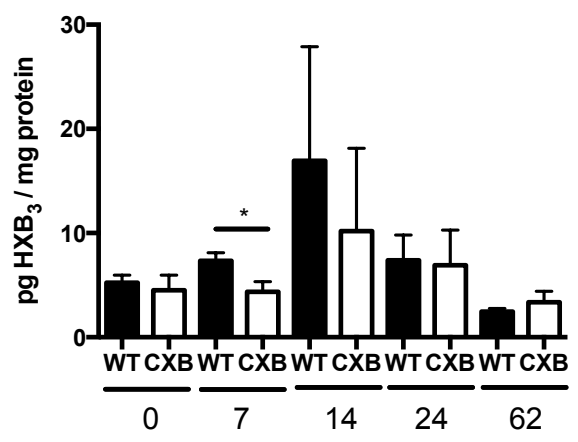

Fig. 4.4. Production of lipoxygenase metabolites in the ankle joints of Celecoxib-fed mice. 30 mice were fed a normal chow diet and 30 mice were fed a diet supplemented with Celecoxib. Mice were infected with $B b$ and disease was allowed to progress for 62 days. On days $0,7,14$, and 24 post-infection, lipids were extracted from ankle joint tissue of mice and the lipoxygenase metabolites 5-HETE (A), 15-HETE (B), 12-HETE (C), and $\mathrm{HXB}_{3}$ were detected using LC-MS/MS. ${ }^{*}=P<0.05 .{ }^{* * *}=P<0.001 . \quad$ (LC-MS/MS analysis was conducted by Dr. Darren S. Dumlao.) 
As both the 12- and 15-LOX pathways are involved in pro-resolving lipoxin production and COX-2 inhibition appeared to negatively impact the production of metabolites produced via these pathways, it is possible that COX-2 inhibition interferes with pro-resolution pathway. This could potentially explain the defective resolution of arthritis in Celecoxib-treated mice observed by Blaho, et al. (141).

The fact that the eicosanoid data were so variable and often did not reach statistical significance is likely due to a low sample size. Only three joints were available for eicosanoid analysis per time point. A repeat of this experiment with a larger sample size is needed in order to better ascertain the effects of COX-2 inhibition on eicosanoid production. A power analysis would be an effective tool for the determination of a sample size that would adequately provide useful results.

\section{E. Supplementation of $\mathrm{COX}-2^{-/-}$mice with $\mathrm{PGJ}_{2}$ does not restore the ability to resolve arthritis.}

Similar to the phenotype observed in Celecoxib-fed mice, Blaho, et al. observed that mice deficient in COX-2 $\left(\mathrm{COX}_{-} 2^{--}\right)$were unable to resolve Lyme arthritis (141). 15 deoxy- $\Delta 12,14$-prostaglandin $J_{2}\left(15 d-P G J_{2}\right)$ is a stable breakdown product of $\mathrm{PGD}_{2}$. It is reportedly anti-inflammatory as, in vitro, it binds the nuclear receptor PPARY and inhibits NF-KB activation (65-68). COX-2${ }^{1-}$ mice and mice treated with the COX-2 inhibitor Celecoxib have reduced levels of both $P G D_{2}$ and $15 d P G J_{2}((144)$ and fig. 4.2). Thus, we hypothesized that 
treatment of $\mathrm{COX}-2^{-/-}$mice with $15 \mathrm{~d}-\mathrm{PGJ}_{2}$ on days $3-14$ post-infection may restore the ability to resolve arthritis in these mice. Treatment of mice with $15 \mathrm{~d}$ $P G J_{2}$ on days 3-14 was decided based on the fact that COX-2 inhibition early during infection (day -1 through day 14 post-infection) resulted in inhibition of resolution, indicating that metabolites produced via this pathway early during inflammation were responsible for inducing subsequent resolution (141). We infected 10 wild type (WT) $\mathrm{C} 3 \mathrm{H} / \mathrm{HeJ}$ and $10 \mathrm{C} 3 \mathrm{H} \mathrm{COX}-2^{-/-}$with $1 \times 10^{5} \mathrm{Bb}$. Five WT and $5 \mathrm{COX}^{-{ }^{-1}}$ mice were subcutaneously treated with $50-\mu \mathrm{L}$ vehicle PBS

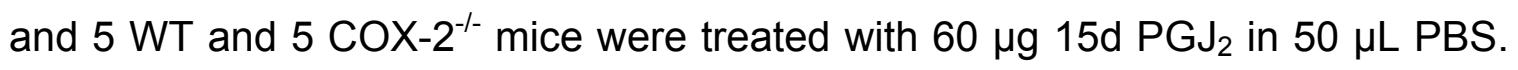
Disease was allowed to progress for 35 days and ankle swelling was monitored throughout the course of disease.

Although ankle swelling was slightly reduced in $\mathrm{COX}^{-2^{--}}$mice as compared to WT mice, this trend did not reach statistical significance (fig. 4.5 A). Furthermore, treatment of COX $-2^{-/-}$mice with $15 \mathrm{~d} \mathrm{PGJ}_{2}$ did not affect the ankle swelling in these mice compared to COX-2/-- mice treated with PBS (fig. 4.5 B). Although the data from this study suggest that $15 d P G J_{2}$ does not affect ankle swelling in $\mathrm{COX}_{-2} 2^{--}$mice, some major flaws in the experimental design of this study are apparent: 1) the goal of the study was to assess the affect of supplementation with $15 d-P G J_{2}$ on arthritis resolution, not ankle joint swelling. Thus, it would have been much more effective to conduct this study with time points at which arthritis severity was assessed rather than simply monitoring ankle swelling. 2) Ankle swelling is not always indicative of underlying inflammation. Although no effects were seen on the progression of ankle 

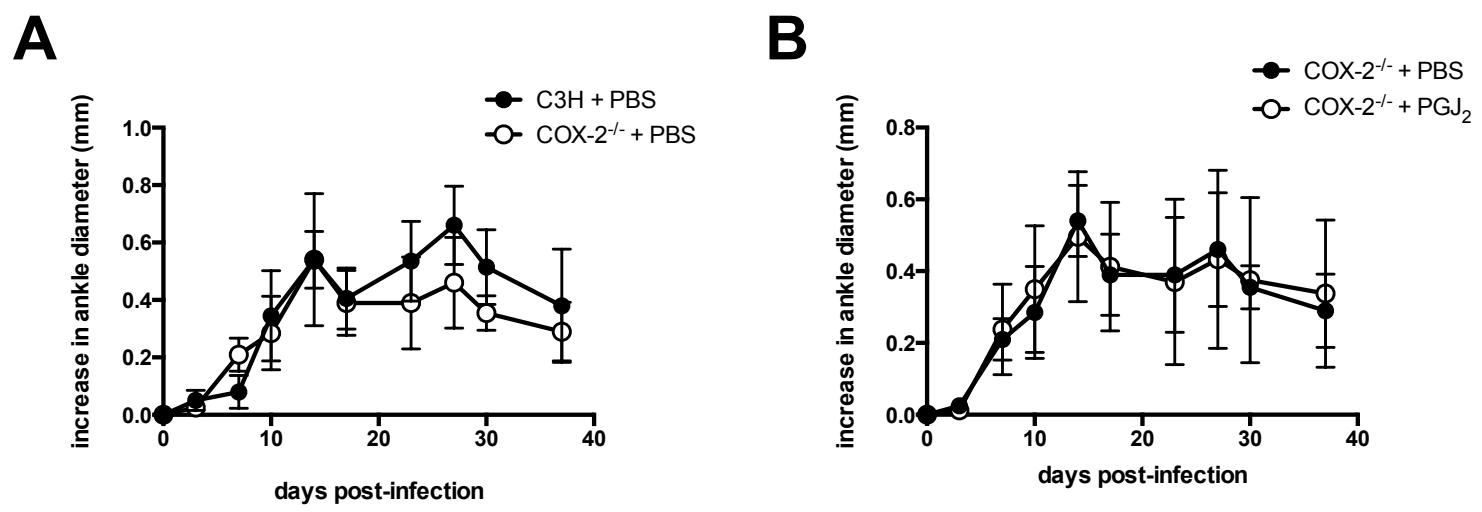

Fig. 4.5. $6 \mathrm{k}-\mathrm{PG} \mathrm{J}_{2}$ treatment of $\mathrm{COX}-2^{-/-}$mice. $10 \mathrm{WT}$ mice and $10 \mathrm{COX}-2^{-/-}$ mice were infected with $B b$. 5 WT and $5 \mathrm{COX}_{-} 2^{-/-}$mice were treated subcutaneously with PBS and 5 WT mice and $5 \mathrm{COX}-2^{--}$mice were treated with $60 \mu \mathrm{g} 15 \mathrm{~d}-\mathrm{PGJ}_{2}$ on days 3-14 post-infection. Ankle joint swelling was monitored using metric calipers for 35 days. 
swelling in these mice, the underlying immune response could have been affected. Once again, this would suggest that assessing arthritis severity via histology would have been more effective. 3) Eicosanoids are bioactive and insoluble in most vehicles (including PBS in most instances). Although PBS was originally selected as a vehicle due to its inert qualities, it is possible that $15 d-$ $P G J_{2}$ was unable to effectively solubilize in PBS. It is also possible that $15 d-$ $P G J_{2}$, due to its bioactive nature, degraded prior to being able to exert any biological effects. A more effective route may have been intravenous or intraperitoneal injection and a more effective vehicle may have been ethanol or methyl acetate (diluted in PBS).

\section{F. Supplementation of $\mathrm{COX}-2^{-/-}$mice with $\mathrm{PGE}_{2}$ does not restore the ability to resolve arthritis.}

$\mathrm{PGE}_{2}$ is believed to mediate the "switch" from pro-inflammatory eicosanoid production to anti-inflammatory eicosanoid production by reducing the expression of 5-LOX and $\mathrm{LTB}_{4}$ production and increasing 15-LOX expression and 15-HETE (and subsequent $\mathrm{LXA}_{4}$ ) production (82). As $\mathrm{PGE}_{2}$ production was reduced in Celecoxib-fed and $\mathrm{COX}-2^{-/-}$mice, we hypothesized that the absence of this eicosanoid might be responsible for defective resolution of Lyme arthritis in these mice. Therefore, we performed an add-back experiment to restore $\mathrm{PGE}_{2}$ levels in $\mathrm{COX}-2^{--}$mice infected with $B b$ and determined the effect that this had on arthritis resolution. Ten WT mice and $12 \mathrm{COX}-2^{--}$mice were infected with $1 \times$ $10^{5} \mathrm{Bb}$ and either treated with PBS (vehicle) or 1.0- $\mathrm{gg}$ of $\mathrm{PGE}_{2}$. Mice were 
treated via daily subcutaneous inoculations in the scruff of the neck throughout the course of infection. The infection was allowed to progress for 35 days, after which mice were sacrificed and ankle joint tissues were collected for the assessment of arthritis severity. We found that ankle joint arthritis severity in the $\mathrm{COX}-2^{-/-}$mice was similar to that of WT mice whether treated with $\mathrm{PGE}_{2}$ or not (fig. 4.6). It is worth noting, however, that in contrast to defective resolution seen previously in these $\mathrm{COX}-2^{-/-}$mice, the $\mathrm{COX}-2^{-/-}$mice treated with PBS had equivalent severity scores compared to WT mice treated with PBS at day 35 post-infection. This could indicate that the infection was not as effective as that completed by Blaho, et al. (as a result of different passages of $B b$, ineffective infection, etc). Furthermore, the same issues discussed in regards to the $15 d-$ $P G J_{2}$ treatment are present in this study, as well: the route of treatment (subcutaneous) and the vehicle (PBS) may not have been ideal of effective delivery of $\mathrm{PGE}_{2}$.

In order to better assess the effect of $\mathrm{PGE}_{2}$ on the resolution of Lyme arthritis, we decided to directly target specific $\mathrm{PGE}_{2}$ receptors. The EP2 and EP4 receptors are known to mediate many of the inflammatory effects of $\mathrm{PGE}_{2}$ (91, 194-197). In an effort to bypass the risk of $\mathrm{PGE}_{2}$ degrading prior to activating its receptor, we decided to utilize the stable EP2 agonist, Butaprost. Ten WT and 8 $\mathrm{COX}_{-2^{--}}$mice were infected with $1 \times 10^{5} \mathrm{Bb} .2 \mathrm{WT}$ were left untreated, 4 WT and $4 \mathrm{COX}_{-2} \mathrm{-}^{--}$mice were treated with vehicle (dilution of ethanol in PBS), and 4 WT

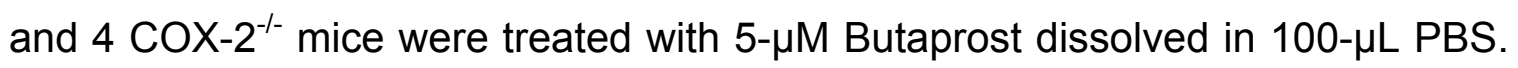
Treatments were delivered intraperitoneally on days $5-7$ post-infection and 


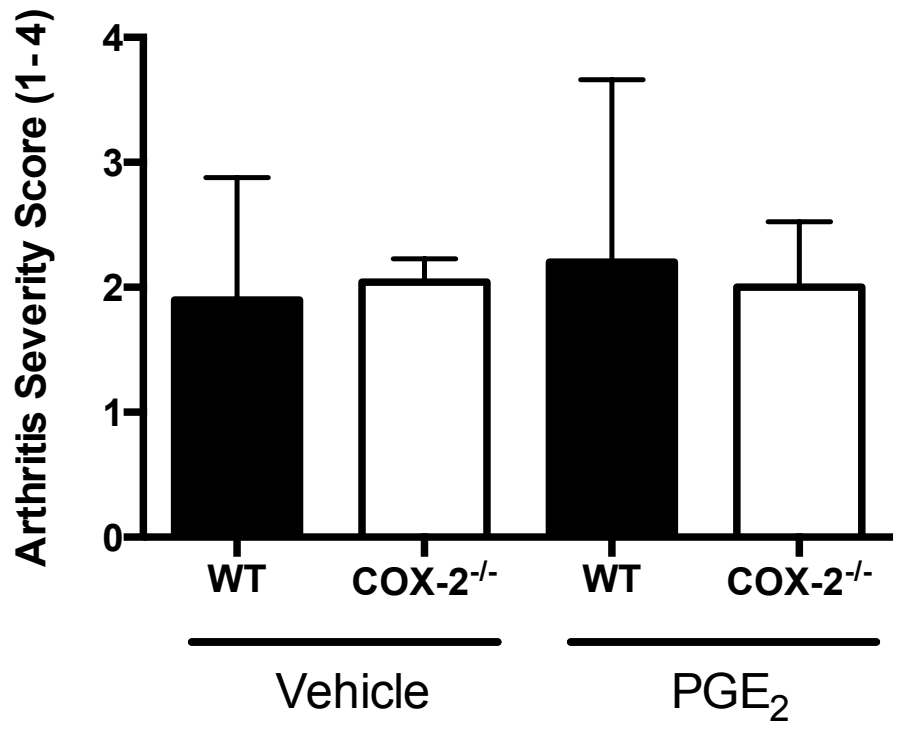

Fig. 4.6. Treatment of COX-2 ${ }^{-/-}$mice with $\mathrm{PGE}_{2 .}$ 10 WT and $12 \mathrm{COX}-2^{-/-}$mice were infected with $1 \times 10^{5} \mathrm{Bb}$. $5 \mathrm{WT}$ and $6 \mathrm{COX}-2^{-/-}$were treated subcutaneously with PBS and 5 WT and 6 COX-2-- mice were treated with $1.0-\mu \mathrm{g} \mathrm{PGE}_{2}$ daily throughout the course of infection. On day 34 post-infection, mice were sacrificed and ankle joints were collected, fixed, mounted, and stained using $\mathrm{H}$ \& E. Arthritis severity was assessed histologically by Jennifer Hughes-Hanks. 
days $12-15$ post-infection, the time points at which $\mathrm{PGE}_{2}$ expression peaks during $B b$ infection in WT mice (143). Disease was allowed to progress for 42 days, after which mice were sacrificed and tissues were collected for the histological assessment of joint tissue severity. As previously reported by Blaho, et al. (141), COX-2 ${ }^{-1-}$ mice treated with PBS had increased joint severity as compared to WT mice treated with PBS or left untreated, indicating that as of day 42 post-infection, COX-2-/- mice were unable to resolve Lyme arthritis (fig. 4.7). However, mice treated with Butaprost had arthritis that was comparable to WT mice, indicating that stimulation of the EP2 receptor in COX-2 ${ }^{-1-}$ mice enabled these mice to resolve Lyme arthritis. These data suggest that $\mathrm{PGE}_{2}$ signaling through the EP2 receptor mediates the resolution of Lyme arthritis and that inhibition of COX-2 disrupts this process. However, caution should be had when interpreting these results as this experiment has only been conducted successfully one time due to the limited availability of $\mathrm{COX}-2^{-1-}$ mice.

\section{G. Cytokine and chemokine production is reduced in COX-2 $2^{-/-}$mice.}

Although the lack of resolution of Lyme arthritis observed during COX-2 inhibition was likely due to the effects on the eicosanoid microenvironment, downstream effects on other immune pathways are likely playing a role, as well. As eicosanoids are known to be involved in the regulation of cytokine and chemokine production (often due to effects on NF-kB), we wanted to assess whether any pertinent cytokines or chemokines were affected during COX-2 inhibition. Therefore, 30 mice were fed a chow supplemented with $1000 \mathrm{ppm}$ 


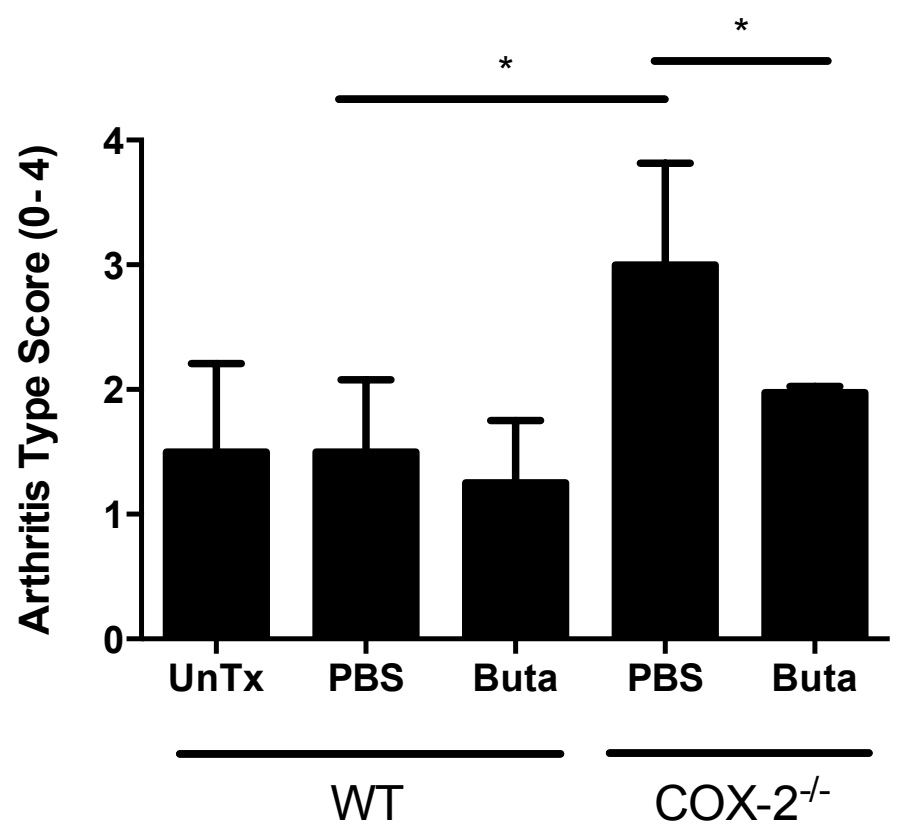

Fig. 4.7. Treatment of COX-2 ${ }^{-/-}$mice with the EP2 agonist Butaprost. 10 WT mice and $8 \mathrm{COX}-2^{-/-}$mice were infected with $1 \times 10^{5} \mathrm{Bb} .2 \mathrm{WT}$ mice were left untreated, 4 WT mice were treated with PBS, and 4 WT mice were treated with 5- $\mu \mathrm{M}$ Butaprost. $4 \mathrm{COX}-2^{-{ }_{-}}$mice were treated with PBS and $4 \mathrm{COX}-2^{-{ }^{-}}$mice were treated with $5-\mu \mathrm{M}$ Butaprost. Disease was allowed to progress for 42 days. On day 42 post-infection, ankle joint tissues were collected, fixed, mounted, and stained with $H$ \& E for histological assessment of arthritis severity. Histological assessment of pathology performed by Jennifer Hughes-Hanks. ${ }^{*}=P<0.05$. 
Celecoxib and 30 mice were fed a normal chow diet (control mice) for two weeks prior to infection with $1 \times 10^{5} \mathrm{Bb}$. On days $0,7,14,24$, and 62 post-infection, mice were sacrificed and tissues were collected for the quantification of TNFa, IL6, IL-10, and KC production via ELISA. All cytokines trended lower at all time points in the Celecoxib-fed mice compared to WT mice (fig. 4.8). TNFa and IL10 were significantly reduced at the peak of infection, day 24 post-infection (fig. 4.1 B, fig. 4.8 A). The effects of COX-2 metabolites on these two cytokines are often dependent on the concentration of metabolite present (or administered, in the case of in vitro experiments), but studies have shown that $\mathrm{PGE}_{2}$ is able to inhibit TNFa production and induce IL-10 production (198-201). As TNFa and IL10 were only affected mildly and only on day 24 post-infection, it could be that the mild reduction in $\mathrm{PGE}_{2}$ production observed in the Celecoxib-fed mice (fig. 4.3 A) was sufficient to have a negative impact on the production of these cytokines. The production of IL-6 is highly regulated by COX-2-derived metabolites (202-205) and its production was significantly reduced at every time point analyzed in Celecoxib-fed mice (fig. 4.8 B). KC, which is required for the development of Lyme arthritis (134), was significantly reduced on days 7 and 14 post-infection (fig. 4.8 D).

Similarly, we assessed the production of TNF $\alpha$, IL-12p70, IL-1 $\beta$, and KC in COX $-2^{-/-}$mice. In order to do this, we infected 4 WT and 4 COX $-2^{-/-}$mice with $1 \times$ $10^{5} \mathrm{Bb}$ and allowed disease to progress to day 24 post-infection (the peak of severity, fig. 4.1 B). Throughout the course of infection, ankle swelling was monitored. On day 24 post-infection, ankle joint tissue was extracted and 
A

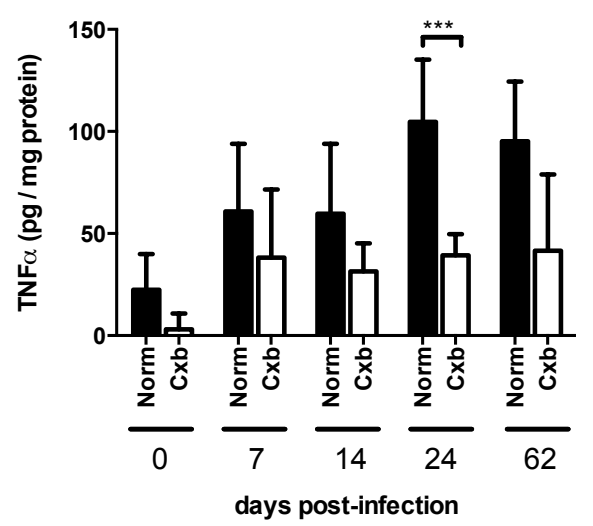

C

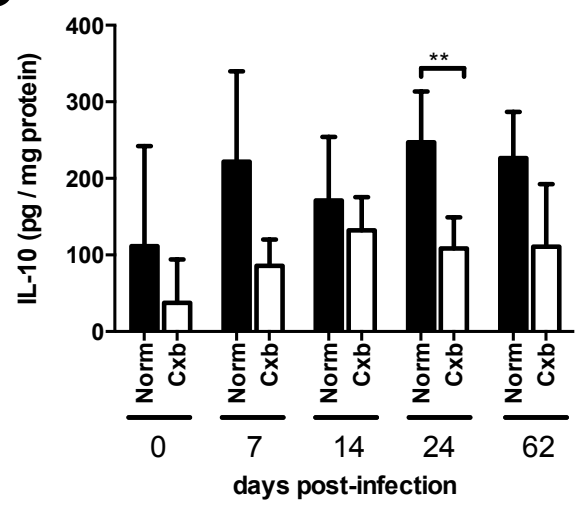

B

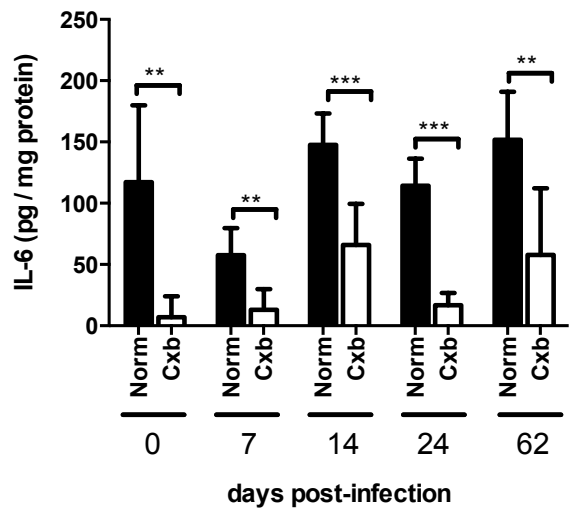

D

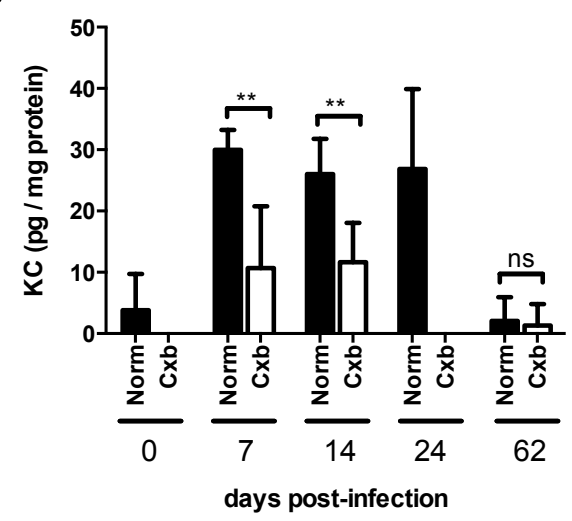

Fig. 4.8. Cytokine and chemokine production in the ankle joints of Celecoxib-fed mice. 30 mice were fed a normal chow diet and 30 mice were fed a diet supplemented with 1000 ppm Celecoxib. Mice were infected with $B b$ and disease was allowed to progress for 62 days. On days $0,7,14,24$ and 62 post-infection, ankle joint tissue was extracted and quantities of TNFa (A), IL-6 (B), IL-10 (C), and KC (D) were detected using ELISA and normalized to total protein concentration quantified using BCA. ${ }^{* *}=P<0.01 .{ }^{* *}=P<0.001$. 
cytokines/chemokines were quantified using ELISA. As previously observed, $\mathrm{COX}-2^{-/-}$mice exhibited reduced ankle joint swelling compared to WT littermates

(fig. 4.9 A). Furthermore, all of the cytokines/chemokines assessed trended lower in the COX-2-/- compared to WT littermates (fig. 4.9 B - E). Although statistical significance was not reached in these assays, it is possible that if more mice were included in the sample size, significance would be achieved.

These studies indicate that along with effects on eicosanoid production, inhibition of COX-2 results in diminished cytokine/chemokine production. As many eicosanoids produced via the COX-2 pathway are known to induce cytokine production [e.g.: PGE 2 induces IL-6 and IL-10 production (203, 206208)], it is possible that the reduced production of cytokines resulted from the reduced production of COX-2-derived eicosanoids.

\section{DISCUSSION}

This study highlights the effects of COX-2 inhibition on the mechanisms involved in the progression and resolution of Lyme arthritis. Previously, It was shown that inhibition of COX-2 results in the inability of mice infected with $B b$ to resolve arthritis (141). This study indicated that COX-2 inhibition affects the function of numerous biosynthetic pathways and the production of cytokines and chemokines. However, agonism of the $\mathrm{PGE}_{2}$ receptor EP2 using Butaprost appeared to effectively restore resolution to WT levels in $\mathrm{COX}_{-} 2^{-/-}$mice, suggesting that disruption of this pathway via COX-2 inhibition is responsible for defective resolution mechanisms. 


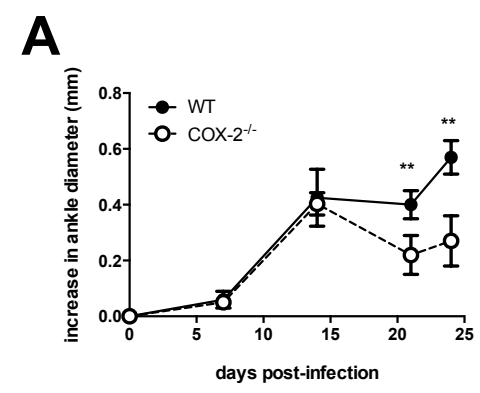

B
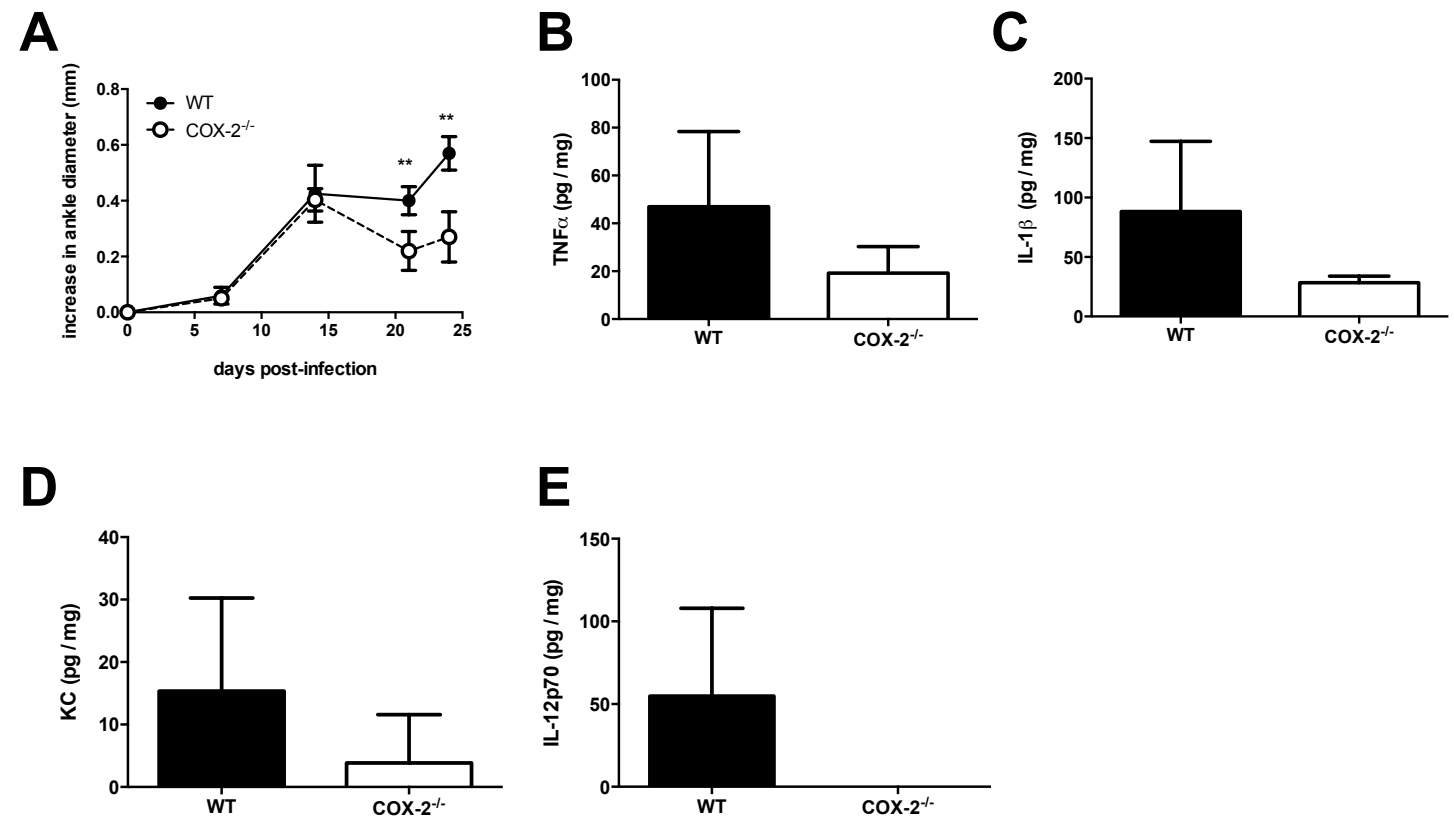

Fig. 4.9. Cytokine and chemokine production in $\mathrm{COX}^{-/-}$mice. 4 WT mice and $4 \mathrm{COX}-2^{--}$mice were infected with $\mathrm{Bb}$. Disease was allowed to progress for 24 days, during which time ankle swelling was monitored using metric calipers (A). On day 24 post-infection, ankle joint tissue was collected and TNFa (B), IL$1 \beta(C), K C(D)$, and IL-12p70 (E) expression was quantified using ELISA and normalized to total joint protein quantified using BCA. ${ }^{* *}=\mathrm{P}<0.01$. 
It is important to note that the study presented here, in which arthritis severity, eicosanoid production, and cytokine and chemokine production were monitored throughout the course of Lyme arthritis in mice fed a diet supplemented with Celecoxib, had methods and results that differed in significant ways from those conducted and reported by Blaho, et al. (141). For example, in the study by Blaho, et al., mice fed the Celecoxib diet failed to resolve arthritis by day 35 post-infection. However, in the study presented here, by day 62 postinfection, Celecoxib-fed mice had fully resolved arthritis. In fact, by day 24 postinfection, severity was significantly reduced in Celecoxib-fed mice when compared to controls. It is possible that if a time point was taken at day 35 postinfection arthritis severity would be similar in Celecoxib-fed and control mice, as described by Blaho, et al. If so, it would suggest that Celecoxib does not prevent the resolution of arthritis, but delays it. Furthermore, in the study by Blaho, et al., feeding of the experimental diet started on day -1 of $B b$ infection. However, in the study presented here, feeding of the experimental diet began 2 weeks prior to infection with $B b$. It is possible that extending the regimen of Celecoxib resulted in activation of compensatory mechanisms that this resulted in different findings.

It is intriguing that although severity and type scores were unaltered in the Celecoxib-fed and $\mathrm{COX}-2^{-/-}$mice during the peak of severity, the expression of several powerful cytokines and chemokines was reduced. Although type scores, which measure immune cell infiltration, were also unaltered, it is possible that the immune response mounted in the absence of COX-2 was phenotypically different compared to the immune response in control animals. Eicosanoids are known to 
play important roles in the recruitment and activation of immune cells, and as COX-2 inhibition dynamically affected the production of eicosanoids, it is likely that the recruitment and activation of the subsequent immune response was affected as well. Although macrophages and neutrophils were recruited during the immune response to $B b$ in the Celecoxib-fed or $C O X-2^{-/-}$mice similar to control and WT mice, alterations in the eicosanoid milieu may have impacted the activation of these cells and subsequent cytokine and chemokine production, explaining the effects seen in figures 4.8 and 4.9 .

Further studies are required to clarify the exact mechanisms that are involved in the immune response during COX-2 inhibition. It would be beneficial to repeat the Celecoxib experiment with more mice. Furthermore, in order to translate results, it would be beneficial to start treatment one day prior to infection with $B b$ similar to the studies conducted by Blaho, et al. Repeating the studies in which $\mathrm{COX}-2^{-/-}$mice are used would also be beneficial, especially if more mice were available and if a time course was possible. Lastly, as the treatment of COX $-2^{-/-}$mice with Butaprost appeared to enable these mice to resolve arthritis, it would be valuable to repeat this experiment to see if these results were repeatable. If so, it could be confirmed that the $\mathrm{PGE}_{2} / \mathrm{EP} 2$ axis is responsible for resolution during Lyme arthritis. 


\section{CHAPTER FIVE}

\section{THE EFFECT OF DIETARY FISH OIL SUBSTITUTION ON EXPERIMENTAL LYME DISEASE}

\section{INTRODUCTION}

Eicosanoids are derived from polyunsaturated fatty acids (PUFA) and are involved in many biological processes (209) including the modulation of both innate and adaptive immunity. Eicosanoids are generated when PUFA are liberated from membrane phospholipids via phospholipase $A_{2}\left(P L A_{2}\right)$ and metabolized via a variety of enzymes, most notably the cyclooxygenases (COX-1 and COX-2) and the lipoxygenases (5-, 12-, and 15-LOX).

A traditional Western diet results in the majority of dietary fatty acids (FA) being derived from $\omega-6$ PUFA, including arachidonic acid (AA) and linoleic acid (LA). Growing evidence suggests that incorporation of $\omega-3$ FA derived from fish oil (FO) into the diet may be beneficial for the alleviation of a variety of immune disorders including cardiovascular disease, diabetes, rheumatoid arthritis, cystic fibrosis, and cancer $(81,210-214)$. However, the mechanism by which FO mediates its beneficial effects remains unclear. As eicosanoids derived from FO are less bioactive than their $\omega$-6-derived counterparts, it is suggested that the substitution of FO-derived FA into membrane phospholipids results in a less antiinflammatory microenvironment (215-219). Recent data also suggests that FOderived FA can directly inhibit inflammation-inducing mechanisms such as NF- 
$\mathrm{KB}$-induced inflammasome activation and inducible nitric oxide synthase (iNOS) expression $(220,221)$.

We have previously used a high-throughput methodology to detect the temporal production of 139 eicosanoids throughout the course of experimental murine Lyme arthritis $(144,145)$. AA-derived eicosanoids are known to play a role in the development and resolution of Lyme arthritis (fig. 5.1) and inflammation resulting from $B b$ infection is commonly treated with COX-2-specific non-steroidal anti-inflammatory drugs (NSAIDs) (222). As FO-derived eicosanoids are believed to dampen inflammation, we wanted to assess the effects of dietary FO substitution on inflammation resulting from infection with $B b$. Therefore, in the present study, we developed a diet in which w-6-containing soy oil (SO, the FA source in typical murine diets) was replaced with $\omega$-3-containing Menhaden fish oil and assessed the effects of this substitution on eicosanoid generation and Lyme arthritis and carditis severity.

\section{RESULTS}

\section{A. Dietary fish oil substitution alters the serum and liver fatty acid profile.}

To determine the effect of dietary fish oil substitution on total fatty acid (FA) composition, we constructed a rodent chow diet in which Menhaden fish oil was the primary source of fatty acids (FO diet). Control animals were fed a normal chow diet in which soy oil was the primary source of fatty acids (SO diet). After two weeks, the serum and liver fatty acid composition of mice fed either the FO or SO diets was determined (Table 5.1). The $\omega-6$ fatty acids arachidonic 


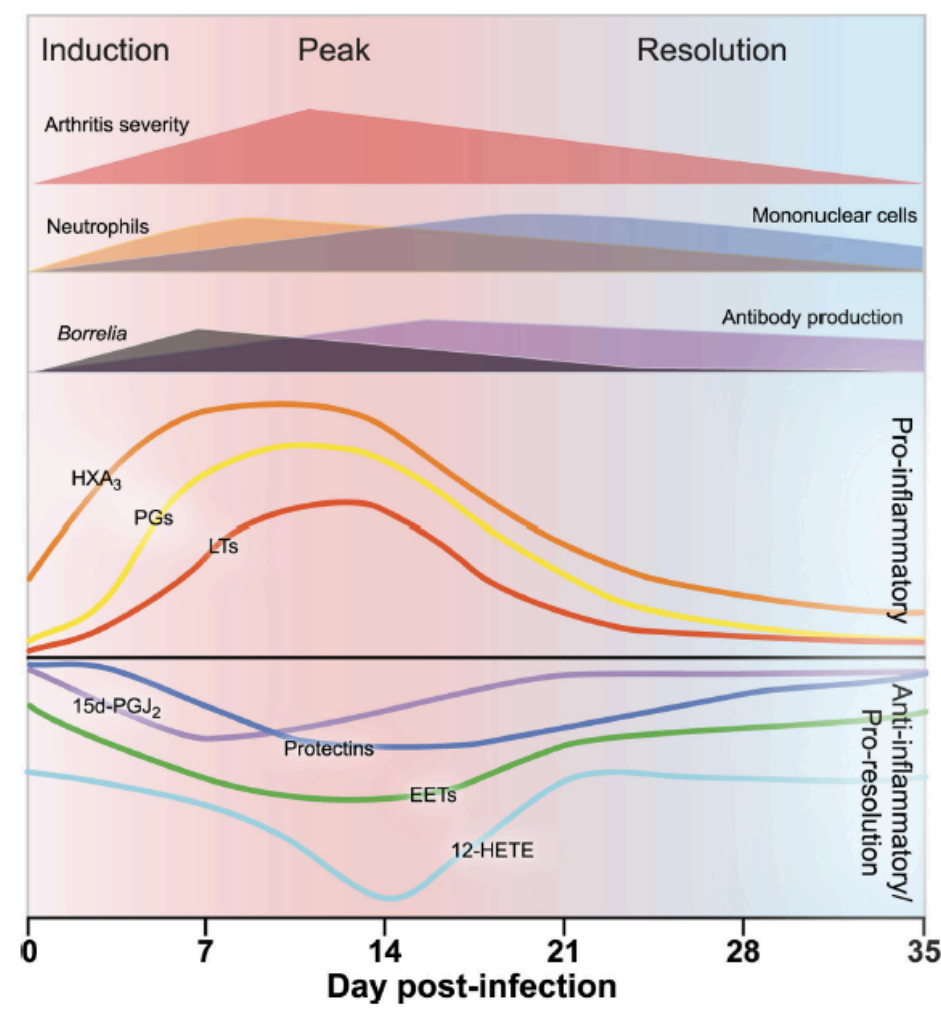

Fig. 5.1. Eicosanoids regulate all phases of Lyme arthritis (144). Proinflammatory cyclooxygenase (COX)-2-derived prostaglandins (PGs) such as $P G E_{2}$ and $P G D_{2}$ initiate the immune response to $B b$ while $15 d-P G J_{2}$ limits the severity of subsequent inflammation. Lipoxygenase (LOX)-derived hepoxylin $B_{3}$ $\left(\mathrm{HXB}_{3}\right)$ and leukotrienes (LTs) mediate the recruitment of neutrophils and mononuclear cells to sites of infection. The production of DHA-derived protectins mediates the resolution of inflammation. This information is based on a lipidomic analysis of the temporal production of eicosanoids throughout the course of Lyme arthritis detected using LC-MS/MS conducted by Blaho, et al. 2009 (144). (Figure generated by Dr. Victoria A. Blaho.) 


\begin{tabular}{|c|c|c|c|c|}
\hline \multirow[b]{2}{*}{ Fatty acid } & \multicolumn{2}{|c|}{ Serum } & \multicolumn{2}{|c|}{ Liver } \\
\hline & SO & FO & so & FO \\
\hline & \multicolumn{4}{|c|}{ mol\% of total fatty acids } \\
\hline 16:0 & $23.8 \pm 0.4$ & $30.1 \pm 0.6^{*}$ & $32.7 \pm 0.7$ & $41.8 \pm 0.7^{*}$ \\
\hline 18:0 & $12.8 \pm 0.3$ & $11.2 \pm 0.3^{*}$ & $23.0 \pm 0.7$ & $19.9 \pm 0.7^{*}$ \\
\hline $18: 1(n-9)$ & $8.4 \pm 0.6$ & $8.8 \pm 0.4$ & $7.7 \pm 0.5$ & $6.3 \pm 0.5^{*}$ \\
\hline $18: 2(n-6)$ & $32.8 \pm 0.4$ & $20.0 \pm 1.1^{*}$ & $16.2 \pm 0.2$ & $10.0 \pm 0.5^{*}$ \\
\hline $18: 3(n-3)$ & $0.5 \pm 0.1$ & $0.1 \pm 0.1$ & $0.1 \pm 0.0$ & $0.0 \pm 0.0$ \\
\hline $20: 3(n-6)$ & $0.6 \pm 0.1$ & $0.3 \pm 0.1$ & $0.7 \pm 0.1$ & $0.6 \pm 0.0$ \\
\hline $20: 4(n-6)$ & $17.0 \pm 0.5$ & $9.6 \pm 0.5^{*}$ & $14.0 \pm 0.5$ & $6.1 \pm 0.3^{*}$ \\
\hline $20: 5(n-3)(E P A)$ & $0.0 \pm 0.0$ & $7.7 \pm 0.6^{*}$ & $0.2 \pm 0.0$ & $3.6 \pm 0.4^{*}$ \\
\hline $22: 5(n-3)$ & $0.0 \pm 0.0$ & $0.3 \pm 0.1$ & $0.1 \pm 0.0$ & $0.4 \pm 0.0$ \\
\hline 22:6(n-3) (DHA) & $3.3 \pm 0.1$ & $8.7 \pm 0.5^{*}$ & $5.0 \pm 0.2$ & $8.4 \pm 0.4^{*}$ \\
\hline
\end{tabular}

Table 5.1. Dietary fish oil substitution alters the serum and liver fatty acid profile (145). Mice were fed either a control diet in which fatty acids (FA) were derived from soy oil (SO) or a diet in which Menhaden fish oil was the primary fatty acid source (FO). After two weeks on the diets, serum and liver fatty acids were determined by Dr. Kevin L. Fritsche. Values represent mean $\pm \mathrm{SEM}, n=10$ mice. * denotes that concentration of FA in the FO-fed mice differs significantly from concentration of FA in the SO-fed mice within individual tissues, $p<0.05$. Fatty acids that constituted less than $0.5 \%$ of total fatty acids were not included on this table. (Values obtained and calculated and table constructed by Dr. Kevin L. Fritsche.) 
acid (AA) and linoleic acid (LA) were the major fatty acids found in the serum and liver of SO diet-fed mice, with no traces of the fish oil-derived $\omega-3$ fatty acids eicosapentaenoic acid (EPA) or docosapentaenoic acid (DHA). When mice were fed the FO diet, serum and liver fatty acids were mainly comprised of EPA and DHA and levels of AA and LA were decreased. This data indicated that feeding mice a diet in which FO is the primary FA source for two weeks led to alterations in the total fatty acid profile, as production of $\omega-6$ fatty acids was significantly reduced and $\omega-3$ fatty acids comprised the majority of total fatty acids.

\section{B. Dietary fish oil substitution alters global eicosanoid production.}

To determine the effect of fish oil substitution on arthritic inflammation, we fed mice the SO or FO diets for two weeks prior to infecting them with $B b$. On days 0,10 , and 21 post-infection, we isolated lipids from joint tissue and characterized the production of 103 distinct eicosanoid species using LC-MS/MS (Table 5.2) (144). At day 0 , eicosanoid production did not differ between SOand FO-fed mice. However, as the infection progressed, metabolite production varied significantly. The production of 30 metabolites was different between SOand FO-fed mice at day 10 post-infection and production of 76 metabolites varied between the two diets day 21 post-infection.

To better visualize the changes in eicosanoid species produced throughout infection in either diet, we constructed a heat map displaying fold

change in eicosanoid concentrations relative to day 0 (fig. 5.2 A). In SO-fed mice, AA- and LA-derived metabolites increased up to 4-fold throughout 


\begin{tabular}{|c|c|c|c|c|c|c|}
\hline & \multicolumn{3}{|c|}{ so } & \multicolumn{3}{|c|}{ FO } \\
\hline & $0 \mathrm{~d}$ & $10 \mathrm{~d}$ & $21 \mathrm{~d}$ & $0 \mathrm{~d}$ & $10 \mathrm{~d}$ & $21 \mathrm{~d}$ \\
\hline$n$ & 4 & 4 & 9 & 4 & 4 & 9 \\
\hline \multicolumn{7}{|l|}{ Eicosanoid subclass } \\
\hline & \multicolumn{6}{|c|}{$n g / m g$ of tissue } \\
\hline PG & $0.03 \pm 0.01$ & $0.04 \pm 0.01$ & ${ }^{*} 0.14 \pm 0.02$ & $0.04 \pm 0.01$ & ${ }^{*} 0.11 \pm 0.03$ & ${ }^{*} 0.13 \pm 0.02$ \\
\hline Mono-hydroxylated & $0.32 \pm 0.15$ & $0.92 \pm 0.15$ & $2.03 \pm 0.22$ & $0.47 \pm 0.22$ & ${ }^{*} 1.42 \pm 0.44$ & $* 1.44 \pm 0.22$ \\
\hline Di-hydroxylated & $0.00 \pm 0.00$ & $0.01 \pm 0.00$ & ${ }^{*} 0.02 \pm 0.00$ & $0.01 \pm 0.00$ & $0.01 \pm 0.00$ & ${ }^{*} 0.01 \pm 0.00$ \\
\hline Hepoxilins & $0.00 \pm 0.00$ & ${ }^{*} 0.05 \pm 0.01$ & ${ }^{*} 0.11 \pm 0.01$ & $0.00 \pm 0.00$ & ${ }^{*} 0.04 \pm 0.01$ & ${ }^{*} 0.05 \pm 0.01$ \\
\hline Lipoxins, resolvins, and protectins & $0.00 \pm 0.00$ & ${ }^{*} 0.00 \pm 0.00$ & $0.01 \pm 0.00$ & $0.00 \pm 0.00$ & ${ }^{* *} 0.01 \pm 0.00$ & ${ }^{*} 0.01 \pm 0.00$ \\
\hline Epoxide & $0.06 \pm 0.03$ & $0.05 \pm 0.01$ & ${ }^{*} 0.15 \pm 0.02$ & $0.09 \pm 0.00$ & ${ }^{*} 0.21 \pm 0.06$ & $0.18 \pm 0.02$ \\
\hline Nonenzymatic & $0.05 \pm 0.02$ & $0.03 \pm 0.01$ & $0.07 \pm 0.01$ & $0.05 \pm 0.02$ & ${ }^{*} 0.27 \pm 0.06$ & ${ }^{*} 0.29 \pm 0.04$ \\
\hline Totals & $0.05 \pm 0.02$ & $1.10 \pm 0.18$ & ${ }^{*} 2.35 \pm 0.28$ & $0.66 \pm 0.29$ & ${ }^{*} 2.08 \pm 0.60$ & ${ }^{*} 2.09 \pm 0.32$ \\
\hline
\end{tabular}

\section{Table 5.2. Dietary fish oil substitution results in a global shift in eicosanoid}

production (145). Table displays total eicosanoid concentrations in the joints of soy oil (SO)- or fish oil (FO)-fed mice at 0,10 , and 21 days post-infection. $n=4$ at days 0 and 10 and 9 at day 21 post-infection. Values are total eicosanoid concentration ( $\mathrm{ng} / \mathrm{mg}$ tissue) \pm SEM. \# denotes that a value is significantly different from day 0 within a diet while ${ }^{*}$ denotes a significant difference in eicosanoid concentration between the SO and FO diet at a certain time point. $p$ $<0.05$. (Eicosanoid concentrations determined and table constructed by Dr. Darren S. Dumlao.) 


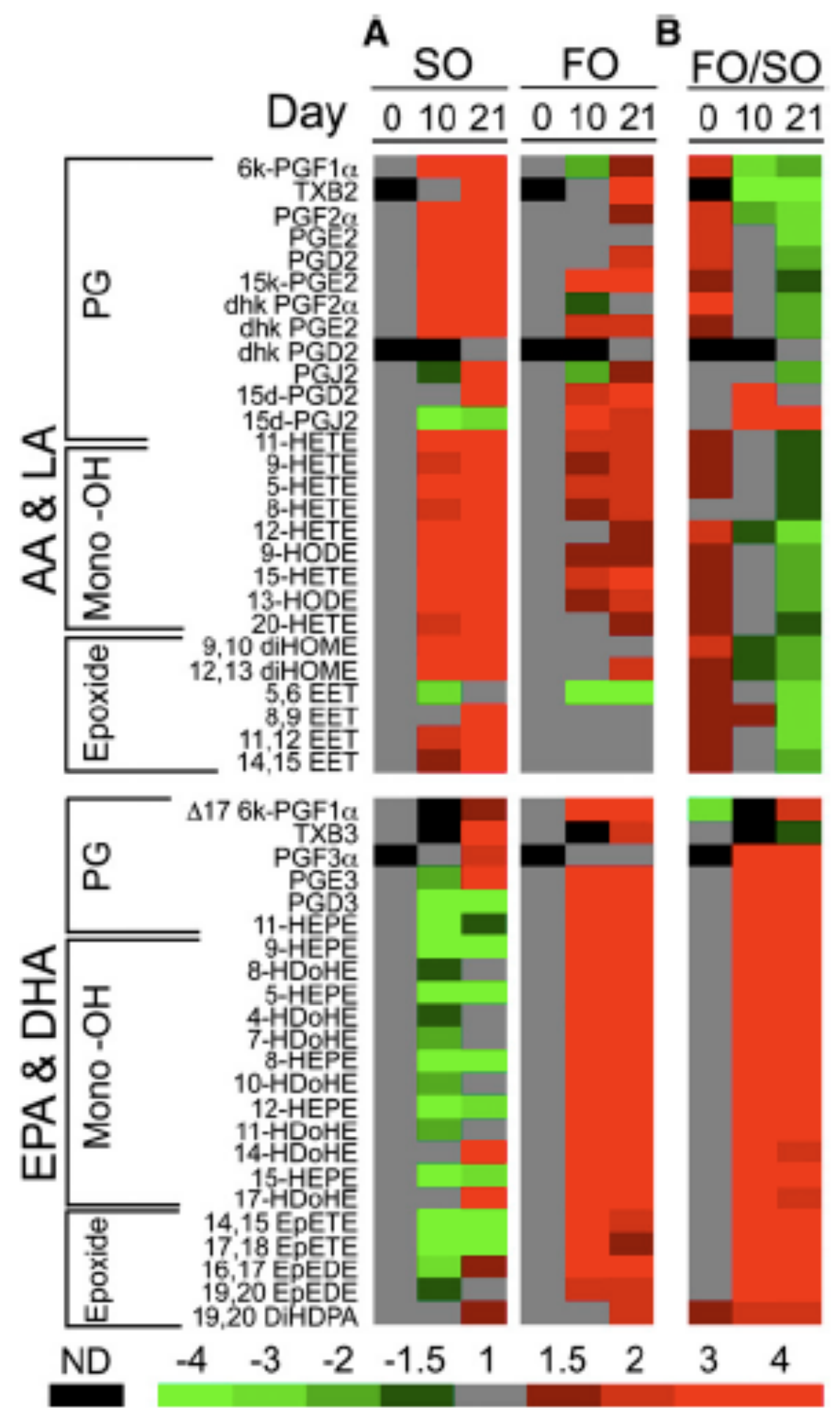


Fig. 5.2. Dietary fish oil substitution results in a global shift in eicosanoid generation (145). Heat map displays the relative fold change of select AA-, LA-, EPA-, and DHA-derived eicosanoids in the joints of SO- or FO-fed mice at days 0, 10, and 21 post-infection. Eicosanoids are grouped based on subclass. (A) Fold change in eicosanoids compared to day 0 in SO- or FO-fed mice. For eicosanoids that were ND on day 0 , the relative fold change was determined from the day that they first were detected. (B) Comparison of the relative eicosanoid concentrations detected in FO-fed mice versus SO-fed mice. ND (black) indicates that an eicosanoid was either not detected or was detected in concentrations below the limit of detection. $n=4$ at days 0 and 10 post-infection and 9 at day 21 post-infection. (Heat map composed by Dr. Darren S. Dumlao.) 
the course of infection, while EPA- and DHA-derived metabolites remained relatively unchanged. In FO-fed mice, increases in AA- and LA-derived metabolites were not nearly as significant (1.5 - 2-fold on average), while EPAand DHA-derived metabolites increased up to 4-fold throughout the course of infection. That data suggested that FO substitution led to a drastic increase in the production of EPA- and DHA-derived metabolites and decreased production of AA- and LA-derived metabolites.

To more directly visualize the effect of the FO diet on eicosanoid production, we constructed a heat map showing relative fold change in eicosanoid production in mice fed the FO diet relative to those fed the SO diet (fig. 5.2 B). We found that FO substitution led to a global shift in eicosanoid production, from AA- and LA-derived metabolites to EPA- and DHA-derived metabolites. Expression of most EPA- and DHA-derived metabolites were increased approximately 4-fold over levels found in the SO-fed mice and the majority of AA- and LA-derived metabolites were significantly decreased when mice were fed the FO diet compared to those fed the SO diet. At day 10 postinfection, EPA- and DHA-derived metabolites accounted for approximately $47 \%$ of eicosanoids produced in the FO-fed mice, compared to $11 \%$ in the SO-fed mice. At day 21 post-infection, the effect of FO substitution was even more apparent, as EPA- and DHA-derived metabolites accounted for approximately $59 \%$ of total eicosanoids produced, compared to only $9 \%$ in the SO-fed mice. This data indicated that FO substitution led to a global shift in eicosanoid 
production and that the majority of eicosanoid species produced in FO diet-fed mice were derived from EPA and DHA.

\section{Mono-hydroxylated fatty acid metabolites are produced primarily non- enzymatically irrespective of diet.}

AA-derived mono-hydroxylated metabolites (MHM) (HETEs) are vasoconstrictive and induce the development of edema (223). EPA- and DHAderived MHM (HEPEs and HDoHEs) are precursors to the anti-inflammatory Eseries and D-series resolvins, respectively $(224,225)$. Thus, AA-derived MHM are considered pro-inflammatory while EPA- and DHA-derived MHM are considered anti-inflammatory, suggesting another mechanism by which FOderived FA may induce an anti-inflammatory microenvironment.

We found that AA-derived MHM were increased, albeit to low levels, in SO-fed mice throughout the course of $B b$ infection (shown: 5-HETE, fig. 5.3), but were decreased in comparison in the FO-fed mice by day 21 post-infection. EPA-derived MHM (shown: 5-HEPE) and DHA-derived MHM (shown: 4-HDoHE) were found at low levels throughout the course of $B b$ infection in SO-fed mice, but were dramatically increased in comparison in the FO-fed mice at days 10 and 21 post-infection. These data suggested that EPA and DHA were the preferred substrates for 5-LOX for the production of MHM in the FO-fed mice.

MHM are unique in that they can be produced by two distinct mechanisms: enzymatically by lipoxygenases (LOX) or cytochrome p450 mono- 


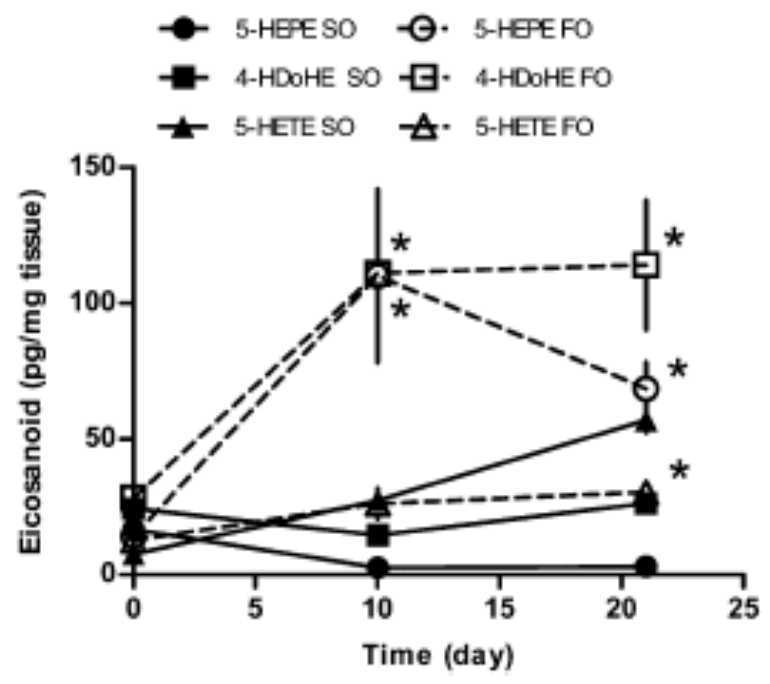

Fig. 5.3. Dietary fish oil substitution results in increased levels of EPA- and DHA-derived mono-hydroxylated metabolites (MHM) (145). Quantitative amounts of representative MHM produced in SO- or FO-fed mice throughout the course of $B b$ infection. Values of AA-derived 5-HETE, EPA-derived 5-HEPE, and DHA-derived 4-HDoHe are means \pm SEM. $n=4$ on days 0 and 10 post-infection and 9 on day 21 post-infection. \# denotes a significant difference from day 0 within each diet and ${ }^{*}$ denotes a significant difference in the concentrations of the eicosanoid in the FO-fed mice compared to the SO-fed mice. $p<0.05$. (Quantification of eicosanoids and construction of figure by Dr. Darren S. Dumlao.) 
oxygenase-1 (CYP1) $(224,226)$ or non-enzymatically by reactive oxygen species (ROS) (227-229). In order to determine the contribution of non-enzymatic mechanisms to the generation of MHM, we performed chiral LC-MS analysis. Enzymatic metabolism of fatty acids results in the production of metabolites in the S-isomeric state, while non-enzymatic metabolism yields a racemic (1:1) mixture of R- and S-isomers. EPA- and DHA-derived R and $\mathrm{S}$ isomers were produced at ratio of $1: 1$, indicating that these metabolites were being produced primarily via non-enzymatic mechanisms (fig. 5.4). The most abundant MHM produced, LA-derived 9- and 13-hydroxyoxtadecadienioc acid (9- and 13-HODE), were also found in a racemic mixtures, indicating that the majority of AA-, LA-, EPA-, and DHA-derived MHM were being produced as a result of non-enzymatic metabolism, perhaps as a result of ROS generated in response to $B b$ infection.

When we assessed the production of metabolites produced solely by nonenzymatic mechanisms, we found that the total amounts of non-enzymatically produced metabolites were increased in the FO-fed mice at day 10 and 21 postinfection (Table 5.3). Further, the majority of non-enzymatically produced metabolites were derived from either EPA or DHA, suggesting that these fatty acids are more susceptible to oxidative stress than AA or LA. Notably, isoprostanes, which are notorious for generation via non-enzymatic mechanisms $(230,231)$, were found at low levels regardless of diet, suggesting that they were produced primarily via cyclooxygenase (COX). 


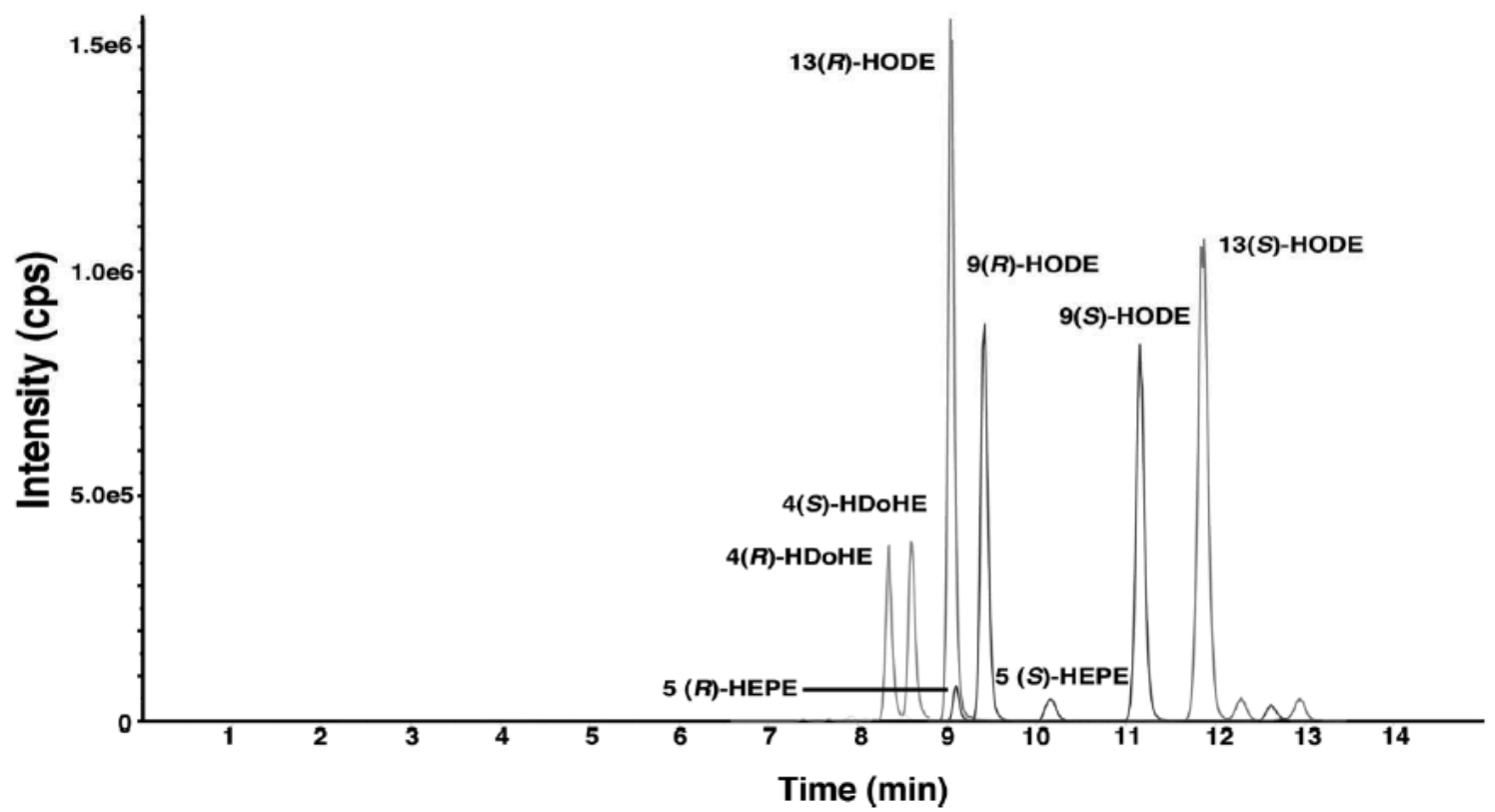

Fig. 5.4. The majority of mono-hydroxylated metabolites produced in fish oil-fed mice are generated non-enzymatically (145). Representative chiral LC-MS/MS-generated chromatograph of MHM produced in the joint of a FO-fed mouse at day 21 post-infection. Proper elution times were determined using commercially available standards for the R- and S-isomers of the individual eicosanoids. Data is representative of 5 replicate samples. (LC-MS/MS conducted and chromatograph generated by Dr. Darren S. Dumlao.) 


\begin{tabular}{|c|c|c|c|c|c|c|}
\hline Diet & \multicolumn{3}{|c|}{ Soy Oil } & \multicolumn{3}{c|}{ Fish Oil } \\
\hline $\begin{array}{c}\text { Day } \\
\boldsymbol{n}\end{array}$ & $\mathbf{0}$ & $\mathbf{1 0}$ & $\mathbf{2 1}$ & $\mathbf{0}$ & $\mathbf{1 0}$ & $\mathbf{2 1}$ \\
\hline Eicosanoid & & $\mathbf{4}$ & $\mathbf{9}$ & $\mathbf{4}$ & $\mathbf{4}$ & $\mathbf{9}$ \\
\hline 5-iso-PGF $2 \alpha \mathrm{VI}$ & $0.2 \pm 0.1$ & $0.7 \pm 0.1$ & $1.2 \pm 0.1$ & $0.4 \pm 0.1$ & $0.8 \pm 0.3$ & $0.7 \pm 0.1$ \\
\hline 8-iso-PGF $2 \alpha \mathrm{III}$ & $0.2 \pm 0.1$ & $0.4 \pm 0.1$ & $1.0 \pm 0.1$ & $0.3 \pm 0.0$ & $0.4 \pm 0.2$ & $0.5 \pm 0.1$ \\
\hline 9-HETE & $0.2 \pm 0.1$ & $8.2 \pm 1.5$ & $17.7 \pm 1.4$ & $5.0 \pm 1.6$ & $9.6 \pm 2.4$ & $11.5 \pm 1.8$ \\
\hline 9-HEPE & $9.9 \pm 5.0$ & $0.8 \pm 0.1$ & $1.3 \pm 0.2$ & $8.6 \pm 24.5$ & $50.6 \pm 14.0$ & $36.2 \pm 6.1$ \\
\hline 8-HDoHe & $11.1 \pm 4.9$ & $7.0 \pm 1.0$ & $11.5 \pm 0.8$ & $10.6 \pm 3.8$ & $62.0 \pm 13.1$ & $67.5 \pm 9.1$ \\
\hline 16-HDoHe & $5.8 \pm 2.4$ & $4.6 \pm 0.6$ & $8.6 \pm 0.6$ & $6.4 \pm 2.1$ & $40.6 \pm 8.7$ & $48.6 \pm 7.0$ \\
\hline 20-HDoHe & $12.8 \pm 5.3$ & $9.9 \pm 1.3$ & $18.4 \pm 1.3$ & $14.1 \pm 4.8$ & $77.8 \pm 16.2$ & $92.3 \pm 12.8$ \\
\hline
\end{tabular}

Table 5.3. EPA- and DHA-derived MHM are increased in fish oil-fed mice (145). LC-MS/MS-determined eicosanoid levels in joint of mice fed the fish oil or soy oil diets on days 0,10 , and 21 post-infection. Values represent mean \pm SEM. (Values determined by Dr. Darren S. Dumlao. Table created by Anna M Cunningham based on a table generated by Dr. Darren S. Dumlao.) 
Taken together, this data suggested that $B b$ infection induced a substantial amount of oxidative stress that resulted in non-enzymatic metabolism of MHM. Furthermore, EPA- and DHA-derived MHM were more susceptible to non-enzymatic metabolism in this model. As metabolism of EPA- and DHAderived MHM results in the production of putative anti-inflammatory metabolites, this data suggests a mechanism whereby dietary fish oil may induce an antiinflammatory microenvironment.

\section{Dietary fish oil substitution alters COX metabolite production.}

Cyclooxygenase (COX)-2 metabolites are known to play a large role in the immune response to $B b(141,143)$ and inflammation in general (232). Furthermore, in RAW264.7 macrophages, it has been shown that EPA and DHA treatment results in inhibition of both COX-1 and COX-2 $(233,234)$ and a preferential usage of substrate (AA) by the lipoxygenase (LOX) pathway, leading to increased production of leukotrienes and other lipoxygenase metabolites (235). Therefore, we wanted to assess the effect of dietary FO substitution on this pathway during experimental Lyme disease.

6-keto-prostaglandin $\mathrm{F}_{2 \alpha}\left(6 \mathrm{k}_{-}-\mathrm{PGF}_{2 \alpha}\right)$ is a stable breakdown production of the potent, but very bioactive vasodilator, $\mathrm{PGI}_{2}$. We found that levels of $6 \mathrm{k}$ $\mathrm{PGF}_{2 a}$ were increased throughout $B b$ infection in the joints of SO-fed mice, but were reduced by $71 \%$ in the joints of mice fed the FO-diet by day 21 postinfection (fig. 5.5). $\quad \mathrm{TXB}_{2}$, a stable breakdown product of the vasoconstrictive $\mathrm{TXA}_{2}$, was 
found at low levels throughout the course of disease in joints of SO-fed mice, but was found at even lower levels the joints of FO-fed mice. Surprisingly, the EPAderived analogs of these molecules, $\Delta 17,6 \mathrm{k}-\mathrm{PGF}_{1 \alpha}$ and $\mathrm{TXB}_{3}$, were not detected in the FO-fed mice. These data suggested that FO substitution reduced the production of AA-derived vaso-regulatory eicosanoids by COX-2, but perhaps by mechanisms other than providing alternative substrates such as direct inhibition $(233,234)$.

$\mathrm{PGE}_{2}(202-204,208,236-240)$ and $\mathrm{PGD}_{2}$ (241) are two classically immuno-regulatory, AA-derived, COX-2 metabolites. As expected (144), in the SO-fed mice, these metabolites were increased throughout the course of infection (fig. 5.6 A, B). Although these metabolites were also increased throughout the course of infection in FO-fed mice, their production was significantly attenuated at day 21 post-infection compared to the SO-fed mice. The analogous EPA-derived 3-series prostaglandins $\left(\mathrm{PGE}_{3}\right.$ and $\left.P G D_{3}\right)$ were found at low levels throughout the course of infection in SO-fed mice, but were increased significantly in the FO-fed mice (fig. 5.6 C, D). These data suggested that dietary FO substitution resulted in the preferential use of $\omega-3$ substrates by COX-2. As the 3-series PGs are believed to be less bioactive than their AAderived counterparts (216), this could suggest another mechanism by which dietary FO promoted an anti-inflammatory microenvironment. 


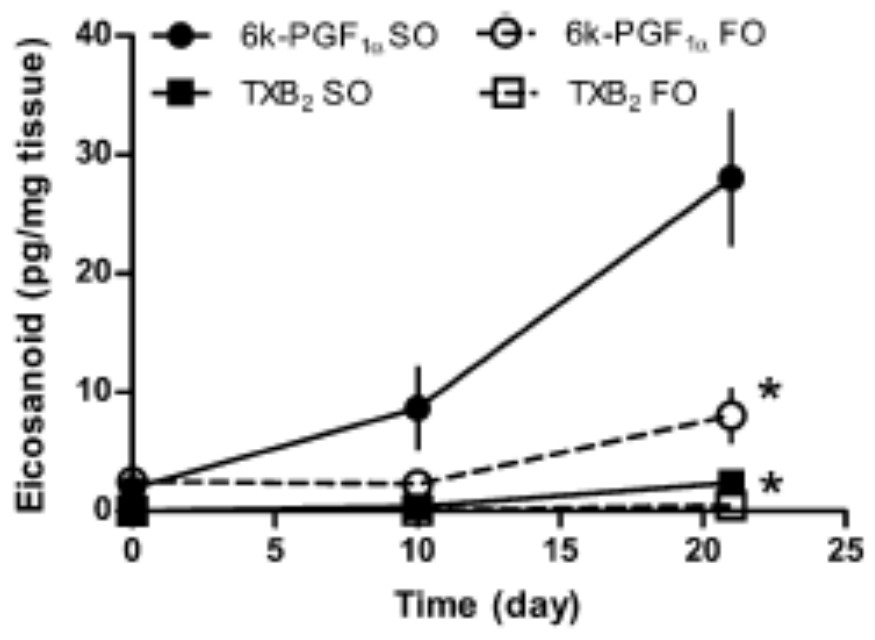

Fig. 5.5. Cyclooxygenase-derived AA metabolites are reduced in fish oil diet-fed mice (145). Amounts of cyclooxygenase (COX)-derived (A) 6-ketoprostaglandin $\mathrm{F}_{1 \alpha}\left(6 \mathrm{k}-\mathrm{PGF}_{1 \alpha}\right)$ and $(\mathrm{B})$ thromboxane $\mathrm{B}_{2}\left(\mathrm{TXB}_{2}\right)$ in the ankles of soy oil (SO)- or fish oil (FO)-fed mice on days 0,10 , and 21 post-infection. Values represent mean \pm SEM, $n=4$ on days 0 and 10 and 9 on day 21 post-infection. * indicates a significant difference in eicosanoids produced in the FO-fed mice compared to the SO-fed mice, $p<0.05$. (Quantification of eicosanoids and generation of figure by Dr. Darren S. Dumlao.) 

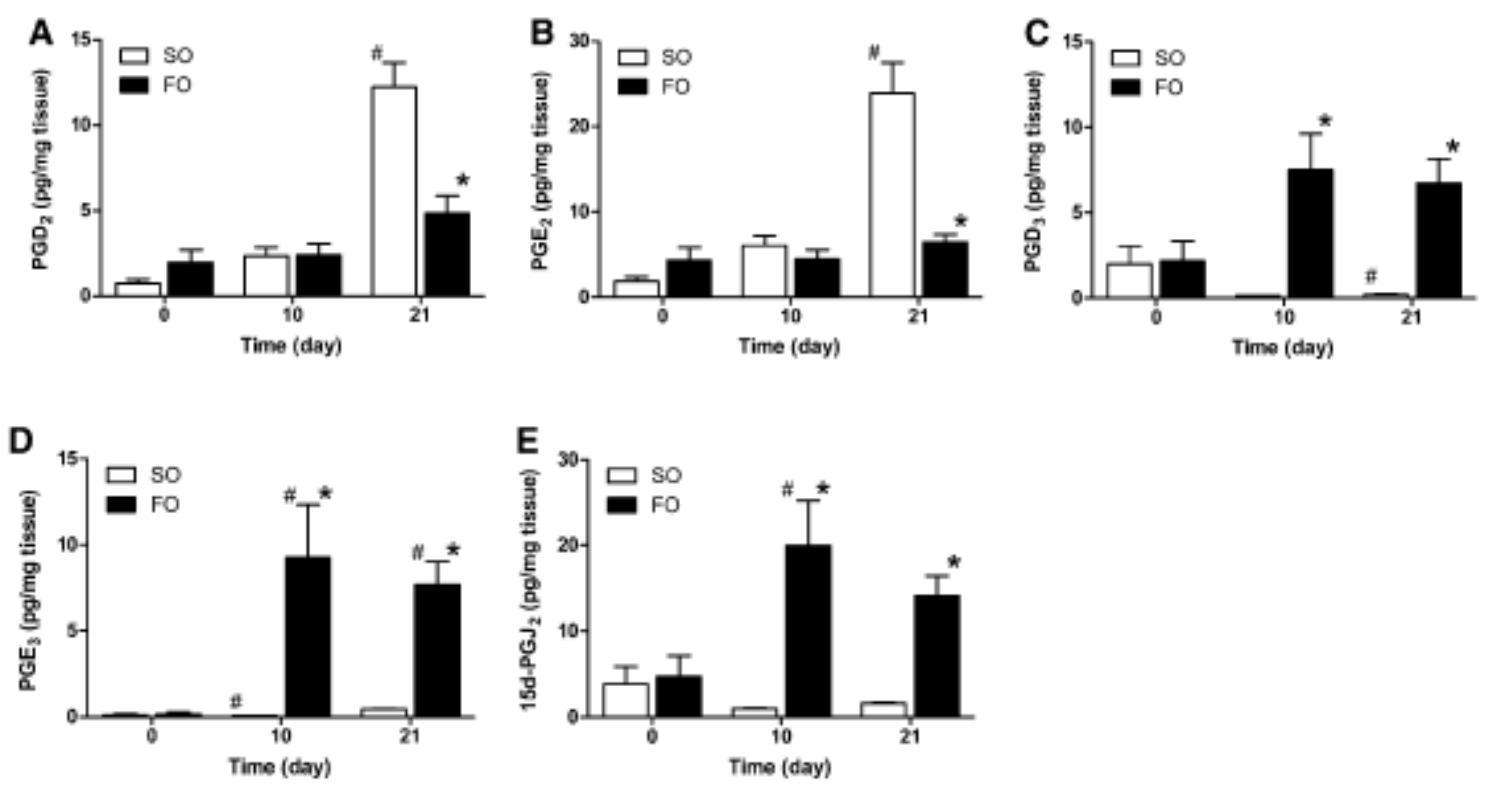

Fig. 5.6. Dietary fish oil substitution results in the preferential use of EPA and DHA substrates for metabolism via cyclooxygenase-2 (145). Cyclooxygenase (COX)-2-derived $\mathrm{PGE}_{2}, \mathrm{PGD}_{2}$, and related metabolites were quantified in the joints of Bb-infected mice fed either SO or FO diets using LCMS/MS on days 0,10 , and 21 post-infection. Values are means \pm SEM, $n=4$ at day 0 and 10 post-infection and 9 on day 21 post-infection. \# denotes a significant difference in metabolite production from day 0 within a diet and * denotes a significant difference in eicosanoid production in the FO-fed mice compared to SO-fed mice, $p<0.05$. (Values determined and figure constructed by Dr. Darren S. Dumlao.) 
Surprisingly, FO-fed mice accumulated high levels of the AA-derived $P G D_{2}$ dehydration product 15-deoxy-PGJ $J_{2}$ (fig. 5.6 E). In fact, levels of $15 \mathrm{~d}$ $P G J_{2}$ in the joints of FO-fed mice accumulated to levels comparable to levels of $\mathrm{PGE}_{2}$ in the joints of SO-fed mice (fig. 5.6 B), suggesting that the FO diet was shunting $A A$ metabolism from $P G E_{2}$ synthesis to $P G D_{2}$ synthesis. To examine this more closely, we calculated the total amount of metabolites detected from the $P G E_{2}$ and $P G D_{2}$ pathways ("PGE 2 total" and "PGD 2 total") in either diet (fig. 5.7). We found that, in the SO-fed mice, $\mathrm{PGE}_{2}$-related products were more abundant that $\mathrm{PGD}_{2}$-related products at days 10 and 21 post-infection (fig. 5.7 A). In the FO-fed mice, the levels of $\mathrm{PGE}_{2}$-related products were decreased in comparison to levels seen in the SO-fed mice, and levels of $\mathrm{PGD}_{2}$-related products were increased (fig. 5.7 B). When we looked at the ratio of $\mathrm{PGE}_{2}$-related products to $\mathrm{PGD}_{2}$-related products in the SO or FO diet, we found that the ratio of $\mathrm{PGE}_{2}$ related products to $P G D_{2}$-related products was significantly decreased at days 10 and 21 post-infection in the FO-fed mice compared to the SO-fed mice (fig. 5.7 C).

These data suggested that dietary FO had drastic effects on the production of mediators through the COX-2 pathway via competitive substrate usage, enzyme inhibition, and preferential PG synthesis. $15 d-P G J_{2}$ is known to have potent anti-inflammatory activities, mainly via signaling through PPARy and inhibition of NF-KB activation $(65-68,241-243)$. The fact that dietary FO substitution resulted in the production of large quantities of this metabolite suggests yet another mechanism by which $\omega-3$ fatty acids are able to induce 

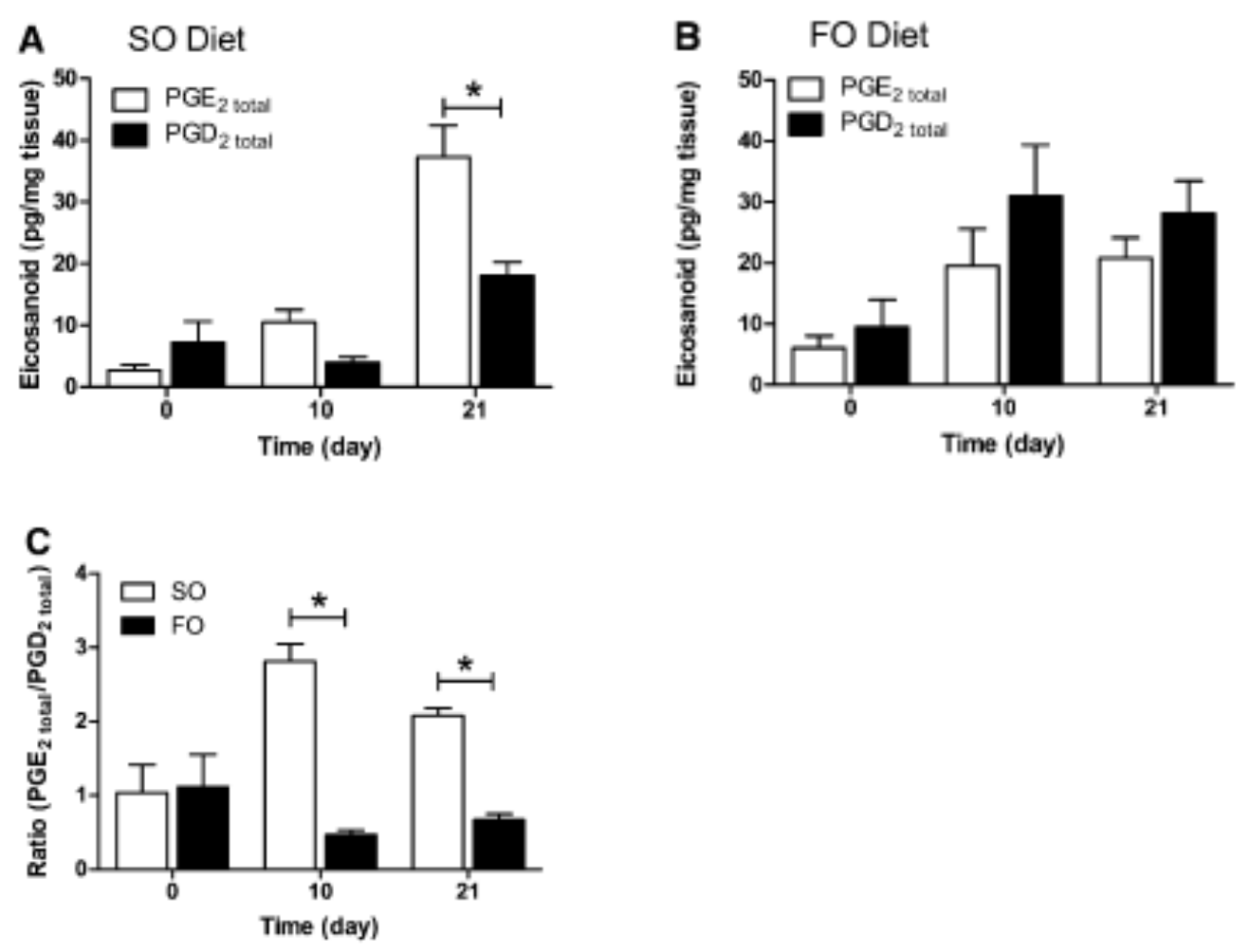

Fig. 5.7. Dietary fish oil substitution results in a redirection of COX-2mediated AA metabolism from $\mathrm{PGE}_{2}$ to $\mathrm{PGD}_{2}$ (145). Total amounts of $\mathrm{PGE}_{2^{-}}$ related products $\left(\mathrm{PGE}_{2}\right.$ total) and $P \mathrm{PD}_{2}$-related products $\left(\mathrm{PGD}_{2}\right.$ total $)$ were determined in the joints of mice fed the (A) soy oil (SO) diet or (B) the fish oil (FO) diet at days 0,10 , and 21 post-infection. Using these values, the ratio of $\mathrm{PGE}_{2}$ total to $\mathrm{PGD}_{2}$ total was determined in the $\mathrm{SO}$ and $\mathrm{FO}$ diets (C). Values are mean SEM, $n=4$ on days 0 and 10 post-infection and 9 on day 21 post-infection. * denotes a significant difference between $\mathrm{PGE}_{2}$ total and $\mathrm{PGD}_{2}$ total $(\mathbf{A}, \mathbf{B})$ and between diets $(\mathbf{C}), p<0.05$. (Values were calculated and figure was developed by Dr. Darren S. Dumlao.) 
an anti-inflammatory microenvironment.

\section{E. Dietary fish oil substitution does not affect Lyme arthritis severity, joint immune cell composition, or joint $B b$ loads.}

Infection $B b$ results in the development of severe inflammatory arthritis that is mediated by neutrophils (134). Eicosanoids are known to play a significant role throughout the course of Lyme arthritis (fig. 5.1) (144). COX-1 metabolites are important for germinal center development and specific antibody production in response to $B b$ infection (143) and COX-2 and 5-LOX are known to play important roles in the resolution of inflammation in this model $(141,142)$. Due to the drastic effects on the eicosanoid profile and the tendency toward the production of molecules that were either less bioactive or anti-inflammatory in nature in response to dietary fish oil substitution, we hypothesized that inflammation resulting from infection with $B b$ would be attenuated. To address this, we fed mice either the FO diet or the SO diet for 2 weeks prior to infection with $1 \times 10^{5} \mathrm{Bb}$ and monitored the progression of ankle swelling (fig. 5.8 A). We found that mice fed the FO diet developed ankle swelling identical to the mice fed the SO diet, indicating that dietary FO substitution does not affect joint swelling in this model.

Although joint swelling is typically indicative of underlying inflammation resulting from $B b$ infection, some exceptions have been noted $(142,153)$. Thus, 
A

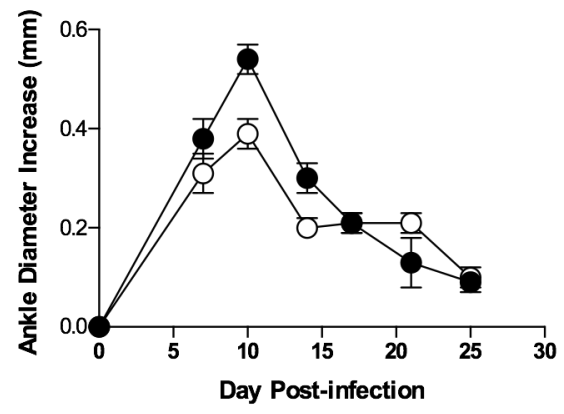

C

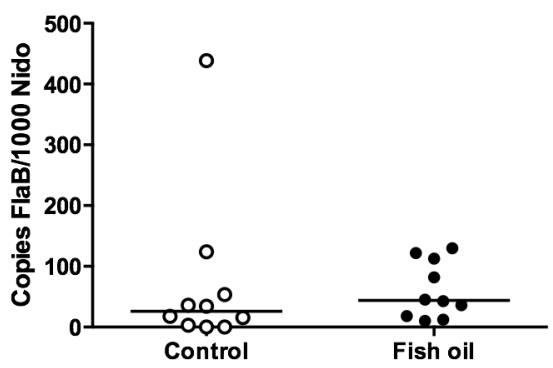

B

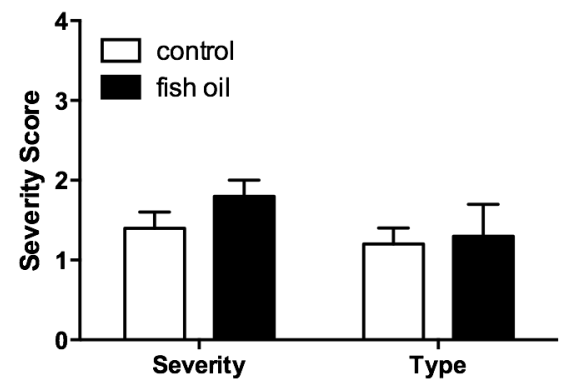

Fig. 5.8. Dietary fish oil does not alter the progression of Lyme arthritis (145). Swelling (A) of the soy oil (SO)- and fish oil (FO)-fed mice was assessed using metric calipers. Arthritis severity scores (B), arthritis type scores (B), and Borrelia loads (C) shown are from joints on day 21 post-infection. Values in (A) and $(B)$ are means \pm SEM. In (C), each symbol represents an individual mouse and bars represent the median value. $n=10$ per group. Results are representative of 3 separate experiments all yielding similar results. 
to more definitively determine whether dietary FO substitution had an effect on Lyme arthritis development, we used H \& E-stained joint sections to assess arthritis severity of FO- and SO-fed mice at days 10 and 21 post-infection. We calculated severity scores on a scale of $0-4$, where 0 represents no inflammation and 4 represents severe inflammation, as described previously (244) and in Materials \& Methods. We found no significant difference in arthritis severity in mice fed the FO diet compared to those fed the SO diet (fig. 5.8 B) suggesting that dietary FO substitution does not affect inflammation resulting from $B b$ infection.

Neutrophils are known to mediate the development of Lyme arthritis and are the predominant cell type during severe joint inflammation in this model $(130$, 134). Macrophages tend to predominate later during the infection and correlate with resolution of Lyme arthritis (130). To assess the affect of dietary FO on the joint immune cell infiltrate, we determined arthritis type scores based on $\mathrm{H}$ \& E stained joint sections, as described in Materials \& Methods. A type score of 0 indicates the presence of limited immune cells, a type score of 1 indicates a predominance of macrophages, and a type score of 4 indicates a predominance of neutrophils. We found no differences in the joint immune cell infiltrates in mice fed the FO diet compared to those fed the SO diet (fig. 5.8 B), suggesting that dietary FO substitution does not affect recruitment of neutrophils or macrophages to sites of $B b$ infection.

We hypothesized that the drastic effects of FO on the eicosanoid profile during $B b$ infection would result in a defect in the ability of mice to clear $B b$ 
infection due to attenuated inflammation. Therefore, we isolated DNA from the joints of SO- and FO-fed mice at days 10 and 21 post-infection and used quantitative Real-Time PCR to detect copies of bacterial flagellin (FlaB) normalized to 1000 copies of mouse nidogen (nido). However, just as we saw no differences in arthritis severity or joint immune cell composition, the mice fed the diet in which FO was the primary FA source displayed no defect in their ability to clear $\mathrm{Bb}$ infection when compared to mice fed the $\mathrm{SO}$ diet (fig. 5.8 C).

Together, these data indicate that although dietary FO substitution results in a drastically altered eicosanoid microenvironment, it does not affect the ability of mice to mount an immune response and clear $B b$ from infected joints.

\section{F. Dietary fish oil substitution does not affect Lyme carditis severity, heart immune cell composition, or cardiac $B b$ loads.}

Besides arthritis, carditis is also a significant sequelae to infection with $B b$. To investigate the effect of dietary FO substitution on Lyme carditis, we fed mice the FO or SO diets for 2 weeks prior to infection with $1 \times 10^{5} \mathrm{Bb}$. On days 10 and 21 post-infection, we sagittally bisected hearts and used H \& E stained tissue sections to assess carditis severity and type scores as described in Materials \& Methods. We found no difference in the recruitment of immune cells to the cardiac tissue of FO-fed mice or the ability of these mice to develop carditis when compared to SO-fed mice (fig. 5.9 A). We also detected no defect in the ability of these mice to clear $B b$ from infected cardiac tissue (fig. 5.9 B). These data indicated that, similar to our findings in joint tissue, dietary FO 
A

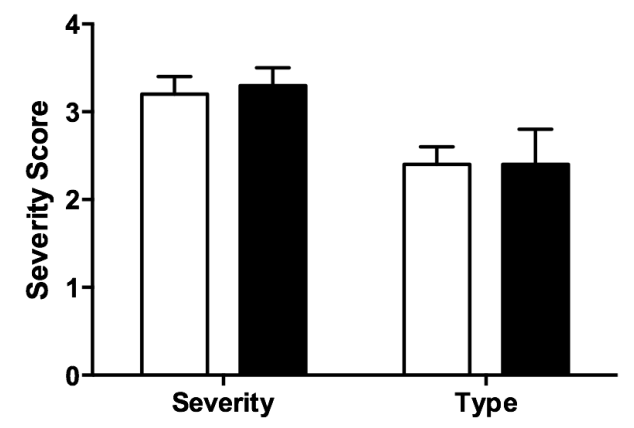

B

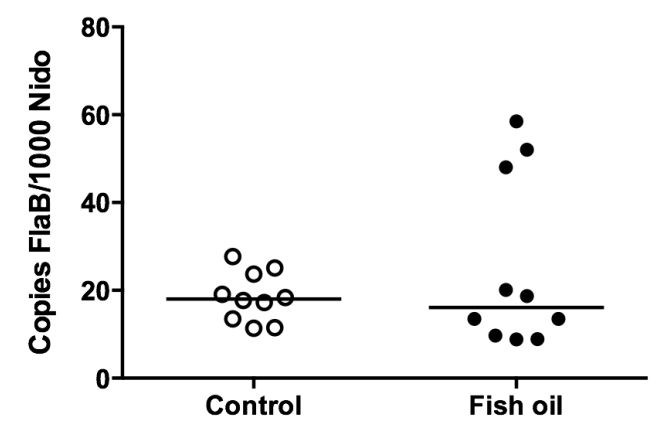

Fig. 5.9. Dietary fish oil substitution does not alter progression of Lyme carditis (145). Carditis severity and type scores (A) of mice fed a soy oil (SO) or fish oil (FO) diet on day 21 post-infection. Values are mean \pm SEM. (B) Borrelia burgdorferi DNA detected in heart tissue of SO- or FO-fed mice on day 21 postinfection. Each symbol represents an individual mouse and bars indicate median values. $n=10$ per group and data is representative of three separate experiment with similar results. 
substitution does not diminish the ability of mice to mount an immune response and clear $B b$ from infected cardiac tissue.

\section{DISCUSSION}

Lyme disease is the most common vector-borne disease in the United States and is endemic in Europe and Asia (120). It is caused by infection with the spirochete Borrelia burgdorferi $(B b)(245)$. Lyme disease is treatable with antibiotics such as doxycycline, although the treatment window is narrow and must take place early on during infection. As symptoms are ambiguous (flu-like) and frequently overlooked, infection often goes undiagnosed. If left untreated, disease progresses to a severe arthritis that localizes to the large joints including the knee (222). Cardiovascular and possibly neurological disorders may also arise.

Dietary fish oil (FO) consumption has been shown to be beneficial for the alleviation of symptoms arising from cardiovascular disease, diabetes, cystic fibrosis, rheumatoid arthritis, and cancer $(81,210-214)$. However, the exact mechanisms by which FO mediates its anti-inflammatory actions are unclear. Previous work suggests that the major $\omega-3$ fatty acids (FA) present in $\mathrm{FO}$ are able to efficiently out-compete $\omega-6$ FA, such as arachidonic acid (AA), for incorporation into membrane phospholipids, making them the preferential substrate for metabolism via cyclooxygenase (COX) and lipoxygenase (LOX) enzymes (215-217). As the metabolism of FO-derived $\omega-3$ FA [eicosapentaenoic acid (EPA) and docosahexaenoic acid (DHA)] results in the production of less 
bioactive eicosanoids [the 3-series prostaglandins (PG) and 5-series leukotrienes $(\mathrm{LT})$ ], it would follow that the resulting eicosanoid microenvironment would have diminished inflammatory potential. Furthermore, FO-derived DHA is known to directly inhibit the enzyme COX-2 $(233,234)$, which is responsible for the production of prostaglandins that are typically believed to have pro-inflammatory functions, such as $\mathrm{PGE}_{2}$. Recent data has even suggested that FO-derived $\omega-3$ FA are able to directly influence inflammation via targeted inhibition of important immune signaling pathways. DHA suppresses inducible nitric oxide synthase (iNOS) expression and nitric oxide (NO) synthesis in murine peritoneal macrophages and RAW264 cells via inhibition of NF-KB activation and upregulation of intracellular glutathione (GSH) (220). EPA and DHA are able to prevent inflammasome-driven inflammation via inhibition of NLRP3 and subsequent caspase-1 activation and IL-1 $\beta$ secretion (221). Taken together, these studies suggest that dietary FO is able to dampen inflammation through a variety of direct and indirect mechanisms.

The murine model of experimental Lyme disease recapitulates very well the progression of human Lyme disease and allows the study of both arthritis and carditis symptoms. To investigate the effects of dietary FO on Lyme arthritis and carditis, we fed mice a diet in which the main FA source was from Menhaden fish oil, as opposed to soy oil in the control diet. The main FAs in FO are EPA and DHA, while the main FAs in SO are AA and linoleic acid (LA). Mice were fed these diets for two weeks prior to being infected with $B b$, as well as throughout the entire course of infection. Disease was monitored throughout the course of 
21 days and mice were sacrificed on days 10 and 21 post-infection in order to collect tissue samples for assessment of disease severity, immune cell composition, and eicosanoid production at these time points. The hypothesis of this study was that mice fed the FO diet would have an altered eicosanoid profile that was more anti-inflammatory in nature compared to the mice fed the SO diet, leading to attenuated Lyme arthritis and carditis. Furthermore, we hypothesized that the reduced capacity of these mice to produce robust inflammation would lead to a defect in their ability to effectively clear infection from these tissues.

Indeed, dietary FO substitution resulted in a dramatic alteration of the eicosanoid profile. Whereas the SO-fed mice produced mainly AA- and LAderived eicosanoids, their production was dramatically reduced in the mice fed the FO diet. EPA- and DHA-derived eicosanoids, however, were abundant in the FO-fed mice. In fact, dietary FO substitution resulted in compositional changes to the eicosanoid profile in every eicosanoid subclass (fig. 5.2). Although the majority of changes to the eicosanoid profile resulting from dietary FO substitution were compositional in that the eicosanoids being produced were from EPA or DHA origin (as opposed to AA or LA origin), the production of AAderived metabolites was also altered. In SO-fed mice, $\mathrm{PGE}_{2}$ was the most abundant COX-2 eicosanoid produced. However, in FO-fed mice, production of $\mathrm{PGE}_{2}$ was drastically reduced and the production of the $\mathrm{PGD}_{2}$ dehydration product $15 d-P G J_{2}$ was increased. Interestingly, the levels to which $15 d-P G J_{2}$ was produced in the FO-fed mice was comparable to the levels at which $\mathrm{PGE}_{2}$ 
was produced in the SO-fed mice, suggesting that dietary FO led to a "redirection" of $A A$ metabolism from $\mathrm{PGE}_{2}$ synthesis to $\mathrm{PGD}_{2}$ synthesis.

The fact that the majority of eicosanoids produced in the FO-fed mice were derived from EPA and DHA and thus less bioactive and that even the few abundant AA-derived eicosanoids produced were anti-inflammatory in nature suggested that the overall tone of the eicosanoid microenvironment in the FO-fed mice was anti-inflammatory. However, when we assessed Lyme arthritis and carditis, we found that inflammation in the FO-fed mice was comparable in severity and type to that of SO-fed mice. Furthermore, the ability of FO-fed mice to clear $B b$ from both ankle and heart tissues was equivalent to the SO-fed mice. These data suggest that although the eicosanoid profile in the FO-fed mice was dramatically altered compositionally, it did not affect the ability of these mice to mount an effective immune response and clear infection with $B b$.

It is important to note that the levels of EPA and DHA provided in our experimental diet were 10 - to 20 -fold what is possible in humans. Furthermore, the rates and abilities of different FA to be incorporated into mouse and human membranes can differ dramatically (246) and may also depend on source [e.g.: krill oil vs. fish oil (247)]. Thus, the fact that dietary FO substitution resulted in such a dramatic shift in the composition of the eicosanoid profile of mice infected with $B b$ likely does not accurately portray what would occur in humans ingesting reasonable amounts of dietary FO for the purpose of dampening inflammation. While $\omega$-3 FA-derived eicosanoids are less bioactive than their $\omega-6$ counterparts, it could be that the excessive amount of $\omega-3$ FA provided in our FO diet allowed 
the production of such high levels of FO-derived eicosanoids that an "inflammatory threshold" was reached allowing $B b$-induced arthritis and carditis to take place. For example, although AA-derived $\mathrm{LTB}_{4}$ is a much more potent neutrophil chemoattractant and has a higher association constant for binding with high-affinity receptors than EPA-derived $\mathrm{LTB}_{5}, \mathrm{LTB}_{4}$ and $\mathrm{LTB}_{5}$ are equally potent at inducing neutrophil degranulation and binding low-affinity receptors (217). Even though AA-derived eicosanoids are much more potent than EPA- or DHAderived eicosanoids, it is possible that in the FO-fed mice, EPA- and DHAderived eicosanoids reached levels sufficient to induce an immune response. The fact that we saw no differences in the severity of inflammation or recruitment of immune cells to the sights of infection supports this hypothesis.

Another possible explanation for the fact that dietary FO substitution did not dampen inflammation resulting from infection with $B b$ is the fact that experimental Lyme disease is not regulated by the NLRP3 inflammasome or iNOS, two immune mechanisms inhibited by FO-derived FA. While Lyme arthritis is dependent on caspase- 1 cleavage and IL- $1 \beta$ production, this process is independent of NLRP3 and is instead mediated by the TLR-2/MyD88 axis (248). While iNOS mRNA expression is up-regulated in response to infection with $B b$ in dogs (249), iNOS-deficient mice have no defect in their ability to develop disease, indicating that iNOS is dispensable for the development of Lyme arthritis (250). Interestingly, in a mouse model of colitis it was found that dietary FO supplementation led to an increase in the production of the chemokine KC (251) which is responsible for the development of Lyme arthritis 
and carditis (134). Therefore, it is possible that total KC production was able to reach levels enabling the recruitment of neutrophils to sites of infection and subsequent development of disease despite an overall anti-inflammatory eicosanoid microenvironment in the FO-fed mice.

This study demonstrates the complexities that exist in understanding the role of eicosanoids in inflammation. While FO-derived eicosanoids are less bioactive than their $\omega-6$ FA-derived counterparts, it is likely that a threshold exists after which these eicosanoids can, too, become pro-inflammatory. Similar to many nutritional supplements, it is likely that the effects of FO are only beneficial when taken in moderation. Furthermore, although FO supplementation has been shown to be beneficial for the alleviation of rheumatoid arthritis, the fact that we saw no difference in Lyme arthritis between FO- and SO-fed mice exemplifies the differences that exist in types of inflammation and variables that determine capable therapeutic intervention even within different types of arthritis. 


\section{CHAPTER SIX}

\section{DYNAMIC EICOSANOID PRODUCTION DURING AUTOANTIBODY-DRIVEN K/BXN SERUM-TRANSFER ARTHRITIS}

\section{INTRODUCTION}

The K/BxN model of arthritis was described in 1996 by Kouskoff, et al. (252) and is the prototypical model for arthritides that are regulated by autoantibodies and immune complexes. It is widely used in an effort to delineate mechanisms that may regulate human Rheumatoid arthritis (RA) due to the many similarities that exist between the two arthritides including synoviocyte proliferation, synovitis, leukocyte invasion, pannus formation, erosion of cartilage and bone, polyclonal B cell activation, hypergammaglobulinemia, and autoantibody production $(252,253)$.

$\mathrm{K} / \mathrm{BxN}$ mice are generated when mice transgenic for the KRN $\mathrm{T}$ cell receptor (TCR), which recognizes the bovine ribonuclease peptide RNase 42-56 presented by I-A ${ }^{k}$ MHC II, are crossed with NOD mice. Inflammation develops due to the KRN TCR aberrantly recognizing a peptide derived from the ubiquitously expressed glucose-6-phosphate isomerase (GPI) presented by NOD $A^{97}$ MHC II. K/BxN mice develop severe, chronic polyarthritis around 4-5 weeks of age. Arthritis in K/BxN mice is fully dependent on the transgenic KRN TCR and the breaking of tolerance leading to production of autoantibodies recognizing GPI $(253,254)$. GPI autoantibody effector function in $\mathrm{K} / \mathrm{BxN}$ mice is regulated by 
the alternative pathway of complement, most notably C5a (255-257). The localization of inflammation to joint tissue despite recognition of a ubiquitously expressed peptide is believed to be due to the lack of cell membrane-bound complement inactivators such as decay-accelerating factor (DAF/CD55) and membrane cofactor of proteolysis (MCP/CD46) expressed on cartilage. Localization of inflammation to the joint may further be explained by the formation of complement factor 3 (C3)-lgG (anti-GPI) immune complexes (IC) that develop on the cartilage surface, resulting in the activation of the alternative complement pathway and subsequent cleavage of C5a from C5 (255).

The transfer of serum containing anti-GPI autoantibodies from K/BxN mice to wild-type recipient mice leads to the development of a transient arthritis, in which severity and duration correlates with the presence and eventual degradation of anti-GPI autoantibodies present in the passaged serum. This model allows for the study of effector mechanisms that regulate arthritis independent of the breaking of tolerance in $\mathrm{K} / \mathrm{BxN}$ mice. Inflammation resulting from the transfer of $\mathrm{K} / \mathrm{BxN}$ serum is independent of adaptive immunity and is dependent on innate immune cells, most notably PMN, which are indispensible for the development of disease $(163,164,258)$.

Eicosanoids, a major component of the innate immune compartment, have been shown to play regulatory roles in the development of $\mathrm{K} / \mathrm{BxN}$ serum-transfer arthritis (KSTA). Cyclooxygenase-1 (COX-1)-derived prostacyclin ( $\left.\mathrm{PGI}_{2}\right)$ signaling through its cognate receptor, IP, is required for both the development 
and maintenance of arthritis in this model, while COX-2-derived prostaglandins appear dispensable (259).

5-lipoxygenase (5-LOX)-derived leukotriene $B_{4}\left(L_{T B}\right)$ plays numerous roles in the development of KSTA. In order for $\mathrm{LTB}_{4}$ to be produced, 5-LOX must translocate to the nuclear envelope and associate with 5-LOX activating protein (FLAP). This complex metabolizes arachidonic acid (AA) into leukotriene $A_{4}$ $\left(\mathrm{LTA}_{4}\right)$, which is metabolized into $\mathrm{LTB}_{4}$ by $\mathrm{LTA}_{4}$ hydrolase $\left(\mathrm{LTA} \mathrm{A}_{4} \mathrm{H}\right)$. Approximately $20 \%$ of in vivo $\mathrm{LTB}_{4}$ production results from transcellular biosynthesis, in which $\mathrm{LTA}_{4}$ is secreted by 5 -LOX-expressing cells (bone marrowderived myeloid cells) and metabolized by neighboring $\mathrm{LTA}_{4} \mathrm{H}$-expressing cells (260). In 2010, Chen et al. observed that transcellular biosynthesis of $\mathrm{LTB}_{4}$ plays a significant role in the development and maintenance of KSTA (110). It was found that radio-resistant (bone marrow-derived) cells, most likely PMN, produce $\mathrm{LTA}_{4}$, which is metabolized by $\mathrm{LTA}_{4} \mathrm{H}$-expressing fibroblast-like synoviocytes (FLS) into $\mathrm{LTB}_{4}$. In this study, $\mathrm{LTB}_{4}$, acting through its cognate receptor BLT-1, stimulated FLS invasion and migration, as well as FLS-mediated erosive pannus formation (110).

Further studies have implicated $\mathrm{LTB}_{4}$ as part of a "lipid-cytokinechemokine cascade" that mediates multiple "waves" of PMN recruitment and subsequent development of KSTA (261). These studies suggest that following passage of $\mathrm{K} / \mathrm{BxN}$ serum, C5a signals through $\mathrm{C} 5 \mathrm{aR}$ on a "first wave" of PMN and induces the production of $\mathrm{LTB}_{4}$ by these cells. $\mathrm{LTB}_{4}$ produced by the "first wave" of PMN signals through BLT-1 to recruit a "second wave" of PMN, which 
are activated by immune complex (IC) signaling through the FcyRIII, leading to the production of IL-1 $1 \beta$. This "second wave" of PMN participate in their own recruitment both directly, by expressing the chemokines CXCL2 and CCL3, and indirectly via production of $\mathrm{IL}-1 \beta$, which induces the production of CXCL1, CXCL5, CCL4, and CCL9 by joint structural cells $(261,262)$. This rapid and massive accumulation of cytokine- and $\mathrm{LTB}_{4}$-producing PMN at the cartilage surface results in a destructive arthritis due to the stimulation of local proinflammatory cytokine production and FLS invasion, migration, and erosive pannus formation (110). While the majority of studies suggest that the $\mathrm{LTB}_{4} / \mathrm{BLT}-1$ axis is the main signaling pathway by which $\mathrm{LTB}_{4}$ mediates its activity, in a study using $\mathrm{LTB}_{4} \mathrm{R}$ knock-out mice $\left(\mathrm{BLT}-1^{-1-}\right.$ and $\left.\mathrm{BLT}-2^{-1-}\right)$, it was found that BLT-1 and BLT-2 both play significant, yet non-redundant roles in the development of KTSA (263).

We have previously completed a study in which we assessed the temporal production of 104 distinct eicosanoid species throughout the course of Lyme arthritis (144). In the present study, we utilized the same protocol in order to determine the production of eicosanoids throughout the course of KSTA. The mechanisms by which eicosanoids regulate inflammation in these two models are dissimilar; While COX-1-derived metabolites regulate the development and maintenance of KSTA, COX-2-derived metabolites seem to play a greater role in Lyme arthritis (141). 5-LOX metabolites play a role in the regulation of inflammation in both models, albeit to a lesser extent in Lyme arthritis (142). The results herein serve to demonstrate that although inflammatory arthritides may 
have overlapping disease manifestations and perhaps even similar pathways that converge downstream, the mechanisms that regulate inflammation vary drastically and depend on the original instigating source of inflammation. It is crucial to keep this information in the forefront of the mind when efforts are being made to translate research preformed in murine model systems to the development of therapeutics for human disease.

\section{RESULTS}

\section{A. Passage of $\mathrm{K} / \mathrm{BxN}$ serum to $\mathrm{C} 3 \mathrm{H} / \mathrm{HeJ}$ mice results in severe polyarthritis.}

In order to induce arthritis, we injected 4-6 week old $\mathrm{C} 3 \mathrm{H} / \mathrm{HeJ}$ mice with pooled serum isolated from arthritic $\mathrm{K} / \mathrm{BxN}$ mice and boosted them two days later, as described (264). We monitored disease throughout the course of arthritis by determining clinical scores based on front on hind paw edema, as described in Monach, et al. 2008 (264), and by measuring dorsovental swelling of hind joints using metric calipers (fig. 6.1 A, B). Injection of K/BxN serum resulted in progressive paw swelling, involving both front and hind paws, that peaked at day 10 post-serum transfer. On days $3,7,14,17$, and 21 post-serum transfer, we collected joint tissue from arthritic mice and compared histologicallydetermined arthritis severity and type scores to control mice that had been injected with normal mouse serum (NMS) (fig. 6.1 C, D). Arthritis severity correlated with levels of paw edema and suggested that the peak of arthritis occurred between days 7 and 14 post-serum transfer. Arthritis type scores are generated in an effort to determine cell types present in joint tissue. 
A

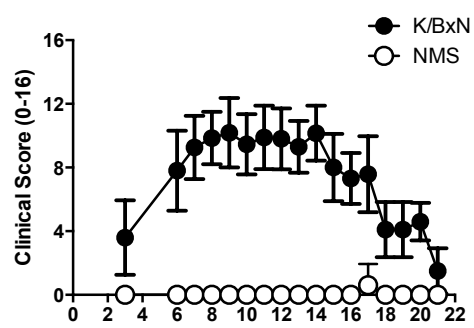

Day Post-Serum Transfer

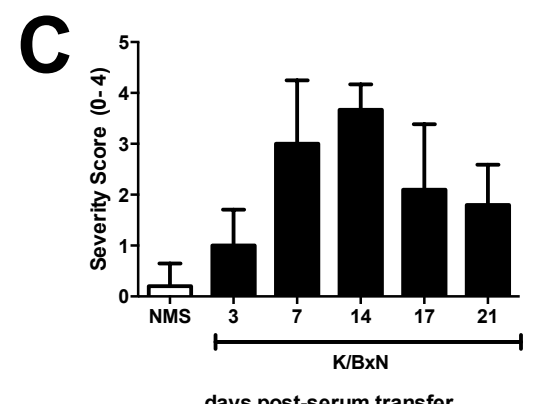

B

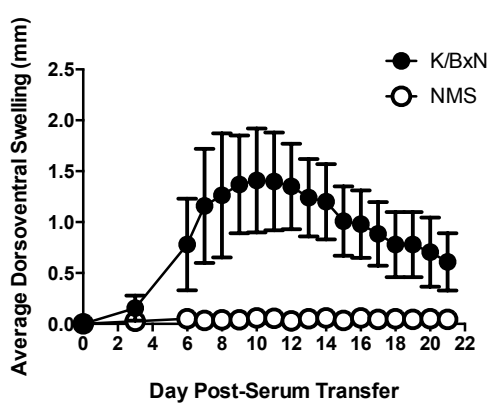

D

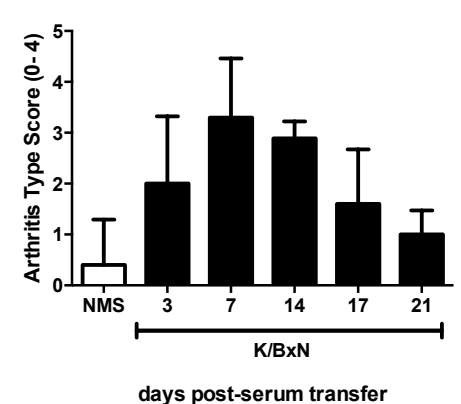

Fig. 6.1. Severe arthritis develops in response to passage of $K / B \times N$ serum to $\mathrm{C} 3 \mathrm{H} / \mathrm{HeJ}$ mice. $\mathrm{K} / \mathrm{BxN}$ or normal mouse serum was injected intraperitoneally (i.p.) into $\mathrm{C} 3 \mathrm{H} / \mathrm{HeJ}$ mice and disease was allowed to progress for 21 days. Clinical scores (A) were determined as described by Monach, et al. 2008 (264), and represent the sum of paw scores rated on a 1-4 scale. Swelling (B) was determined using metric calipers and by subtracting day 0 values from subsequent measurements. Jennifer Hughes-Hanks generated Severity (C) and type (D) scores by histological assessment of $\mathrm{H}$ \& E-stained joint sections. Values represent mean \pm SD. 
Lower type scores indicate a predominance of macrophages in joint tissue, while higher type scores indicate a predominance of neutrophils. We found that the highest type scores were observed on days 3 through 17 post-serum transfer, suggesting neutrophils were the dominant cell type in arthritic lesions at these time points. The dominance of neutrophils during the peak of $\mathrm{K} / \mathrm{BxN}$ serum transfer arthritis (KSTA) corroborates data implicating neutrophils as mediators of inflammation in this model $(163,164,258)$.

\section{B. Eicosanoid enzymatic pathways are activated during KSTA.}

On days $3,7,14,17$, and 21 post-serum transfer, we extracted lipids from joint tissue and quantified eicosanoids using liquid chromatography tandem mass spectrometry (LC-MS/MS) as previously described (144). This allowed us to determine the production of eicosanoids throughout the course of KSTA. Using this information, we generated a heat map displaying the relative fold change of eicosanoids compared to production in control, NMS-treated mice (fig. 6.2). This data indicated that, throughout the course of KSTA, arachidonic acid metabolites were dynamically affected.

To better understand the enzymatic pathways that were being activated in response to the passage of $\mathrm{K} / \mathrm{BxN}$ serum, we assessed enzyme usage based on the fold change data displayed on the heat map. We determined the percent of metabolites that were affected, positively or negatively, in each pathway in response to transfer of $\mathrm{K} / \mathrm{BxN}$ serum. This allowed us to assess both total enzyme usage (fig. 6.3 A) and temporal enzyme usage (fig. 6.3 B), suggesting 

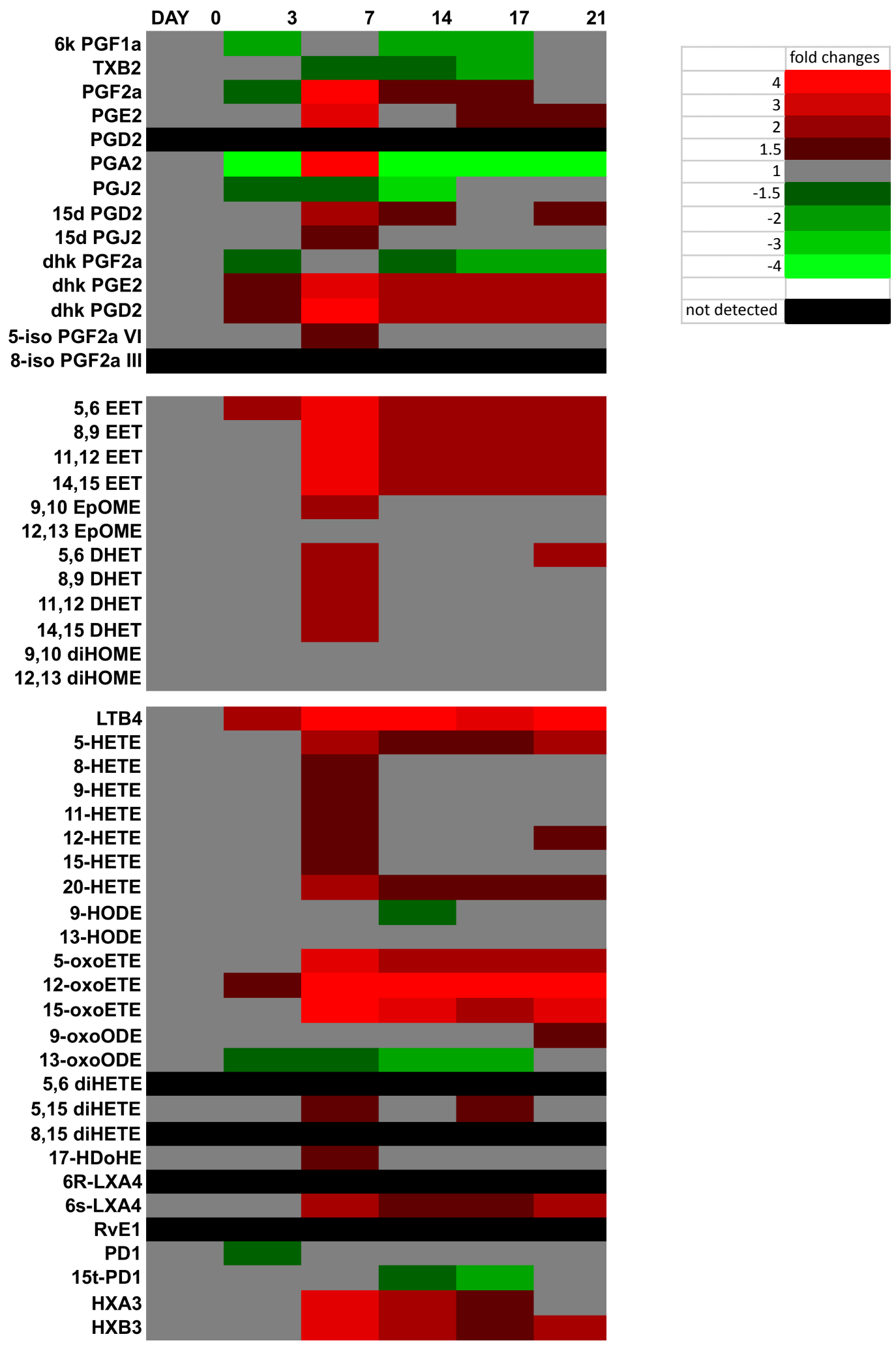
Fig. 6.2. Joint eicosanoids are dynamically affected by passage of $K / B x N$ serum. Heat map displays the relative fold change of select AA-derived eicosanoids in the joints of mice on days $3,7,14,17$, and 21 post-K/BxN serum transfer compared to expression in the joints of mice passaged with normal mouse serum (NMS, "day 0"). For eicosanoids that were ND on day 0 , the relative fold change was determined from the day that they first were detected. ND (black) indicates that an eicosanoid was either not detected or was detected in concentrations below the limit of detection. $n=5$ on day 0,9 on day 3 and 14 , 10 on day 7 and 21 , and 8 on day 17 post-serum transfer. (LC-MS/MS analysis and composition of heat map by Dr. Darren S. Dumlao.) 
A

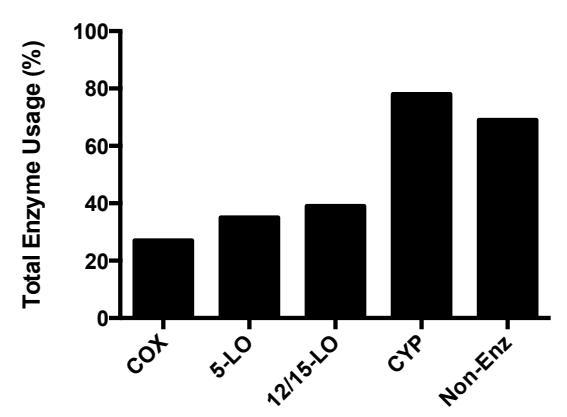

B

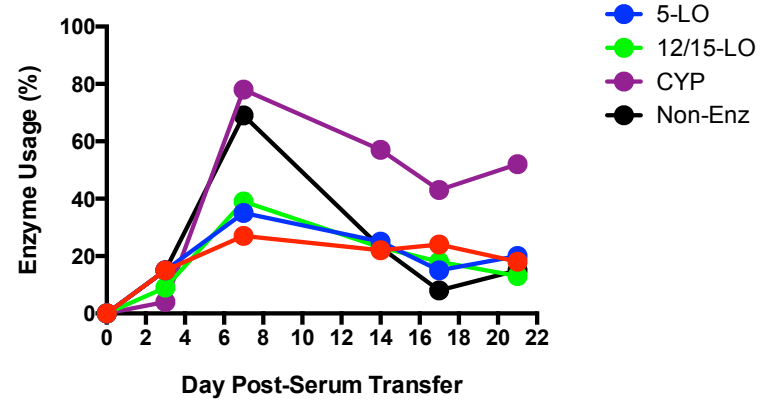

Fig. 6.3. Eicosanoid enzymatic pathways engaged during KSTA. Enzymatic pathway usage was determined by calculating the percentage of metabolites that were affected by a fold change of 1 or more in a given pathway at any given time (A). Temporal enzyme usage was determined by calculating the percentage of metabolites affected by a fold change of 1 or more in a given pathway on days 3 , 7, 14, 17, and 21 post-serum transfer (B). (LC-MS/MS analysis performed by Dr. Darren S. Dumlao, calculation of enzyme usage performed by Anna M. Cunningham.) 
which pathways were affected during KSTA. The majority of metabolites that were affected were generated either non-enzymatically or via the cytochrome P450 (CYP) pathway, suggesting that metabolites produced through these pathways are important mediators of KSTA.

\section{Dynamic regulation of Cytochrome P450 metabolites during KSTA.}

The CYP pathway is responsible for the production of two major groups of vasoactive eicosanoids, the hydroxyeicosatetraenoic acids (HETEs) and the epoxyeicosatetraenoic acids (EETs). For the most part, the HETEs (e.g.: 20HETE) are vasoconstrictive and the EETs (e.g.: 11,12-EET) are vasodilatory (265). In general, these activities are mediated via regulation of calciumdependent potassium channels (266-268). The CYP pathway has been implicated to correlate in some instances with arthritis, as hepatic levels of CYP subfamily enzymes were elevated in a rat model of adjuvant-induced arthritis (269) and a study of Taiwanese Rheumatoid arthritis patients noted that a polymorphism in the human gene CYPIAl correlated with resistance to disease (270-273).

We observed that the CYP pathway was dynamically affected during KSTA (fig. 6.3 A, B) and thus examined more closely the production of the CYP metabolites 16-HETE, 18-HETE, 19-HETE, and 20-HETE (fig. 6.4 A-D). While CYP metabolites are, for the most part, accepted to be vasoconstrictive, 16HETE, 18-HETE, and 19-HETE have been observed to have vasodilatory functions (265). These discrepancies may be explained by the fact that the 

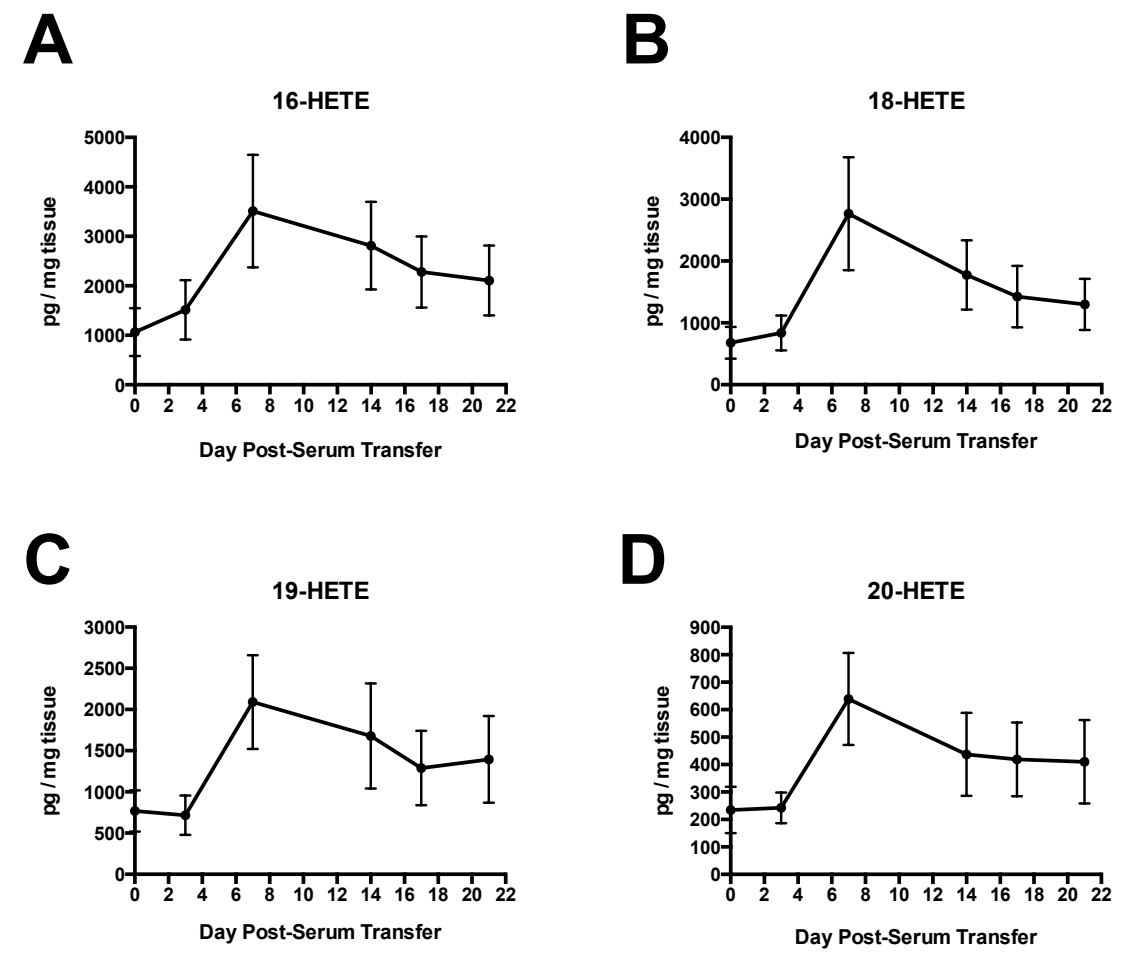

Fig. 6.4. Cytochrome P450-derived hydroxyeicosatetraenoic acids (HETEs) expression in joints of arthritic mice. Cytochrome P450 (CYP)-derived HETEs were quantified in the joints of mice that had received either $\mathrm{K} / \mathrm{BxN}$ or normal mouse serum using LC-MS/MS on days $0,3,7,14,17$, and 21 posttransfer. Values are means $\pm \mathrm{SD}, n=5$ on day 0,9 on day 3 and 14,10 on day 7 and 21, and 8 on day 17 post-serum transfer. (Values by Dr. Darren S. Dumlao, figures by Anna M. Cunningham.) 
function of these molecules appears to be tissue-specific and may vary depending on the vascular bed (274). 20-HETE, however, has been shown in numerous studies to be a reliable and potent vasoconstrictive metabolite (266). In fact, increased 20-HETE expression resulting from inhibition of cyclooxygenase-2 (COX-2) has been found to be responsible for the increased risk of heart attack associated with the drug Vioxx ${ }^{\circledR}$ (rofecoxib) (275). The expression of 16-HETE, 18-HETE, 19-HETE, and 20-HETE peaked concomitantly at day 7 post-serum transfer (fig. 6.4 A-D), likely playing a role in regulating the influx of immune cells to the site of inflammation.

CYP-derived EETs are potent vasodilators, but have also been shown to play a role in mitogenesis and the production of hormones such as growth factors and vasopressin (276-278). These molecules are also anti-inflammatory as they inhibit the expression of adhesion molecules on endothelial cells (279). We found that, similar to the CYP-derived HETEs, the expression of 5,6-EET, 8,9EET, 11,12-EET, and 14,15-EET peaked on day 7 post-serum transfer (fig. 6.5 A-D). As these molecules are potent vasodilators and mitogens, it is likely that at these time points they are upregulated to allow for tissue infiltration of immune cells and to induce synoviocyte proliferation. Although their expression appears to wane slightly at later time points, the expression of these metabolites remains higher than the HETEs throughout the course of KSTA (fig. 6.5). This suggests that the balance of vasodilatory and vasoconstrictive metabolites is tipped toward the former, allowing vascular permeability for the influx of immune cells. Furthermore, as these metabolites have anti-inflammatory activity, their 
A

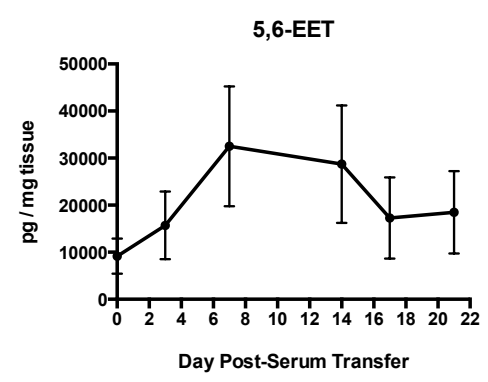

C

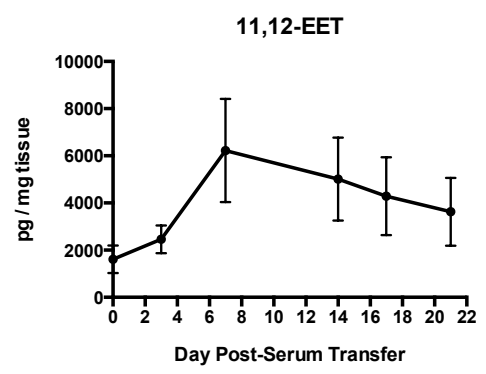

B

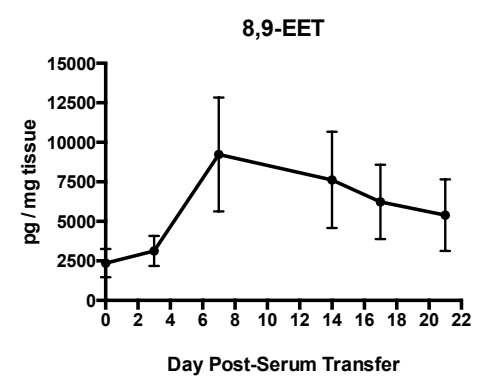

D

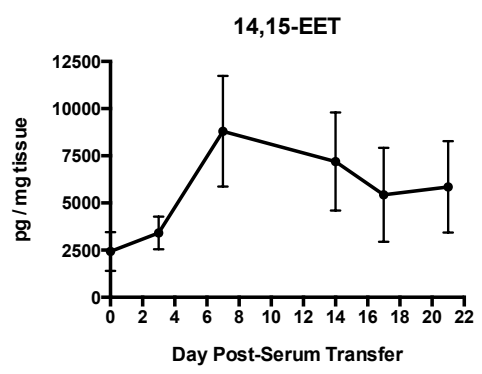

Fig. 6.5. Cytochrome P450-derived epoxyeicosatetraenoic acids (EETs) are produced in the joints of arthritic mice. Cytochrome P450 (CYP)-derived EETs were quantified in the joints of mice that had received either $\mathrm{K} / \mathrm{BxN}$ or normal mouse serum using LC-MS/MS on days $0,3,7,14,17$, and 21 posttransfer. Values are means $\pm \mathrm{SD}, n=5$ on day 0,9 on day 3 and 14,10 on day 7 and 21, and 8 on day 17 post-serum transfer. (Values by Dr. Darren S. Dumlao, figures by Anna M. Cunningham.) 
expression at later time points could serve to limit inflammation and induce resolution.

\section{Expression of non-enzymatically generated eicosanoids during KSTA.}

Non-enzymatic metabolite production is a reliable indicator of a microenvironment with high oxidative stress (280). Two notable metabolites produced via non-enzymatic mechanisms, 5-iso $\mathrm{PGF}_{2 \alpha}$ IV and 8-iso $\mathrm{PGF}_{2 \alpha}$ III, are elevated in Rheumatic diseases (281) and in certain instances may exert vasoconstrictive activity (282). We measured the production of these metabolites and found that although 5-iso $\mathrm{PGF}_{2 \alpha}$ IV remained unchanged throughout the course of KSTA, 8-iso PGF $_{2 \alpha}$ III was upregulated in response to the transfer of arthritic $\mathrm{K} / \mathrm{BxN}$ serum, peaked at day 7 post-serum transfer, and remained elevated throughout the course of disease (fig. 6.6 A, B). As this molecule has vasoconstrictive activity, its upregulation at day 7 and continued elevation throughout the remainder of disease could suggest that it acts as a counterregulator to the highly expressed vasodilatory EETs, functioning to limit further vascular leakage and immune cell infiltration and induce the resolution of inflammation.

As the non-enzymatic pathway of metabolite generation is highly activated in KSTA (fig. 6.3), we assessed the production of other non-enzymatically produced metabolites including 9-HETE, 8-HDoHE, 16-HDoHe, and 20-HDoHe

(fig. 6.6 C-F). The expression of these metabolites was elevated throughout the course of disease, but peaked at day 7 post-serum transfer. The fact that these 

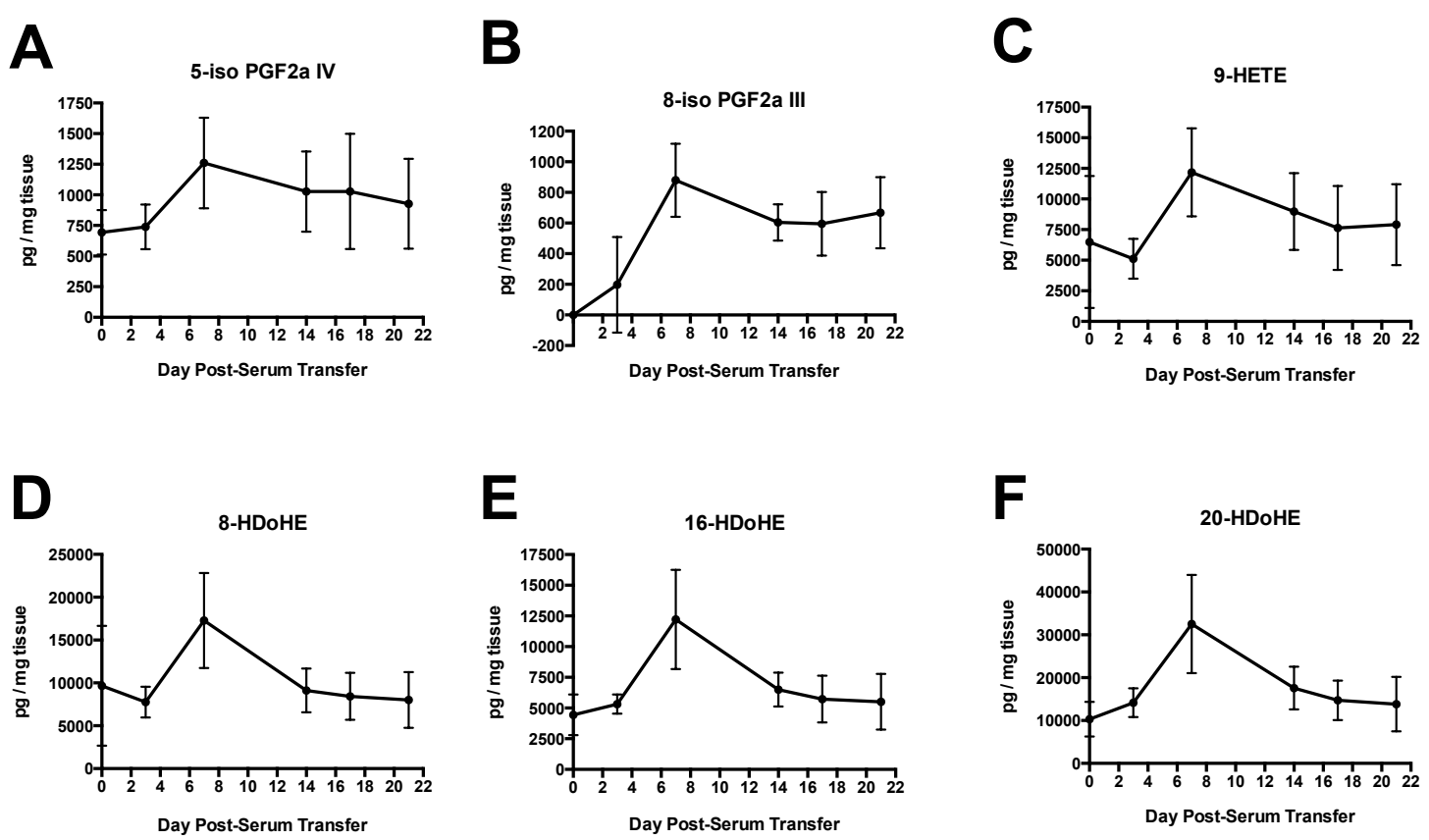

Fig. 6.6. Non-enzymatic eicosanoid production in joints of arthritic mice. Non-enzymatically produced eicosanoids were quantified in the joints of mice that had received either $\mathrm{K} / \mathrm{BxN}$ or normal mouse serum using LC-MS/MS on days $0,3,7,14,17$, and 21 post-transfer. Values are means $\pm S D, n=5$ on day 0,9 on day 3 and 14,10 on day 7 and 21 , and 8 on day 17 post-serum transfer. (Values by Dr. Darren S. Dumlao, figures by Anna M. Cunningham.) 
enzymes were produced to such high levels throughout the course of disease indicates that the microenvironment generated in response to the transfer of arthritic $\mathrm{K} / \mathrm{BxN}$ serum is highly oxidative. This data is in agreement with a study demonstrating that oxidative stress mediates the severity of arthritis in this model, as mice lacking heme oxygenase-1 (HO-1), a potent anti-oxidative stress defense molecule, develop exaggerated arthritis severity (283).

\section{E. Cyclooxygenase metabolite production during KSTA.}

Cyclooxygenase (COX) enzymes (COX-1 and COX-2) are known to have a variety of homeostatic and immune-modulating functions $(60,83,140,141$, 143, 284-287). COX-1 is expressed constituitively in most tissues, while COX-2 is upregulated in response to stimuli such as inflammation. This pattern of expression has led to the hypothesis that COX-1 functions as a housekeeping enzyme while COX-2 and its metabolites are mainly involved in inflammation. However, this is not always the case as COX-1 is known to have functions in immunity $(143,259)$ and COX-2 is involved in gut and kidney homeostatic maintenance $(288,289)$. In the $\mathrm{K} / \mathrm{BxN}$ serum transfer model, COX-1 and its metabolite $\mathrm{PGI}_{2}$ are important for the development and maintenance of arthritis while COX-2 and its metabolite $\mathrm{PGE}_{2}$ are dispensable (259).

We measured the production of COX-1- and COX-2-derived eicosanoids throughout the course of KSTA (fig. 6.7). We found that endogenous (NMStreated) levels of 6-keto-PGF ${ }_{1 \alpha}\left(6 k-P F_{1 \alpha}\right)$, the stable breakdown product of $\mathrm{PGI}_{2}$, were elevated but decreased upon transfer of arthritic K/BxN serum. 

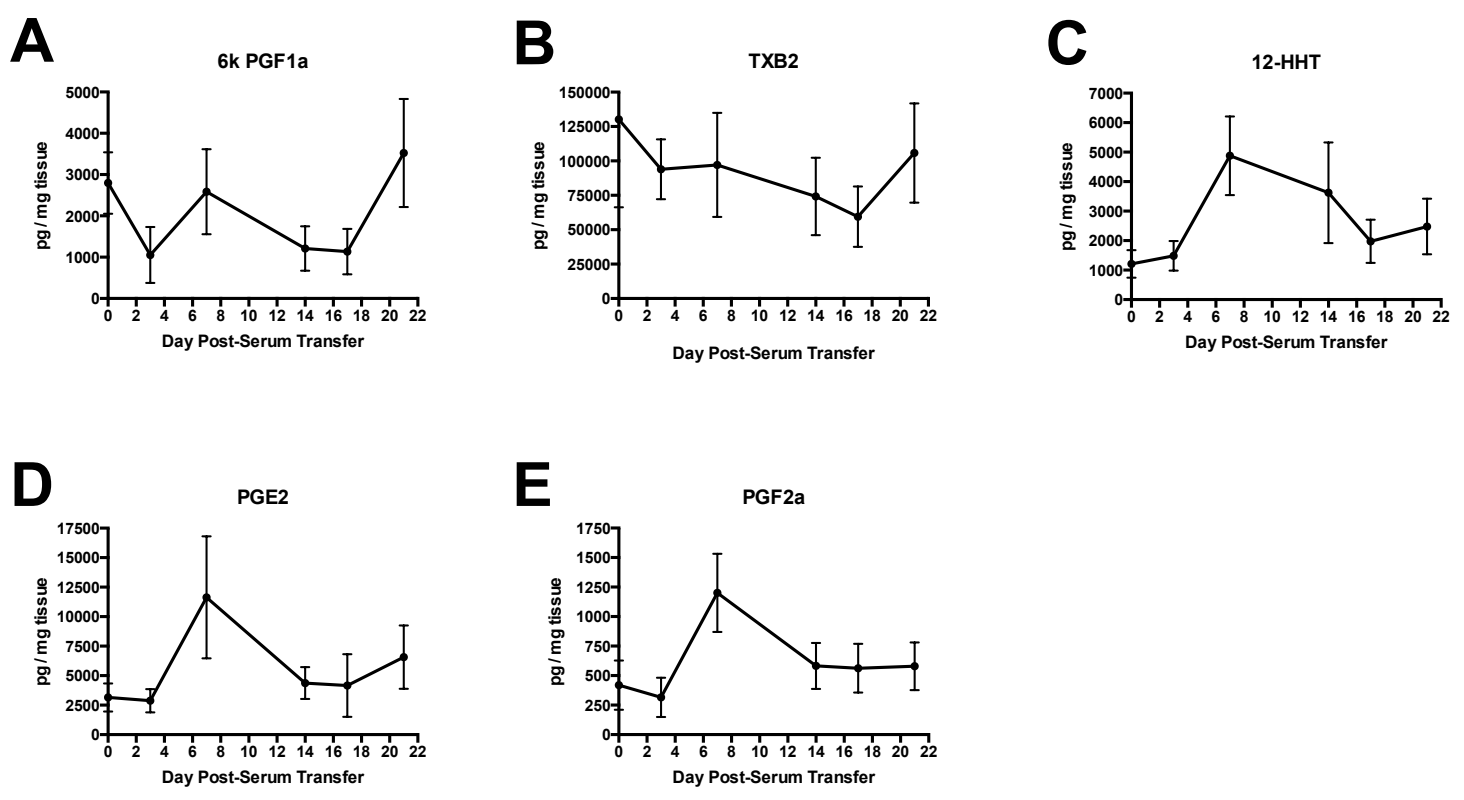

Fig. 6.7. Cyclooxygenase (COX)-derived metabolite production in the joints of arthritic mice. Cyclooxygenase (COX)-derived metabolites were quantified in the joints of mice that had received either $\mathrm{K} / \mathrm{BxN}$ or normal mouse serum using LC-MS/MS on days $0,3,7,14,17$, and 21 post-transfer. Values are means \pm SD, $n=5$ on day 0,9 on day 3 and 14,10 on day 7 and 21 , and 8 on day 17 post-serum transfer. (Values by Dr. Darren S. Dumlao, figures constructed by Anna M. Cunningham.) 
However, levels of this metabolite elevated to endogenous levels on day 7 post serum-transfer, decreased again on days 14 and 17, and became elevated once again on day 21 (fig $6.7 \mathrm{~A}$ ). $\mathrm{PGI}_{2}$ is a potent vasodilator that is found at high levels in the synovial fluid of Rheumatoid arthritis patients (193). In a model of collagen-induced arthritis (CIA), the production of $P \mathrm{PI}_{2}$ is induced when IL-1 $\beta$ stimulates the production of IL-6 by synovial fibroblasts (290). As IL-1 $\beta$ is believed to mediate a "second wave" of PMN in KSTA $(261,291)$, it is likely that the detection of $\mathrm{PGF}_{2 \alpha}$ at these time points is representative of the $\mathrm{PGI}_{2}$ that was produced by this "second wave" of PMN or even a "third wave" of PMN that the "second wave" recruited. Thromboxane $B_{2}\left(T X B_{2}\right)$ is the stable breakdown product of the COX-1 metabolite $\mathrm{TXA}_{2}$. $\mathrm{TX}_{2}$ was found at extremely high levels in NMS-treated control mice as well as throughout the course of arthritis in $\mathrm{K} / \mathrm{BxN}$ serum-treated mice (fig. 6.7 B). As $\mathrm{TXA}_{2}$ is a potent vasoconstrictor, it is possible that this molecule was being expressed at such high levels in order to counter the production of the many vasodilatory EETs.

12-hydroxyheptadecatrienoic acid $(12-\mathrm{HHT})$ is a COX-1 derived eicosanoid that is a high affinity ligand for the $\mathrm{LTB}_{4}$ receptor, BLT-2 (292). We found that 12-HHT was increased throughout the course of KSTA, peaking on day 7 post-serum transfer, remaining elevated at day 14, and gradually decreasing to endogenous low levels of expression (fig. 6.7 C). In vitro, 12-HHT signaling through BLT-2 induces PMN chemotaxis and, in vivo, the presence of BLT-2 on bone marrow cells is required for full development of KSTA (263). Although $\mathrm{LTB}_{4}$ signaling through BLT-1 was shown to be responsible for PMN 
recruitment in vitro, it is likely that $12-\mathrm{HHT}$ can function in a redundant fashion in vivo if necessary. High levels of arachidonic acid (AA) $(\geq 10 \mu \mathrm{M})$ are able to inhibit the production of $\mathrm{LTB}_{4}$ by human peripheral blood neutrophils (PBN) by preventing the dimerization of 5-LOX activating protein (FLAP) (293). AA can accumulate to high levels when cells are in inflammatory conditions, thus it is a distinct possibility that during the peak of arthritis, $\mathrm{LTB}_{4}$ production is abrogated and the redundant function of $12-\mathrm{HHT}$ allows signaling through BLT-2 to progress.

Although not absolutely required for the development of KSTA, COX-2derived prostaglandins are highly immuno-regulatory and likely play at least a minor role in the modulation of arthritis severity in this model. We found that $\mathrm{PGE}_{2}$ and $\mathrm{PGF}_{2 \alpha}$ were increased to a peak of expression at day 7 post-serum transfer (fig. 6.7 D, E). Whether $\mathrm{PGE}_{2}$ functions as a pro- or anti-inflammatory mediator in inflammatory arthritis is variable. It has been suggested that although $\mathrm{PGE}_{2}$ may play an inflammatory role, its production is necessary to induce a "switch" from inflammation to resolution (82). In a model of CIA, inhibition of $\mathrm{PGE}_{2}$ production resulted in suppression of arthritis (294). However, in a model of collagen antibody-induced arthritis, which is independent of adaptive immunity, inhibition of $\mathrm{PGE}_{2}$ production resulted in exacerbated arthritis (295). This data may suggest that $\mathrm{PGE}_{2}$ may play an anti-inflammatory role in models of inflammatory arthritis that do not involve adaptive immunity. Therefore, it could be postulated that $\mathrm{PGE}_{2}$ acts as an anti-inflammatory mediator in KSTA and its peak of expression at day 7 post-serum transfer could 
represent an effort to induce mechanisms of resolution (fig. 6.7 D). Inflammation resulting from transfer of $\mathrm{K} / \mathrm{BxN}$ serum to $\mathrm{COX}-2^{-1-}$ mice is indistinguishable from inflammation in WT mice; however, as the resolution of KSTA is mediated by the degradation of anti-GPI antibodies, it is likely that even if resolution mechanisms were not intact, arthritis would subside. Thus, the role of COX-2/PGE 2 in KSTA remains somewhat unclear.

\section{F. Lipoxygenase production during KSTA.}

5-lipoxygenase-derived metabolites are produced at greater quantities by stimulated mononuclear cells isolated from patients with Rheumatoid arthritis when compared to stimulated mononuclear cells isolated from healthy controls (296). 5-LOX ${ }^{-/-}$mice are resistant to the development of KSTA as $\mathrm{LTB}_{4}$ signaling through its receptor, BLT-2, is required for the development of arthritis in this model $(109,263)$. We found that the production of $\mathrm{LTB}_{4}$ was elevated in the joints of mice throughout the course of KSTA (fig. 6.8 A) corroborating its role as a regulator of inflammation in this model. Another metabolite of the 5-LOX pathway, 5-HETE, can induce the migration of neutrophils through epithelial and endothelial barriers (297). We found that 5-HETE expression was modestly increased at day 7 post-serum transfer (fig. 8 B), which may suggest a possible role in the recruitment of neutrophils to sites of inflammation.

12/15-lipoxygenase-deficient (12/15-LOX $\left.{ }^{-/}\right)$mice develop exacerbated arthritis resulting from passage of $\mathrm{K} / \mathrm{BxN}$ serum suggesting that metabolites generated through this pathway function to limit inflammation (298). Inhibition of 

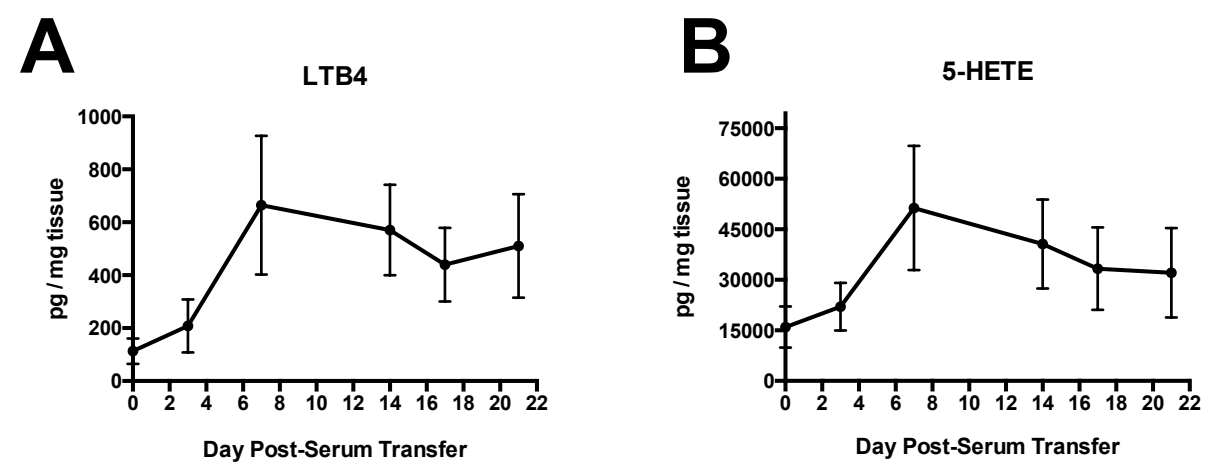

Fig. 6.8. 5-lipoxygenase (5-LOX)-derived metabolite production in the joints of arthritic mice. 5-lipoxygenase (5-LOX)-derived eicosanoids were quantified in the joints of mice that had received either $\mathrm{K} / \mathrm{BxN}$ or normal mouse serum using LC-MS/MS on days $0,3,7,14,17$, and 21 post-transfer. Values are means \pm SD, $n=5$ on day 0,9 on day 3 and 14,10 on day 7 and 21 , and 8 on day 17 post-serum transfer. (Values by Dr. Darren S. Dumlao, figures constructed by Anna M. Cunningham.) 
12/15-LOX correlated with increased activation of p38 MAP kinase, increased production of IL-6, IL-1 $\beta$, IFNy, and $\mathrm{KC}$, and decreased production of the antiinflammatory lipoxin $A_{4}\left(L X A_{4}\right)$ (298). We measured the production of 12-HETE, 15-HETE, $\mathrm{HXA}_{3}, \mathrm{LXA}_{4}$, and PD1, all products of this enzymatic pathway and all of which are implicated to have anti-inflammatory functions (fig. 6.9). We found that while the production of 12-HETE (fig. 6.9 A) and PD1 (fig. 6.9 E) remained relatively unchanged throughout the course of KSTA, 15-HETE (fig. 6.9 B), HXA

(fig. 6.9 C), and $\mathrm{LXA}_{4}$ (fig. 6.9 D) appeared to increase and peak at day 7 postserum transfer. The peak of these anti-inflammatory metabolites at day 7 postserum transfer may corroborate with data suggesting that anti-inflammatory metabolites must be produced early during disease in order for functional resolution to take place at later time points $(7,299)$. 12-HETE, $\mathrm{HXA}_{3}$, and 15HETE were expressed in large quantities, while LXA 4 and PD1 accumulated to only small amounts. However, the quantities to which these metabolites are produced may or may not correlate with their activity, as many of these molecules are extremely potent and can have important functions even when produced in small quantities.

\section{G. KSTA has a higher pro-inflammatory index than Lyme arthritis.}

We calculated inflammatory indices based on the production of pro- and anti-inflammatory eicosanoids throughout the course of KSTA, as described in the supplemental methods of Tam, et al. 2013 (300) (fig. 6.10 A). Our data 

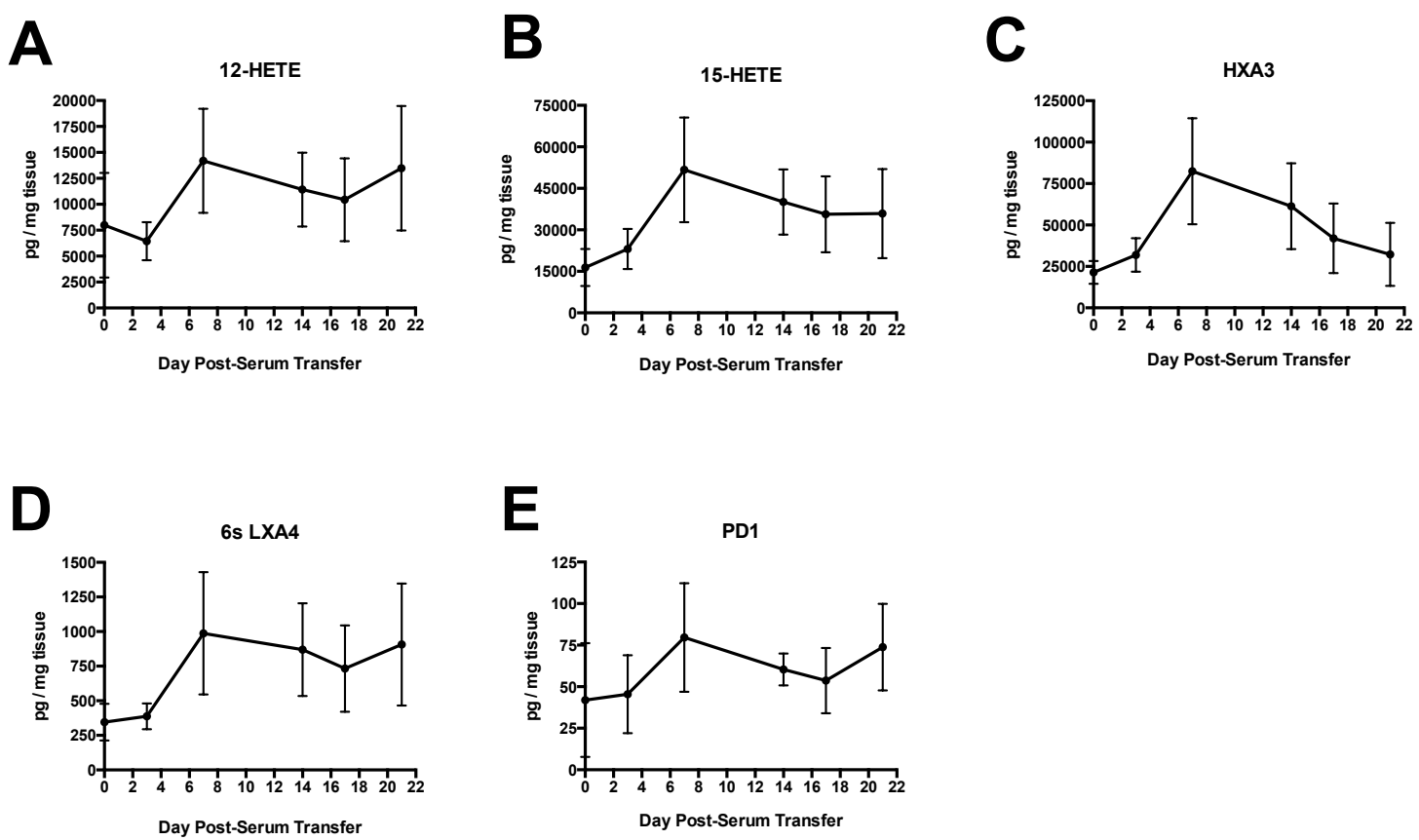

Fig. 6.9. 12/15-lipoxygenase (12/15-LOX)-derived metabolite production in the joints of arthritic mice. 12/15-lipoxygenase (12/15-LOX)-derived metabolites were quantified in the joints of mice that had received either $\mathrm{K} / \mathrm{BxN}$ or normal mouse serum using LC-MS/MS on days $0,3,7,14,17$, and 21 posttransfer. Values are means $\pm S D, n=5$ on day 0,9 on day 3 and 14,10 on day 7 and 21, and 8 on day 17 post-serum transfer. (Values by Dr. Darren S. Dumlao, figures constructed by Anna M. Cunningham.) 
A

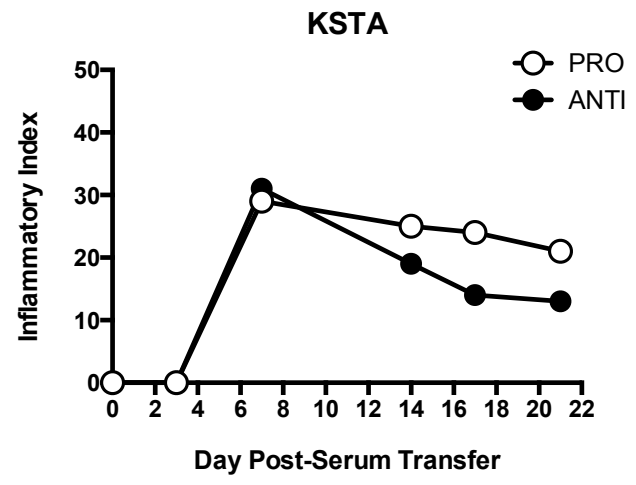

C

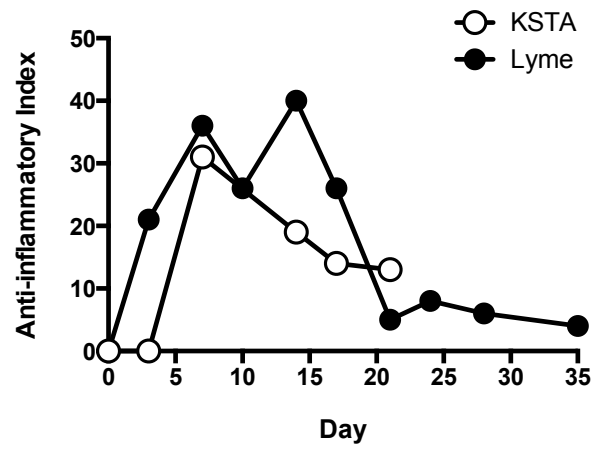

B

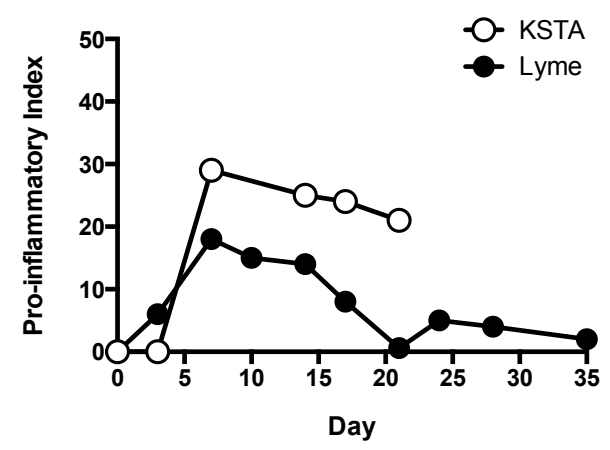


Fig. 6.10. Inflammatory indices of mice induced to have either KSTA or Lyme arthritis. Inflammatory indices were generated as described (300) for both the KSTA and Lyme arthritis models. Quantities of pro- or anti-inflammatory metabolites upregulated by a fold change of two or more were calculated in the KSTA model on days $0,3,7,14,17$, and 21 post-serum transfer and in the Lyme arthritis model on days $0,3,7,10,14,17,21,24,28$ and 35 post-infection. $n=5$ on day 0,9 on day 3 and 14,10 on day 7 and 21 , and 8 on day 17 post-serum transfer in the KSTA model, and $n=5$ for every time point in the Lyme arthritis model. Data represents the percentage of metabolites upregulated at each time point. (A) pro- and anti-inflammatory metabolite generation throughout the KSTA model, (B) pro-inflammatory metabolite production in the KSTA and Lyme arthritis models, and (C) anti-inflammatory metabolite production in the KSTA and Lyme arthritis models. (Eicosanoids were detected by Dr. Darren S. Dumlao using LC-MS/MS, inflammatory indices and figures were calculated and generated by Anna M. Cunningham.) 
suggests that the percentage of pro-inflammatory and anti-inflammatory metabolites were equivalent in control mice and on days 3 and 7 post-serum transfer; however, on days 14,17 , and 21 post-serum transfer, the percentage of pro-inflammatory metabolites was larger than the percentage of antiinflammatory metabolites. This data suggested that early during disease, antiinflammatory metabolites were produced in an effort to limit inflammation induced by potent inflammatory metabolites. This data may also suggest that the production of anti-inflammatory metabolites early during disease was important for subsequent resolution $(7,299)$. However, the predominance of proinflammatory metabolites at times during which inflammation was decreasing (fig. 6.1) is perplexing. A possible explanation for this observation is that the function of many of these metabolites varies depending on microenvironment and cellular composition. For example, $\mathrm{PGE}_{2}$ has been implicated to be proinflammatory when produced in low quantities and anti-inflammatory when produced in high quantities $(60,301)$. The function of $\mathrm{PGE}_{2}$ may also vary depending on cellular composition of the joint, as four receptors for $\mathrm{PGE}_{2}$ exist (EP1 - EP4) and are differentially expressed on distinct cell types (302). As the predominance of cells in joint tissue shifts from neutrophils during the peak of arthritis to macrophages during resolution (fig. 6.1 D), an alteration in eicosanoid receptor expression in the joint could affect the function of these eicosanoids.

To compare the production of lipid mediators in this model of arthritis to those produced in another well-established model, we calculated inflammatory indices from the lipidomic data that we accumulated using the Lyme arthritis 
model (144) (fig. 6.10 B, C). We found that the production of pro-inflammatory mediators was much higher in KSTA when compared to Lyme arthritis (fig. 6.10 B) and that the production of anti-inflammatory mediators was higher in Lyme arthritis (fig. 6.10 C). Inflammation resulting from Lyme arthritis does not reach the severity that is seen in KSTA, which may explain why pro-inflammatory indices for KSTA are much higher than those found in Lyme arthritis (fig. 6.10 B). Furthermore, Lyme arthritis is a transient arthritis that requires lipid mediators for the resolution of arthritis $(141,142)$, while resolution of KSTA correlates with the degradation of passaged anti-GPI autoantibodies. Thus, the higher antiinflammatory index found in Lyme arthritis may be explained by the greater role that these mediators play in the cessation of inflammation in this model (fig. $\mathbf{6 . 1 0}$ C).

\section{DISCUSSION}

The K/BxN serum transfer model of arthritis (KSTA) has proven to be a beneficial model for the study of human rheumatoid arthritis due to many shared symptoms and pathways mediating disease $(252,253)$. Eicosanoids are known to interact with immune pathways that mediate $\operatorname{KSTA}(109,110,259,263)$. Previous data has implicated a role for the enzymatic pathways 5-lipoxygenase (5-LOX) and cyclooxygenase-1 (COX-1) and their respective products, leukotriene B4 (LTB4) and prostaglandin $\mathrm{I}_{2}\left(\mathrm{PGI}_{2}\right)$, in the generation and maintenance of arthritis resulting from the transfer of anti-glucose-6-phosphate isomerase (GPI) autoantibody-containing $\mathrm{K} / \mathrm{BxN}$ serum $(109,259)$. 
To better understand which eicosanoid pathways were engaged during KSTA, we utilized a methodology we developed that uses liquid chromatographytandem mass spectrometry to temporally identify eicosanoids directly from arthritic tissues (144).

Using this methodology, we identified that the peak of eicosanoid generation during KSTA is day 7 (fig 6.3 B), correlating with the peak of arthritis severity (fig. 6.1). We found that the most commonly utilized eicosanoid enzymatic pathway was the cytochrome P450 (CYP) pathway (fig. 6.3 A). This pathway produces the vasoactive HETEs and EETs. Vasodilatory EETs were expressed to higher levels than the vasoconstrictive HETEs (fig. 6.4, 6.5), suggesting that the overall vascular tone during the peak of arthritis was conducive to vascular leakage and the infiltration of immune cells, thus promoting inflammation.

Non-enzymatically-produced metabolites were also dynamically regulated throughout the course of KSTA (fig. 6.3 A, fig. 6.6), suggesting that the joints of arthritic mice consist of a highly oxidative microenvironment that likely contribute to the severity of inflammation. This is consistent with studies indicating that molecules that limit restrain oxidative stress, such as heme-oxygenase $1(\mathrm{HO}-1)$, are important for limiting severity of KSTA disease (283).

The production of $\mathrm{LTB}_{4}$ was elevated throughout the course of KSTA (fig. 6.8 A), which is consistent with data indicating that this molecule mediates the recruitment of neutrophils and stimulation of fibroblast-like synoviocytes (FLS) at numerous stages of this disease. The production of $\mathrm{PGF}_{1 \alpha}$, the breakdown 
product of $\mathrm{PGI}_{2}$, was elevated on days 7 and 21 post-serum transfer (fig. 6.7 A). As $\mathrm{PGI}_{2}$ is known to regulate the development of disease in this model, the production of $\mathrm{PGF}_{1 \alpha}$ at the peak of disease, day 7, makes sense. However, the reason for its production at day 21 , a time point at which arthritis has been resolved, is less clear. Studies have indicated that the production of $\mathrm{PGI}_{2}$ is stimulated by IL-6 produced subsequent to IL-1 $\beta$ stimulation of synovial fibroblasts (290). The development of KSTA is mediated by a cascade of neutrophil recruitment, mediated in part by $\mathrm{LTB}_{4}$ and IL-1 $\beta$, resulting in the proliferation of invasive and destructive FLS $(110,261)$. C5a stimulation of the "first wave" of $\mathrm{LTB}_{4}$-producing neutrophils leads to the recruitment of immune complex-stimulated, IL-1 $\beta$-producing neutrophils (261). Although the production of $\mathrm{PGF}_{1 \alpha}$ was detected at day 21 post-serum transfer, this molecule is a breakdown product of $\mathrm{PGI}_{2}$ and thus could indicate that $P \mathrm{PI}_{2}$ was being produced at slightly earlier time points. Furthermore, the occurrence of this molecule late in disease may corroborate data that suggests that neutrophils produced later in disease (the "second" wave of IL-1 $\beta$-producing neutrophils) are important for the induction of disease-modulating $\mathrm{PGI}_{2}$.

Although previous data suggests that COX-2 does not play a role in modulating KSTA (259), we detected high levels of metabolites produced via this pathway (fig. 6.7 D, E). We have previously shown that COX-2 mediates the resolution of Lyme arthritis (141). It is possible that anti-inflammatory COX-2 metabolites are produced at the peak of KSTA in an effort to induce resolution of arthritis. However, due to the fact that the resolution of KSTA is mediated by the 
degradation of passaged autoantibodies, it is unlikely that any effects would be seen in the absence of COX-2. Thus, the role of COX-2-derived metabolites in the regulation of KSTA remains somewhat unclear.

We have previously conducted a similar study utilizing the same lipidomics methodology to detect the production of eicosanoids throughout the course of Lyme arthritis (144). In the Lyme arthritis study, similar to the present study, we found that the majority of eicosanoids were produced at the peak of disease, day 10 post-infection. Interestingly, all eicosanoids were produced at much higher levels in joints of mice induced to have KSTA when compared to mice with Lyme arthritis. In an effort to better understand this discovery, we generated pro- and anti-inflammatory scores by calculating the percentage of metabolites that were affected by a fold change of 2 or more with known pro- or anti-inflammatory functions (300) (fig. 6.10). Pro-inflammatory metabolites were produced at much higher levels in the joints of mice with KSTA while the joints of mice with Lyme arthritis tended to produce more anti-inflammatory metabolites (fig. $6.10 \mathrm{~B}, \mathrm{C}$ ). This data emphasizes the fact that inflammation resulting from KSTA is much more severe than inflammation resulting from Lyme arthritis (compare severity reported in Blaho, et al. 2009 (144) and fig. 6.1), but also highlights the fact that pro-resolving metabolites are necessary for resolution of Lyme arthritis but dispensable for the resolution of KSTA. The increased severity in this model is likely responsible for the increased expression of eicosanoids measured in this study. 
The data presented herein is consistent with studies indicating that COX1-derived $\mathrm{PGI}_{2}$ and 5-LOX-derived $\mathrm{LTB}_{4}$ are involved in disease associated with KSTA. Furthermore, our data suggests that pathways that regulate vascular tone (CYP) and oxidative stress (non-enzymatic) are heavily involved in the development of this disease. The methodology used to obtain these results is a useful tool for the identification of pathways and metabolites that regulate inflammation resulting from various forms of disease. These results emphasize the fact that eicosanoids are dynamically regulated throughout the course of arthritis and are important mediators of inflammation. 


\section{CHAPTER SEVEN}

\section{CONCLUSIONS}

Lyme disease was first described by Steere, et al. in 1977 after an outbreak of an apparent juvenile rheumatic disease in Lyme, Connecticut (122, 303-306). Epidemiological data suggested transmission of the disease occurred via tick vector, specifically via the Ixodes species of ticks (the black-legged deer tick, Ixodes scapularis) (307). It was not until1982 that the causative spirochete was isolated from an Ixodes tick and named for its discoverer, Dr. Willy Burgdorfer: Borrelia burgdorferi $(B b)(123)$.

Early clinical manifestations of human Lyme disease include mild flu-like symptoms and a bull's eye-shaped rash termed erythema migrans (EM). Later stage symptoms include arthritis of the large joints and carditis (124). In 1990, CD-1 mice were identified as a beneficial animal model with which to study Lyme disease as inoculation with $B b$ resulted in disease that was comparable to that observed in humans: severe arthritis and carditis (308).

It was observed early on that a genetic component determining susceptibility to murine Lyme disease existed. In 1990, Barthold, et al. surveyed five strains of mice and determined that while all mice were susceptible to infection with $B b$, only two out of the five strains developed severe arthritis following inoculation with $B b[\mathrm{C} 3 \mathrm{H} / \mathrm{He}(\mathrm{C} 3 \mathrm{H})$ and $\mathrm{SWR}]$ and the other three strains remained resistant to disease [C57BL/6 (B6), BALB/c, and SJL] (130). Using $\mathrm{C} 3 \mathrm{H}$ and $\mathrm{B} 6$ mice with or without the beige (bg) mutation, which impairs 
granulocyte and NK cell function, Barthold et al. determined that genetic susceptibility to murine Lyme arthritis was likely linked to granulocyte function (309).

In 2003, Brown et al. discovered that the chemokines KC and MCP-1 were upregulated in genetically susceptible $\mathrm{C} 3 \mathrm{H}$ mice compared to resistant $\mathrm{B} 6$ mice (132). While $\mathrm{C} 3 \mathrm{H}$ mice deficient in CCR2, the receptor for MCP-1, remained susceptible to disease following inoculation with $\mathrm{Bb}, \mathrm{C} 3 \mathrm{H}$ mice deficient in the chemokine receptor CXCR2 were resistant to disease. In the study described in chapter three of this thesis, $\mathrm{C} 3 \mathrm{H} \mathrm{KC}^{-/-}$mice were used to determine definitively that the chemokine $\mathrm{KC}$ signaling through $\mathrm{CXCR} 2$ is responsible for neutrophil recruitment and subsequent development of both arthritis and carditis following inoculation with $B b$. Furthermore, the CXCR2 ligands MIP-2 and LIX were unable to compensate for the loss of KC signaling through CXCR2. These studies were the first to determine an immunological regulatory mechanism mediating disease susceptibility to murine Lyme disease. Future studies would do well to determine potential genetic mechanisms that regulate $\mathrm{KC}$ transcription and translation, as these may aid in the determination of genetic factors that mediate murine susceptibility or resistance to Lyme disease. As p38 mitogen activated protein kinase (p38 MAPK) is known to mediate the production of proinflammatory cytokines in response to $B b$ (likely including KC) (140), further investigation of differences in this pathway may aid in the clarification of why B6 mice produce so much less $\mathrm{KC}$ compared to $\mathrm{C} 3 \mathrm{H}$ mice and thus further elucidate 
mechanisms responsible for determining genetic susceptibility or resistance to murine Lyme disease.

Although genetic factors regulate the susceptibility or resistance of mice to Lyme disease, many other factors are known to mediate the severity of disease in susceptible mice. One such group of factors is the small, immuno-regulatory eicosanoids (310). In 2009, Blaho et al. isolated lipids directly from the ankle joints of $B b$-infected mice and used lipidomics to show dynamic expression of eicosanoids throughout the course of disease (144). Pro-inflammatory prostaglandins and leukotrienes were produced in the early stages of Lyme arthritis and pro-resolving molecules (such as protectin D1) were produced at the peak of arthritis severity, likely in an effort to promote resolution.

Studies using mice genetically deficient in eicosanoid biosynthetic enzymes such as $5-\mathrm{LOX}^{-{ }^{-}}, \mathrm{COX}-1^{--_{-}}$, and $\mathrm{COX}-2^{-/-}$have determined that these pathways are important for the regulation of various aspects of the immune response to $B b . \quad 5-L O X$ is responsible for the production of pro-inflammatory leukotrienes, but is also required for the production of the anti-inflammatory/proresolving lipoxins, protectins, and resolvins. Mice deficient in 5-LOX were able to mount an effective immune response to $B b$ and developed arthritis normally, but were unable to resolve arthritic inflammation, suggesting that 5-LOX deficiency results in defective pro-resolution mechanisms (142). COX-1/- mice had no noticeable defect in the ability to develop or resolve arthritic inflammation in response to $B b$, but were unable to form functional germinal centers and produce IL-6 and IL-17 (143). 
Inhibition of the enzyme COX-2 resulted in attenuated arthritis severity and the development chronic arthritis, suggesting that metabolites produced via this pathway are responsible for both pro-inflammatory and pro-resolution mechanisms (141). Lipidomic analysis of $C O X-2^{-/-}$mice revealed that interference with this pathway also negatively impacts the production of lipoxygenase metabolites, indicating that defects in the pro- and antiinflammatory machinery resulting from COX-2 inhibition could be due to decreased prostaglandin, leukotriene, lipoxin, protectin, and/or resolvin production (144).

In chapter six of this thesis, lipid and cytokine analysis was performed in order to further elucidate the global effects of COX-2 inhibition. Interestingly, results from this study varied from those previously described by Blaho, et al. Blaho, et al. observed that mice treated with the COX-2 inhibitor Celecoxib developed persistent arthritis that was unable to resolve by day 35 post-infection (141). In the study presented herein, mice treated with Celecoxib had fully resolved arthritis by day 62 post-infection. This data suggests the possibility that COX-2 inhibition does not prevent resolution, but rather delays it significantly. As the study explained in chapter six involved feeding mice Celecoxib for two weeks prior to infection with $B b$ and Blaho et al. only fed mice Celecoxib starting on day -1 prior to $B b$ infection, it is also possible that such an extended time of COX-2 inhibition resulted in the development of compensatory mechanisms altering the outcome of the experiment. However, the latter explanation is unlikely, as 
studies exploring COX-2 inhibition to date have not identified any functional compensatory mechanisms.

Of the several metabolites produced via the COX-2 pathway, perhaps the best recognized is $\mathrm{PGE}_{2}$. In the context of inflammation, $\mathrm{PGE}_{2}$ has classically been considered pro-inflammatory due to its ability to stimulate tissue edema and cytokine production (206). However, recent studies have determined that $\mathrm{PGE}_{2}$ may actually function as a "switch" that mediates the shift from pro-inflammatory to pro-resolving metabolites (82). It is possible, then, that the absence of $\mathrm{PGE}_{2}$ signaling during COX-2 deficiency or inhibition is responsible for the lack of Lyme arthritis resolution. $\mathrm{PGE}_{2}$ has 4 difference receptors (EP1, 2, 3, and 4). EP2 and EP4 have been implicated the most for arthritic inflammation. In chapter six, we treated COX $-2^{-/-}$mice with Butaprost, an agonist of the EP2 receptor, and found that this treatment restored resolution in the $\mathrm{COX}-2^{-/-}$mice. This data suggested that defective resolution observed during COX-2 inhibition or deficiency results from a lack of $P G E_{2}$ signaling through the EP2 receptor. Future studies would benefit from further exploring the role $\mathrm{PGE}_{2}$ plays in Lyme arthritis and mechanisms by which it may induce pro-resolution mechanisms such as the production of lipoxin.

The majority of eicosanoids studied in the context of inflammation are derived from the $\omega-6$ fatty acids (2-series prostaglandins and 4-series leukotrienes derived from linoleic and arachidonic acid). Metabolism of $\omega-3$ fatty acids (EPA and DHA) results in the production of the 3-series prostaglandins and 5-series leukotrienes. $\omega$-3-derived eicosanoids are much less bioactive than 
their $\omega$-6-derived counterparts and recent data has suggested that dietary supplementation with $\omega-3$ fatty acids (via consumption of fish oil) may be useful for the alleviation of immune disorders. Studies have shown that $\omega-3$ supplementation may be beneficial for the mitigation of symptoms associated with diabetes, cystic fibrosis, cardiovascular disease, cancer, and rheumatoid arthritis $(81,210-214)$, although the mechanisms by which fish oil/ $\omega-3$ fatty acids mediate these anti-inflammatory effects are poorly understood.

In chapter four of this thesis, mice were fed a diet in which the fatty acid source was fish oil (compared to the control diet, in which the fatty acid source was soy oil) and eicosanoid production was assessed throughout the course of Lyme arthritis. A previous study using a murine model of influenza observed that dietary fish oil supplementation resulted in attenuated lung inflammation, but increased mortality due to significantly increased viral titers in the lung (311). Therefore, the predicted outcome of the study in chapter four was that arthritis severity would be reduced in mice fed the fish oil diet and that these mice would have higher $B b$ titers. As the enzymes responsible for eicosanoid production would be utilizing the supposedly more anti-inflammatory $\omega-3$ fatty acid as substrates, it was hypothesized that the production of eicosanoids would be skewed toward anti-inflammatory/pro-resolving metabolites.

Rather than a skewing of pro- to anti-inflammatory metabolites, a global shift in eicosanoid production was observed in the fish-oil fed mice where the vast majority of eicosanoids were derived from $\omega-3$ fatty acids. Although this would perhaps lead one to suspect that disease would be attenuated in these 
mice, this was not the case. Fish oil-fed mice and control diet-fed mice had similar disease and $B b$ titers. This suggested that although fish oil may lead to attenuated inflammation in some models, it is not a sufficient anti-inflammatory for Lyme arthritis. It is important to note, however, that the quantity of fish oil that was included in the experimental diet far exceeds what is possible for human dietary supplementation. Therefore, it is possible that a threshold exists determining the therapeutically effectiveness of fish oil and that threshold was exceeded in this experiment.

Interestingly, although the vast majority of eicosanoids produced in the ankle joints of fish oil-fed mice were of $\omega-3$ origin, one $\omega$-6-derived eicosanoid was increased compared to control diet-fed mice: the $\mathrm{PGD}_{2}$ dehydration product $15 \mathrm{~d}-\mathrm{PG} \mathrm{J}_{2}$. As previously mentioned, this metabolite is considered antiinflammatory due to its inhibitory effects on NF-KB. Expression of this metabolite in the fish oil-fed mice reached levels at which $\mathrm{PGE}_{2}$ was produced in the control diet-fed mice. Upon further analysis, it was determined that fish oil-fed mice accumulated higher levels of $\mathrm{PGD}_{2}$-related products than $\mathrm{PGE}_{2}$-related products. This data suggests that fish oil could potentially exert anti-inflammatory activity via altered prostaglandin synthase activity. While soy oil results in the preferential production of PGE synthase products, fish oil results in the preferential production of PGD synthase products. Further investigation of the effects of $\omega-3$ fatty acids on prostaglandin synthase usage could potentially identify novel anti-inflammatory mechanisms of dietary fish oil supplementation. 
Human arthritis is a grouping of over 100 disorders characterized by joint inflammation, pain, stiffness, and swelling. As the instigating source of joint inflammation is typically unknown, treatments for most arthritides focus on managing pain and reducing symptoms to allow patients to carry on with normal life. Non-steroidal anti-inflammatory drugs (NSAIDs) have been extensively used in the management of pain and inflammation associated with arthritis. NSAIDs function by inhibiting pathways responsible for eicosanoid generation.

As described in chapter six, it is well established that eicosanoids play a role in the pathogenesis of Lyme arthritis and inhibition of eicosanoid biosynthetic pathways results in altered disease states upon infection with $B b$. However, inhibition of these pathways in other models of arthritis does not result in the same effects. In chapter five, the production of eicosanoids during a different model of arthritis, K/BxN serum transfer arthritis (KSTA), was assessed. This model of arthritis is autoimmune in nature. Its development is reliant on the passage of autoantibodies and its cessation correlates with the natural degradation of these autoantibodies. While deficiency of COX-1 during Lyme arthritis has no discernable effect on arthritis development or resolution, COX-1 deficiency during KSTA results in attenuated disease (259). Furthermore, while 5-LOX deficiency during Lyme arthritis has not effect on the development of arthritis, resolution in these mice is defective. However, development of KSTA is dependent on the 5-LOX metabolite $\mathrm{LTB}_{4}$ signaling through its cognate receptors BLT-1 and BLT-2 (109). 
Global eicosanoid production in the ankle joints of mice induced to have KSTA was compared to ankle joint eicosanoid production during Lyme arthritis. Pro-inflammatory mediators were produced in greater quantities during KSTA compared to Lyme arthritis, which most likely represented the fact that joint inflammation is much more severe in the model of KSTA. It was also observed that anti-inflammatory metabolites were produced in higher quantities during Lyme arthritis than KSTA. As Lyme arthritis requires active resolution while resolution of KSTA is associated with the passive degradation of transferred autoantibodies, the higher quantities of anti-inflammatory metabolites produced in Lyme arthritis may indicate the greater need for them for the induction of resolution in this model.

The differences observed in the model of Lyme arthritis and KSTA highlight an important caveat to arthritis research: not all arthritides are the same. As mentioned previously, arthritis is actually the collection of 100 different disorders with similar symptoms. In order to translate laboratory findings to human medicine and potential therapeutics for various disorders, it is vital to use numerous animal models.

The studies contained in this thesis contribute greatly to the understanding of Lyme arthritis pathogenesis. From the elucidation of chemokine pathways responsible for the development of Lyme arthritis to the appreciation of the many ways which eicosanoids regulate disease in this model, the work presented herein serves as a strong foundation for future studies to be built upon. Further understanding of the pathogenesis of murine Lyme arthritis will aid in the 
development of more sophisticated therapeutics that can be used for the treatment of human Lyme arthritis and other forms of arthritis, as well. 


\section{REFERENCES}

1. Majno G. The Healing Hand: Man and Wound in the Ancient World: Harvard University Press; 1975.

2. Majno G, Johns I. Cells, Tissues and Disease: Oxford University Press; 2004.

3. Barton GM. A calculated response: control of inflammation by the innate immune system. J Clin Invest. 2008;118(2):413-20. PMCID: 2214713.

4. Pober JS, Sessa WC. Evolving functions of endothelial cells in inflammation. Nat Rev Immunol. 2007;7(10):803-15.

5. Nathan C. Neutrophils and immunity: challenges and opportunities. Nat Rev Immunol. 2006;6(3):173-82.

6. Nathan C. Points of control in inflammation. Nature. 2002;420(6917):84652.

7. Serhan CN, Savill J. Resolution of inflammation: the beginning programs the end. Nat Immunol. 2005;6(12):1191-7.

8. Medzhitov R. Origin and physiological roles of inflammation. Nature. 2008;454(7203):428-35.

9. Serhan CN. Lipoxins and aspirin-triggered 15-epi-lipoxins are the first lipid mediators of endogenous anti-inflammation and resolution. Prostaglandins Leukot Essent Fatty Acids. 2005;73(3-4):141-62.

10. Serhan CN. Novel eicosanoid and docosanoid mediators: resolvins, docosatrienes, and neuroprotectins. Curr Opin Clin Nutr Metab Care. 2005;8(2):115-21.

11. Serhan CN. Novel omega -- 3-derived local mediators in anti-inflammation and resolution. Pharmacol Ther. 2005;105(1):7-21.

12. Kalinski P. Regulation of immune responses by prostaglandin e2. J Immunol. 2012;188(1):21-8.

13. Gilroy DW, Colville-Nash PR. New insights into the role of COX 2 in inflammation. J Mol Med. 2000;78(3):121-9. 
14. Curtis-Prior P. The Eicosanoids: John Wiley and Sons, Ltd.; 2004.

15. Anggard E, Samuelsson B. Biosynthesis of prostaglandins from arachidonic acid in guinea pig lung. Prostaglandins and related factors. 38. J Biol Chem. 1965;240(9):3518-21.

16. Simopoulos AP. Omega-3 fatty acids in health and disease and in growth and development. Am J Clin Nutr. 1991;54(3):438-63.

17. James MJ, Gibson RA, Cleland LG. Dietary polyunsaturated fatty acids and inflammatory mediator production. Am J Clin Nutr. 2000;71(1 Suppl):343S$8 \mathrm{~S}$.

18. Taber L, Chiu CH, Whelan J. Assessment of the arachidonic acid content in foods commonly consumed in the American diet. Lipids. 1998;33(12):1151-7.

19. Goodsell DS. The molecular perspective: cyclooxygenase-2. Oncologist. 2000;5(2):169-71.

20. Smith WL, DeWitt DL, Garavito RM. Cyclooxygenases: structural, cellular, and molecular biology. Annu Rev Biochem. 2000;69:145-82.

21. Panigrahy D, Kaipainen A, Greene ER, Huang S. Cytochrome P450derived eicosanoids: the neglected pathway in cancer. Cancer Metastasis Rev. 2010;29(4):723-35. PMCID: 2962793.

22. Fries S, Grosser T. The cardiovascular pharmacology of COX-2 inhibition. Hematology Am Soc Hematol Educ Program. 2005:445-51.

23. Ackerman WEt, Robinson JM, Kniss DA. Despite transcriptional and functional coordination, cyclooxygenase-2 and microsomal prostaglandin $\mathrm{E}$ synthase-1 largely reside in distinct lipid microdomains in WISH epithelial cells. J Histochem Cytochem. 2005;53(11):1391-401. PMCID: 1282464.

24. Morita I, Schindler M, Regier MK, Otto JC, Hori T, DeWitt DL, et al. Different intracellular locations for prostaglandin endoperoxide $\mathrm{H}$ synthase-1 and -2. J Biol Chem. 1995;270(18):10902-8.

25. Williams JA, Shacter E. Regulation of macrophage cytokine production by prostaglandin E2. Distinct roles of cyclooxygenase-1 and -2. J Biol Chem. 1997;272(41):25693-9. 
26. Masferrer JL, Seibert K, Zweifel B, Needleman P. Endogenous glucocorticoids regulate an inducible cyclooxygenase enzyme. Proc Natl Acad Sci U S A. 1992;89(9):3917-21. PMCID: 525602.

27. Mitchell JA, Evans TW. Cyclooxygenase-2 as a therapeutic target. Inflamm Res. 1998;47 Suppl 2:S88-92.

28. Smith CJ, Zhang Y, Koboldt CM, Muhammad J, Zweifel BS, Shaffer A, et al. Pharmacological analysis of cyclooxygenase-1 in inflammation. Proc Natl Acad Sci U S A. 1998;95(22):13313-8. PMCID: 23795.

29. Demasi M, Caughey GE, James MJ, Cleland LG. Assay of cyclooxygenase-1 and 2 in human monocytes. Inflamm Res. 2000;49(12):73743.

30. Hamberg $M$, Hamberg $G$. On the mechanism of the oxygenation of arachidonic acid by human platelet lipoxygenase. Biochem Biophys Res Commun. 1980;95(3):1090-7.

31. Nugteren DH. Arachidonic acid-12-lipoxygenase from bovine platelets. Methods Enzymol. 1982;86:49-54.

32. Narumiya S, Salmon JA, Cottee FH, Weatherley BC, Flower RJ. Arachidonic acid 15-lipoxygenase from rabbit peritoneal polymorphonuclear leukocytes. Partial purification and properties. J Biol Chem. 1981;256(18):958392.

33. Peters-Golden M, Brock TG. 5-lipoxygenase and FLAP. Prostaglandins Leukot Essent Fatty Acids. 2003;69(2-3):99-109.

34. Conrad DJ. The arachidonate 12/15 lipoxygenases. A review of tissue expression and biologic function. Clin Rev Allergy Immunol. 1999;17(1-2):71-89.

35. Reilly MP, Lawson JA, FitzGerald GA. Eicosanoids and isoeicosanoids: indices of cellular function and oxidant stress. J Nutr. 1998;128(2 Suppl):434S$8 \mathrm{~S}$.

36. Steinhilber D, Fischer AS, Metzner J, Steinbrink SD, Roos J, Ruthardt M, et al. 5-lipoxygenase: underappreciated role of a pro-inflammatory enzyme in tumorigenesis. Front Pharmacol. 2010;1:143. PMCID: 3153017. 
37. Panossian A, Hamberg M, Samuelsson B. On the mechanism of biosynthesis of leukotrienes and related compounds. FEBS Lett. 1982;150(2):511-3.

38. Borgeat P, Samuelsson B. Arachidonic acid metabolism in polymorphonuclear leukocytes: unstable intermediate in formation of dihydroxy acids. Proc Natl Acad Sci U S A. 1979;76(7):3213-7. PMCID: 383794.

39. Borgeat P, Samuelsson B. Arachidonic acid metabolism in polymorphonuclear leukocytes: effects of ionophore A23187. Proc Natl Acad Sci U S A. 1979;76(5):2148-52. PMCID: 383554.

40. Radmark O, Malmsten C, Samuelsson B, Clark DA, Goto G, Marfat A, et al. Leukotriene A: stereochemistry and enzymatic conversion to leukotriene B. Biochem Biophys Res Commun. 1980;92(3):954-61.

41. Radmark O, Malmsten C, Samuelsson B. Leukotriene A4: enzymatic conversion to leukotriene C4. Biochem Biophys Res Commun. 1980;96(4):167987.

42. Orning L, Hammarstrom S, Samuelsson B. Leukotriene D: a slow reacting substance from rat basophilic leukemia cells. Proc Natl Acad Sci U S A. 1980;77(4):2014-7. PMCID: 348641.

43. Sok DE, Pai JK, Atrache V, Kang YC, Sih CJ. Enzymatic inactivation of SRS-Cys-Gly (leukotriene D). Biochem Biophys Res Commun. 1981;101(1):2229.

44. Edenius $\mathrm{C}$, Heidvall $\mathrm{K}$, Lindgren JA. Novel transcellular interaction: conversion of granulocyte-derived leukotriene A4 to cysteinyl-containing leukotrienes by human platelets. Eur J Biochem. 1988;178(1):81-6.

45. Samuelsson B, Dahlen SE, Lindgren JA, Rouzer CA, Serhan CN. Leukotrienes and lipoxins: structures, biosynthesis, and biological effects. Science. 1987;237(4819):1171-6.

46. Halter F, Tarnawski AS, Schmassmann A, Peskar BM. Cyclooxygenase 2implications on maintenance of gastric mucosal integrity and ulcer healing: controversial issues and perspectives. Gut. 2001;49(3):443-53. PMCID: 1728453. 
47. Lipsky PE, Brooks P, Crofford LJ, DuBois R, Graham D, Simon LS, et al. Unresolved issues in the role of cyclooxygenase-2 in normal physiologic processes and disease. Arch Intern Med. 2000;160(7):913-20.

48. Yu Y, Stubbe J, Ibrahim S, Song WL, Smyth EM, Funk CD, et al. Cyclooxygenase-2-dependent prostacyclin formation and blood pressure homeostasis: targeted exchange of cyclooxygenase isoforms in mice. Circ Res. 2010;106(2):337-45. PMCID: 2818801.

49. Grosser T, Fries S, FitzGerald GA. Biological basis for the cardiovascular consequences of COX-2 inhibition: therapeutic challenges and opportunities. J Clin Invest. 2006;116(1):4-15. PMCID: 1323269.

50. Marnett LJ. The COXIB experience: a look in the rearview mirror. Annu Rev Pharmacol Toxicol. 2009;49:265-90.

51. Langenbach R, Loftin C, Lee C, Tiano H. Cyclooxygenase knockout mice: models for elucidating isoform-specific functions. Biochem Pharmacol. 1999;58(8):1237-46.

52. Masferrer JL, Isakson PC, Seibert K. Cyclooxygenase-2 inhibitors: a new class of anti-inflammatory agents that spare the gastrointestinal tract. Gastroenterol Clin North Am. 1996;25(2):363-72.

53. Simmons DL, Botting RM, Hla T. Cyclooxygenase isozymes: the biology of prostaglandin synthesis and inhibition. Pharmacol Rev. 2004;56(3):387-437.

54. Meyer F, Ramanujam KS, Gobert AP, James SP, Wilson KT. Cutting edge: cyclooxygenase-2 activation suppresses Th1 polarization in response to Helicobacter pylori. J Immunol. 2003;171(8):3913-7.

55. Przybylkowski A, Kurkowska-Jastrzebska I, Joniec I, Ciesielska A, Czlonkowska A, Czlonkowski A. Cyclooxygenases mRNA and protein expression in striata in the experimental mouse model of Parkinson's disease induced by 1methyl-4-phenyl-1,2,3,6-tetrahydropyridine administration to mouse. Brain Res. 2004;1019(1-2):144-51.

56. Bazan NG. COX-2 as a multifunctional neuronal modulator. Nat Med. 2001;7(4):414-5.

57. Gray PA, Warner TD, Vojnovic I, Del Soldato P, Parikh A, Scadding GK, et al. Effects of non-steroidal anti-inflammatory drugs on cyclo-oxygenase and 
lipoxygenase activity in whole blood from aspirin-sensitive asthmatics vs healthy donors. Br J Pharmacol. 2002;137(7):1031-8. PMCID: 1573571.

58. Hu KQ. Cyclooxygenase 2 (COX2)-prostanoid pathway and liver diseases. Prostaglandins Leukot Essent Fatty Acids. 2003;69(5):329-37.

59. Mardini IA, FitzGerald GA. Selective inhibitors of cyclooxygenase-2: a growing class of anti-inflammatory drugs. Mol Interv. 2001;1(1):30-8.

60. Gilroy DW, Colville-Nash PR, Willis D, Chivers J, Paul-Clark MJ, Willoughby DA. Inducible cyclooxygenase may have anti-inflammatory properties. Nat Med. 1999;5(6):698-701.

61. Zhao J, Legge K, Perlman S. Age-related increases in PGD(2) expression impair respiratory DC migration, resulting in diminished $T$ cell responses upon respiratory virus infection in mice. J Clin Invest. 2011;121(12):4921-30. PMCID: 3226008.

62. Arima $M$, Fukuda $T$. Prostaglandin $D(2)$ and $T(H) 2$ inflammation in the pathogenesis of bronchial asthma. Korean J Intern Med. 2011;26(1):8-18. PMCID: 3056260.

63. Ahmed SR, McGettrick HM, Yates CM, Buckley CD, Ratcliffe MJ, Nash GB, et al. Prostaglandin D2 Regulates CD4+ Memory T Cell Trafficking across Blood Vascular Endothelium and Primes These Cells for Clearance across Lymphatic Endothelium. J Immunol. 2011.

64. Yamamoto Y, Otani S, Hirai H, Nagata K, Aritake K, Urade Y, et al. Dual Functions of Prostaglandin D2 in Murine Contact Hypersensitivity via DP and CRTH2. Am J Pathol. 2011;179(1):302-14. PMCID: 3123801.

65. Powell WS. 15-Deoxy-delta12,14-PGJ2: endogenous PPARgamma ligand or minor eicosanoid degradation product? J Clin Invest. 2003;112(6):828-30. PMCID: 193674.

66. Scher JU, Pillinger MH. 15d-PGJ2: the anti-inflammatory prostaglandin? Clin Immunol. 2005;114(2):100-9.

67. Bell-Parikh LC, Ide T, Lawson JA, McNamara P, Reilly M, FitzGerald GA. Biosynthesis of 15-deoxy-delta12,14-PGJ2 and the ligation of PPARgamma. J Clin Invest. 2003;112(6):945-55. PMCID: 193665. 
68. Gilroy DW, Colville-Nash PR, McMaster S, Sawatzky DA, Willoughby DA, Lawrence T. Inducible cyclooxygenase-derived 15-deoxy(Delta)12-14PGJ2 brings about acute inflammatory resolution in rat pleurisy by inducing neutrophil and macrophage apoptosis. FASEB J. 2003;17(15):2269-71.

69. Abrahao AC, Castilho RM, Squarize $\mathrm{CH}$, Molinolo AA, dos Santos-Pinto D, Jr., Gutkind JS. A role for COX2-derived PGE2 and PGE2-receptor subtypes in head and neck squamous carcinoma cell proliferation. Oral Oncol. 2010;46(12):880-7. PMCID: 2991505.

70. Bankhurst $A D$. The modulation of human natural killer cell activity by prostaglandins. J Clin Lab Immunol. 1982;7(2):85-91.

71. Akasaki Y, Liu G, Chung NH, Ehtesham M, Black KL, Yu JS. Induction of a CD4+ $T$ regulatory type 1 response by cyclooxygenase-2-overexpressing glioma. J Immunol. 2004;173(7):4352-9.

72. Smith RJ. Modulation of phagocytosis by and lysosomal enzyme secretion from guinea-pig neutrophils: effect of nonsteroid anti-inflammatory agents and prostaglindins. J Pharmacol Exp Ther. 1977;200(3):647-57.

73. Aronoff DM, Canetti C, Peters-Golden M. Prostaglandin E2 inhibits alveolar macrophage phagocytosis through an E-prostanoid 2 receptor-mediated increase in intracellular cyclic AMP. J Immunol. 2004;173(1):559-65.

74. Babaev VR, Chew JD, Ding L, Davis S, Breyer MD, Breyer RM, et al. Macrophage EP4 deficiency increases apoptosis and suppresses early atherosclerosis. Cell Metab. 2008;8(6):492-501. PMCID: 2614698.

75. Becker MR, Siegelin MD, Rompel R, Enk AH, Gaiser T. COX-2 expression in malignant melanoma: a novel prognostic marker? Melanoma Res. 2009;19(1):8-16.

76. Algra AM, Rothwell PM. Effects of regular aspirin on long-term cancer incidence and metastasis: a systematic comparison of evidence from observational studies versus randomised trials. Lancet Oncol. 2012.

77. Rothwell PM, Fowkes FG, Belch JF, Ogawa H, Warlow CP, Meade TW. Effect of daily aspirin on long-term risk of death due to cancer: analysis of individual patient data from randomised trials. Lancet. 2011;377(9759):31-41. 
78. Rothwell PM, Price JF, Fowkes FG, Zanchetti A, Roncaglioni MC, Tognoni $G$, et al. Short-term effects of daily aspirin on cancer incidence, mortality, and non-vascular death: analysis of the time course of risks and benefits in 51 randomised controlled trials. Lancet. 2012.

79. Rothwell PM, Wilson M, Elwin CE, Norrving B, Algra A, Warlow CP, et al. Long-term effect of aspirin on colorectal cancer incidence and mortality: 20-year follow-up of five randomised trials. Lancet. 2010;376(9754):1741-50.

80. Rothwell PM, Wilson M, Price JF, Belch JF, Meade TW, Mehta Z. Effect of daily aspirin on risk of cancer metastasis: a study of incident cancers during randomised controlled trials. Lancet. 2012.

81. Yokoyama $M$, Origasa $H$, Matsuzaki $M$, Matsuzawa $Y$, Saito $Y$, Ishikawa $Y$, et al. Effects of eicosapentaenoic acid on major coronary events in hypercholesterolaemic patients (JELIS): a randomised open-label, blinded endpoint analysis. Lancet. 2007;369(9567):1090-8.

82. Levy BD, Clish CB, Schmidt B, Gronert K, Serhan CN. Lipid mediator class switching during acute inflammation: signals in resolution. Nat Immunol. 2001;2(7):612-9.

83. Chan MM, Moore AR. Resolution of inflammation in murine autoimmune arthritis is disrupted by cyclooxygenase-2 inhibition and restored by prostaglandin E2-mediated lipoxin A4 production. J Immunol. 2010;184(11):6418-26. PMCID: 3032587.

84. Grainger JR, Wohlfert EA, Fuss IJ, Bouladoux N, Askenase MH, Legrand $\mathrm{F}$, et al. Inflammatory monocytes regulate pathologic responses to commensals during acute gastrointestinal infection. Nat Med. 2013;19(6):713-21. PMCID: 3755478.

85. $\mathrm{Yu}$ Y, Chadee K. Prostaglandin E2 stimulates IL-8 gene expression in human colonic epithelial cells by a posttranscriptional mechanism. J Immunol. 1998;161(7):3746-52.

86. Nakayama T, Mutsuga N, Yao L, Tosato G. Prostaglandin E2 promotes degranulation-independent release of MCP-1 from mast cells. J Leukoc Biol. 2006;79(1):95-104.

87. Weller CL, Collington SJ, Hartnell A, Conroy DM, Kaise T, Barker JE, et al. Chemotactic action of prostaglandin E2 on mouse mast cells acting via the 
PGE2 receptor 3. Proc Natl Acad Sci U S A. 2007;104(28):11712-7. PMCID: 1913869.

88. Hu ZQ, Asano K, Seki H, Shimamura T. An essential role of prostaglandin E on mouse mast cell induction. J Immunol. 1995;155(4):2134-42.

89. Snijdewint FG, Kalinski $P$, Wierenga EA, Bos JD, Kapsenberg ML. Prostaglandin E2 differentially modulates cytokine secretion profiles of human $\mathrm{T}$ helper lymphocytes. J Immunol. 1993;150(12):5321-9.

90. Betz M, Fox BS. Prostaglandin E2 inhibits production of Th1 lymphokines but not of Th2 lymphokines. J Immunol. 1991;146(1):108-13.

91. Yao C, Sakata D, Esaki Y, Li Y, Matsuoka T, Kuroiwa K, et al. Prostaglandin E2-EP4 signaling promotes immune inflammation through Th1 cell differentiation and Th17 cell expansion. Nat Med. 2009;15(6):633-40.

92. Kojima F, Kapoor M, Yang L, Fleishaker EL, Ward MR, Monrad SU, et al. Defective generation of a humoral immune response is associated with a reduced incidence and severity of collagen-induced arthritis in microsomal prostaglandin E synthase-1 null mice. J Immunol. 2008;180(12):8361-8. PMCID: 2435291.

93. Sheibanie AF, Khayrullina T, Safadi FF, Ganea D. Prostaglandin E2 exacerbates collagen-induced arthritis in mice through the inflammatory interleukin-23/interleukin-17 axis. Arthritis Rheum. 2007;56(8):2608-19.

94. Gross S, Tilly P, Hentsch D, Vonesch JL, Fabre JE. Vascular wallproduced prostaglandin E2 exacerbates arterial thrombosis and atherothrombosis through platelet EP3 receptors. J Exp Med. 2007;204(2):31120. PMCID: 2118736.

95. Sheibanie AF, Yen JH, Khayrullina T, Emig F, Zhang M, Tuma R, et al. The proinflammatory effect of prostaglandin E2 in experimental inflammatory bowel disease is mediated through the IL-23-->IL-17 axis. J Immunol. 2007;178(12):8138-47.

96. Sugimoto Y, Narumiya S. Prostaglandin E receptors. J Biol Chem. 2007;282(16):11613-7. 
97. Serezani $\mathrm{CH}$, Ballinger MN, Aronoff DM, Peters-Golden M. Cyclic AMP: master regulator of innate immune cell function. Am J Respir Cell Mol Biol. 2008;39(2):127-32. PMCID: 2720142.

98. Ydrenius L, Majeed M, Rasmusson BJ, Stendahl O, Sarndahl E. Activation of cAMP-dependent protein kinase is necessary for actin rearrangements in human neutrophils during phagocytosis. J Leukoc Biol. 2000;67(4):520-8.

99. Zalavary $\mathrm{S}$, Bengtsson T. Adenosine inhibits actin dynamics in human neutrophils: evidence for the involvement of cAMP. Eur $J$ Cell Biol. 1998;75(2):128-39.

100. Mancuso P, Lewis C, Serezani CH, Goel D, Peters-Golden M. Intrapulmonary administration of leukotriene B4 enhances pulmonary host defense against pneumococcal pneumonia. Infect Immun. 2010;78(5):2264-71. PMCID: 2863513.

101. Mancuso P, Nana-Sinkam P, Peters-Golden M. Leukotriene B4 augments neutrophil phagocytosis of Klebsiella pneumoniae. Infect Immun. 2001;69(4):2011-6. PMCID: 98124.

102. Mancuso P, Standiford TJ, Marshall T, Peters-Golden M. 5-Lipoxygenase reaction products modulate alveolar macrophage phagocytosis of Klebsiella pneumoniae. Infect Immun. 1998;66(11):5140-6. PMCID: 108641.

103. Aronoff DM, Bergin IL, Lewis C, Goel D, O'Brien E, Peters-Golden M, et al. E-prostanoid 2 receptor signaling suppresses lung innate immunity against Streptococcus pneumoniae. Prostaglandins Other Lipid Mediat. 2012;98(1-2):2330. PMCID: 3350638.

104. Lee SP, Serezani CH, Medeiros Al, Ballinger MN, Peters-Golden M. Crosstalk between prostaglandin E2 and leukotriene B4 regulates phagocytosis in alveolar macrophages via combinatorial effects on cyclic AMP. J Immunol. 2009;182(1):530-7.

105. Serezani $\mathrm{CH}$, Lewis $\mathrm{C}$, Jancar S, Peters-Golden M. Leukotriene B4 amplifies NF-kappaB activation in mouse macrophages by reducing SOCS1 inhibition of MyD88 expression. J Clin Invest. 2011.

106. Kim ND, Chou RC, Seung E, Tager AM, Luster AD. A unique requirement for the leukotriene B4 receptor BLT1 for neutrophil recruitment in inflammatory arthritis. J Exp Med. 2006;203(4):829-35. PMCID: 2118298. 
107. Costa MF, de Souza-Martins R, de Souza MC, Benjamim CF, Piva B, Diaz $\mathrm{BL}$, et al. Leukotriene B4 mediates gammadelta $\mathrm{T}$ lymphocyte migration in response to diverse stimuli. J Leukoc Biol. 2010;87(2):323-32.

108. Bailie MB, Standiford TJ, Laichalk LL, Coffey MJ, Strieter R, PetersGolden M. Leukotriene-deficient mice manifest enhanced lethality from Klebsiella pneumonia in association with decreased alveolar macrophage phagocytic and bactericidal activities. J Immunol. 1996;157(12):5221-4.

109. Chen M, Lam BK, Kanaoka Y, Nigrovic PA, Audoly LP, Austen KF, et al. Neutrophil-derived leukotriene B4 is required for inflammatory arthritis. J Exp Med. 2006;203(4):837-42. PMCID: 2118292.

110. Chen M, Lam BK, Luster AD, Zarini S, Murphy RC, Bair AM, et al. Joint tissues amplify inflammation and alter their invasive behavior via leukotriene B4 in experimental inflammatory arthritis. J Immunol. 2010;185(9):5503-11.

111. Holgate ST, Peters-Golden M. Introduction: the anti-inflammatory role of cysteinyl leukotriene receptor antagonists in asthma. J Allergy Clin Immunol. 2003;111(1 Suppl):S1-4.

112. Holgate ST, Peters-Golden M, Panettieri RA, Henderson WR, Jr. Roles of cysteinyl leukotrienes in airway inflammation, smooth muscle function, and remodeling. J Allergy Clin Immunol. 2003;111(1 Suppl):S18-34; discussion S-6.

113. lizuka $\mathrm{Y}$, Okuno T, Saeki K, Uozaki H, Okada S, Misaka T, et al. Protective role of the leukotriene B4 receptor BLT2 in murine inflammatory colitis. FASEB J. 2010;24(12):4678-90.

114. Baker N, O'Meara SJ, Scannell M, Maderna P, Godson C. Lipoxin A4: anti-inflammatory and anti-angiogenic impact on endothelial cells. J Immunol. 2009;182(6):3819-26.

115. Levy BD, Kohli $\mathrm{P}$, Gotlinger K, Haworth O, Hong S, Kazani S, et al. Protectin D1 is generated in asthma and dampens airway inflammation and hyperresponsiveness. J Immunol. 2007;178(1):496-502. PMCID: 3005704.

116. Levy BD, Lukacs NW, Berlin AA, Schmidt B, Guilford WJ, Serhan CN, et al. Lipoxin A4 stable analogs reduce allergic airway responses via mechanisms distinct from CysLT1 receptor antagonism. FASEB J. 2007;21(14):3877-84. PMCID: 3005621. 
117. Arita $M$, Ohira $T$, Sun $Y P$, Elangovan $S$, Chiang $N$, Serhan $C N$. Resolvin E1 selectively interacts with leukotriene B4 receptor BLT1 and ChemR23 to regulate inflammation. J Immunol. 2007;178(6):3912-7.

118. (CDC) CfDCaP. CDC - Chronic Disease: Arthritis - At A Glance 2013. [Webpage] Atlanta: Centers for Disease Control and Prevention; 2013 [updated 2013 July 18, 2013; cited 2013 October 22]; Available from: www.cdc.gov/chronicdisease/resources/publications/aag/arthritis.htm.

119. Steiner G, Smolen J. Autoantibodies in rheumatoid arthritis and their clinical significance. Arthritis Res. 2002;4 Suppl 2:S1-5. PMCID: 3238219.

120. Bacon RM, Kugeler KJ, Mead PS. Surveillance for Lyme disease--United States, 1992-2006. MMWR Surveill Summ. 2008;57(10):1-9.

121. Shapiro ED, Gerber MA. Lyme disease. Clin Infect Dis. 2000;31(2):53342.

122. Steere AC, Malawista SE, Snydman DR, Shope RE, Andiman WA, Ross $\mathrm{MR}$, et al. Lyme arthritis: an epidemic of oligoarticular arthritis in children and adults in three connecticut communities. Arthritis Rheum. 1977;20(1):7-17.

123. Burgdorfer W, Barbour AG, Hayes SF, Benach JL, Grunwaldt E, Davis JP. Lyme disease-a tick-borne spirochetosis? Science. 1982;216(4552):1317-9.

124. Murray TS, Shapiro ED. Lyme disease. Clin Lab Med. 2010;30(1):311-28.

125. Barthold SW, Persing DH, Armstrong AL, Peeples RA. Kinetics of Borrelia burgdorferi dissemination and evolution of disease after intradermal inoculation of mice. Am J Pathol. 1991;139(2):263-73. PMCID: 1886084.

126. Armstrong AL, Barthold SW, Persing DH, Beck DS. Carditis in Lyme disease susceptible and resistant strains of laboratory mice infected with Borrelia burgdorferi. Am J Trop Med Hyg. 1992;47(2):249-58.

127. Barthold SW. Antigenic stability of Borrelia burgdorferi during chronic infections of immunocompetent mice. Infect Immun. 1993;61(12):4955-61. PMCID: 281269.

128. Bockenstedt LK, Gonzalez DG, Haberman AM, Belperron AA. Spirochete antigens persist near cartilage after murine Lyme borreliosis therapy. J Clin Invest. 2012;122(7):2652-60. PMCID: 3386809. 
129. Bockenstedt LK, Mao J, Hodzic E, Barthold SW, Fish D. Detection of attenuated, noninfectious spirochetes in Borrelia burgdorferi-infected mice after antibiotic treatment. J Infect Dis. 2002;186(10):1430-7.

130. Barthold SW, Beck DS, Hansen GM, Terwilliger GA, Moody KD. Lyme borreliosis in selected strains and ages of laboratory mice. J Infect Dis. 1990;162(1):133-8.

131. Barthold SW. Infectivity of Borrelia burgdorferi relative to route of inoculation and genotype in laboratory mice. J Infect Dis. 1991;163(2):419-20.

132. Brown CR, Blaho VA, Loiacono CM. Susceptibility to experimental Lyme arthritis correlates with $\mathrm{KC}$ and monocyte chemoattractant protein-1 production in joints and requires neutrophil recruitment via CXCR2. J Immunol. 2003;171(2):893-901.

133. Brown $\mathrm{CR}$, Blaho VA, Loiacono CM. Treatment of mice with the neutrophil-depleting antibody RB6-8C5 results in early development of experimental lyme arthritis via the recruitment of $\mathrm{Gr}$-1- polymorphonuclear leukocyte-like cells. Infect Immun. 2004;72(9):4956-65. PMCID: 517421.

134. Ritzman AM, Hughes-Hanks JM, Blaho VA, Wax LE, Mitchell WJ, Brown CR. The chemokine receptor CXCR2 ligand KC (CXCL1) mediates neutrophil recruitment and is critical for development of experimental Lyme arthritis and carditis. Infect Immun. 2010;78(11):4593-600. PMCID: 2976349.

135. Ruderman EM, Kerr JS, Telford SR, 3rd, Spielman A, Glimcher LH, Gravallese EM. Early murine Lyme carditis has a macrophage predominance and is independent of major histocompatibility complex class II-CD4+ T cell interactions. J Infect Dis. 1995;171(2):362-70.

136. Barthold SW, Feng S, Bockenstedt LK, Fikrig E, Feen K. Protective and arthritis-resolving activity in sera of mice infected with Borrelia burgdorferi. Clin Infect Dis. 1997;25 Suppl 1:S9-17.

137. Barthold SW, Hodzic E, Tunev S, Feng S. Antibody-mediated disease remission in the mouse model of lyme borreliosis. Infect Immun. 2006;74(8):4817-25. PMCID: 1539599.

138. Fikrig E, Barthold SW, Chen M, Chang CH, Flavell RA. Protective antibodies develop, and murine Lyme arthritis regresses, in the absence of MHC class II and CD4+ T cells. J Immunol. 1997;159(11):5682-6. 
139. Fikrig E, Barthold SW, Chen M, Grewal IS, Craft J, Flavell RA. Protective antibodies in murine Lyme disease arise independently of CD40 ligand. J Immunol. 1996;157(1):1-3.

140. Anguita J, Samanta S, Ananthanarayanan SK, Revilla B, Geba GP, Barthold SW, et al. Cyclooxygenase 2 activity modulates the severity of murine Lyme arthritis. FEMS Immunol Med Microbiol. 2002;34(3):187-91.

141. Blaho VA, Mitchell WJ, Brown CR. Arthritis develops but fails to resolve during inhibition of cyclooxygenase 2 in a murine model of Lyme disease. Arthritis Rheum. 2008;58(5):1485-95.

142. Blaho VA, Zhang Y, Hughes-Hanks JM, Brown CR. 5-Lipoxygenasedeficient mice infected with Borrelia burgdorferi develop persistent arthritis. J Immunol. 2011;186(5):3076-84. PMCID: 3346295.

143. Blaho VA, Buczynski MW, Dennis EA, Brown CR. Cyclooxygenase-1 orchestrates germinal center formation and antibody class-switch via regulation of IL-17. J Immunol. 2009;183(9):5644-53.

144. Blaho VA, Buczynski MW, Brown CR, Dennis EA. Lipidomic analysis of dynamic eicosanoid responses during the induction and resolution of Lyme arthritis. J Biol Chem. 2009;284(32):21599-612. PMCID: 2755884.

145. Dumlao DS, Cunningham AM, Wax LE, Norris PC, Hanks JH, Halpin R, et al. Dietary Fish Oil Substitution Alters the Eicosanoid Profile in Ankle Joints of Mice during Lyme Infection. J Nutr. 2012;142(8):1582-9. PMCID: 3397342.

146. Reeves PG, Nielsen FH, Fahey GC, Jr. AIN-93 purified diets for laboratory rodents: final report of the American Institute of Nutrition ad hoc writing committee on the reformulation of the AIN-76A rodent diet. $J$ Nutr. 1993;123(11):1939-51.

147. Reeves PG, Rossow KL, Lindlauf J. Development and testing of the AIN93 purified diets for rodents: results on growth, kidney calcification and bone mineralization in rats and mice. J Nutr. 1993;123(11):1923-31.

148. King VL, Trivedi DB, Gitlin JM, Loftin CD. Selective cyclooxygenase-2 inhibition with celecoxib decreases angiotensin II-induced abdominal aortic aneurysm formation in mice. Arterioscler Thromb Vasc Biol. 2006;26(5):1137-43. 
149. Fritsche KL, Anderson M, Feng C. Consumption of eicosapentaenoic acid and docosahexaenoic acid impair murine interleukin-12 and interferon-gamma production in vivo. J Infect Dis. 2000;182 Suppl 1:S54-61.

150. Huang SC, Misfeldt ML, Fritsche KL. Dietary fat influences la antigen expression and immune cell populations in the murine peritoneum and spleen. $\mathrm{J}$ Nutr. 1992;122(6):1219-31.

151. Barbour AG. Isolation and cultivation of Lyme disease spirochetes. Yale J Biol Med. 1984;57(4):521-5. PMCID: 2589996.

152. de Souza MS, Fikrig E, Smith AL, Flavell RA, Barthold SW. Nonspecific proliferative responses of murine lymphocytes to Borrelia burgdorferi antigens. J Infect Dis. 1992;165(3):471-8.

153. Anguita J, Persing DH, Rincon M, Barthold SW, Fikrig E. Effect of antiinterleukin 12 treatment on murine lyme borreliosis. $J$ Clin Invest. 1996;97(4):1028-34. PMCID: 507149.

154. Monach P, Hattori K, Huang H, Hyatt E, Morse J, Nguyen L, et al. The $\mathrm{K} / \mathrm{BxN}$ mouse model of inflammatory arthritis: theory and practice. Methods Mol Med. 2007;136:269-82.

155. Morrison TB, Ma Y, Weis JH, Weis JJ. Rapid and sensitive quantification of Borrelia burgdorferi-infected mouse tissues by continuous fluorescent monitoring of PCR. J Clin Microbiol. 1999;37(4):987-92. PMCID: 88637.

156. Yang L, Weis JH, Eichwald E, Kolbert CP, Persing DH, Weis JJ. Heritable susceptibility to severe Borrelia burgdorferi-induced arthritis is dominant and is associated with persistence of large numbers of spirochetes in tissues. Infect Immun. 1994;62(2):492-500. PMCID: 186134.

157. Pahl A, Kuhlbrandt U, Brune K, Rollinghoff M, Gessner A. Quantitative detection of Borrelia burgdorferi by real-time PCR. J Clin Microbiol. 1999;37(6):1958-63. PMCID: 84995.

158. Dumlao DS, Buczynski MW, Norris PC, Harkewicz R, Dennis EA. Highthroughput lipidomic analysis of fatty acid derived eicosanoids and $\mathrm{N}$ acylethanolamines. Biochim Biophys Acta. 2011;1811(11):724-36. PMCID: 3205334. 
159. de Souza MS, Smith AL, Beck DS, Terwilliger GA, Fikrig E, Barthold SW. Long-term study of cell-mediated responses to Borrelia burgdorferi in the laboratory mouse. Infect Immun. 1993;61(5):1814-22. PMCID: 280770.

160. McKisic MD, Barthold SW. T-cell-independent responses to Borrelia burgdorferi are critical for protective immunity and resolution of lyme disease. Infect Immun. 2000;68(9):5190-7. PMCID: 101777.

161. Schrier D, Gilbertsen RB, Lesch M, Fantone J. The role of neutrophils in type II collagen-induced arthritis in rats. Am J Pathol. 1984;117(1):26-9. PMCID: 1900570.

162. Zhou JS, Xing W, Friend DS, Austen KF, Katz HR. Mast cell deficiency in Kit(W-sh) mice does not impair antibody-mediated arthritis. J Exp Med. 2007;204(12):2797-802. PMCID: 2118523.

163. Wipke BT, Allen PM. Essential role of neutrophils in the initiation and progression of a murine model of rheumatoid arthritis. J Immunol. 2001;167(3):1601-8.

164. Monach PA, Nigrovic PA, Chen M, Hock H, Lee DM, Benoist C, et al. Neutrophils in a mouse model of autoantibody-mediated arthritis: critical producers of $\mathrm{Fc}$ receptor gamma, the receptor for $\mathrm{C} 5 \mathrm{a}$, and lymphocyte functionassociated antigen 1. Arthritis Rheum. 2010;62(3):753-64. PMCID: 3057458.

165. Santos LL, Morand EF, Hutchinson P, Boyce NW, Holdsworth SR. Antineutrophil monoclonal antibody therapy inhibits the development of adjuvant arthritis. Clin Exp Immunol. 1997;107(2):248-53. PMCID: 1904585.

166. Schrier DJ, Schimmer RC, Flory CM, Tung DK, Ward PA. Role of chemokines and cytokines in a reactivation model of arthritis in rats induced by injection with streptococcal cell walls. J Leukoc Biol. 1998;63(3):359-63.

167. Pillinger $\mathrm{MH}$, Abramson SB. The neutrophil in rheumatoid arthritis. Rheum Dis Clin North Am. 1995;21(3):691-714.

168. Weissmann G, Korchak H. Rheumatoid arthritis. The role of neutrophil activation. Inflammation. 1984;8 Suppl:S3-14.

169. Morrison TB, Weis $\mathrm{JH}$, Weis JJ. Borrelia burgdorferi outer surface protein A (OspA) activates and primes human neutrophils. J Immunol. 1997;158(10):4838-45. 
170. Lusitani D, Malawista SE, Montgomery RR. Calprotectin, an abundant cytosolic protein from human polymorphonuclear leukocytes, inhibits the growth of Borrelia burgdorferi. Infect Immun. 2003;71(8):4711-6. PMCID: 166021.

171. Xu Q, Seemanapalli SV, Reif KE, Brown CR, Liang FT. Increasing the recruitment of neutrophils to the site of infection dramatically attenuates Borrelia burgdorferi infectivity. J Immunol. 2007;178(8):5109-15.

172. Cassatella MA. Neutrophil-derived proteins: selling cytokines by the pound. Adv Immunol. 1999;73:369-509.

173. Amulic B, Cazalet C, Hayes GL, Metzler KD, Zychlinsky A. Neutrophil Function: From Mechanisms to Disease. Annu Rev Immunol. 2011.

174. Basran A, Jabeen M, Bingle L, Stokes CA, Dockrell DH, Whyte MK, et al. Roles of neutrophils in the regulation of the extent of human inflammation through delivery of IL-1 and clearance of chemokines. J Leukoc Biol. 2013;93(1):7-19. PMCID: 3525835.

175. Borregaard N. Neutrophils, from marrow to microbes. Immunity. 2010;33(5):657-70.

176. Coffey MJ, Phare SM, Peters-Golden M. Role of leukotrienes in killing of Mycobacterium bovis by neutrophils. Prostaglandins Leukot Essent Fatty Acids. 2004;71(3):185-90.

177. Kumar V, Sharma A. Neutrophils: Cinderella of innate immune system. Int Immunopharmacol. 2010;10(11):1325-34.

178. Motameni AR, Bates TC, Juncadella IJ, Petty C, Hedrick MN, Anguita J. Distinct bacterial dissemination and disease outcome in mice subcutaneously infected with Borrelia burgdorferi in the midline of the back and the footpad. FEMS Immunol Med Microbiol. 2005;45(2):279-84.

179. Montgomery RR, Lusitani D, de Boisfleury Chevance A, Malawista SE. Human phagocytic cells in the early innate immune response to Borrelia burgdorferi. J Infect Dis. 2002;185(12):1773-9.

180. Hawley KL, Olson CM, Jr., Iglesias-Pedraz JM, Navasa N, Cervantes JL, Caimano MJ, et al. CD14 cooperates with complement receptor 3 to mediate MyD88-independent phagocytosis of Borrelia burgdorferi. Proc Natl Acad Sci U S A. 2012;109(4):1228-32. PMCID: 3268315. 
181. Mackay CR. Moving targets: cell migration inhibitors as new antiinflammatory therapies. Nat Immunol. 2008;9(9):988-98.

182. Bizzarri C, Beccari AR, Bertini R, Cavicchia MR, Giorgini S, Allegretti M. ELR+ CXC chemokines and their receptors (CXC chemokine receptor 1 and CXC chemokine receptor 2) as new therapeutic targets. Pharmacol Ther. 2006;112(1):139-49.

183. Koch $A E$. Chemokines and their receptors in rheumatoid arthritis: future targets? Arthritis Rheum. 2005;52(3):710-21.

184. Szekanecz Z, Koch AE. Vascular involvement in rheumatic diseases: 'vascular rheumatology'. Arthritis Res Ther. 2008;10(5):224. PMCID: 2592799.

185. Vergunst CE, Gerlag DM, Lopatinskaya L, Klareskog L, Smith MD, van den Bosch F, et al. Modulation of CCR2 in rheumatoid arthritis: a double-blind, randomized, placebo-controlled clinical trial. Arthritis Rheum. 2008;58(7):1931-9.

186. Montgomery RR, Nathanson MH, Malawista SE. The fate of Borrelia burgdorferi, the agent for Lyme disease, in mouse macrophages. Destruction, survival, recovery. J Immunol. 1993;150(3):909-15.

187. Montgomery RR, Nathanson MH, Malawista SE. Fc- and non-Fc-mediated phagocytosis of Borrelia burgdorferi by macrophages. J Infect Dis. 1994;170(4):890-3.

188. Codolo G, Amedei A, Steere AC, Papinutto E, Cappon A, Polenghi A, et al. Borrelia burgdorferi NapA-driven Th17 cell inflammation in lyme arthritis. Arthritis Rheum. 2008;58(11):3609-17.

189. Md Yusof MY, Emery P. Targeting interleukin-6 in rheumatoid arthritis. Drugs. 2013;73(4):341-56.

190. Geiler J, Buch M, McDermott MF. Anti-TNF treatment in rheumatoid arthritis. Curr Pharm Des. 2011;17(29):3141-54.

191. Abramson SB, Amin A. Blocking the effects of IL-1 in rheumatoid arthritis protects bone and cartilage. Rheumatology (Oxford). 2002;41(9):972-80.

192. Morimoto K, Shirata N, Taketomi Y, Tsuchiya S, Segi-Nishida E, Inazumi $\mathrm{T}$, et al. Prostaglandin E2-EP3 signaling induces inflammatory swelling by mast cell activation. J Immunol. 2014;192(3):1130-7. 
193. Dorris SL, Peebles RS, Jr. PGI2 as a regulator of inflammatory diseases. Mediators Inflamm. 2012;2012:926968. PMCID: 3407649.

194. Regan JW. EP2 and EP4 prostanoid receptor signaling. Life Sci. 2003;74(2-3):143-53.

195. Fedyk ER, Phipps RP. Prostaglandin E2 receptors of the EP2 and EP4 subtypes regulate activation and differentiation of mouse B lymphocytes to IgEsecreting cells. Proc Natl Acad Sci U S A. 1996;93(20):10978-83. PMCID: 38269.

196. Lin CR, Amaya F, Barrett L, Wang H, Takada J, Samad TA, et al. Prostaglandin E2 receptor EP4 contributes to inflammatory pain hypersensitivity. J Pharmacol Exp Ther. 2006;319(3):1096-103.

197. Zimecki M. Potential therapeutic interventions via EP2/EP4 prostaglandin receptors. Postepy Hig Med Dosw (Online). 2012;66:287-94.

198. Shinomiya S, Naraba H, Ueno A, Utsunomiya I, Maruyama T, Ohuchida S, et al. Regulation of TNFalpha and interleukin-10 production by prostaglandins $\mathrm{I}(2)$ and $E(2)$ : studies with prostaglandin receptor-deficient mice and prostaglandin E-receptor subtype-selective synthetic agonists. Biochem Pharmacol. 2001;61(9):1153-60.

199. Ratcliffe MJ, Walding A, Shelton PA, Flaherty A, Dougall IG. Activation of E-prostanoid4 and E-prostanoid2 receptors inhibits TNF-alpha release from human alveolar macrophages. Eur Respir J. 2007;29(5):986-94.

200. Shishodia S, Koul D, Aggarwal BB. Cyclooxygenase (COX)-2 inhibitor celecoxib abrogates TNF-induced NF-kappa B activation through inhibition of activation of I kappa B alpha kinase and Akt in human non-small cell lung carcinoma: correlation with suppression of COX-2 synthesis. J Immunol. 2004;173(3):2011-22.

201. Vassiliou E, Jing H, Ganea D. Prostaglandin E2 inhibits TNF production in murine bone marrow-derived dendritic cells. Cell Immunol. 2003;223(2):120-32.

202. Bouffi C, Bony C, Courties G, Jorgensen C, Noel D. IL-6-dependent PGE2 secretion by mesenchymal stem cells inhibits local inflammation in experimental arthritis. PLoS One. 2010;5(12):e14247. PMCID: 2998425.

203. Chen BC, Liao CC, Hsu MJ, Liao YT, Lin CC, Sheu JR, et al. Peptidoglycan-induced IL-6 production in RAW 264.7 macrophages is mediated 
by cyclooxygenase-2, PGE2/PGE4 receptors, protein kinase A, I kappa B kinase, and NF-kappa B. J Immunol. 2006;177(1):681-93.

204. Treffkorn L, Scheibe R, Maruyama T, Dieter P. PGE2 exerts its effect on the LPS-induced release of TNF-alpha, ET-1, IL-1alpha, IL-6 and IL-10 via the EP2 and EP4 receptor in rat liver macrophages. Prostaglandins Other Lipid Mediat. 2004;74(1-4):113-23.

205. Walch L, Morris PL. Cyclooxygenase 2 pathway mediates IL-1beta regulation of IL-1alpha, -1beta, and IL-6 mRNA levels in Leydig cell progenitors. Endocrinology. 2002;143(9):3276-83.

206. Portanova JP, Zhang Y, Anderson GD, Hauser SD, Masferrer JL, Seibert $\mathrm{K}$, et al. Selective neutralization of prostaglandin E2 blocks inflammation, hyperalgesia, and interleukin 6 production in vivo. J Exp Med. 1996;184(3):88391. PMCID: 2192784.

207. Hunt D, Wilson JE, Weih KA, Ishido S, Harton JA, Roche PA, et al. Francisella tularensis elicits IL-10 via a PGE(2)-inducible factor, to drive macrophage MARCH1 expression and class II down-regulation. PLoS One. 2012;7(5):e37330. PMCID: 3355121.

208. Ramaswamy K, Kumar P, He YX. A role for parasite-induced PGE2 in IL10-mediated host immunoregulation by skin stage schistosomula of Schistosoma mansoni. J Immunol. 2000;165(8):4567-74.

209. Buczynski MW, Dumlao DS, Dennis EA. Thematic Review Series: Proteomics. An integrated omics analysis of eicosanoid biology. J Lipid Res. 2009;50(6):1015-38. PMCID: 2681385.

210. Hyde CA, Missailidis S. Inhibition of arachidonic acid metabolism and its implication on cell proliferation and tumour-angiogenesis. Int Immunopharmacol. 2009;9(6):701-15.

211. Calder PC, Zurier RB. Polyunsaturated fatty acids and rheumatoid arthritis. Curr Opin Clin Nutr Metab Care. 2001;4(2):115-21.

212. Weitz D, Weintraub H, Fisher E, Schwartzbard AZ. Fish oil for the treatment of cardiovascular disease. Cardiol Rev. 2010;18(5):258-63. PMCID: 3217043. 
213. Stirban A, Nandrean S, Gotting C, Tamler R, Pop A, Negrean M, et al. Effects of n-3 fatty acids on macro- and microvascular function in subjects with type 2 diabetes mellitus. Am J Clin Nutr. 2010;91(3):808-13.

214. Al-Turkmani MR, Andersson C, Alturkmani R, Katrangi W, Cluette-Brown $\mathrm{JE}$, Freedman SD, et al. A mechanism accounting for the low cellular level of linoleic acid in cystic fibrosis and its reversal by DHA. J Lipid Res. 2008;49(9):1946-54. PMCID: 2515522.

215. Leslie CA, Gonnerman WA, Ullman MD, Hayes KC, Franzblau C, Cathcart ES. Dietary fish oil modulates macrophage fatty acids and decreases arthritis susceptibility in mice. J Exp Med. 1985;162(4):1336-49. PMCID: 2187871.

216. Lee TH, Mencia-Huerta JM, Shih C, Corey EJ, Lewis RA, Austen KF. Effects of exogenous arachidonic, eicosapentaenoic, and docosahexaenoic acids on the generation of 5-lipoxygenase pathway products by ionophore-activated human neutrophils. J Clin Invest. 1984;74(6):1922-33. PMCID: 425378.

217. Goldman DW, Pickett WC, Goetzl EJ. Human neutrophil chemotactic and degranulating activities of leukotriene B5 (LTB5) derived from eicosapentaenoic acid. Biochem Biophys Res Commun. 1983;117(1):282-8.

218. Lands B. A critique of paradoxes in current advice on dietary lipids. Prog Lipid Res. 2008;47(2):77-106.

219. Bagga D, Wang L, Farias-Eisner R, Glaspy JA, Reddy ST. Differential effects of prostaglandin derived from omega- 6 and omega- 3 polyunsaturated fatty acids on COX-2 expression and IL-6 secretion. Proc Natl Acad Sci U S A. 2003;100(4):1751-6. PMCID: 149905.

220. Komatsu W, Ishihara K, Murata M, Saito H, Shinohara K. Docosahexaenoic acid suppresses nitric oxide production and inducible nitric oxide synthase expression in interferon-gamma plus lipopolysaccharidestimulated murine macrophages by inhibiting the oxidative stress. Free Radic Biol Med. 2003;34(8):1006-16.

221. Yan Y, Jiang W, Spinetti T, Tardivel A, Castillo R, Bourquin C, et al. Omega-3 fatty acids prevent inflammation and metabolic disorder through inhibition of NLRP3 inflammasome activation. Immunity. 2013;38(6):1154-63.

222. Steere AC, Glickstein L. Elucidation of Lyme arthritis. Nat Rev Immunol. 2004;4(2):143-52. 
223. Burhop KE, Selig WM, Malik AB. Monohydroxyeicosatetraenoic acids (5HETE and 15-HETE) induce pulmonary vasoconstriction and edema. Circ Res. 1988;62(4):687-98.

224. Divanovic S, Dalli J, Jorge-Nebert LF, Flick LM, Galvez-Peralta M, Boespflug ND, et al. Contributions of the Three CYP1 Monooxygenases to ProInflammatory and Inflammation-Resolution Lipid Mediator Pathways. J Immunol. 2013.

225. Dalli J, Colas RA, Serhan CN. Novel n-3 immunoresolvents: structures and actions. Sci Rep. 2013;3:1940. PMCID: 3672887.

226. Capdevila JH, Falck JR. Biochemical and molecular properties of the cytochrome P450 arachidonic acid monooxygenases. Prostaglandins Other Lipid Mediat. 2002;68-69:325-44.

227. Vincent C, Fiancette R, Donnard M, Bordessoule D, Turlure P, Trimoreau $\mathrm{F}$, et al. 5-LOX, 12-LOX and 15-LOX in immature forms of human leukemic blasts. Leuk Res. 2008;32(11):1756-62.

228. Moreno JJ. New aspects of the role of hydroxyeicosatetraenoic acids in cell growth and cancer development. Biochem Pharmacol. 2009;77(1):1-10.

229. Mesaros C, Lee SH, Blair IA. Targeted quantitative analysis of eicosanoid lipids in biological samples using liquid chromatography-tandem mass spectrometry. J Chromatogr B Analyt Technol Biomed Life Sci. 2009;877(26):2736-45. PMCID: 2745066.

230. Gong Y, Yi M, Fediuk J, Lizotte PP, Dakshinamurti S. Hypoxic neonatal pulmonary arterial myocytes are sensitized to ROS-generated 8-isoprostane. Free Radic Biol Med. 2010;48(7):882-94.

231. Montuschi $P$, Corradi $M$, Ciabattoni G, Nightingale J, Kharitonov SA, Barnes PJ. Increased 8-isoprostane, a marker of oxidative stress, in exhaled condensate of asthma patients. Am J Respir Crit Care Med. 1999;160(1):216-20.

232. Stables MJ, Gilroy DW. Old and new generation lipid mediators in acute inflammation and resolution. Prog Lipid Res. 2011;50(1):35-51.

233. Wada M, DeLong CJ, Hong YH, Rieke CJ, Song I, Sidhu RS, et al. Enzymes and receptors of prostaglandin pathways with arachidonic acid-derived 
versus eicosapentaenoic acid-derived substrates and products. J Biol Chem. 2007;282(31):22254-66.

234. Corey EJ, Shih C, Cashman JR. Docosahexaenoic acid is a strong inhibitor of prostaglandin but not leukotriene biosynthesis. Proc Natl Acad Sci U S A. 1983;80(12):3581-4. PMCID: 394093.

235. Norris PC, Dennis EA. Omega-3 fatty acids cause dramatic changes in TLR4 and purinergic eicosanoid signaling. Proc Natl Acad Sci U S A. 2012;109(22):8517-22. PMCID: 3365225.

236. Bourdonnay E, Serezani CH, Aronoff DM, Peters-Golden M. Regulation of alveolar macrophage p40phox: hierarchy of activating kinases and their inhibition by PGE2. J Leukoc Biol. 2012;92(1):219-31. PMCID: 3382311.

237. Chen M, Divangahi M, Gan H, Shin DS, Hong S, Lee DM, et al. Lipid mediators in innate immunity against tuberculosis: opposing roles of PGE2 and LXA4 in the induction of macrophage death. J Exp Med. 2008;205(12):2791-801. PMCID: 2585850.

238. Downey GP, Gumbay RS, Doherty DE, LaBrecque JF, Henson JE, Henson PM, et al. Enhancement of pulmonary inflammation by PGE2: evidence for a vasodilator effect. J Appl Physiol. 1988;64(2):728-41.

239. Kreydiyyeh SI, Riman S, Serhan M, Kassardjian A. TNF-alpha modulates hepatic Na+-K+ ATPase activity via PGE2 and EP2 receptors. Prostaglandins Other Lipid Mediat. 2007;83(4):295-303.

240. Weinlich R, Bortoluci KR, Chehab CF, Serezani CH, Ulbrich AG, PetersGolden M, et al. TLR4/MYD88-dependent, LPS-induced synthesis of PGE2 by macrophages or dendritic cells prevents anti-CD3-mediated CD95L upregulation in T cells. Cell Death Differ. 2008;15(12):1901-9.

241. Rajakariar R, Hilliard M, Lawrence T, Trivedi S, Colville-Nash P, Bellingan $\mathrm{G}$, et al. Hematopoietic prostaglandin D2 synthase controls the onset and resolution of acute inflammation through PGD2 and 15-deoxyDelta12 14 PGJ2. Proc Natl Acad Sci U S A. 2007;104(52):20979-84. PMCID: 2409252.

242. Straus DS, Pascual G, Li M, Welch JS, Ricote M, Hsiang CH, et al. 15deoxy-delta 12,14-prostaglandin J2 inhibits multiple steps in the NF-kappa B signaling pathway. Proc Natl Acad Sci U S A. 2000;97(9):4844-9. PMCID: 18320. 
243. Szanto A, Nagy L. The many faces of PPARgamma: anti-inflammatory by any means? Immunobiology. 2008;213(9-10):789-803.

244. Brown CR, Lai AY, Callen ST, Blaho VA, Hughes JM, Mitchell WJ. Adenoviral delivery of interleukin-10 fails to attenuate experimental Lyme disease. Infect Immun. 2008;76(12):5500-7. PMCID: 2583579.

245. Marques AR. Lyme disease: a review. Curr Allergy Asthma Rep. 2010;10(1):13-20.

246. Fritsche K. Important differences exist in the dose-response relationship between diet and immune cell fatty acids in humans and rodents. Lipids. 2007;42(11):961-79.

247. Schuchardt JP, Schneider I, Meyer H, Neubronner J, von Schacky C, Hahn A. Incorporation of EPA and DHA into plasma phospholipids in response to different omega-3 fatty acid formulations--a comparative bioavailability study of fish oil vs. krill oil. Lipids Health Dis. 2011;10:145. PMCID: 3168413.

248. Oosting M, Buffen K, Malireddi SR, Sturm P, Verschueren I, Koenders MI, et al. Murine Borrelia arthritis is highly dependent on ASC and caspase-1, but independent of NLRP3. Arthritis Res Ther. 2012;14(6):R247. PMCID: 3674595.

249. Harter L, Straubinger RK, Summers BA, Erb HN, Appel MJ. Up-regulation of inducible nitric oxide synthase mRNA in dogs experimentally infected with Borrelia burgdorferi. Vet Immunol Immunopathol. 1999;67(3):271-84.

250. Brown CR, Reiner SL. Development of lyme arthritis in mice deficient in inducible nitric oxide synthase. J Infect Dis. 1999;179(6):1573-6.

251. Bosco N, Brahmbhatt V, Oliveira M, Martin FP, Lichti P, Raymond F, et al. Effects of increase in fish oil intake on intestinal eicosanoids and inflammation in a mouse model of colitis. Lipids Health Dis. 2013;12(1):81. PMCID: 3691874.

252. Kouskoff V, Korganow AS, Duchatelle V, Degott C, Benoist C, Mathis D. Organ-specific disease provoked by systemic autoimmunity. Cell. 1996;87(5):811-22.

253. Korganow AS, Ji H, Mangialaio S, Duchatelle V, Pelanda R, Martin T, et al. From systemic $T$ cell self-reactivity to organ-specific autoimmune disease via immunoglobulins. Immunity. 1999;10(4):451-61. 
254. Mangialaio S, Ji H, Korganow AS, Kouskoff $\mathrm{V}$, Benoist C, Mathis D. The arthritogenic $\mathrm{T}$ cell receptor and its ligand in a model of spontaneous arthritis. Arthritis Rheum. 1999;42(12):2517-23.

255. Ji H, Ohmura K, Mahmood U, Lee DM, Hofhuis FM, Boackle SA, et al. Arthritis critically dependent on innate immune system players. Immunity. 2002;16(2):157-68.

256. Matsumoto I, Maccioni M, Lee DM, Maurice M, Simmons B, Brenner M, et al. How antibodies to a ubiquitous cytoplasmic enzyme may provoke jointspecific autoimmune disease. Nat Immunol. 2002;3(4):360-5.

257. Ji H, Gauguier D, Ohmura K, Gonzalez A, Duchatelle V, Danoy P, et al. Genetic influences on the end-stage effector phase of arthritis. J Exp Med. 2001;194(3):321-30. PMCID: 2193468.

258. Elliott ER, Van Ziffle JA, Scapini P, Sullivan BM, Locksley RM, Lowell CA. Deletion of Syk in neutrophils prevents immune complex arthritis. J Immunol. 2011;187(8):4319-30. PMCID: 3186826.

259. Chen M, Boilard E, Nigrovic PA, Clark P, Xu D, Fitzgerald GA, et al. Predominance of cyclooxygenase 1 over cyclooxygenase 2 in the generation of proinflammatory prostaglandins in autoantibody-driven $\mathrm{K} / \mathrm{BxN}$ serum-transfer arthritis. Arthritis Rheum. 2008;58(5):1354-65.

260. Fabre JE, Goulet JL, Riche E, Nguyen M, Coggins K, Offenbacher S, et al. Transcellular biosynthesis contributes to the production of leukotrienes during inflammatory responses in vivo. J Clin Invest. 2002;109(10):1373-80. PMCID: 150978.

261. Chou RC, Kim ND, Sadik CD, Seung E, Lan Y, Byrne MH, et al. Lipidcytokine-chemokine cascade drives neutrophil recruitment in a murine model of inflammatory arthritis. Immunity. 2010;33(2):266-78. PMCID: 3155777.

262. Sadik CD, Kim ND, Iwakura Y, Luster AD. Neutrophils orchestrate their own recruitment in murine arthritis through $\mathrm{C} 5 \mathrm{aR}$ and FcgammaR signaling. Proc Natl Acad Sci U S A. 2012;109(46):E3177-85. PMCID: 3503206.

263. Mathis SP, Jala VR, Lee DM, Haribabu B. Nonredundant roles for leukotriene B4 receptors BLT1 and BLT2 in inflammatory arthritis. J Immunol. 2010;185(5):3049-56. 
264. Monach PA, Mathis D, Benoist C. The K/BxN arthritis model. Curr Protoc Immunol. 2008;Chapter 15:Unit 1522.

265. Capdevila JH, Falck JR, Harris RC. Cytochrome P450 and arachidonic acid bioactivation. Molecular and functional properties of the arachidonate monooxygenase. J Lipid Res. 2000;41(2):163-81.

266. Zou AP, Fleming JT, Falck JR, Jacobs ER, Gebremedhin D, Harder DR, et al. 20-HETE is an endogenous inhibitor of the large-conductance $\mathrm{Ca}(2+)$ activated K+ channel in renal arterioles. Am J Physiol. 1996;270(1 Pt 2):R228-37.

267. Zhang Y, Oltman CL, Lu T, Lee HC, Dellsperger KC, VanRollins M. EET homologs potently dilate coronary microvessels and activate $\mathrm{BK}(\mathrm{Ca})$ channels. Am J Physiol Heart Circ Physiol. 2001;280(6):H2430-40.

268. Campbell WB, Gebremedhin D, Pratt PF, Harder DR. Identification of epoxyeicosatrienoic acids as endothelium-derived hyperpolarizing factors. Circ Res. 1996;78(3):415-23.

269. Sanada H, Sekimoto M, Kamoshita A, Degawa M. Changes in expression of hepatic cytochrome P450 subfamily enzymes during development of adjuvantinduced arthritis in rats. J Toxicol Sci. 2011;36(2):181-90.

270. Yen JH, Tsai WC, Lin CH, Ou TT, Hu CJ, Liu HW. Manganese superoxide dismutase gene polymorphisms in psoriatic arthritis. Dis Markers. 2003;19(6):263-5.

271. Yen JH, Tsai WC, Lin CH, Ou TT, Hu CJ, Liu HW. Cytochrome P450 1A1 and manganese superoxide dismutase genes polymorphisms in reactive arthritis. Immunol Lett. 2003;90(2-3):151-4.

272. Yen JH, Tsai WC, Chen CJ, Lin CH, Ou TT, Hu CJ, et al. Cytochrome P450 1A1 and manganese superoxide dismutase genes polymorphisms in ankylosing spondylitis. Immunol Lett. 2003;88(2):113-6.

273. Yen JH, Chen CJ, Tsai WC, Lin CH, Ou TT, Hu CJ, et al. Manganese superoxide dismutase and cytochrome P450 1A1 genes polymorphisms in rheumatoid arthritis in Taiwan. Hum Immunol. 2003;64(3):366-73.

274. Campbell WB, Harder DR. Endothelium-derived hyperpolarizing factors and vascular cytochrome P450 metabolites of arachidonic acid in the regulation of tone. Circ Res. 1999;84(4):484-8. 
275. Liu JY, Li N, Yang J, Qiu H, Ai D, Chiamvimonvat N, et al. Metabolic profiling of murine plasma reveals an unexpected biomarker in rofecoxibmediated cardiovascular events. Proc Natl Acad Sci U S A. 2010;107(39):1701722. PMCID: 2947894.

276. McGiff JC. Cytochrome P-450 metabolism of arachidonic acid. Annu Rev Pharmacol Toxicol. 1991;31:339-69.

277. Makita K, Falck JR, Capdevila JH. Cytochrome P450, the arachidonic acid cascade, and hypertension: new vistas for an old enzyme system. FASEB J. 1996;10(13):1456-63.

278. Harris RC, Homma T, Jacobson HR, Capdevila J. Epoxyeicosatrienoic acids activate $\mathrm{Na}+/ \mathrm{H}+$ exchange and are mitogenic in cultured rat glomerular mesangial cells. J Cell Physiol. 1990;144(3):429-37.

279. Node K, Huo Y, Ruan X, Yang B, Spiecker M, Ley K, et al. Antiinflammatory properties of cytochrome P450 epoxygenase-derived eicosanoids. Science. 1999;285(5431):1276-9. PMCID: 2720027.

280. Liu W, Morrow JD, Yin H. Quantification of F2-isoprostanes as a reliable index of oxidative stress in vivo using gas chromatography-mass spectrometry (GC-MS) method. Free Radic Biol Med. 2009;47(8):1101-7. PMCID: 2749920.

281. Basu S, Whiteman M, Mattey DL, Halliwell B. Raised levels of $F(2)$ isoprostanes and prostaglandin $\mathrm{F}(2 \mathrm{alpha})$ in different rheumatic diseases. Ann Rheum Dis. 2001;60(6):627-31. PMCID: 1753663.

282. Szuldrzynski K, Zalewski J, Machnik A, Zmudka K. Elevated levels of 8iso-prostaglandin F2alpha in acute coronary syndromes are associated with systemic and local platelet activation. Pol Arch Med Wewn. 2010;120(1-2):19-24.

283. Brines R, Maicas N, Ferrandiz ML, Loboda A, Jozkowicz A, Dulak J, et al. Heme oxygenase-1 regulates the progression of K/BxN serum transfer arthritis. PLoS One. 2012;7(12):e52435. PMCID: 3527514.

284. Ackerman WEt, Zhang XL, Rovin BH, Kniss DA. Modulation of cytokineinduced cyclooxygenase 2 expression by PPARG ligands through NFkappaB signal disruption in human WISH and amnion cells. Biol Reprod. 2005;73(3):52735. PMCID: 1360652. 
285. Anderson GD, Hauser SD, McGarity KL, Bremer ME, Isakson PC, Gregory SA. Selective inhibition of cyclooxygenase (COX)-2 reverses inflammation and expression of COX-2 and interleukin 6 in rat adjuvant arthritis. J Clin Invest. 1996;97(11):2672-9. PMCID: 507355.

286. Colville-Nash PR, Gilroy DW. Cyclooxygenase enzymes as targets for therapeutic intervention in inflammation. Drug News Perspect. 2000;13(10):58797.

287. Gilroy DW, Tomlinson A, Willoughby DA. Differential effects of inhibitors of cyclooxygenase (cyclooxygenase 1 and cyclooxygenase 2) in acute inflammation. Eur J Pharmacol. 1998;355(2-3):211-7.

288. Fredenburgh LE, Velandia MM, Ma J, Olszak T, Cernadas M, Englert JA, et al. Cyclooxygenase-2 deficiency leads to intestinal barrier dysfunction and increased mortality during polymicrobial sepsis. J Immunol. 2011;187(10):525567. PMCID: 3208135.

289. Harris RC. Cyclooxygenase-2 and the kidney: functional and pathophysiological implications. J Hypertens Suppl. 2002;20(6):S3-9.

290. Honda T, Segi-Nishida E, Miyachi Y, Narumiya S. Prostacyclin-IP signaling and prostaglandin E2-EP2/EP4 signaling both mediate joint inflammation in mouse collagen-induced arthritis. J Exp Med. 2006;203(2):32535. PMCID: 2118213.

291. Sadik CD, Luster AD. Lipid-cytokine-chemokine cascades orchestrate leukocyte recruitment in inflammation. J Leukoc Biol. 2012;91(2):207-15. PMCID: 3290425.

292. Okuno T, lizuka Y, Okazaki H, Yokomizo T, Taguchi R, Shimizu T. 12(S)Hydroxyheptadeca-5Z, 8E, 10E-trienoic acid is a natural ligand for leukotriene B4 receptor 2. J Exp Med. 2008;205(4):759-66. PMCID: 2292216.

293. St-Onge M, Flamand N, Biarc J, Picard S, Bouchard L, Dussault AA, et al. Characterization of prostaglandin E2 generation through the cyclooxygenase (COX)-2 pathway in human neutrophils. Biochim Biophys Acta. 2007;1771(9):1235-45. PMCID: 2891965.

294. Trebino CE, Stock JL, Gibbons CP, Naiman BM, Wachtmann TS, Umland $\mathrm{JP}$, et al. Impaired inflammatory and pain responses in mice lacking an inducible prostaglandin E synthase. Proc Natl Acad Sci U S A. 2003;100(15):9044-9. PMCID: 166435. 
295. Frolov A, Yang L, Dong H, Hammock BD, Crofford LJ. Anti-inflammatory properties of prostaglandin E2: Deletion of microsomal prostaglandin E synthase1 exacerbates non-immune inflammatory arthritis in mice. Prostaglandins Leukot Essent Fatty Acids. 2013.

296. Colli S, Caruso D, Stragliotto E, Morazzoni G, Aletti A, Galli G, et al. Proinflammatory lipoxygenase products from peripheral mononuclear cells in patients with rheumatoid arthritis. J Lab Clin Med. 1988;112(3):357-62.

297. Bittleman DB, Casale TB. 5-Hydroxyeicosatetraenoic acid (HETE)induced neutrophil transcellular migration is dependent upon enantiomeric structure. Am J Respir Cell Mol Biol. 1995;12(3):260-7.

298. Kronke G, Katzenbeisser J, Uderhardt S, Zaiss MM, Scholtysek C, Schabbauer G, et al. 12/15-lipoxygenase counteracts inflammation and tissue damage in arthritis. J Immunol. 2009;183(5):3383-9.

299. Kasuga K, Yang R, Porter TF, Agrawal N, Petasis NA, Irimia D, et al. Rapid appearance of resolvin precursors in inflammatory exudates: novel mechanisms in resolution. J Immunol. 2008;181(12):8677-87. PMCID: 2664686.

300. Tam VC, Quehenberger O, Oshansky CM, Suen R, Armando AM, Treuting PM, et al. Lipidomic profiling of influenza infection identifies mediators that induce and resolve inflammation. Cell. 2013;154(1):213-27. PMCID: 3753192 .

301. Bryn T, Yaqub S, Mahic M, Henjum K, Aandahl EM, Tasken K. LPSactivated monocytes suppress T-cell immune responses and induce FOXP3+ T cells through a COX-2-PGE2-dependent mechanism. Int Immunol. 2008;20(2):235-45.

302. Breyer RM, Bagdassarian CK, Myers SA, Breyer MD. Prostanoid receptors: subtypes and signaling. Annu Rev Pharmacol Toxicol. 2001;41:66190.

303. Steere AC, Hardin JA, Malawista SE. Erythema chronicum migrans and Lyme arthritis: cryoimmunoglobulins and clinical activity of skin and joints. Science. 1977;196(4294):1121-2.

304. Steere AC, Hardin JA, Malawista SE. Erythema chronicum migrans and Lyme arthritis: related problems recently recognized in Connecticut. Conn Med. 1978;42(6):353-7. 
305. Steere AC, Hardin JA, Malawista SE. Lyme arthritis: a new clinical entity. Hosp Pract. 1978;13(4):143-58.

306. Steere AC, Malawista SE, Hardin JA, Ruddy S, Askenase W, Andiman WA. Erythema chronicum migrans and Lyme arthritis. The enlarging clinical spectrum. Ann Intern Med. 1977;86(6):685-98.

307. Steere AC, Broderick TF, Malawista SE. Erythema chronicum migrans and Lyme arthritis: epidemiologic evidence for a tick vector. Am J Epidemiol. 1978;108(4):312-21.

308. Moody KD, Barthold SW, Terwilliger GA. Lyme borreliosis in laboratory animals: effect of host species and in vitro passage of Borrelia burgdorferi. Am J Trop Med Hyg. 1990;43(1):87-92.

309. Barthold SW, de Souza M. Exacerbation of Lyme arthritis in beige mice. J Infect Dis. 1995;172(3):778-84.

310. Alvarez Y, Valera I, Municio C, Hugo E, Padron F, Blanco L, et al. Eicosanoids in the innate immune response: TLR and non-TLR routes. Mediators Inflamm. 2010;2010. PMCID: 2905620.

311. Schwerbrock NM, Karlsson EA, Shi Q, Sheridan PA, Beck MA. Fish oil-fed mice have impaired resistance to influenza infection. J Nutr. 2009;139(8):158894. PMCID: 2709305. 


\section{VITA}

Anna M. Cunningham was born to Paula and Mike Ritzman on December 3, 1985. She has one sister, Mary Margaret (Maggie) Flanagan. She was married on October 8, 2011 to the love of her life, Cody Cunningham, a fellow graduate student in the Molecular Pathogenesis \& Therapeutics (formerly Molecular Microbiology \& Immunology - Veterinary Pathobiology) program at the University of Missouri-Columbia.

Anna has always been interested in science-as a child she would ask her mother and father to "assign" her research projects so that she could have an excuse to read the encyclopedia. As a child, her favorite topics included rabbits and sharks. Although she is still enthralled with sharks, her scientific passion shifted to microbiology. As a student at Missouri Southern State University, she became fascinated with pathogenic bacteria, which led to her pursuit of a Microbiology graduate program. Upon entering graduate school, she learned that the disease resulting from bacterial infection had much less to do with the bacteria itself than the immune response to the pathogen, causing her to become enthralled with immunology—mostly innate immunology, obviously, because it is much more interesting than adaptive immunology (in her personal opinion and much to the chagrin of her husband, Cody, an adaptive immunologist).

Anna has always been passionate about changing the world and helping

others. With these two ideals in mind, she is pursuing a career in education. Her ultimate goal is to be a microbiology/immunology professor at a teaching 
university so that she can inspire in others the excitement that she felt as a student. She is also a passionate advocate for scientific communication and hopes to pursue projects that will allow her to break the barrier between science and public understanding. 